
Printed at the Mathematical Centre, 413 Kruislaan, Amsterdam.

The Mathematical Centre, founded the 11-th of February 1946, is a nonprofit institution aiming at the promotion of pure mathematics and its applications. It is sponsored by the Netherlands Government through the Netherlands Organization for the Advancement of Pure Research (Z.W.O.). 
MATHEMATICAL CENTRE TRACTS 129

\section{SIMPLICIAL \\ FIXED POINT \\ ALGORITHMS}

G. VAN DER LAAN

WITH THE COLLABORATION OF A.J.J. TALMAN

MATHEMATISCH CENTRUM AMSTERDAM 1980 


\section{ACKNOWLEDGEMENTS}

I am indebted to professor A.H.Q.M. Merkies, professor H.C. Tijms,

professor R. Saigal and professor M.J. Todd who read the manuscript and made a number of helpful comments.

This monograph is largely based on papers written in co-authorship with Dolf Talman. I am most grateful to him for the stimulating discussions and fine co-operation.

Finally thanks are due to Arie Oudshoorn for his assistance in writing the computer programs and to Gloria Wagenaar and Henk Kruijer for their excellent typing of the manuscript.

I thank the Mathematical Centre for the opportunity to publish this monograph in their series Mathematical Centre Tracts and all those at the Mathematical Centre who have contributed to its technical realization. 

CONTENTS

CHAPTER 1. INTRODUCTION 1

CHAPTER 2. PREIJIMINARIES 5

2.1. Introduction. 5

2.2 Brouwer's theorem. 5

2.3 Technique of triangulation. $\quad 8$

2.4 Sperner's lemma. 11

2.5 Kakutani's theorem. 14

2.6 Applications. 16

CHAPTER 3. TRIANGULATIONS AND MEASURES OF EFFICIENCY 21

3.1 Introduction. 21

3.2 Basic triangulations of $R^{n}$. 21

3.3. Measures for the efficiency of triangulations. 23

3.4 Triangulations of $\mathrm{s}^{\mathrm{n}}$ and $\mathrm{T}^{\mathrm{n}}$. 27

3.5 Replacement step. $\quad 29$

CHAPTER 4. ALGORITHMS FOR APPROXIMATING FIXED POINTS 31

4.1 Introduction. 31

4.2 Integer labelling and approximation. 32

4.3. Scarf's algorithm. $\quad 35$

4.4 Kuhn's algorithms. $\quad 36$

4.5 Algorithms to compute fixed points on $R_{+}^{n}$. 44

4.6 Sandwich method. $\quad 47$

4.7 Homotopy algorithm. $\quad$ - 49

4.8 Vector labelling and approximation. 53

4.9 A basic algorithm to compute a fixed point of a mapping. 55

4.10 Sandwich and homotopy methods on $R^{n}$. 57

4.11 Mappings from $\mathrm{S}^{\mathrm{n}}$ into $\mathrm{S}^{\mathrm{n}}$. 59

4.12 Concluding remarks. $\quad 61$ 
CHAPTER 5. VARIABLE DIMENSION RESTART ALGORITHM 63

5.1 Introduction.

5.2 Description of the steps of the algorithm. 64

5.3 Geometric interpretation. $\quad 68$

5.4 Convergence of the algorithm.

5.5 Interpretation of the algorithm in $s^{\mathrm{n}} \times[0,1]$. 75

5.6 Vector labelling. $\quad 79$

5.7 Convergence conditions for mappings on $\mathrm{T}^{\mathrm{n}}$. 85

5.8 Computational results. 91

CHAPTER 6. VARIABLE DIMENSION RESTART ALGORITHM ON R ${ }^{\mathrm{n}} 95$

6.1 Introduction. 95

6.2 The unit cube. 95

6.3 Convergence conditions on $R^{n}$. 101

6.4 Recent developments. 110

6.5 Computational results. 112

CHAPTER 7. THE ORIENTATION OF SIMPLICES $\quad 115$

7.1 Introduction. 115

7.2 Orientation. 116

7.3 The variable dimension restart algorithm. 122

7.4 A search to an odd number of completely labelled simplices. 130

CHAPTER 8. A CONTINUOUS DEFORMATION ALGORITHI WITH ARBITRARY

REFINEMENT FACTORS 139

8.1 Introduction. 139

8.2 Triangulation of $\mathrm{S}^{\mathrm{n}} \times[1, \infty)$. 140

$\begin{array}{ll}8.3 \text { The algorithm. } & 147\end{array}$

8.4 The replacement steps. 149

8.5 The application on $R^{n}$. 155

\begin{tabular}{lr}
8.6 & Some remarks and numerical results. \\
\hline
\end{tabular}

$\begin{array}{lr}\text { REFERENCES } & 163\end{array}$ 
CHAPTER 1

INTRODUCTION

Many problems in economics, nonlinear programming and other fields can be solved by using fixed point algorithms, which should be regarded as one of the major breakthroughs in computational methods for nonlinear problems. Already in 1910 the Dutch mathematician L.E.J. Brouwer proved that any continuous function $f$ from a compact convex set into itself has at least one fixed point, i.e. there exists at least one point $x^{*}$ in the set such that $f\left(x^{*}\right)=x^{*}$. The generalization of this theorem to upper semi-continuous point to set mappings is due to Von Neumann [1937] and Kakutani [.1941]. However, the originial proofs of these theorems were not concerned with computational methods. In spite of the simplification of the proofs during the subsequent years and the widespread use of the theorems as a mathematical tool in several fields it is only in the last fifteen years that fixed point theorems have been embedded in a computational setting.

The first method to compute a fixed point of a mapping from the unit simplex $S^{n}=\left\{x \in R_{+}^{n} \mid \Sigma_{i=1}^{n+1} x_{i}=1\right\}$ into itself was presented in the pioneering work of Scarf [.1967b], see also Scarf [1973]. He utilized an argument of Lemke and Howson [1964] and Lemke [1965] and the relationship of Sperner's lemma [1928] to the Brouwer fixed point theorem. Lemke and Howson provided a finite method for the computation of a Nash equilibrium point in a two-person nonzero-sum game using ideas from the pivot theory introduced by Dantzig [1951,1963] for the solution of linear programming problems. To prove the convergence of the method, Lemke did not use a monotonicity property but a combinatorial argument. To solve problems with a nonlinear character, Scarf replaced the sequence of linear programming pivot steps by the alternative construction of primitive sets, introduced by Scarf in an earlier paper r1967a.]. Scarf proved that the algorithm generates a path of adjacent primitive sets, and terminates within a finite number of iterations with a primitive set which 
yields a good approximation of a fixed point. Unfortunately, the procedure needs a high computer storage. A method to overcome this difficulty was discovered by Hansen [1963]. He chose the points of the primitive sets in a systematic way. However, then the pivot steps become identical to the pivot steps using a regular triangulation discovered by Freudenthal [1942] and made operational by Kuhn [1960]. Most of the recently developed fixed point algorithms are based on the concept of a triangulation. The first of them were already developed in 1968 and 1969 by Kuhn.

The algorithms mentioned above suffer from the disadvantage that the mesh of the triangulation is fixed and that they must start outside the region of interest. This means that when on a certain mesh an approximate fixed point is found the information obtained is of no use to find an approximation on a finer mesh. Fortunately, several algorithms followed, which were able to handle this drawback. A first method is due to Merrill [1971,1972] and was later rediscovered by Kuhn and Mackinnon [1975]. Closely related methods can be found in Lüthi [1975] and Fisher, Gould and Tolle [1977]. This algorithm, called the sandwich method, can start at an arbitrary point. This opens the opportunity to use the approximation obtained by applying the algorithm on a certain mesh as the starting point in a subsequent application with a finer mesh. A second method was independently and simultaneously discovered by Eaves [1972] for mappings on $S^{n}$ and generalized by Eaves and Saigal [1972] for mappings on $\mathrm{R}^{\mathrm{n}}$. In this method the mesh of the triangulation is automatically refined during the course of the algorithm. In both methods an extra dimension is used. They can be considered as methods which trace a path from the solution of an artificial mapping to the approximation of a solution of the original one. Algorithms without an extra dimension were developed by Tuy [1979] and by Garcia [1975] and Garcia and Gould $[1976,1979]$. However, as argued by Todd [1978b], the method of Tuy needs a lot of computation time whereas the algorithm of Garcia and Gould may fail to find an approximate fixed point.

Beside the development of more sophisticated algorithms many related issues were studied. Computational experiences showed that the computation time depends highly upon the underlying triangulation (see e.g. Saigal [1977a] and Todd [1978a]). Measures for the efficiency of a triangulation of $\mathrm{R}^{\mathrm{n}}$ were developed by Saigal, Solow and Holsey [1975] and Todd $[1976 b, 1978 a]$. However, for these measures it is not known how the optimal triangulation looks like. Van der Laan and Talman [1980a] suggested a new 
measure which enables the calculation of the optimal triangulation within a reasonable class. They also proposed a triangulation of the affine hull of the unit simplex whose measure is the same as that of the optimal triangulation of $R^{n}$. An other important subject is the convergence of fixed point algorithms on unbounded regions. Conditions that guarantee convergence were studied by Merrill [1971,1972], Gould and Tolle [1975], Saigal [1977c], Todd [1976a,1978b,1930], Saigal and Todd [1978], Reiser [1978a,b], Van der Laan and Talman [1980c] and Talman [1980]. To apply fixed point algorithms the grid points of a triangulation are labelled with an integer or vector label. The relation between convergence and labelling rules was studied by Fisher, Gould and Tolle [1977], Mackinnon [1977], Garcia and Gould [1976,1979] and Reiser [1978a]. Recently, Kojima [.1978a] and Todd [1978c,d] developed pivot methods which take advantage of the separability respectively the linearity of a function. Under certain conditions the convergence of a simplicial algorithm can be improved near a solution of a smooth mapping. Acceleration techniques which result in superlinear convergence were given by saigal [1977c] and Saigal and Todd [1978] for vector labelling (see also rolsey 「1974」). In these studies the approximate Jacobian (see Todd [1978e] and also Saigal [1979b]) plays an important role. An accelerated version of an algorithm using integer labelling was given by Reiser [1978a].

A development resulting in more insight into the simplicial fixed point algorithms is the theory of orientations and index. This theory was introduced by Shapley [1974] for bimatrix games and generalized by Lemke and Grotzinger [1976], Eaves and Scarf [1976] and Todd [1976c]. The path of simplices generated by fixed point algorithms was studied by Saigal [1976], Garcia and Gould [1978] and Saari and Saigal [1979]. Survey papers about fixed point algorithms and the related topics are those of Gould and Tolle [1974], Eaves [1976], Saigal [1977b] and Allgower and Georg [1980]. We refer the reader also to the monographs of Todd [1976a] and Lüthi [1976] and to the conference proceedings edited by Karamardian [1977] and Peitgen and valther [1979].

In this monograph new algorithms to compute a fixed point are presented. Also conditions to guarantee the convergence of the algorithms on unbounded regions are stated. Most of the work is based on the papers of Van der Laan and Talman $[1979 a, b, 1980 b, c]$. The monograph is organized as follows. In chapter 2 we give the most 
important fixed point theorems and some applications as the equilibrium price vector in economic models and the solution of an unconstrained optimization problem. Also the concepts of triangulation and piecewise linear approximation to a mapping with respect to a triangulation are given.

In chapter 3 triangulations of $R^{n}$ and $s^{n}$ are discussed together with measures for the efficiency of a triangulation.

Chapter 4 presents a survey of the algorithms of Scarf, Kuhn, Merrill and Eaves and Saigal. We show that the two algorithms of Kuhn are the two extreme cases of a class of algorithms. It will appear that the algorithms of Merrill and Eaves and Saigal supercede those of Scarf and Kuhn.

In chapter 5 a new algorithm to compute fixed points on $S^{\mathrm{n}}$ and its affine hull $T^{n}$ is introduced. The method is a restart algorithm and generates a path of simplices of variable dimension. In contrast to the algorithms of Merrill and Eaves and Saigal no extra dimension is needed.

In chapter 6 the application of the algorithm on the unit cube and on $R^{n}$ is discussed. Conditions to guarantee the convergence of the algorithm on $\mathrm{R}^{\mathrm{n}}$ are given.

In chapter 7 we define an orientation of a simplex. In view of the algorithm introduced in the chapters 5 and 6 we generalize this concept. Some preliminary results about the number of completely labelled simplices are also given. Also a method which gives an odd number of completely labelled simplices is discussed.

Chapter 8 presents another new algorithm. It is a method with an automatic refinement of the grid size. It differs from the algorithm of Eaves and Saigal in that it allows for any factor of refinement of the grid size. 
CHAPTER 2

PRELIMINARIES

\subsection{INTRODUCTION.}

Fixed point properties of functions or mappings are of fundamental importance in many problems e.g. the existence of a solution to a system of nonlinear equations or the existence of an equilibrium in an economy can be proved by using a fixed point theorem. The most famous fixed point theorem is due to Brouwer [1912]. We give a proof of this theorem using a combinatorial lemma of Sperner [1928]. We follow this approach since this lemma is closely related to arguments which play an important role in algorithms for computing fixed points. In particular the concept of a triangulation of a set is of importance.

In section 2 Brouwer's theorem is stated. Moreover, a lemma of Knaster, Kuratowski and Mazurkiewicz [1929] is given and it is proved that this lemma implies Brouwer's fixed point theorem. In section 3 we introduce the concept of a triangulation and some other definitions. In section 4 Sperner's lemma is given and Brouwer's theorem is proved whereas in section 5 the generalization of this theorem by Kakutani [1941] is discussed. Also the crucial concept of a piecewise linear approximation of a mapping is introduced here. In section 6 some applications of fixed point theorems are given.

\subsection{BROUWER'S THEOREM .}

Let $C$ be a nonempty set in the $n$-dimensional Euclidian space $R^{n}$, endowed with the standard norm $\|x\|=\left(\sum_{i=1}^{n} x_{i}^{2}\right)^{\frac{1}{2}}$. It is an important problem whether for any continuous function $f$ from $C$ into itself there exists at least one point $x^{*}$ in $C$ such that $f\left(x^{*}\right)=x^{*}, i$.e. whether such a function has at least one fixed point. Under the intuitively plausible conditions of convexity and compactness of $\mathrm{C}$ this statement was first proved by Brouwer. 
THEOREM 2.2.1. (BROUWER). Let $C$ be a compact and convex subset of $R^{n}$. Then any continuous function $\mathrm{f}: \mathrm{C} \rightarrow \mathrm{C}$ has at least one fixed point.

It is easy to see that the theorem is true for $n=1$. If $n>1$ the proof of the theorem is much more complicated. Without loss of generality we can restrict ourselves to continuous functions on the unit simplex.

DEFINITION 2.2.2. The $\mathrm{n}$-dimensional unit simplex $\mathrm{s}^{\mathrm{n}}$ is defined by

$$
S^{n}=\left\{x \in R^{n+1} \mid \sum_{i=1}^{n+1} x_{i}=1 \text { and } x_{i} \geq 0, i=1, \ldots, n+1\right\} ;
$$

for $i=1, \ldots, n+1$ we define the $i$-th boundary $s_{i}^{n}$ of $s^{n}$ by

$$
s_{i}^{n}=\left\{x \in s^{n} \mid x_{i}=0\right\}
$$

Observe that the vertices of $s^{n}$ are the $(n+1)$-dimensional unit vectors to be denoted by e(i), $i=1, \ldots, n+1$. In particular, $s^{2}$ is the equilateral triangle with the vertices $e(1), e(2)$ and $e(3)$. Following Todd [1976a] we prove Brouwer's theorem by using Sperner's lemma and the lemma of Knaster, Kuratowski and Mazurkiewicz [1929] (the $\mathrm{K}-\mathrm{K}-\mathrm{M}$ lemma).

LEMMA 2.2.3. (K-K-M). Let $\left\{\mathrm{C}_{1}, \ldots, \mathrm{C}_{\mathrm{n}+1}\right\}$ be a collection of closed subsets of $S^{\mathrm{n}}$ such that

$$
\begin{aligned}
& \text { a) } s^{n}=\underset{i=1}{U} C_{i} C_{i} \\
& \text { b) } \operatorname{n}_{i \in I}^{n} s_{i}^{n} \subseteq \underset{j \notin I}{U} C_{j} \text { for any } I \subset\{1, \ldots, n+1\} \text {. }
\end{aligned}
$$

$n+1$

Then $\bigcap_{i=1}^{n} C_{i}$ is nonempty.

The $\mathrm{K}-\mathrm{K}-\mathrm{M}$ lemma is illustrated for $\mathrm{n}=2$ in figure 2.2.1. 


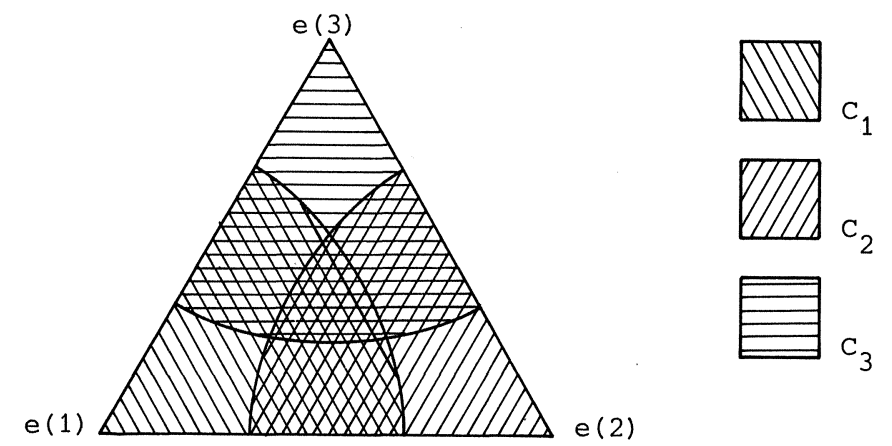

Figure 2.2.1. The K-K-M lemma.

We prove the lemma in section 4. Here we show that the theorem of Brouwer is implied by the lemma of $\mathrm{K}-\mathrm{K}-\mathrm{M}$.

LEMMA 2.2.4. The K-K-M lemma implies Brouwer's theorem.

PROOF. Define $c_{i}$ to be the set $\left\{x \in s^{n} \mid f_{i}(x) \leq x_{i}\right\}$ for all $i=1, \ldots, n+1$. Then it can easily be verified that the sets $C_{1}, \ldots, c_{n+1}$ satisfy the two conditions of the $K-K-M$ lemma. Therefore ${ }_{i=1}^{n+1} c_{i} \neq \emptyset$. Choose $x^{*} \in{ }_{i=1}^{n+1} c_{i}$. From the definition of $C_{i}$ we obtain that

$$
f_{i}\left(x^{*}\right) \leq x_{i}^{*} \quad i=1, \ldots, n+1
$$

Since $\Sigma_{i=1}^{n+1} f_{i}\left(x^{*}\right)=\Sigma_{i=1}^{n+1} x_{i}^{*}=1$ it follows that $f_{i}\left(x^{*}\right)=x_{i}^{*}, i=1, \ldots, n+1$. Hence $x^{*}$ is a fixed point of $f$.

To prove the $K-K-M$ lemma, we need the fundamental concept of a triangulation. This concept will be discussed in the next section. 


\subsection{TECHNIQUE OF TRIANGULATION.}

Before defining a triangulation of a set we introduce some basic concepts. Let $w^{i} \in R^{n}$ for $i=1, \ldots, t+1$. The $t+1$ points $w^{1}, \ldots, w^{t+1}$ are called affinely independent if $\Sigma_{i=1}^{t+1} \lambda_{i} w^{i}=0$ and $\Sigma_{i=1}^{t+1} \lambda_{i}=0$ imply $\lambda_{i}=0$ for $i=1, \ldots, t+1$. The convex hull of $t+1$ points $w^{1}, \ldots, w^{t+1}$ is the set

$$
\left\{x \in R^{n} \mid x=\sum_{i=1}^{t+1} \alpha_{i} w^{i} \text { with } \alpha \in S^{t}\right\}
$$

The convex hull of a set $D$, to be denoted by conv(D), is defined as the intersection of all convex sets containing $D$. The point $x=\sum_{i=1}^{t+1} \alpha_{i} w^{i}$ is called an affine combination of the points $w^{1}, \ldots, w^{t+1}$ if

$\Sigma_{i=1}^{t+1} \alpha_{i}=1$. The affine hull of a set $D$, to be denoted by aff(D), is the set of all finite affine combinations of points of $D$.

Now we give the following definitions.

DEFINITION 2.3.1. A t-dimensional simplex or $t$-simplex, to be denoted by $\sigma$, is the convex hull of $t+1$ affinely independent points $w^{1}, \ldots, w^{t+1}$ of $\mathrm{R}^{\mathrm{n}}$. We write $\sigma=\sigma\left(\mathrm{w}^{1}, \ldots, \mathrm{w}^{\mathrm{t}+1}\right)$. The points $\mathrm{w}^{1}, \ldots, \mathrm{w}^{\mathrm{t}+1}$ are called the vertices of $\sigma$.

DEFINITION 2.3.2. Let $\sigma$ be a $t$-simplex in $R^{n}$. Then a $k$-simplex $\tau(k \leq t)$ is a face of $\sigma$ if all the vertices of $\tau$ are vertices of $\sigma$. A $(t-1)$-face of $\sigma$ is called a facet of $\sigma$. The facet $\tau$ of $\sigma$ is said to be opposite to the vertex $w^{i}$ if $w^{i}$ is a vertex of $\sigma$ but is not a vertex of $\tau, i=1, \ldots, t+1$. Note that a $t$-simplex has $t+1$ facets and that the vertex opposite to a facet of $\sigma$ is uniquely determined.

DEFINITION 2.3.3. Two different simplices $\sigma_{1}$ and $\sigma_{2}$ are adjacent either if one of them is a facet of the other or if $\sigma_{1}$ and $\sigma_{2}$ share a common facet.

Let $\mathrm{C}$ be an m-dimensional convex subset of $\mathrm{R}^{\mathrm{n}}$. 
DEFINITION 2.3.4. A collection $\mathrm{G}$ of m-simplices is a triangulation of $\mathrm{C}$ if

a) C is the union of all simplices in $G$,

b) the intersection of two simplices in $G$ is either empty or a common face.

This definition excludes the cases drawn in figure 2.3.1. In the first case e.g. the intersection of $\sigma_{1}$ and $\sigma_{2}$ is neither empty nor a common face and in the second case $\sigma$ is not a simplex. Examples of a triangulation are shown in figure 2.3.2.
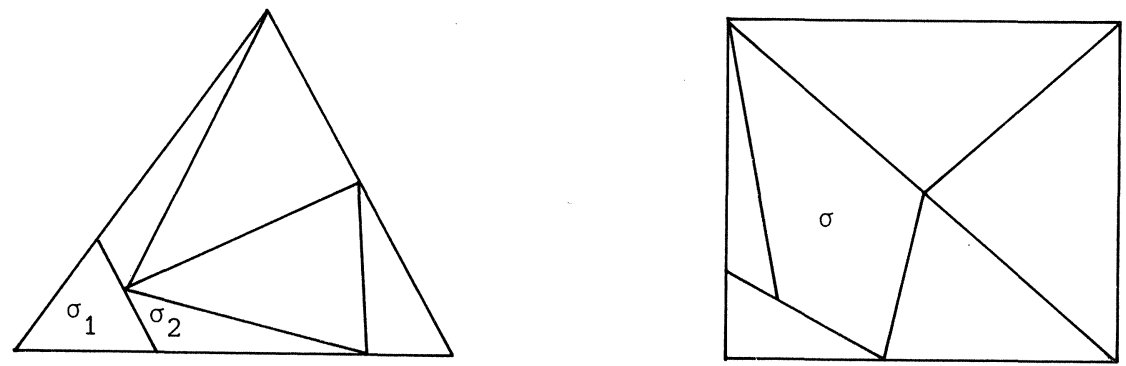

Figure 2.3.1. Examples which are not a triangulation.
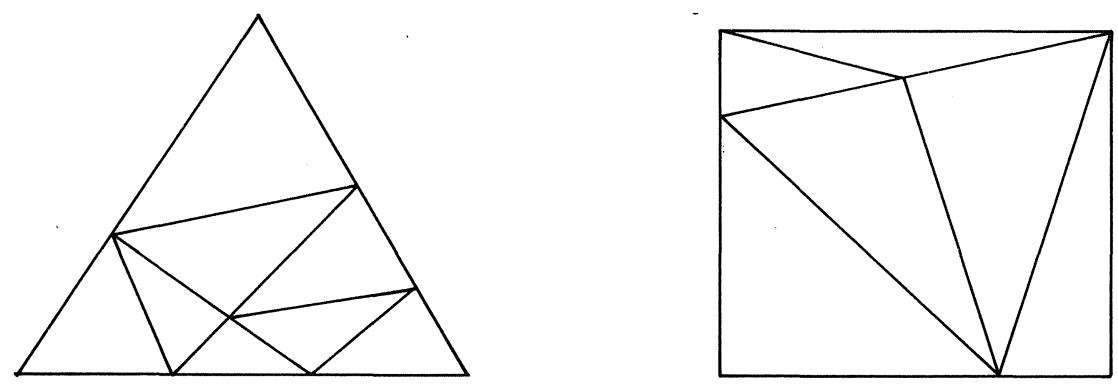

Figure 2.3.2. Examples of a triangulation. 
Some important properties of a triangulation are given in the following two theorems. Their proofs can be found in spanier [1966]. For any set $C \subset R^{n}$, denote by bd $C$ and int $C$ the boundary of $C$ respectively the interior of C, relatively to the affine hull of C, Cf. Berge [1966].

THEOREM 2.3.5. If $\tau$ is a facet of a simplex of a locally finite triangulation $G$ of the m-dimensional convex subset $C$ of $R^{n}$ then either $\tau$ belongs to bd $C$ and is a facet of just one simplex of $G$, or $\tau$ does not belong to bd $C$ and is a facet of exactly two simplices of $G$.

THEOREM 2.3.6 Let $\mathrm{D}$ be an (m-1)-dimensional subset in bd $\mathrm{C}$ such that $C \cap$ aff(D) is contained in D. Then $D$ is triangulated by the set of (m-1)simplices which are contained in $D$ and which are facets of simplices of the triangulation $\mathrm{G}$ of $\mathrm{C}$.

A triangulation $\mathrm{G}$ of $\mathrm{C}$ is locally finite is each $\mathrm{x} \in \mathrm{C}$ has a neighborhood meeting only a finite number of simplices of G. Although in chapter 6 a nonlocally finite triangulation will be described, in the remaining of this monograph we only deal with triangulations having the property of theorem 2.3.5.

Both theorems are illustrated by figure 2.3.3, where $\mathrm{C}$ is the 2-dimensional unit simplex. In this figure $\tau_{1}$ does not belong to bd $C$ and is a facet of the two simplices $\sigma_{1}$ and $\sigma_{2}$, whereas $\tau_{2}$ belongs to bd $C$ and is a facet of just one simplex. This simplex is $\sigma_{3}$. The triangulation of the convex hull of $e(2)$ and $e(3)$ consists of the three one-dimensional simplices $\tau_{2}, \tau_{3}$ and $\tau_{4}$.

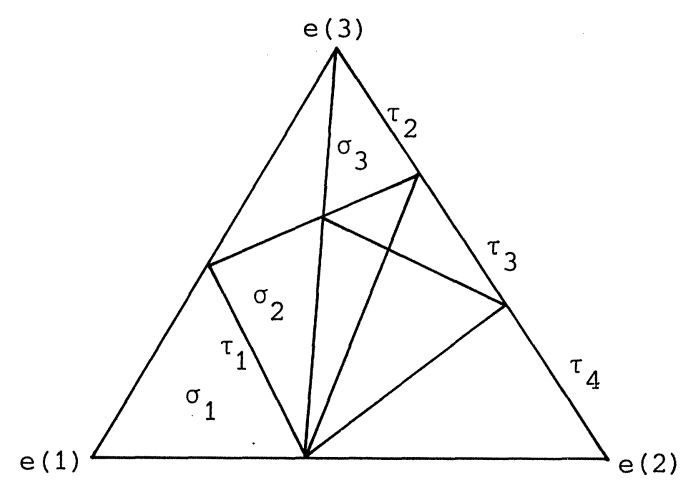

Figure 2.3.3. Illustration of the theorems 2.3 .5 and 2.3.6. 
We now define the mesh of a triangulation.

DEFINITION 2.3.7. Let $C$ be an m-dimensional convex subset of $R^{n}$ and let $G$ be a triangulation of $C$. The diameter of a simplex $\sigma$ of $G$, to be denoted by diam $\sigma$, is defined by

$$
\operatorname{diam} \sigma=\max _{x, y \in \sigma}\{|| x-y||\}
$$

The mesh of a triangulation $G$, to be denoted by mesh $G$, is defined by

$$
\operatorname{mesh} G=\sup _{\sigma \in G}\{\text { diam } \sigma\}
$$

Observe that the diameter of a simplex $\sigma$ with vertices $w^{1}, \ldots, w^{m+1}$ is equal to the maximum of the distance between two vertices, i.e.

$$
\operatorname{diam} \sigma\left(w^{1}, \ldots, w^{m+1}\right)=\max _{1 \leq i, j \leq m+1}\left\{|| w^{i}-w^{j} \|\right\}
$$

The following lemma is obvious.

LEMMA 2.3.8. Let $G_{1}$ be the set of all one-faces of the simplices of $G$. Then

$$
\text { mesh } G=\sup _{\tau \in G_{1}}\{\text { diam } \tau\}
$$

After this introduction of the concept of a triangulation of a set $C$, we are able to prove the $\mathrm{K}-\mathrm{K}-\mathrm{M}$ lemma by using a lemma of sperner.

\subsection{SPERNER'S LEMMA .}

Let $G$ be a triangulation of the m-dimensional convex subset $C$ of $R^{n}$ and assume that each vertex of the triangulation is labelled with one of the integers $1, \ldots, \mathrm{m}+1$, i.e. there is a labelling function $l$ from the set of vertices to the set $\{1, \ldots, m+1\}$. In the following the set $\{1, \ldots, n\}$ of 
integers will be denoted by $I_{n}$.

DEFINITION 2.4.1. An m-simplex of the triangulation $G$ is completely labelled if all its vertices are differently labelled, i.e. $\sigma\left(w^{1}, \ldots, w^{m+1}\right.$ ) is completely labelled if $\ell\left(w^{i}\right) \neq \ell\left(w^{j}\right)$ for all $i \neq j, j=1, \ldots, m+1$. Otherwise stated we have that if for any $j \in I_{m+1}$ there exists an index $i_{j} \in I_{m+1}$ such that $\ell\left(w^{i} j\right)=j$, then $\sigma\left(w^{1}, \ldots, w^{m+1}\right)$ is completely labelled.

The following lemma due to Sperner [1928] gives a condition for the existence of a completely labelled simplex of a triangulation of the unit simplex $s^{\mathrm{n}}$.

LEMMA 2.4.2. (SPERNER). Let $G$ be some triangulation of $\mathrm{s}^{\mathrm{n}}$. Assume that each vertex of its n-simplices is labelled with one of the integers of the set $I_{n+1}$, such that no vertex in $s_{i}^{n}$ has label $i$ for all $i=1, \ldots, n+1$. Then there exists at least one completely labelled n-simplex.

The lemma is obvious for $\mathrm{n}=1$ as is illustrated in figure 2.4.1.

The one-simplices $\sigma_{1}, \sigma_{2}$ and $\sigma_{3}$ are completely labelled. For $\mathrm{n}=2$ the lemma is illustrated in figure 2.4.2.
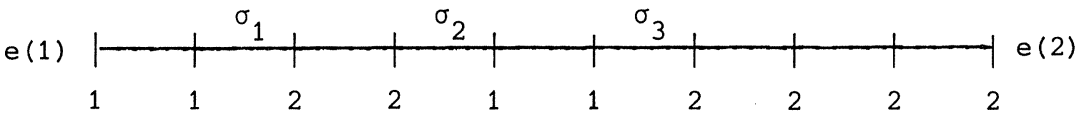

Figure 2.4.1. Illustration of Sperner's lemma for $n=1$.

A proof of Sperner's lemma by induction on $\mathrm{n}$ can be found in Todd [1976a]. He proves a strong form of the lemma, which says that there is an odd number of completely labelled simplices. We do not give the proof, since the lemma follows as a corollary in chapter 4. 


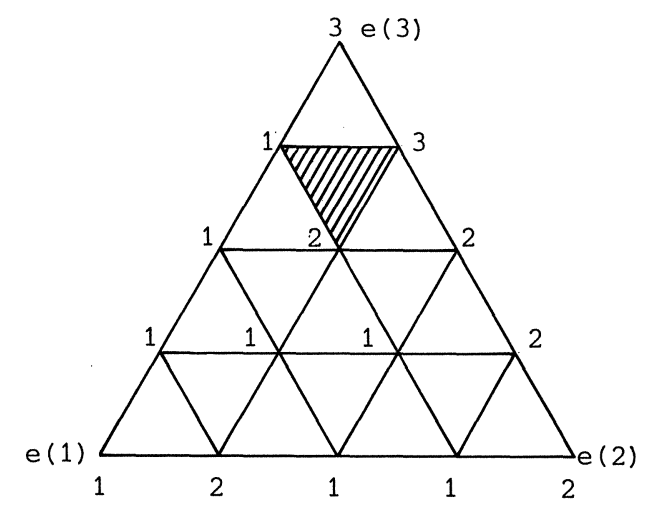

Figuur 2.4.2. Illustration of Sperner's lemma for $\mathrm{n}=2$.

The following lemma completes the proof of Brouwer's theorem.

LEMMA 2.4.3. Sperner's lemma implies the lemma of K-K-M.

PROOF. Let the collection $\left\{\mathrm{c}_{1}, \ldots, \mathrm{c}_{\mathrm{n}+1}\right\}$ of closed subsets of $\mathrm{s}^{\mathrm{n}}$ be defined as in lemma 2.2.4. Furthermore, $\operatorname{let}\left\{G_{k}, k=1,2, \ldots\right\}$ be some sequence of triangulations of $s^{n}$ such that $\varepsilon_{k}=$ mesh $G_{k} \rightarrow 0$ if $k \rightarrow \infty$. Each point $y$ in $s^{\mathrm{n}}$ receives an integer label $\ell(y)$ defined by

$$
\ell(y)=i \text {, where } i=\min _{j}\left\{j \mid y \in C_{j} \text { and } y_{j}>0\right\}
$$

Note that, by the definition of $C_{j}$, there exists always such an index $i$. Since this labelling satisfies the condition of sperner's lemma, there is at least one completely labelled simplex $\sigma_{k}$ in $G_{k}$. Let $y^{i}(k)$ be the vertex of $\sigma_{k}$ with label $i, i=1, \ldots, n+1$. Then for some subsequence $\left\{k_{j}, j=1,2, \ldots\right\}$ of integers with $k_{j} \rightarrow \infty$ if $j \rightarrow \infty$ we have that the sequence $\left\{y^{1}\left(k_{j}\right), j=1,2, \ldots\right\}$ converges to a point $x^{*}$ in $s^{n}$. Since $\varepsilon_{k} \rightarrow 0$ if $k \rightarrow \infty$ it follows that $\lim _{j \rightarrow \infty} y^{i}\left(k_{j}\right)=x^{*}$ for all $i=1, \ldots, n+1$. Hence $x^{*} \in C_{i}$, $i=1, \ldots, n+1$ which proves that $\prod_{i=1}^{n} c_{i} \neq \varnothing$. 
In the context of Brouwer's theorem this lemma means that, roughly speaking, any sequence of "shrinking" completely labelled simplices has a subsequence converging to a fixed point.

\subsection{KAKUTANI'S THEOREM.}

In many practical problems we need a fixed point theorem more general than the theorem of Brouwer. The theorem of Kakutani generalizes Brouwer's theorem to point-to-set mappings (cf. Berge [1966]). For any $\alpha>0$ let $\mathrm{B}^{\mathrm{n}}(\mathrm{x}, \alpha)$ be defined as the set $\left\{y \in \mathrm{R}^{\mathrm{n}}|||\mathrm{y}-\mathrm{x}| \mid \leq \alpha\right\}$.

DEFINITION 2.5.1. Let $\mathrm{C}$ be a subset of $\mathrm{R}^{\mathrm{n}}$ and let $\phi$ be a point-to-set mapping from $C$ to the set of all subsets of $R^{m}$. Then $\phi$ is upper-semicontinuous if

a) for all $\mathrm{x} \in \mathrm{C}, \phi(\mathrm{x})$ is compact,

b) for all $\mathrm{x} \in \mathrm{C}$ and for all $\varepsilon>0$ there is a $\delta>0$ such that for any $z \in B^{n}(x, \delta) \cap C$ holds $\phi(z) \in B^{m}(w, \varepsilon)$ for some $\mathrm{w} \in \phi(\mathrm{x})$.

An interesting property of upper-semicontinuous (u.s.c.) mappings is stated in the following corollary (cf. Todd [1976a]).

COROLLARY 2.5.2. Let $\phi$ be an U.s.c. mapping from $C$ to the set of subsets of $R^{m}$. If $\left\{x^{k}\right\}$ is a sequence of points in $C$ with limit $x^{*}$ and if $\left\{y^{k}\right\}$ is a sequence of points in $\mathrm{R}^{\mathrm{n}}$ with $\mathrm{y}^{\mathrm{k}} \epsilon \phi\left(\mathrm{x}^{\mathrm{k}}\right)$ for all $\mathrm{k}$ and with limitpoint $\mathrm{y}^{*}$, then $\mathrm{y}^{*} \in \phi\left(\mathrm{x}^{*}\right)$.

The following theorem gives a sufficient condition which guarantees that an u.s.c. mapping has a fixed point, i.e. a point $x^{*}$ such that $x^{*} \epsilon \phi\left(x^{*}\right)$.

THEOREM 2.5.3. (KAKUTANI). Let $\mathrm{C}$ be a compact, convex, nonempty m-dimensional subset of $\mathrm{R}^{\mathrm{n}}$ and let $\phi$ be an u.s.c. mapping from $\mathrm{C}$ to the set of nonempty convex subsets of $C$. Then there is a point $x^{*} \epsilon C$ such that $x^{*} \epsilon \phi\left(x^{*}\right)$. 
To prove this theorem we use the concept of a piecewise linear approximation to a mapping.

DEFINITION 2.5.4. Let $G$ be a triangulation of a nonempty, convex m-dimensional subset $C$ of $R^{n}$ and $\phi$ a nonempty mapping from $C$ to the set of subsets of $R^{p}$. For any vertex $w$ of $G$ let $f(w)$ be some arbitrarily chosen value of $\phi(w)$. Furthermore, let for a point $x \in C, w^{1}, \ldots, w^{t+1}$ be the vertices of the t-simplex such that $\mathrm{x}$ is contained in the interior of the simplex. Then there is a unique positive vector $\lambda=\left(\lambda_{1}, \lambda_{2}, \ldots \lambda_{t+1}\right)$ such that $\sum_{i=1}^{t+1} \lambda_{i}=1$ and $x=\sum_{i=1}^{t+1} \lambda_{i} w^{i}$. Then a piecewise linear approximation to the mapping $\phi$ with respect to $G$ is the function $f: C \rightarrow R^{P}$ defined by

$$
f(x)=\sum_{i=1}^{t+1} \lambda_{i} f\left(w^{i}\right) .
$$

COROLLARY 2.5.5. The piecewise linear approximation $f$ is continuous.

PROOF OF THEOREM 2.5.3. We follow the proof of Eaves [1971]. Let $C^{\prime}$ be an $\mathrm{m}$-simplex containing $\mathrm{C}$ and let $\mathrm{c}$ be an interior point of $\mathrm{C}$. Define the mapping $\bar{\phi}$ from $C^{\prime}$ to the set of nonempty, convex, compact subsets of $C^{\prime}$ by

$$
\begin{aligned}
\bar{\phi}(\mathrm{x}) & =\phi(\mathrm{x}) & & \text { if } x \in \text { int } C \\
& =\operatorname{conv}(\phi(x) \cup\{c\}) & & \text { if } x \in \text { bd } C \\
& =\{c\} & & \text { if } x \in C^{\prime} \backslash C .
\end{aligned}
$$

It is easy to see that $\bar{\phi}$ is upper-semicontinuous on $C^{\prime}$. Moreover, it is clear that $\bar{\phi}(\mathrm{x})$ is nonempty and convex for all $\mathrm{x} \in \mathrm{C}^{\prime}$. Suppose that $\mathrm{x}^{*}$ is a fixed point of $\bar{\phi}$. It is obvious that $x^{*} \epsilon C$. If $x^{*} \epsilon$ int $C$, then $\mathrm{x}^{*} \epsilon \phi\left(\mathrm{x}^{*}\right)$ and $\mathrm{x}^{*}$ is a fixed point of $\phi$. If $\mathrm{x}^{*} \epsilon$ bd $\mathrm{C}$, then for some $y \in \phi\left(x^{*}\right), x^{*}=\lambda c+(1-\lambda) y$ for some $\lambda, 0 \leq \lambda \leq 1$. Since $c \epsilon$ int $c$, we have that $\lambda>0$ implies that $\mathrm{x}^{*} \epsilon$ int $\mathrm{C}$ which contradicts $\mathrm{x}^{*} \epsilon$ bd $\mathrm{C}$. Hence, 
$\lambda=0$ and $x^{*}=y \in \phi\left(x^{*}\right)$. Therefore $x^{*}$ is a fixed point of $\phi$ if $x^{*}$ is a fixed point of $\bar{\phi}$. To prove that $\bar{\phi}$ has indeed a fixed point on $C^{\prime}$, let $\left\{G_{k}, k=1,2, \ldots\right\}$ be a sequence of triangulations of $C^{\prime}$ with $\varepsilon_{\mathrm{k}}=\operatorname{mesh} \mathrm{G}_{\mathrm{k}} \rightarrow 0$ if $\mathrm{k} \rightarrow \infty$, and let $\mathrm{f}^{\mathrm{k}}$ be a piecewise linear approximation to $\bar{\phi}$ with respect to the triangulation $G_{k}$. Bij Brouwer's theorem $f^{k}$ has a fixed point, say $\mathrm{x}^{\mathrm{k}}$. Moreover, by definition 2.5 .4 , there is a simplex of $G_{k}$ with vertices $w^{1}(k), \ldots, w^{m+1}(k)$ containing $x^{k}$ such that for unique nonnegative numbers $\lambda_{1}^{\mathrm{k}}, \ldots, \lambda_{\mathrm{m}+1}^{\mathrm{k}}$ with $\lambda^{\mathrm{k}}=\left(\lambda_{1}^{\mathrm{k}}, \ldots, \lambda_{\mathrm{m}+1}^{\mathrm{k}}\right)^{\top} \in \mathrm{s}^{\mathrm{m}}$ holds

$$
x^{k}=f^{k}\left(x^{k}\right)=\sum_{i=1}^{m+1} \lambda_{i}^{k} f^{k}\left(w^{i}(k)\right) .
$$

Since $C^{\prime}$ is compact, there is a subsequence $k_{j}, j=1,2, \ldots$ such that $x^{k_{j}} \rightarrow x^{*}, \lambda^{k_{j}} \rightarrow \lambda^{*}$ and $f^{k_{j}}\left(w^{i}\left(k_{j}\right)\right) \rightarrow \tilde{f}^{i}, i=1, \ldots, m+1$, if $j \rightarrow \infty$. Since $\varepsilon_{k} \rightarrow 0, w^{i}\left(k_{j}\right)$ converges to $x^{*}$ for all $i$. Because of the upper-semicontinuity of $\bar{\phi}, \tilde{f}^{i} \in \bar{\phi}\left(x^{*}\right)$, for all $i$. Taking limits in the equation above, we obtain that $x^{*}=\Sigma_{i=1}^{m+1} \lambda_{i}^{*} \tilde{f}^{i}$ with $\lambda^{*} \in \mathrm{s}^{\mathrm{m}}$, while $\tilde{f}^{i} \in \bar{\phi}\left(\mathrm{x}^{*}\right)$ for all i. Therefore, since $\bar{\phi}\left(x^{*}\right)$ is convex, $x^{*} \in \bar{\phi}\left(x^{*}\right)$, which completes the proof.

Note that the property of corollary 2.5.2 is sufficient to prove the theorem. Algorithms to compute "Kakutani fixed points" will be based on the technique of piecewise linear approximation to the mapping with respect to a sequence of triangulations with mesh going to zero.

\subsection{APPLICATIONS.}

In this section we give some examples of Brouwer's theorem and the theorem of Kakutani. With respect to Brouwer's theorem we discuss the problem of the existence of an equilibrium in an exchange economy. As an application of Kakutani's theorem we give the unconstrained optimization problem.

EXAMPLE 2.6.1. (Exchange economy).

Let us consider an economy without production in which $n+1$ commodities are exchanged. We assume that there are $\mathrm{m}$ consumers each having a utility function $u^{i}(x), i=1, \ldots, m$, where $x=\left(x_{1}, \ldots, x_{n+1}\right)$ denotes a vector of commodities. This function is defined from $R_{+}^{n+1}$ to $R$ and reveals the utility of the i-th consumer. Furthermore, we assume that each consurner has a 
vector of initial endowments $e^{i}=\left(e_{1}^{i}, \ldots, e_{n+1}^{i}\right)$. Given a price vector $p=$ $\left(p_{1}, \ldots, D_{n+1}\right)^{\top} \in R_{+}^{n+1} \backslash\{0\} \quad$ each consumer $i$ maximizes his utility under the budget constraint $p^{\top} x \leq p^{\top} e^{i}$. Let $x^{i}(p)$ be the demand vector for consumer $i$ which maximizes his utility given the price vector $p$. Clearly, $x^{i}(p)$ is homogeneous of degree zero in $\mathrm{p}$. Therefore we can normalize the price vector $p$ such that $p \in s^{n}$. So $x^{j}(p)$ is a function from $s^{n}$ to $R_{+}^{n+1}$ assuming that $x^{i}(p)$ exists and that $x^{i}(p)$ is unique for all $p$. Furthermore, we assume that $x^{i}(p)$ is continuous in $p$, for all i. The economic consequences of these assumptions are discussed by many authors, e.g. Arrow and Hahn [1971]. Finally, if $u^{i}(x)$ is a monotonic increasing function of $x$, each consumer spends all his income, i.e. $p^{\top} x^{i}(p)=p^{\top} e^{i}, \quad i=1, \ldots, m$. Then we have

$$
\underline{p}^{\top} \sum_{i=1}^{m}\left(x^{i}(p)-e^{i}\right)=0
$$

which formula is known as Walras' law. Since $x^{i}(p)$ is continuous for all $i$, the excess demand function $g$ from $S^{n}$ to $R^{n+1}$ defined by

$g(p)=\Sigma_{i=1}^{m}\left(x^{i}(p)-e^{i}\right)$ is also continuous in $p$. The economy is said to be in equilibrium if there exists a price vector $p^{*}$ in $s^{n}$ such that $g\left(p^{*}\right) \leq 0$. By Walras' law it is clear that in equilibrium $p_{j}^{*}=0$ if $g_{j}\left(p^{*}\right)<0$, $j=1, \ldots, m+1$.

Using Brouwer's fixed point theorem we prove that an equilibrium exists under the assumptions stated above. We define the function $f$ from $s^{n}$ into itself by

$$
f_{j}(p)=\left[p_{j}+\max \left\{0, g_{j}(p)\right\}\right] / c(p) \quad j=1, \ldots, n+1
$$

where $c(p)=1+\Sigma_{j=1}^{n+1} \max \left\{0, g_{j}(p)\right\}$. Clearly, f satisfies the conditions of Brouwer's theorem. Therefore, there exists a price $p^{*}$ such that $\underline{p}^{*}=f\left(p^{*}\right)$. We show that $p$ is a fixed point of the function $f$ if and only if $p$ is an equilibrium price vector. If. $\underline{p}^{*}$ is a fixed point of $f$ then

$$
p_{j}^{*}=\left[\underline{p}_{j}^{*}+\max \left\{0, g_{j}\left(\underline{p}^{*}\right)\right\}\right] / c\left(p^{*}\right) \quad j=1, \ldots, n+1 .
$$

Assume $g_{j}\left(p^{*}\right)>0$ for some $j$. Then $c\left(p^{*}\right)>1$ and hence $g_{k}\left(p^{*}\right)>0$ if $p_{k}^{*}>0$. This implies that $\sum_{j=1}^{n+1} \underline{p}_{j}^{*} g_{j}\left(p^{*}\right)>0$ which contradicts Talras' law. Consequently, $g_{j}\left(p^{*}\right) \leq 0 j=1, \ldots, n+1$, which proves that $p^{*}$ is an equilibrium price vector. Conversely, if $p^{*}$ is such a vector then 
$\max \left\{0, g_{j}\left(\underline{p}^{*}\right)\right\}=0, j=1, \ldots, n+1$ and hence $f\left(\underline{p}^{*}\right)=p^{*}$.

The function $f$ defined above is not particularly suitable for use in algorithms for computing fixed points, since by considering the maximum over zero and the excess demand in stead of the excess demand, information is lost about the structure of the function. In a later chapter the computation of an equilibrium price vector will be based on the excess demand.

For many economic models the existence of an equilibrium was proved, e.g. Debreu [1959], Drèze [1975], Greenberg [1977], Van der Laan [19807. In general Brouwer's theorem is not sufficient. For instance in an economy with linear production activities the excess demand is an uppersemicontinuous point-to-set mapping and the existence proof and computation of an equilibrium will be based on Kakutani's theorem (see e.g. Scarf [1973] and Todd $[1976 \mathrm{a}, 1977 \mathrm{~b}])$. About computational results for economic models obtained by using fixed point algorithms we refer the reader also to Shoven and Whalley [1973], Whalley [1975], Mackinnon [1976], Richter [1978] and Reif [1978].

Before we discuss the example of unconstrained optimization we give a useful extension of Kakutani's theorem. The next lemma is due to Merrill [1971,1972] (see also Todd [1976a] and Allgower and Georg [1980]).

LEMMA 2.6.2. (Merrill's condition).

Let $\phi$ be an upper-semicontinuous mapping from $R^{n}$ to the collection of nonempty, convex subsets of $R^{n}$. Assume that there exist $w \in R^{n}, \mu>0$ and $\delta>0$ such that for all $x \notin B^{n}(w, \mu), f(x) \in \phi(x)$ and $z \in B^{n}(x, \delta)$ holds

$$
(f(x)-x)^{\top}(w-z)>0 .
$$

Then the mapping $\phi$ has a fixed point in $B^{n}(w, \mu)$.

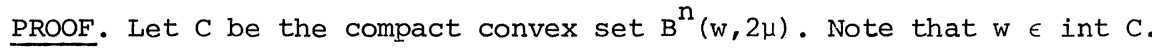
Define the mapping $\phi^{\prime}(\mathrm{x})$ by

$$
\begin{aligned}
\phi^{\prime}(x) & =\phi(x) & & \text { if } x \in \text { int } C \\
& =\operatorname{conv}(\phi(x) \cup\{w\}) & & \text { if } x \in \text { bd } C .
\end{aligned}
$$


Then $\phi^{\prime}(x)$ is an u.s.c. mapping from $C$ to the collection of nonempty convex subsets of $R^{n}$ such that $w \in \phi^{\prime}(x)$ for all $x \in$ bd $C$. Following Eaves [1971] we show that $\phi^{\prime}$ has a fixed point. Clearly, since $\phi^{\prime}$ is u.s.c. $\phi^{\prime}(C)$ is compact and hence the set $C^{\prime}$ defined by

$$
C^{\prime}=\operatorname{conv}\left(C \cup \phi^{\prime}(\mathrm{C})\right)
$$

is compact. Define the u.s.c. mapping $\bar{\phi}^{\prime}$ from $C^{\prime}$ into itself by

$$
\begin{aligned}
\bar{\phi}^{\prime}(x) & =\phi^{\prime}(x) & & \text { if } x \in C \\
& =\{w\} & & \text { if } x \in C^{\prime} \backslash C .
\end{aligned}
$$

Since $\bar{\phi}$ ' satisfies the conditions of Kakutani's theorem there exists a fixed point $x^{*} \in C^{\prime}$ of $\bar{\phi}^{\prime}$ and analogous as in the proof of theorem 2.5.3, it follows that $x^{*} \in C$ and that $x^{*}$ is a fixed point of $\phi^{\prime}\left(x^{*}\right)$. If $x^{*} \in B^{n}(w, \mu)$, then $x^{*} \epsilon$ int $C$ and hence $x^{*} \in \phi\left(x^{*}\right)$.

Therefore we prove that $x^{*} \in B^{n}(w, \mu)$. Suppose to the contrary that $x^{*} \notin B^{n}(w, \mu)$. Since $x^{*} \in \phi^{\prime}\left(x^{*}\right) \subset \operatorname{conv}\left(\phi\left(x^{*}\right) \cup\{w\}\right)$, for some $\lambda, \lambda \in[0,1]$, and some $f\left(x^{*}\right) \in \phi\left(x^{*}\right)$ we have that

$$
x^{*}=\lambda f\left(x^{*}\right)+(1-\lambda) w
$$

so that

$$
\lambda\left(f\left(x^{*}\right)-x^{*}\right)+(1-\lambda)\left(w-x^{*}\right)=0 .
$$

However, $\left(w-x^{*}\right)^{\top}\left(w-x^{*}\right)>0$ while by the condition of the theorem $\left(f\left(x^{*}\right)-x^{*}\right)^{\top}\left(w-x^{*}\right)>0$. So a contradiction is obtained which proves that $x^{*} \in B^{n}(w, \mu)$.

Lemma 2.6.2. is used in the following example.

EXAMPLE 2.6.3. (Unconstrained optimization problem). Let $f: R^{n} \rightarrow R$ be a convex and finite function. We want to compute a point $x$ such that $f(x) \leq f(z)$ for all $z \in R^{n}$. Let $d f(x)$ be the set of all subgradients of $f$ at $x$, i.e.

$$
d f(x)=\left\{y \in R^{n} \mid f(z) \geq f(x)+y^{\top}(z-x) \text { for all } z \in R^{n}\right\}
$$


Since $f$ is convex, the mapping df from $R^{n}$ to the set of nonempty, convex subsets of $R^{n}$ is u.s.c. (Rockafellar [1970]). Define the u.s.c. mapping $\phi$ by $\phi(x)=\{x\}-d f(x), x \in R^{n}$.

Assume there exists an $\alpha$ such that the set $F(\alpha)=\{y \mid f(y) \leq \alpha\}$ is nonempty and bounded. Then $F(\beta)$ is bounded for all real $\beta$ (Rockafeller [1970]). For arbitrarily chosen $w \in R^{n}$ and $\delta>0$, let $\beta=\max \left\{f(x) \mid x \in B^{n}(w, \delta)\right\}$. Then $F(\beta)$ is bounded so we can choose an $\mu>0$ such that $F(\beta) \subset B^{n}(w, \mu)$. Furthermore, for any $x \notin B^{n}(w, \mu), g(x) \in \phi(x)$ and $z \in B^{n}(x, \delta)$ we have that $x-g(x) \in d f(x)$ and hence

$$
(g(x)-x)^{\top}(w-z)=(g(x)-x)^{\top}\{(w-z+x)-x\} \geq-f(w-z+x)+f(x)
$$

By $x \notin B^{n}(w, \mu)$ we have $f(x)>\beta$ and by $w-z+x \in B^{n}(w, \delta)$ we have $-f(w-z+x)>-\beta$. Therefore $(g(x)-x)^{\top}(w-z)>0$, which implies that $\phi(x)$ satisfies Merrill's condition. So there exists a point $x^{*}$ with $x^{*} \epsilon \phi\left(x^{*}\right)$. Clearly $0 \in d f\left(x^{*}\right)$ and hence $f\left(x^{*}\right) \leq f(z)$ for all $z \in R^{n}$.

As an illustration of this example, we want to minimize $f(x)=$ $x_{1}^{2}+2 x_{2}^{2}-x_{1} x_{2}+x_{1}+x_{2}$. Then $d f(x)=\left(2 x_{1}-x_{2}+1,4 x_{2}-x_{1}+1\right)^{\top}$ and the function $\phi(x)=\left(-x_{1}+x_{2}-1, x_{1}-3 x_{2}-1\right)^{\top}$ has $(-5 / 7,-3 / 7)^{\top}$ as a fixed point. Clearly this point minimizes $f(x)$. For other applications of fixed point theorems see e.g. Todd [1976a], Saigal [1977b,1979a], Allgower and Georg [1980] and Talman [1980]. 
TRIANGULATIONS AND MEASURES OF EFFICIENCY

\subsection{INTRODUCTION.}

In this chapter we discuss special triangulations and measures for the efficiency of triangulations. Computational experience has shown that the efficiency of fixed point algorithms is very sensitive to the underlying triangulation (see e.g. Saigal [1977a] and Todd [1978a]) . Basic triangulations of $\mathrm{R}^{\mathrm{n}}$ used in fixed point algorithms are the so called $\mathrm{K}$ and $\mathrm{H}$ triangulations which are defined in section 2. Measures for the efficiency of a triangulation of $\mathrm{R}^{\mathrm{n}}$ were given by saigal, Solow and Wolsey [1975], Todd [1976b, 1978a], Van der Laan and Talman [1980a] and Talman [1980]. Whereas earlier measures do not allow the calculation of the optimal triangulation, for their measure Van der Laan and Talman succeeded in calculating an optimal triangulation within a reasonable subclass of triangulations. The measures and the optimal triangulation for the measure of Van der Laan and Talman [1980a] are given in section 3. Section 4 gives triangulations of the unit simplex and its affine hull including one which is comparable with the optimal triangulation of $R^{n}$. Finally in section 5 the replacement step between two full-dimensional adjacent simplices is given.

\subsection{BASIC TRIANGULATIONS OF $R^{n}$.}

Since the accuracy of an approximation of a fixed point will depend on the mesh of the triangulation we need triangulations without long, skinny simplices. In other words "regular" triangulations are preferred in fixed point algorithms (see Saigal [1977a, 1979b]). The first regular triangulation was constructed by Freudenthal [1942] and rediscovered by Tucker in 1945 (see Lefschetz [1949, page 140]). This triangulation is called the $\mathrm{K}$ triangulation and is based on the "standard" subdivision of the unit cube (Kuhn [1960]). 
DEFINITION 3.2.1. Let $\mathrm{z}$ be an arbitrarily chosen point in $\mathrm{R}^{\mathrm{n}}$. Then the $\mathrm{K}$ triangulation with grid size $\delta>0$ is the collection of al.l simplices $\sigma\left(y^{1}, \pi\right)$ with vertices $y^{1}, \ldots, y^{n+1}$ such that

a) each component of $y^{1}-z$ is a multiple of $\delta$

b) $\pi=\left(\pi_{1}, \ldots, \pi_{n}\right)$ is a permutation of the elements of the set $I_{n}$

c) $y^{i+1}=y^{i}+\delta e\left(\pi_{i}\right) \quad i=1, \ldots, n$.

In general $\mathrm{z}$ will be chosen as the zero vector. Observe that for $\delta=1$ the collection of simplices $\left\{\sigma(\underline{0}, \pi) \mid \pi\right.$ is a permutation of the elements of $\left.T_{n}\right\}$ triangulates the unit cube $c=\left\{x \in R^{n} \mid 0 \leq x_{i} \leq 1 \quad i=1, \ldots, n\right\}$. From the definition it follows that the diameter of each simplex is ecrual to $\delta\left\|\Sigma_{i=1}^{n} e(i)\right\|=\delta \sqrt{ }$. So, the mesh of the $k$ triangulation with grid size $\delta$ is equal to mesh $\mathrm{K}_{\delta}=\delta \sqrt{ } \mathrm{n}$. We denote the $\mathrm{k}$ triangulation with grid size $\delta$ by $\mathrm{K}_{\delta}$ and write $\mathrm{K}_{\delta}=\mathrm{K}$ when $\delta=1$.

A triangulation closely related to the $\mathrm{K}$ triangulation was used by MerrijI [1972] and Faves and Saigal [1972]. It is called the $\mathrm{H}$ triangulation. For $\mathrm{z}=0$ this triangulation is built up by simplices $\sigma\left(\mathrm{y}^{1}, \pi\right)$ such that the components of $y^{1}$ are again multiples of $\delta$ and $y^{i+1}=y^{i}+\delta \tilde{q}\left(\pi_{i}\right), i=1, \ldots, n$, where $\tilde{q}(j)$ is the $j$-th column of the $n \times n$ matrix $\tilde{Q}$ defined by

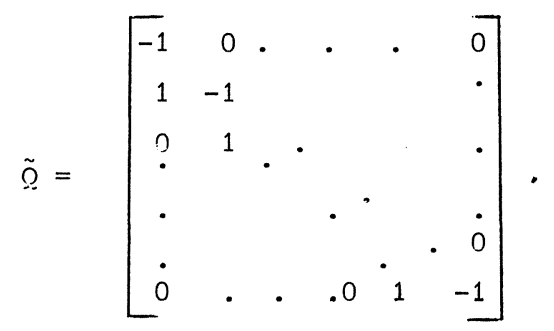

of course, any arbitrarily chosen point $z$ can be made a grid point by a shift of the grid, such that the components of $y^{1}-z$ become integer multiples of $\delta$. Clearly for the $H$ triangulation with grid size $\delta$, denoted by $H_{\delta}(H$ if $\delta=1)$, the mesh is equal to $\delta \max _{S \subset I_{n}}\left\|\sum_{j \in S} \tilde{q}(j)\right\|=\delta \sqrt{ }$.

More general, for any nonsingular matrix $A$, we can define the triangulation $\mathrm{AK}_{\delta}$ with grid size $\delta$ as follows. 
DEFINITION 3.2.2. Let $\mathrm{A}$ be a nonsingular $\mathrm{n} \times \mathrm{n}$ matrix. Then the triangulation $A K_{\delta}$ is the collection of all simplices $\sigma\left(A y^{1}, \ldots, A y^{n+1}\right)$ such that $\sigma\left(y^{1}, \ldots, y^{n+1}\right)$ is a simplex of $k_{\delta}$.

Observe that for $z=0$ the triangulation $A K$ is the collection of simplices $\sigma\left(\mathrm{y}^{1}, \pi\right)$ such that all components of $\mathrm{A}^{-1} \mathrm{y}^{1}$ are integers and $y^{i+1}=y^{i}+a\left(\pi_{i}\right) i=1, \ldots, n$, where $a(j)$ is the $j-$ th column of the matrix $A$. If we take $A$ equal to $\tilde{Q}$ we obtain the $H$ triangulation.

To compute the mesh of an AK triangulation, observe that diam $\sigma\left(\mathrm{y}^{1}, \pi\right)$ is independent of $\mathrm{y}^{1}$. So, by setting $\mathrm{y}^{1}=\underline{0}$ the next corollary follows immediately.

COROLLARY 3.2.3.

Clearly, mesh $A K_{\delta}=\delta$ mesh $A K$. So, the mesh can be made arbitrarily small by taking the grid size $\delta$ small enough.

Another triangulation well known from the literature but not of the form $\mathrm{AK}$ is the so-called "Union Jack" triangulation. It is a centrally symmetric triangulation due to Tucker (see Lefschetz [1949, page 140], cf. also Whitney [1957, page 358], Todd [1977a] and Kojima [1978b]).

\subsection{MEASURES FOR THE EFFICIENCY OF TRIANGULATIONS.}

A first measure used for the efficiency of a triangulation of the unit cube is the number of simplices in which the unit cube is triangulated. The $\mathrm{K}$ and $\mathrm{H}$ triangulations both subdivide the $\mathrm{n}$-dimensional unit cube in $n$ ! simplices. However, Saigal [1977a] experienced that in particular for large $\mathrm{n}$ the $\mathrm{K}$ triangulation performs much better than the $\mathrm{H}$ triangulation. This fact is not unexpected since the $\mathrm{H}$ triangulation yields many shapes of simplices, whereas the $\mathrm{K}$ triangulation results in one single shape, i.e. all simplices of this triangulation are congruent. So, this measure is too rough and must be rejected. We mention however, that Mara [1972] succeeded in finding a triangulation which divides the unit cube in 5, 16 and 68 simplices for respectively $n=3,4$ and 5 . A second measure was given by Saigal, Solow and Wolsey [1975]. They defined the measure of a triangulation. of the unit cube as its diameter 
which is the maximum of the minimal length nath between any pair of boundary facets of the triangulation. The length of a path between two facets is the number of simplices met by the path. Saigal [1977a] stated that the diameter of the $\mathrm{H}$ triangulation was of the order or at least $\mathrm{n}^{3}$, whereas the diameter of the $K$ triangulation is equal to $\frac{1}{2} n(n-1)+1$. A more sophisticated measure which can be calculated for triangulations of $\mathrm{R}^{\mathrm{n}}$ was introduced by Todd [1976b, 1978a]. This measure is called the average directional density and is roughly the average number of simplices met by straight lines per unit length. Formally, for some triangulation $G$ of $R^{n}$ denote, for $x, d \in R^{n}$ and $t>0$, the ratio between the number of simplices of $G$ intersecting the line segment $[x, x+t d]$ and $t$ by $N(G, x, d, t)$. Denoting by $N(G, d, t)$ the limit for $r \rightarrow \infty$ of the average of $N(G, x, d, t)$ for $x$ uniformly distributed in $\left\{y \in R^{n} \mid\|y\|<r\right\}$, the directional density of $G$ with direction $d$, to be denoted by $N(G, d)$, is the limit of $N(G, d, t)$ for $t \rightarrow \infty$ provided that both limits exist. Finally, the average directional density $N(G)$ of $G$ is the average of $N(G, d)$ for d uniformly distributed on the unit ball.

In the following, $A$ is a nonsingular $n \times n$ matrix and $b(j)$ is the $j$-th row of $A^{-1}$. Todd proved the following theorem.

THEOREM 3.3.1. The average directional density of the triangulation $\mathrm{AK}_{\delta}$ is

$$
N\left(A K_{\delta}\right)=\delta^{-1}\left\{\sum_{j=1}^{n}\|b(j)\|+\sum_{j=1 i=1}^{n} \sum_{i=1}^{j-1}\|b(i)-b(j)\|\right\} g_{n^{\prime}}
$$

where $g_{n}=2 \Gamma\left(\frac{1}{2} n\right) /\left\{(n-1) \sqrt{ } \pi \Gamma\left(\frac{1}{2} n-\frac{1}{2}\right)\right\}$.

From this theorem the next coroljary follows immediately.

COROLLARY 3.3 .2 .

$$
\begin{aligned}
& N\left(K_{\delta}\right)=\delta^{-1}\{n+n(n-1) / \sqrt{ } 2\} g_{n^{\prime}} \\
& N\left(H_{\delta}\right)=\delta^{-1}\left\{\sum_{j=1}^{n}(n-j+1) \sqrt{j}\right\} g_{n} .
\end{aligned}
$$

An optimal triangulation according to the average directional density is unknown. However, Todd [1978a] gave a lower bound for the average directional density of a triangulation of the form AK. Since the average directional density depends on the mesh, we will restrict ourselves 
to triangulations with mesh equal to or less than $\sqrt{ }$.

LEMMA 3.3.3. For any triangulation AK with mesh $A K \leq \sqrt{ }$ holds

$$
N(A K)>\left(4 n^{2} / 25 \sqrt{ } 5\right) g_{n}
$$

This result was strengthened by Van der Laan and Talman [1980a] for triangulations within a subclass $A$ of AK triangulations.

DEFINITION 3.3.4. An AK triangulation of $R^{n}$ belongs to the class $A$ if for some $\alpha>n-1, a_{i i}=\alpha, i=1, \ldots, n$ and $a_{i j}=-1, i \neq j$.

In chapter 6 it will be motivated that elements of this subclass are very suitable for use in fixed point algorithms, see also van der Laan and Talman [1980a] and Talman [1980]. An element of this class with grid size $\delta$ will be denoted by $\mathrm{A}(\alpha) \mathrm{K}_{\delta}$.

LEMMA 3.3.5. The mesh of an $A(\alpha) \mathrm{K}$ triangulation is equal to

$$
\operatorname{mesh} A(\alpha) K=\left[k\{\alpha-(k-1)\}^{2}+(n-k) k^{2}\right]^{\frac{1}{2}}
$$

where

$$
\begin{aligned}
k & =n & & \text { if } b \geq n \\
& =b & & \text { if } b \leq n-1 \text { and }\left\|y^{b+1}\right\| \geq\left\|y^{b+2}\right\| \\
& =b+1 & & \text { if } b \leq n-1 \text { and }\left\|y^{b+1}\right\|<\left\|y^{b+2}\right\|
\end{aligned}
$$

with $b$ the entier of $\left\{(\alpha+1)^{2} /(4+4 \alpha-2 n)\right\}$ and where $y^{1}, \ldots, y^{n+1}$ are the vertices of the simplex $\sigma(\underline{0}, \pi)$ with $\pi_{i}=i \quad i=1, \ldots, n$.

The proof can be found in Talman [1980]. Note that the $A(\alpha) K_{\hat{\delta}}$ triangulation with grid size $\delta=\alpha^{-1}$ converges to the $\mathrm{k}$ triangulation for $\alpha \rightarrow \infty$. The proof of the following lemma can be found in Van der Laan and Talman [1980 a] and Talman [1980].

LEMMA 3.3.6. For any $A(\alpha) K_{\delta}$ triangulation with mesh $A(\alpha) K_{\delta} \leq \sqrt{n}$ holds

$$
N\left(A(\alpha) K_{\delta}\right)>\left\{\frac{1}{2}(n-1)+(n-1)^{2} / \sqrt{ } 8\right\} g_{n}
$$


It is an open problem whether this lemma holds for all triangulations $\mathrm{AK}_{\delta}$ with mesh $A K_{\delta} \leq \checkmark n$.

Van der Laan and Talman [1980a] introduced a new measure for the efficiency of triangulations. This measure makes it possible to calculate the optimal triangulation within the class $A$. The measure called the SV-measure is based on both the total squared length of the one-faces of the simplices and the volume of these simplices.

DEFINITION 3.3.7. The SV-measure of a triangulation $G$, to be denoted by $\mathrm{SV}(G)$, is defined by

$$
\operatorname{SV}(G)=\sup _{\sigma \in \dot{G}}\left[\left\{\sum_{i=1}^{n} \sum_{j=i+1}^{n+1}\left\|y^{j}-y^{i}\right\|^{2}\right\}^{\frac{1}{2}} /\{\Delta \sigma\}^{1 / n}\right]
$$

where $\Delta \sigma$ denotes the volume of the simplex $\sigma=\sigma\left(y^{1}, \ldots, y^{n+1}\right)$.

The numerator reflects the total squared length of all one-faces of the simplex $\sigma$. By raising this term to the power a half and the term in the denominator to the power $1 / n$, we obtain a measure which is homogeneous of degree zero in the mesh. If the triangulation is of the form $A K$, each simplex $\sigma$ has the same volume $|\operatorname{det} A| / n !$. Roughly speaking the SV-measure of a triangulation is a measure for the number of grid points per unit cube with respect to the total squared length of all one-faces of the "worst" simplex $\sigma$. Clearly, for $n=2$, the SV-measure is minimized for a triangulation generating equilateral triangles. In Van der Laan and Talman [1980a] and Talman [1980] the following lemma's are proved.

LFMMA 3.3.8. The optimal triangulation within $A$ according to the SV-measure is the $A\left(\alpha^{*}\right) K$ triangulation with $\alpha^{*}=n+\sqrt{n+1}$.

LEMMA 3.3.9. The SV-measures of the $\mathrm{K}, \mathrm{H}$ and $\mathrm{A}\left(\alpha^{*}\right) \mathrm{K}$ triangulations are a) $\operatorname{sV}(\mathrm{K})=r_{\mathrm{n}} \sqrt{2}$

b) $\operatorname{SV}(\mathrm{H})=r_{n}\{3 n /(n+1)\}^{\frac{1}{2}}$ if $\mathrm{n}$ is even $=r_{n}\left\{3\left(n^{3}+2 n^{2}-n+2\right) / n(n+1)(n+2)\right\}^{\frac{1}{2}}$ if $n$ is odd c) $\operatorname{SV}\left(A\left(\alpha^{*}\right) K\right)=r_{n}(n+1)^{1 / 2 n}$, where $r_{n}=\{n(n+1)(n+2) / 12\}^{\frac{1}{2}}(n !)^{1 / n}$. 
LEMMA 3.3.10. The mesh of the $A\left(\alpha^{*}\right) \mathrm{K}_{\delta}$ triangulation is equal to

$$
\begin{aligned}
& \operatorname{mesh} A\left(\alpha^{*}\right) K_{\delta}=\frac{1}{2} \delta(1+\sqrt{n+1})(n+1) \quad \text { if } n \text { is odd } \\
& =\frac{1}{2} \delta(1+\sqrt{n+1}) \quad \text { if } n \text { is even. }
\end{aligned}
$$

LEMMA 3.3.11. The average directional density of the $A\left(\alpha^{*}\right) \mathrm{K}_{\delta}$ triangulation is equal to

$$
\begin{array}{rlrl}
N\left(A\left(\alpha^{*}\right) K_{\delta^{*}}\right) & =g_{n}\{n(n+1) / 8\}^{\frac{1}{2}}(n+1) & & \text { if } n \text { is odd } \\
& =g_{n}\{n(n+1) / 8\}^{\frac{1}{2}}\{n(n+2)\}^{\frac{1}{2}} & \text { if } n \text { is even. }
\end{array}
$$

where $\delta^{*}$ is taken such that mesh $A\left(\alpha^{*}\right) K_{\sigma}{ }^{*}=\sqrt{ }$.

Note that $\mathrm{N}\left(\mathrm{A}^{-}\left(\alpha^{*}\right) K_{\delta^{*}}\right)$ converges to the lower bound $g_{n} n^{2} / \sqrt{ } 8$ of lemma 3.3.6 as $\mathrm{n} \rightarrow \infty$. Furthermore, the $A\left(\alpha^{*}\right) \mathrm{K}$ triangulation has the nice properties that the barycenter of any simplex has the same distance $\{n(n+2)\}^{\frac{1}{2}}(1+\sqrt{n+1}) / 2 \sqrt{ } 3$ to each vertex of the simplex and that for any simplex $\sigma\left(y^{1}, \pi\right),\left\|y^{j}-y^{i}\right\|=\left\|y^{j-k}-y^{i-k}\right\|$ for all $1 \leq k<i<j \leq n+1$. These properties suggest to consider this triangulation of $R^{n}$ as the generalization of the equilateral triangulation of $\mathrm{R}^{2}$ in the sense that the triangulation yields simplices which are as "round" as possible.

\subsection{TRIANGULATIONS OF $\mathrm{S}^{\mathrm{n}}$ and $\mathrm{T}^{\mathrm{n}}$.}

A familiar triangulation of $\mathrm{s}^{\mathrm{n}}$ is given in the following definition.

DEFINITION 3.4.1. (Standard triangulation of $\mathrm{s}^{\mathrm{n}}$ ).

The standard triangulation of $\mathrm{S}^{\mathrm{n}}$ with grid size $\delta$ is the collection of all simplices $\sigma\left(y^{1}, \pi\right)$ with vertices $y^{1}, \ldots, y^{n+1}$ such that

a) $\delta^{-1}$ is a positive integer $m$, and each component of $y^{1}$ is a multiple of $\delta$

b) $\pi=\left(\pi_{1}, \ldots, \pi_{n}\right)$ is a permutation of the elements of $I_{n}$

c) $y^{i+1}=y^{i}+\delta q\left(\pi_{i}\right) \quad i=1, \ldots, n$,

where $q(j)$ is the $j-$ th column of the $(n+1) \times n$ matrix $Q$ 


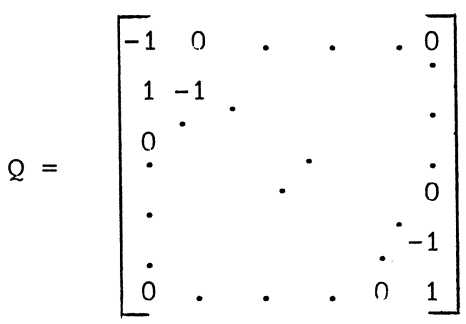

As proved by Todd [1976a] the mesh of the standard triangulation $Q$ with grid size $\delta$ is equal to mesh $\Omega_{\delta}=\delta \sqrt{\mathrm{n}+1}$ if $\mathrm{n}$ is odd and mesh $\Omega_{\delta}=\delta \sqrt{\mathrm{n}}$ if $\mathrm{n}$ is even. The following lemma has been proved in Van der Laan and Talman [1980a] and Talman [1980].

LEMMA 3.4.2. For the standard triangulation of $\mathrm{s}^{\mathrm{n}}$ holds

$$
\begin{array}{rlrl}
\operatorname{SV}(0) & =r_{n} \sqrt{ }\left\{\left(n^{3}+3 n^{2}-n+5\right) /\left(n^{3}+3 n^{2}+2 n\right)\right\}^{\frac{1}{2}}(n+1)^{-1 / 2 n} & \text { if } n \text { is odd } \\
& =r_{n} \sqrt{ } 3(n+1)^{-1 / 2 n} & & \text { if } n \text { is even }
\end{array}
$$

Other triangulations of $\mathrm{S}^{\mathrm{n}}$ include a triangulation related to the Union Jack triangulation of $\mathrm{R}^{\mathrm{n}}$ and the iterated barycentric subdivision (see Todd [1976a]).

We now discuss some triangulations of $T^{n}$, the affine hull of $s^{n}$.

DEFINITION 3.4.3. Let $z$ be an arbitrarily chosen point of $T^{n}$. Then the standard triangulation $Q$ of $\mathrm{T}^{\mathrm{n}}$ with respect to $\mathrm{z}$ with grid size $\delta$ is the collection of all simplices $\sigma\left(y^{1}, \pi\right)$ with vertices $y^{1}, \ldots, y^{n+1}$ such that
a) $y^{1}=z+\sum_{i=1}^{n} \alpha_{i} q(i)$ where $\alpha_{i}$ is a multiple of $\delta$ for all $i$
b) $\pi=\left(\pi_{1}, \ldots, \pi_{n}\right)$ is a permutation of the elements of $I_{n}$
c) $y^{i+1}=y^{i}+\delta q\left(\pi_{i}\right) \quad i=1, \ldots, n$.

of course the mesh of this triangulation is equal to the mesh of the standard triangulation of $\mathrm{s}^{\mathrm{n}}$. Also lemma 3.4 .2 holds for this triangulation of $T^{n}$. Observe that $S V(Q) / r_{n}$ converges to $\sqrt{ } 3$ for $n \rightarrow \infty$ as is also the case for the $H$ triangulation of $R^{n}$. Since $S V(H)$ is much higher than the SV-measure of the $A\left(\alpha^{*}\right) K$ triangulation of $R^{n}$, it seems to be worthwhile 
to look for a triangulation of $\mathrm{T}^{\mathrm{n}}$ whose $\mathrm{SV}$-measure is comparable with $\operatorname{SV}\left(A\left(\alpha^{*}\right) K\right)$.

A class of triangulations of $\mathrm{T}^{\mathrm{n}}$ can be defined in the following way. Let $A$ be an $(n+1) \times n$ matrix of rank $n$ such that $\Sigma_{i=1}^{n+1} a_{i j}=0$ for all $j=1, \ldots, n$. Again, let $\mathrm{z}$ be an element of $\mathrm{T}^{\mathrm{n}}$. The $\mathrm{A}$ triangulation of $\mathrm{T}^{\mathrm{n}}$ with grid size $\delta$ is defined as the set of all simplices $\sigma\left(y^{1}, \pi\right)$ such that $y^{1}=z+\sum_{i=1}^{n} \alpha_{i} a(i)$ where $\alpha_{i}$ is a multiple of $\delta$ for all $i$, $\pi=\left(\pi_{1}, \ldots, \pi_{n}\right)$ is a permutation of the elements of $I_{n}$ and $y^{i+1}=y^{i}+\delta a\left(\pi_{i}\right), i=1, \ldots, n$. Clearly mesh $A=\max _{S \subset I_{n}}\left\|\sum_{j \in S} a(j)\right\|$.

In Van der Laan and Talman [1980a] and Talman [1980] the following triangulation is proposed for use in fixed point algorithms. This triangulation is defined by the matrix

$$
T=\left[\begin{array}{cccccc}
-\mathrm{n} & 1 & \cdot & \cdot & \cdot & 1 \\
1 & -\mathrm{n} & & & & \cdot \\
\cdot & \cdot & & & \cdot \\
\cdot & & \cdot & \cdot & 1 \\
\cdot & & & & \cdot-\mathrm{n} \\
1 & \cdot & \cdot & \cdot & 1
\end{array}\right] .
$$

The simplices of this triangulation have the same nice properties as the simplices of the $A\left(\alpha^{*}\right) K$ triangulation of $R^{n}$. In particular these two triangulations have both $r_{n}(n+1)^{1 / 2 n}$ as SV-measure. We return to this triangulation of $\mathrm{T}^{\mathrm{n}}$ in chapter 5 .

To use a triangulation of $T^{n}$ in algorithms to compute a fixed point on $S^{n}$ it may be necessary to extend the function or mapning from $s^{n}$ into itself to a mapping from $T^{\mathrm{n}}$ to $\mathrm{s}^{\mathrm{n}}$.

\subsection{REPLACEMENT STEP.}

In this section we describe the replacement step between two fulldimensional adjacent simplices. Let $\sigma$ and $\bar{\sigma}$ be two of such simplices. Then $\bar{\sigma}$ is obtained from $\sigma$ by replacing a vertex of $\sigma$ by a new vertex. The rule to obtain this new vertex is quite simple when a regular triangulation is used. 
Let $P$ be a triangulation matrix e.g. $P$ is an $n \times n$ matrix to triangulate $R^{n}$ or an $(n+1) \times n$ matrix to triangulate $T^{n}$. Let $\sigma\left(y^{1}, \pi\right)$ be a simplex of the triangulation, so $\mathrm{y}^{1}$ is a grid point of the triangulation and $y^{i+1}=y^{i}+\delta p\left(\pi_{i}\right), \quad i=1, \ldots, n$, where $\delta$ is the grid size and $p(j)$ the $j$-th column of $P$. Then the rule to obtain the simplex $\bar{\sigma}\left(\bar{y}^{-1}, \bar{\pi}\right)$ by replacing the vertex $y^{i}$ of $\sigma\left(y^{1}, \pi\right)$ is given in table 3.5.1.

TABLE 3.5.1. $i$ is the index of the vertex to be replaced.

\begin{tabular}{l|l|l} 
& $\bar{y}^{-1}$ becomes & $\bar{\pi}_{\text {becomes }}$ \\
\hline$i=1$ & $y^{1}+\delta p\left(\pi_{1}\right)$ & $\left(\pi_{2}, \ldots, \pi_{n}, \pi_{1}\right)$ \\
$2 \leq i \leq n$ & $y^{1}$ & $\left(\pi_{1}, \ldots, \pi_{i-2}, \pi_{i}, \pi_{i-1}, \pi_{i+1}, \ldots, \pi_{n}\right)$ \\
$i=n+1$ & $y^{1}-\delta p\left(\pi_{n}\right)$ & $\left(\pi_{n}, \pi_{1}, \ldots, \pi_{n-1}\right)$
\end{tabular}

It is easy to see that the facet $\tau\left(y^{1}, \ldots, y^{i-1}, y^{i+1}, \ldots, y^{n+1}\right)$ is indeed a common facet of $\sigma\left(y^{1}, \pi\right)$ and $\bar{\sigma}\left(\bar{y}^{1}, \bar{\pi}\right)$. For instance if $i=1$ $\bar{y}^{i}=y^{i+1}, i=1, \ldots, n$, whereas $\bar{y}^{n+1}=y^{n+1}+\delta p\left(\pi_{1}\right)$.

Although some modifications can be necessary, table 3.5 .1 will be used in the algorithms to be treated in this monograph. Triangulations in which the replacement step can be described by table 3.5 .1 are called triangulations by reflecting since the new vertex is obtained by reflecting the old vertex with respect to the common facet. A full description of triangulations by reflecting can be found in Allgower and Georg [1980?. 
CHAP

ALGORITHMS FOR APPROXIMATING FIXED POINTS

\subsection{INTRODUCTION.}

In this chapter we consider some algorithms for computing a fixed point of a function or mapping from a set into itself where both integer and vector labelling will be treated. A first algorithm applied on the unit simplex was introduced in 1967 by H.E. Scarf. This algorithm is based on the concept of primitive sets rather than using a simplicial subdivision. In 1973 Scarf described a method for using the standard triangulation of the unit simplex. Earlier in 1968 and 1969 Kuhn developed already two methods to compute fixed points on the unit simnlex which both were based on the standard triangulation. The first method adds a layer of artificially labelled points to $s^{n}$ and starts with an $(n-1)-s i m p l e x$ on the boundary, whereas the other one uses no extra points, but starts with one of the vertices of the unit simplex. Analogous methods for computing fixed points of a continuous function from $\mathrm{R}_{+}^{\mathrm{n}}$ to itself can be easily derived. Whereas all these methods were introduced at first for integer labelling, an algorithm with vector labelling to compute a fixed point of an upper-semicontinuous mapping from a convex compact set into itself was developed by Hansen and Scarf in 1969. This method was based on primitive sets. However, Eaves [1971] developed an algorithm using a triangulation of a simplex containing the convex compact set.

The algorithms mentioned above all suffer from computational inefficjency because the start is made outside the region of interest while the grid size is kept fixed throughout the algorithm. This means that if for a given grid size an approximation is found and one wants to restart the method with a finer grid to obtain a better approximation, the method must be started again outside the region of interest. So all information about the fixed point is lost.

A number of algorithms to avoid this disadvantage were developed during the last decennium. One method is due to Merrill [1971,1972] and was 
independently found by Kuhn and Mackinnon [1975] (see also Lüthi [1975] and Fisher, Gould and Tolle [1977]). 'This algorithm can be applied both on $S^{n}$ and $R^{n}$ and is called the sandwich method. It can start anywhere. So, if this method is applied for a decreasing sequence of grid sizes one can choose in each stage the starting point in such a way that it is the most appropriate one by considering the information obtained from the approximation in the previous stage. An other method which takes advantage of the information already obtained in the course of the algorithm was introduced by Eaves [1972] for application on $S^{\mathrm{n}}$ and by Eaves and Saigal [1972] for $\mathrm{R}^{\mathrm{n}}$. In this method, called the homotopy algorithm, the grid size is automatically decreased with a factor of at most two. Both methods were introduced with vector labelling to compute a fixed point of an u.s.c. mapping.

This chapter is organized as follows. In section 2 we discuss the concept of integer labelling and we present some theorems about the accuracy of an approximation. In the sections 3-7 we treat the algorithms mentioned above for integer labelling. The algorithm of scarf is given in section 3 , the methods of Kuhn including a unified approach of his methods are given in section 4 , the analogous methods on $R_{+}^{n}$ in section 5 , the sandwich method in section 6 and the homotopy algorithm in section 7. In section 8 we present the concept of vector labelling. The basic algorithm of Eaves is presented in section 9 while the Sandwich method and the homotopy algorithm using vector labelling are described in section 10 for mappings on $R^{n}$. The computation of a fixed point of a mapping on $S^{n}$ is discussed briefly in section 11 . In section 12 we give some concluding remarks.

\subsection{INTEGER LABELLING AND APPROXIMATION.}

In chapter 2 it was proved that a continuous function from $s^{\mathrm{n}}$ into itself has at least one fixed point by taking a sequence of triangulations $\left\{G_{k}, k=1,2, \ldots\right\}$ with mesh $G_{k} \rightarrow 0$ if $k \rightarrow \infty$ and by labelling each grid point $x$ by an integer label $\ell(x)$. Now we give a labelling rule which is more appropriate to be used in algorithms for computing a fixed point.

DEFINITION 4.2.1. (Standard intecer labelling rule on $S^{\mathrm{n}}$ ) A point $x$ in $s^{n}$ receives the label $\ell(x)=i$, if

$$
i=\min \left\{j \in I_{n+1} \mid f_{j}(x)-x_{j}=\min _{m \in I_{n+1}} f_{m}(x)-x_{m} \text { and } x_{j}>0\right\} \text {. }
$$


Clearly there exists always such an index. Note that $x_{j}>0$ is not really a restriction since if $f_{j}(x)-x_{j}=\min _{m \in I_{n+1}} f_{m}(x)-x_{m}$ and $x_{j}=0$ for some $j$, then $x$ must be a fixed point. We call a labelling rule which satisfies the condition $\ell(x) \neq i$ if $x_{i}=0$ proper. By Sperner's lemma, for any triangulation $G_{k}, k=1,2, \ldots$ there exists a completely labelled simplex $\sigma_{k}$ of $G_{k}$ if the labelling rule is proper. Before introducing algorithms which generate a completely labelled simplex, we show how close an arbitrarily chosen point $x$ in a completely labelled simplex is to its image $f(x)$.

LEMMA 4.2.2. Let $G$ be a triangulation of $S^{\mathrm{n}}$ and let $\varepsilon, \delta>0$ be such that for every simplex $\sigma$ of $G$

$$
\max _{i \in I_{n+1}}\left|f_{i}(x)-f_{i}(y)\right|<\varepsilon \quad \text { for all } x, y \in \sigma
$$

and

$$
\max _{j \in I_{n+1}}\left|x_{i}-y_{i}\right|<\delta \quad \text { for all } x, \underline{y} \in \sigma .
$$

Then, for any completely labelled simplex $\sigma^{*}$ of $G$ and $x^{*} \epsilon \sigma^{*}$,

$$
\max _{i \in I_{n+1}}\left|f_{i}\left(x^{*}\right)-x_{i}^{*}\right|<n(\varepsilon+\delta) .
$$

PROOF. Let $w^{i}$ be the vertex of $\sigma^{*}$ with label $i, 1 \leq i \leq n+1$. Clearly $\overline{f_{i}\left(w^{i}\right)-w_{i}^{i}}$ is nonpositive for all $i$. Hence, for all $i$,

$$
f_{i}\left(x^{*}\right)-x_{i}^{*}=\left(f_{i}\left(x^{*}\right)-f_{i}\left(w^{i}\right)\right)+\left(f_{j}\left(w^{i}\right)-w_{i}^{i}\right)+\left(w_{i}^{i}-x_{i}^{*}\right)<\varepsilon+\delta .
$$

On the other hand, since $\sum_{j=1}^{n+1}\left(f_{j}\left(x^{*}\right)-x_{j}^{*}\right)=0$, we have for all $i$,

$$
f_{i}\left(x^{*}\right)-x_{i}^{*}=-\sum_{j \neq i}\left(f_{j}\left(x^{*}\right)-x_{j}^{*}\right)>-n(\varepsilon+\delta) \text {. }
$$

Combining these two inequalities we get the desired result. 
The lemma guarantees that a completely labelled simolex in $S^{\mathrm{n}}$ is indeed an approximation of a fixed point if the stanclard labelling rule is used. Consider now a continuous function $f$ from $R^{n}$ to $R^{n}$ and assume that this function has a fixed point (c.f. lemma 2.6.2). The standard integer labelling on $\mathrm{R}^{\mathrm{n}}$ is defined as follows.

DEFINITION 4.2.3. (Standard integer labelling on $\mathrm{R}^{\mathrm{n}}$ ). For some $x \in R^{n}$, let $i=\min \left\{j \in I_{n} \mid f_{j}(x)-x_{j}=\max _{m \in I_{n}} f_{m}(x)-x_{m}\right\}$. Then $x$ receives
the label $l(x)$ with

$$
\begin{aligned}
& \ell(x)=i \quad \text { if } f_{i}(x)-x_{i} \geq 0 \\
& n+1 \text { otherwise. }
\end{aligned}
$$

The next lemma gives the accuracy of the approximation of a fixed point when a completely labelled simplex $\sigma^{*}$ is found.

LEMMA 4.2.4. Let the triangulation $G$ of $R^{n}$ be such that $\max _{i \in I}\left|x_{i}-y_{i}\right|<\delta$ for all $x, y \in \sigma^{*}$ and let $\varepsilon>0$ be such that $i \in I_{n}$

$\max _{i \in I_{n}}\left|f_{i}(x)-f_{i}(y)\right|<\varepsilon$ for all $x, y \in \sigma^{*}$. Then for all $x^{*}$ in $\sigma^{*}$

$$
\max _{i \in I_{n}}\left|f_{i}\left(x^{*}\right)-x_{i}^{*}\right|<\varepsilon+\delta \text {. }
$$

PROOF. Again, let $w^{i}$ be the vertex of $\sigma^{*}$ with $\ell\left(w^{i}\right)=i, i=1, \ldots, n+1$. Clearly, $f_{i}\left(w^{i}\right)-w_{i}^{i} \geq 0$ for all $1 \leq i \leq n$ and $f_{j}\left(w^{n+1}\right)-w_{j}^{n+1}<0, j \in I_{n}$. Hence, for $i=1, \ldots, n$

$$
f_{i}\left(x^{*}\right)-x_{i}^{*}=\left(f_{i}\left(x^{*}\right)-f_{i}\left(w^{i}\right)\right)+\left(f_{i}\left(w^{i}\right)-w_{i}^{i}\right)+\left(w_{i}^{i}-x_{i}^{*}\right)>-(\varepsilon+\delta)
$$

and

$$
f_{i}\left(x^{*}\right)-x_{i}^{*}=\left(f_{i}\left(x^{*}\right)-f_{i}\left(w^{n+1}\right)+\left(f_{i}\left(w^{n+1}\right)-w_{i}^{n+1}\right)+\left(w_{i}^{n+1}-x_{i}^{*}\right)<\varepsilon+\delta .\right.
$$

So, if the standard labelling rule is used, a completely labelled simplex in $\mathrm{R}^{\mathrm{n}}$ yields an approximation of a fixed point. 


\subsection{SCARF'S ALGORITHM.}

The original algorithm of Scarf $[1967 \mathrm{~b}\rceil$ is based on the concept of primitive sets in stead of a triangulation of a simplex. Moreover, a labelling rule different from that of definition 4.2.1 was used. However, in this section we will follow Todd [1976a] using the standard labelling. To describe the algorithm we define the set $\tilde{s}^{n}$ by

$$
\tilde{S}^{n}=\left\{x \in R^{n+1} \mid \sum_{i=1}^{n+1} x_{i}=1 \text { and } x_{i} \leq 2, i \in I_{n+1}\right\} \text {. }
$$

$\tilde{S}^{n}$ is the $n$-simplex with vertices $y^{i}$ defined by $y^{i}=2 e-(2 n+1) e(i)$, $i=1, \ldots, n+1$, where $e$ is the vector with $e_{i}=1$ for all $1 \leq i \leq n+1$. Recall that $e(i)$ is the $i-t h$ unit vector of $R^{n+1}$.

In the next definition, let for some fixed $k$ with $k>n+1$ the points $y^{n+2}, \ldots, y^{k}$ be $k-n-1$ points in $s^{n}$ such that $y_{i} \neq y_{i}$ for all $m_{1}, m_{2} \geq n+1$, $\mathrm{m}_{1} \neq \mathrm{m}_{2}$ and for all $i \in \mathrm{I}_{\mathrm{n}+1}$.

DEFINITION 4.3.1. (Primitive sets).

For some $J \subset I_{k^{\prime}}|J|=n+1$, the set of $n+1$ points $\left\{y^{j} \mid j \in J\right\}$ forms $a$ primitive set if there is no $m$ with $n+2 \leq m \leq k$ such that $y_{i}^{m}>\min _{j \in J} y_{i}^{j}$ for all $i$.

observe that the set $\left\{y^{j} \mid j \in J=I_{n+1}\right\}$ of vertices of $\tilde{S}^{n}$ does not form a primitive set. Scarf's algorithms is based on the following lemma (see Scarf $[1967 \mathrm{~b}])$.

\section{LEMMA 4.3.2.}

a) For any $i \in I_{n+1}$, there exists a unique index $j_{i}>n+1$, such that the set of points $\left\{y^{j} \mid j \in\left(I_{n+1} \cup\left\{j_{i}\right\}\right) \backslash\{i\}\right\}$ forms a primitive set.

b) If $\left\{\mathrm{y}^{j} \mid j \in J\right\}$ is a primitive set and $\mathrm{m}_{1} \in J$ is such that $\left\{\mathrm{y}^{j} \mid j \in J \backslash\left\{m_{1}\right\}\right\}$ is not a subset of $\left\{\mathrm{y}^{j} \mid j \in I_{n+1}\right\}$, then there is a unique $m_{2} \notin J$ such that $\left\{y^{j} \mid j \in\left(J \cup\left\{m_{2}\right\}\right) \backslash\left\{m_{1}\right\}\right\}$ forms a primitive set.

Scarf's algorithm searches for a completely labelled primitive set, i.e. a primitive set $\left\{\mathrm{y}^{j} \mid j \in \mathrm{J}\right\}$ such that all elements are differently labelled. observe that a completely labelled primitive set indeed provides an approximation of a fixed point.

To start the algorithm the points outside $s^{n}$, viz. $y^{1}, \ldots, y^{n+1}$ are 
labelled by $\ell\left(y^{1}\right)=n+1$ and $\ell\left(y^{i}\right)=i-1, i=2, \ldots, n+1$. From lemma 4.3.2.a there is a unique primitive set $\left\{y^{2}, \ldots, y^{n+1}, y^{j}\right\}$. Observe that $I_{n}=\left\{l\left(y^{j}\right), j=2, \ldots, n+1\right\}$. Starting with this primitive set the algorithm makes a search for label $n+1$. Therefore $\ell\left(y^{j_{1}}\right)$ is calculated. If $\ell\left(\mathrm{y}^{j_{1}}\right)=\mathrm{n}+1$, the set is completely labelled and the algorithm terminates. Otherwise the point $y^{m_{1}}$ where $m_{1}$ is the index such that $\ell\left(y^{m_{1}}\right)=\ell\left(y^{j_{1}}\right)$ is replaced by $\mathrm{y}^{\mathrm{m}_{2}}$. such that $\left\{\mathrm{y}^{2}, \ldots, \mathrm{y}^{\mathrm{m}_{1}-1}, \mathrm{y}^{\mathrm{m}_{2}}, \mathrm{y}^{\mathrm{m}_{1}+1}, \ldots, \mathrm{y}^{\mathrm{n}+1}, \mathrm{y}^{\mathrm{j}_{1}}\right\}$ is again a a primitive set. Continuing by calculating the label of the new point and replacing the point having this label the algorithm generates a path of "adjacent" primitive sets until label $n+1$ is found. Scarf proved that the method will terminate within a finite number of replacement steps since all steps are unique and feasible whereas the number of primitive sets is finite.

For the replacement step a search through all points $\mathrm{y}^{1}, \ldots, \mathrm{y}^{\mathrm{k}}$ is required, unless these points are chosen in a systematic way. This is indeed possible but then there is a direct correspondence between primitive sets and simplices of the standard triangulation of $S^{n}$ (see scarf [1973, chapter 7]). The algorithm becomes then very close to the variable dimension algorithm of Kuhn to be discussed in the next section.

\subsection{KUHN'S ALGORITHMS.}

Besides the pioneering work of Scarf, two algorithms based on the standard triangulation of $\mathrm{s}^{\mathrm{n}}$ and using the standard labelling rule were developed by Kuhn [1968] and [1969], (see also Todd [1976a]), namely the artificial start algorithm and the variable dimension algorithm.

Before we present these algorithms we give some definitions. Recall that $S^{n}$ is the n-dimensional unit simplex, $T^{n}$ the affine hull of $S^{n}$ and $S_{i}^{n}$ the $i$-th boundary of $s^{n}, i=1, \ldots, n+1$. Let $\delta$ be the grid size of the standard triangulation of $\mathrm{T}^{\mathrm{n}}$ as defined in definition 3.4 .3 such that $\mathrm{e}(1)$ is a grid point and $\delta^{-1}=\mathrm{m}$ is a positive integer.

\section{DEF INITION 4.4.1.}

a) $\bar{s}^{n}=\left\{x \in T^{n} \mid x_{i} \geq 0, i \in I_{n}\right.$ and $\left.x_{n+1} \geq-m^{-1}\right\}$.

b) $\bar{s}_{i}^{n}$ is the i-th boundary of $\bar{s}^{n} i$.e.

$$
\bar{S}_{i}^{\mathrm{n}}=\left\{x \in \bar{S}^{\mathrm{n}} \mid \mathbf{x}_{i}=0\right\}, i \in I_{n}
$$


and

$$
\bar{S}_{n+1}^{n}=\left\{x \in \bar{S}^{n} \mid x_{n+1}=-m^{-1}\right\}
$$

c) $s^{n}(k)=\left\{x \in S^{n} \mid x_{i}=0, i=k+1, \ldots, n+1\right\}, k=1, \ldots, n+1$.

Obviously, since $e(1)$ is a grid point of the triangulation of $\mathrm{T}^{\mathrm{n}}$, the collection of simplices $\sigma$ such that $\sigma n \bar{s}^{-n}=\sigma$ triangulates $\bar{s}^{-n}$. Moreover, $\bar{s}_{n+1}^{n}$ is triangulated (see theorem 2.3.6) and all grid points of $\bar{s}^{n}$ outside $s^{n}$ are lying in $\bar{s}_{n+1}^{n}$.

In the following a permutation of the elements of the set $I_{k}$ is denoted by $\pi\left(I_{k}\right)=\left(\pi_{1}, \ldots, \pi_{k}\right)$. So, a simplex $\sigma$ of the triangulations given in 3 is denoted by $\sigma=\sigma\left(y^{1}, \pi\left(I_{n}\right)\right)$. A k-simplex $\sigma$ with vertices $y^{1}, \ldots, y^{k+1}$.is also denoted by $\sigma\left(y^{1}, \ldots \ldots, y^{k+1}\right)$.

The artificial start algorithm.

To apply this algorithm the points of $\bar{S}_{n+1}^{n}$ are artificially labelled by

$$
\ell(x)=\min \left\{j \mid x_{j}=\max _{k \in I_{n+1}} x_{k}\right\} \text { if } x \in \bar{s}_{n+1}^{n} .
$$

The points of $s^{\mathrm{n}}$ are labelled according to definition 4.2.1. Clearly, $\ell(x) \neq n+1$ if $x \in \bar{s}_{n+1}^{n}$ and $\ell(x) \neq i$ if $x \in \bar{s}_{i}^{n} i=1, \ldots, n$. Kuhn assumed that $m$ is a multiple of $n$, say $m=$ pn. Let $\tau^{\circ}\left(\underline{y}^{1}, \pi\left(I_{n-1}\right)\right)$ be the $(n-1)-$ simplex in $\bar{S}_{\mathrm{n}+1}^{\mathrm{n}}$ defined by (see figure 4.4 .1 )
a) $y_{1}^{1}=(p+1) / m, y_{i}^{1}=p / m, i=2, \ldots, n, y_{n+1}^{1}=-m^{-1}$
b) $\pi_{i}=i \quad i=1, \ldots, n-1$.

DEFINITION 4.4.2. For $k \in I_{n+1}$, a $(k-1)$-simplex $\sigma$ is $I_{k}$-complete if the $k$ vertices of $\sigma$ carry all the labels $1, \ldots, \mathrm{k}$.

Note that an $I_{n+1}$-compicu 1 -simplex is a completely labelled simplex. The next lemma follows from the labelling rule on $\bar{S}_{n+1}^{n}$, defined just above. It is a special case of lemma 4.4 .8 to be proved hereafter.

LEMMA 4.4.3. The $(n-1)$-simplex $\tau^{\circ}\left(\mathrm{y}^{1}, \pi\left(I_{n-1}\right)\right)$ is the only $I_{n}$-complete simplex in $\bar{s}_{n+1}^{n}$. 
By theorem 2.3.5 there exists a unique n-simplex $\sigma^{0}\left(\mathrm{y}^{1}, \ldots, \mathrm{y}^{\mathrm{n}+1}\right)$ of $\overline{\mathrm{s}}^{\mathrm{n}}$ having $\tau^{0}$ as a facet. Clearly $\sigma^{0}\left(y^{1}, \ldots, y^{n+1}\right)=\sigma^{0}\left(y^{1}, \pi\left(I_{n}\right)\right)$ with $y^{1}$ as defined just above and $\pi_{i}=i, i \in I_{n}$. Observe that $y^{n+1}$ is the only vertex of $\sigma^{0}$ in $s^{n}$. Moreover, $y^{n+1}$ is the barycenter of $s_{n+1}^{n}$. The simplex $\sigma^{0}$ will be the starting simplex of the algorithm. From this simplex on a path of adjacent n-simplices $\sigma^{0}, \sigma^{1}, \sigma^{2}, \ldots$ is generated such that the common ( $n-1)$-dimensional facets are $I_{n}$-complete, until a completely labelled simplex is found, i.e. the algorithm terminates if a grid point having label $\mathrm{n}+1$ is generated. Remember that the replacement step between two adjacent simplices is described in section 3.5. The algorithm can be described as follows.

Step 0. Set $\sigma$ equal to $\sigma^{0}\left(y^{1}, \pi\left(I_{n}\right)\right)$ and $\bar{y}$ equal to $y^{n+1}$.

Step 1. Calculate $\ell(\bar{y})$. If $\ell(\bar{y})=n+1 ; \sigma$ is completely labelled and the algorithm terminates. Otherwise, there is exactly one vertex $y^{\mathbf{S}} \neq \bar{y}$ for which $\ell\left(y^{S}\right)=\ell(\bar{y})$.

Step 2. The simplex $\sigma\left(y^{1}, \pi\left(I_{n}\right)\right)$ is adapted according to table 3.5 .1 by replacing $y^{\mathrm{s}}$. Return to step 1 with $\bar{y}$ equal to the new vertex of $\sigma$.

We now prove that the algorithm indeed terminates. The arguments used will also play a crucial role in the further simplicial algorithms. Firstly, from lemma 4.4.3 and the fact that $l(x) \neq i$ if $x \in \bar{S}_{i}^{n}$, it follows that $\tau^{0}$ is the only $I_{n}$-complete $(n-1)$-simplex on the boundary of $\bar{s}^{n}$. Therefore, all the replacement steps are feasible, i.e. the new simplex is always a simplex of $\bar{s}^{n}$, unless the algorithm returns in $\sigma^{0}$ while $y^{n+1}$ has to be replaced. However, the algorithm can not return in an earlier generated simplex. Suppose the contrary and let $j$ be the index such that for some $i<j$, $\sigma^{i}=\sigma^{j}$ and for all $k, h<j, \sigma^{k} \neq \sigma^{h}$, i.e. $\sigma^{j}$ is the first simplex which was generated earlier. Then $\sigma^{j-1}$ and $\sigma^{j}=\sigma^{i}$ have a common $I_{n}$-complete facet. Clearly, any generated simplex has only two $I_{n}$-complete facets. Hence $\sigma^{j-1}$ must be equal to either $\sigma^{i-1}$ or $\sigma^{i+1}$. If $i \geq 1$ then $\sigma^{j-1} \neq \sigma^{i-1}$ since $\sigma^{k} \neq \sigma^{h}$ for all $k, h<j$. If $i=0, \sigma^{0}$ has only $\sigma^{1}$ as adjacent simplex such that the common facet is $I_{n}$-complete. So, in both cases $\sigma^{j-1}=\sigma^{i+1}$. Moreover $j-1=i+1$, again by that fact that $\sigma^{k} \neq \sigma^{h}$ for all $\mathrm{k}, \mathrm{h}<j$. Hence $j=i+2$ and $\sigma^{i}=\sigma^{i+2}$. Because of step 2 this is impossible. 
Consequently, a path of differently adjacent simplices in $\overline{\mathrm{S}}^{\mathrm{n}}$ is generated. Since the number of simplices is finite, the algorithm must terminate within a finite number of replacement steps with a completely labelled simplex in $\bar{S}^{n}$. Since $\ell(x) \neq n+1$ if $x \in \bar{S}_{n+1}^{n} \cup s_{n+1}^{n}$, this simplex must be a simplex in $s^{n}$. The algorithm is illustrated for the example of figure 2.4.2 in figure 4.4.1.

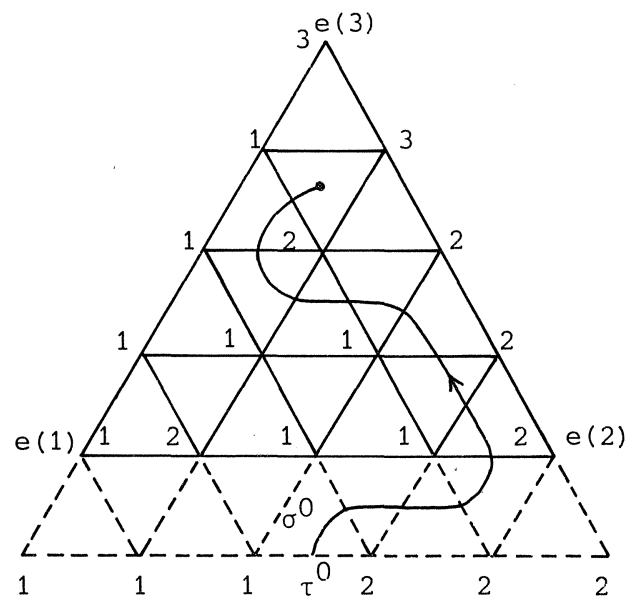

Figure 4.4.1. Kuhn's artificial start algorithm.

In other algorithms to be discussed in this section the arguments to prove non-cycling are very similar to the arguments above. Therefore, they are not repeated each time.

Kuhn's variable dimension algorithm.

This algorithm does not start with a full-dimensional simplex but with the single point $e(1)$. Recall the set $s^{n}(k)$ of definition 4.4.1.c and note that $s^{n}(1)=e(1)$ and that $s^{n}(n+1)=s^{n}$. Furthermore $s^{n}(n)=s_{n+1}^{n}$. Clearly points $x$ in $s^{n}(k)$ carry only labels of the set $I_{k}$ since $x_{i}=0$ for $i \geq k+1$. By theorem 2.3.6, $\mathrm{s}^{\mathrm{n}}(\mathrm{k})$ is triangulated in $(\mathrm{k}-1)$-simplices. It is obvious that $\mathrm{s}^{\mathrm{n}}(\mathrm{k})$ corresponds with $s^{k-1}$, for any $1 \leq k \leq n+1$, whereas a proper labelling on $s^{n}$ induces a proper labelling on $S^{n}(k)$. So, by sperner's lemma $S^{n}(k)$ has at least one $I_{k}$-complete simplex, $k=1, \ldots, n+1$. The algorithm starts with the $\{1\}$-complete zero-dimensional simplex $\{e(1)\}$ of $s^{n}(1)$. As soon as for some $k, k=1, \ldots, n$, an $I_{k}$-complete simplex in $S^{n}(k)$ is found, the algorithm continues with a path of $k$-simplices in $s^{n}(k+1)$ having $I_{k}$-complete common facets, until either an $I_{k+1}$-complete simplex in $S^{n}(k+1)$ is found or a 
simplex having an $I_{k}$-complete facet in $S^{n}(k)$ is generated. In the first case the dimension is increased, whereas in the latter case the dimension is decreased. The algorithm terminates if a completely labelled simplex is found. Formally, the steps are as follows.

Step 0. Set $k=1, y^{1}=e(1), \sigma=\sigma^{0}\left(y^{1}, \pi(\emptyset)\right)$ and $\bar{y}=y^{1}$.

step 1. Calculate $\ell(\bar{y})$. If $\ell(\bar{y})=k$, an $I_{k}$-complete simplex in $S^{n}(k)$ is found and go to step 3. Otherwise, there is exactly one vertex $\mathrm{y}^{\mathrm{S}} \neq \overline{\mathrm{y}}$ such that $\ell\left(\mathrm{y}^{\mathrm{S}}\right)=\ell(\overline{\mathrm{y}})$.

Step 2. If $s=k$ and $y_{k}^{k-1}=0$, the facet $\tau\left(y^{1}, \ldots, y^{k-1}\right)$ of $\sigma$ is an $I_{k-1}$ complete simplex in $S^{\mathrm{n}}(\mathrm{k}-1)$ and go to step 4 . Otherwise $\sigma\left(y^{1}, \pi\left(I_{k-1}\right)\right)$ is adapted according to table 4.4.2 by replacing $y^{S}$. Return to step 1 with $\bar{y}$ equal to the new vertex of $\sigma$.

Step 3. If $k=n+1$, a completely labelled simplex is found and the algorithm terminates. If $k<n+1, \pi\left(I_{k-1}\right)$ becomes $\pi\left(I_{k}\right)=$ $\left(\pi\left(I_{k-1}\right), k\right), \sigma$ becomes $\sigma\left(y^{1}, \pi\left(I_{k}\right)\right)$. Then $k$ becomes $k+1$ and return to step 1 with $\bar{y}=y^{k}=y^{k-1}+q(k-1) / m$.

Step 4. Set $\sigma$ equal to $\tau$ and set $\pi\left(I_{k-1}\right)$ equal to $\pi\left(I_{k-2}\right)=\left(\pi_{1}, \ldots, \pi_{k-2}\right)$. Then $\mathrm{k}$ becomes $\mathrm{k}-1$ and return to step 2 with $\mathrm{y}^{\mathrm{S}}$ equal to the vertex of $\sigma$ having label $\mathrm{k}$.

Table 4.4.2. $\mathrm{s}$ is the index of the vertex to be replaced.

\begin{tabular}{l|l|l} 
& $y^{1}$ becomes & $\pi_{k-1}\left(I_{k}\right)$ becomes \\
\hline$s=1$ & $y^{1}+q\left(\pi_{1}\right) / m$ & $\left(\pi_{2}, \ldots, \pi_{k-1}, \pi_{1}\right)$ \\
$2 \leq s \leq k-1$ & $y^{1}$ & $\left(\pi_{1}, \ldots, \pi_{s-2}, \pi_{s}, \pi_{s-1}, \pi_{s+1}, \ldots, \pi_{k-1}\right)$ \\
$s=k$ & $y^{1}-q\left(\pi_{k-1}\right) / m$ & $\left(\pi_{k-1}, \pi_{1}, \ldots, \pi_{k-2}\right)$
\end{tabular}

Note that table 4.4.2 is the analogon of table 3.5.1 adapted for the fact that the variable dimension $k-1$ is used in stead of fixed dimension $\mathrm{n}$. By the proper labelling all replacement steps are feasible, whereas also the increasing of the dimension in step 3 is feasible. Using analogous arguments as above the algorithm can never return in an earlier generated simplex. Therefore, within a finite number of iterations a completely labelled simplex is found. A more detailed proof will be given in chapter 5. In figure 4.4.2 the algorithm is illustrated for the example above. 


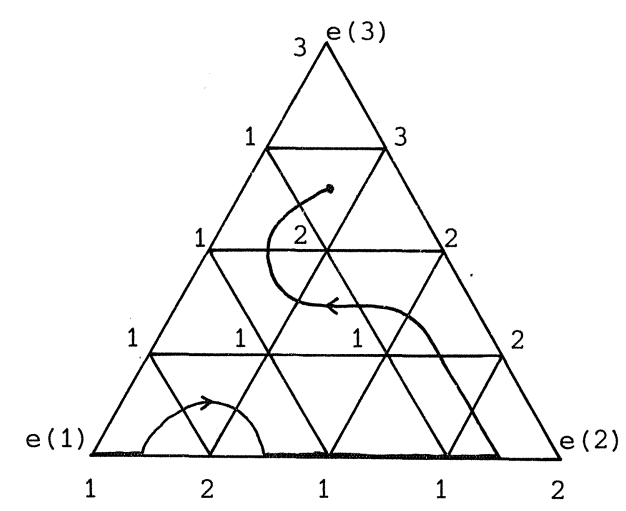

Figure 4.4.2. Kuhn's variable dimension algorithm.

It is clear that from a theoretical point of view the variable dimension algorithm can be implemented for any (not necessarily regular) triangulation of $\mathrm{s}^{\mathrm{n}}$. Since always a completely labelled $\mathrm{n}$-simplex will be generated we have the following corollary, known as Sperner's lemma.

COROLLARY 4.4.4. If the vertices of a triangulation of $s^{n}$ are properly labelled, then there exists at least one completely labelled n-simplex.

We will now show that the number of completely labelled simplices is odd. Suppose $\sigma_{1}$ is the completely labelled simplex generated by the variable dimension algorithm and let $\sigma_{2}\left(\bar{y}^{1}, \bar{\pi}\left(\tau_{n}\right)\right)$ be another completely labelled simplex (if any). Then we can apply the algorithm by a start in step 2 with $\mathrm{k}=\mathrm{n}+1, \sigma=\sigma_{2}, \mathrm{y}^{1}=\bar{y}^{-1}$ and $\pi\left(I_{n}\right)=\bar{\pi}\left(I_{n}\right)$ and $s$ equal to $i$ if $i$ is the index such that $\ell\left(y^{i}\right)=n+1$. Since the path between $\{e(1)\}$ and $\sigma_{1}$ is unique, by performing the algorithm the path of generated simplices does not cross the path between $\{e(1)\}$ and $\sigma_{1}$ and hence a new completely labelled simplex is found, say $\sigma_{3}$. The same holds if we pick up a completely labelled simplex $\sigma_{4}$ etc. So we have the following corollary, which is known as a strongex form of Sperner's lemma.

COROLLARY 4.4.5. If the vertices of a triangulation of $\mathrm{S}^{\mathrm{n}}$ are properly labelled, the number of completely labelled simplices is odd. 
We will return to this matter in chapter 7 .

The algorithms of Kuhn have the disadvantage that they must start on the boundary respectively in one of the corners of the unit simplex. So, information obtained by applying the algorithm with a coarse grid cannot be used for an application with a finer grid. The Sandwich method to be discussed in section 6 is related to the artificially start algorithm but can start anywhere. In chapter 5 we will introduce an algorithm related to the variable dimension algorithm and which can also start anywhere. It will appear that compared with the Sandwich method the latter algorithrn has the advantage that there are no artifjcially labelled points and that it generates a path of simplices of variable dimension, starting with only one point.

We conclude this section with a class of algorithms of which the two algorithms of Kuhn are the extreme cases.

A class of algorithms

Before presenting the algorithms we give some definitions. Recall that $T^{\mathrm{n}}$ is triangulated with grid size $\mathrm{m}^{-1}$ such that $\mathrm{e}(1)$ is a grid point and with $\mathrm{m}$ some positive integer.

\section{DEFINITION 4.4.6.}

a) $\bar{S}^{n}(k)=\left\{x \in T^{n} \mid x_{i} \geq 0, i \in I_{k^{\prime}} x_{k+1} \geq-m^{-1}, x_{i}=0, i=k+2, \ldots, n+1\right\} \quad k=1, \ldots, n$.

b) For $k=1, \ldots, n, \bar{s}_{i}^{n}(k)$ is the $i$-th boundary of $\bar{s}^{n}(k)$, i.e.

$$
\begin{aligned}
& \bar{S}_{i}^{n}(k)=\left\{x \in \bar{S}^{n}(k) \mid x_{i}=0\right\} \quad i=1, \ldots, k \\
& \bar{s}_{k+1}^{n}(k)=\left\{x \in \bar{S}^{n}(k) \mid x_{k+1}=-m^{-1}\right\} .
\end{aligned}
$$

Note that $\bar{S}^{n}(n)=\bar{S}^{n}$. It is obvious that $\bar{S}^{n}(k)$ is triangulated in $k$-simplices and that grid points of $\bar{s}^{n}(k)$ outside $s^{n}$ are points of $\overline{\mathrm{S}}_{\mathrm{k}+1}^{\mathrm{n}}(\mathrm{k})$. For given $\mathrm{k}$, the algorithm starts with a k-simplex of $\bar{s}^{-\mathrm{n}}(\mathrm{k})$ having an $I_{k}$-complete $(k-1)$-facet in $\bar{S}_{k+1}^{n}(k)$. Therefore, let $y$ be some grid point in $S^{n}(k)$ (see definition 4.4.1.c). Clearly $y \in \bar{S}^{n}(k)$, since $\mathrm{s}^{\mathrm{n}}(\mathrm{k}) \subset \overline{\mathrm{s}}^{\mathrm{n}}(\mathrm{k})$. Now the points of $\overline{\mathrm{s}}_{\mathrm{k}+1}^{\mathrm{n}}(\mathrm{k})$ are artificially labelled such that $\ell(z)=i$ if 


$$
i=\min \left\{j \mid y_{j}-z_{j}=\min _{h \in I_{n+1}} y_{h}-z_{h}\right\} .
$$

Of course, the points of $s^{\mathrm{n}}$ are labelled according to the standard rule. Let $\tau\left(w^{1}, \ldots, w^{k}\right)$ be the $(k-1)$-simplex of $\bar{s}_{k+1}^{r_{1}}(k)$ defined by

$$
w^{k}=y-q(k) / m \text { and } w^{i}=w^{i+1}-q(i) / m \quad i=k-1, \ldots, 1 \text {. }
$$

Then we have the following lemma.

LEMMA 4.4.7. The $(k-1)$-simplex $\tau$ is the only $I_{k}$-complete simplex in $\bar{s}_{\mathrm{k}+1}^{\mathrm{n}}(\mathrm{k})$.

PROOF. We first prove that $\tau$ is $I_{k}$-complete. Clearly, for $i=1, \ldots, k$,

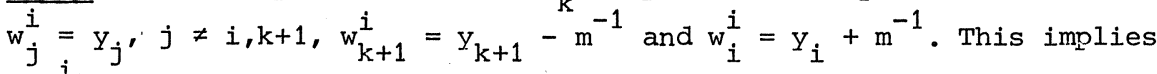
$\ell\left(w^{i}\right)=i$, so $\tau$ is $I_{k}$-complete. It remains to prove the second part of the lemma. Suppose that the simplex $\rho\left(u^{1}, \ldots, u^{k}\right)$ is an $I_{k}$-complete simplex in $\bar{s}_{k+1}^{n}(k)$. If for some $i, j, 1 \leq i, j \leq k, u_{j}^{i}=y_{j}-m^{-1}$, then $u_{j}^{h} \leq y_{j}$ for $h=1, \ldots, k$, contradicting that $\ell\left(u^{h}\right)=j$ for some $h$. Hence, $u_{j}^{i} \geq y_{j}$ for all $i, j, 1 \leq i, j \leq k$. Moreover, by the definition of $s^{n}(k)$ and $\bar{s}_{k+1}^{n}(k)$ we have that $y_{j}=u_{j}^{i}=0(j=k+2, \ldots, n+1 ; i=1, \ldots, k), y_{k+1}=0$ and $u_{k+1}^{i}=-m^{-1},(i=1, \ldots, k)$. Hence $\sum_{j=1}^{k} u_{j}^{i}=\Sigma_{j=1}^{k} y_{j}+m^{-1}$ for $i=1, \ldots, k$. It follows that for $i=1, \ldots, k$

$$
u_{\phi(i)}^{i}=y_{\phi(i)}+m^{-1} \text { for some index } \phi(i) \text { with } 1 \leq \phi(i) \leq k
$$

and

$$
u_{j}^{i}=y_{j^{\prime}} \quad \text { for } j \neq \phi(i), k+1
$$

Recall that the triangulation of $\overline{\mathrm{s}}_{\mathrm{k}+1}^{\mathrm{n}}(\mathrm{k})$ is induced by the standard triangulation of $T^{n}$. Therefore, since $\rho$ is a simplex of $\bar{S}_{k+1}^{n}(k)$, we must have that $u^{i+1}=u^{i}+q\left(\pi_{i}\right) / m, i=1, \ldots, k-1$, for some permutation $\pi\left(I_{k-1}\right)$. Let $h$ be the index such that $\pi_{h}=1$. Now assume $u_{1}^{1}=y_{1}$. Then $u_{1}^{h+1}=$ $y_{1}-m^{-1}$ which contradicts $u_{1}^{h+1} \geq y_{1}$. Consequently, $u_{1}^{1}=y^{1}+m^{-1}$ and hence $\phi(1)=1$ and $u_{i}^{1}=y_{i}, i=2, \ldots, k$. So $u^{1}=w^{1}$. Moreover $\pi_{1}$ must be equal to one, implying that $u_{2}^{2}=y_{2}+m^{-1}$, i.e. $\phi(2)=2$. Consequently $\phi(i)=\pi_{i}=i, i=1, \ldots, k-1$ which proves that $\rho=\tau$. 
It is obvious that lemma 4.4.7 implies lemma 4.4 .3 by taking $k=n$ and $y_{j}=p / m j=1, \ldots, n, y_{n+1}=0$.

We are now prepared to sketch the algorithm associated with a given $k^{*}$ and a given point $y$ being a grid point in $s^{n}\left(k^{*}\right)$. The method starts with the $\mathrm{k}$-simplex $\sigma^{0}\left(\mathrm{y}^{1}, \ldots, \mathrm{y}^{\mathrm{k}^{*}+1}\right)$, where $\mathrm{y}^{\mathrm{k}^{*}+1}=\mathrm{y}$ and where $\mathrm{y}^{1}, \ldots, \mathrm{y}^{\mathrm{k}^{*}}$ are the vertices of the simplex $\tau$ of lemma 4.4.7, i.e. $\sigma^{0}=\sigma^{0}\left(y^{1}, \pi\left(I_{k^{*}}\right)\right)$ with $\pi_{i}=i, i=1, \ldots, k^{\star}$. By applying Kuhn's artificial start algorithm, in which we take $k^{*}$ in stead of $n$, an $I_{k^{*}+1}$-complete simplex $\rho=\rho\left(v^{1}, \bar{\pi}\left(I_{k^{*}}\right)\right)$ of $s^{n}\left(k^{*}+1\right)$ mus.t be found within a finite number of steps. Then, we continue with the variable dimension algorithm by setting in step $0, k=k^{*}+2$, $\pi\left(I_{k^{*}+1}\right)=\left(\bar{\pi}_{1}, \ldots, \bar{\pi}_{k^{*}}, k^{*}+1\right), \sigma\left(y^{1}, \pi\left(I_{k^{*}+1}\right)\right)=\sigma\left(v^{1}, \pi\left(I_{k^{*}+1}\right)\right)$ and $\bar{y}=v^{k^{*}+2}$. Within a finite number of iterations, the algorithm either terminates with a completely labelled simplex or generates a simplex of $S^{n}\left(k^{*}+2\right)$ having an $\left(I_{k^{*}+1}\right)$-complete facet in $s^{n}\left(k^{*}+1\right)$. In the latter case the method proceeds again according to the artificial start method and generates simplices in $\bar{S}^{-n}\left(k^{*}\right)$ having $I_{k^{*}}$-complete common facets, until again an $\mathrm{I}_{\mathrm{k}^{*}+1}$-complete simplex of $\mathrm{S}^{\mathrm{n}}\left(\mathrm{k}^{*}+1\right)$ is found etc. Again using the fact that all steps are feasible and that cycling can not occur, a completely labelled simplex must be found within a finite number of steps. Observe that for $\mathrm{k}^{*}=\mathrm{n}$ we have Kuhn's artificial start algorithm whereas for $\mathrm{k}^{*}=1$ the method is just like the variable dimension algorithm.

4.5. ALGORITHMS TO COMPUTE FIXED POINTS ON $\mathrm{R}_{+}^{\mathrm{n}}$.

In this section a class of algorithms for computing a fixed point (if any) of a continuous function from $R_{+}^{n}$ to $R_{+}^{n}$ is presented. This class is analogous to the class of algorithms described in the previous section. An application of these algorithms is the nonlinear complementarity problem.

For all members of the class of algorithms, $R_{+}^{n}$ is triangulated by the $\mathrm{K}_{\delta}$-triangulation. For ease of notation we take $\delta=1$ in the discussion below. Analogous to the class of algorithms on $s^{n}$ we want to have a proper labelling, i.e. a labelling such that $\ell(x) \neq i$ if $x_{i}=0$. Therefore the standard labelling rule on $R^{n}$ is not appropriate and we define the following labelling rule. 
DEFINITION 4.5.1. (Labelling rule on $R_{+}^{n}$ ). For $x \in R_{+}^{n}$, let $i$ be the index such that $i=\min \left\{j \mid f_{j}(x)-x_{j}=\min _{h \in I_{n}} f_{h}(x)-x_{h}\right\}$.
Then

$$
\begin{aligned}
\ell(x) & =i & & \text { if. } f_{i}(x)-x_{i}<0 \\
& =0 & & \text { otherwise. }
\end{aligned}
$$

Note that the labelling rule is proper. In particular we have that $l(\underline{0})=0$. Furthermore it is easy to see that a completely labelled simplex yields an approximation of a fixed point. We call a k-simplex $\left(I_{k} \cup\{0\}\right)$-complete if the $k+1$ vertices carry all the labels $0,1, \ldots, k$.

We give now some definitions which are analogous to definition 4.4.6 and definition 4.4.1.cand which are helnful to describe the starting procedure.

\section{DEFINITION 4.5 .2 .}

a) $\bar{R}^{n}(k)=\left\{x \in R^{n} \mid x_{i} \geq 0, i \in I_{k}, x_{k+1} \geq-1\right.$ and $\left.x_{i}=0, i=k+2, \ldots, n\right\} \quad k=0, \ldots, n-1$.

b) For $k=0, \ldots, n-1, \bar{R}_{i}^{n}(k)$ is the $i-t h$ boundary of $\bar{R}^{n}(k)$, i.e.

$$
\begin{aligned}
& \bar{R}_{i}^{n}(k)=\left\{x \in \bar{R}^{n}(k) \mid x_{i}=0\right\} \quad i=1, \ldots, k ; \\
& \bar{R}_{k+1}^{n}(k)=\left\{x \in \bar{R}^{-n}(k) \mid x_{k+1}=-1\right\}
\end{aligned}
$$

c) $R^{n}(k)=\left\{x \in R^{n} \mid x_{i} \geq 0, i \in I_{k}\right.$ and $\left.x_{i}=0, i=k+1, \ldots, n\right\} \quad k=0, \ldots, n$.

The K-triangulation of $\mathrm{R}^{\mathrm{n}}$ implies that for given $\mathrm{k}=\mathrm{k}^{*}$ with $0 \leq \mathrm{k}^{*} \leq \mathrm{n}-1$, $\bar{R}^{n}\left(k^{*}\right)$ is triangulated in such a way that the grid points of $\bar{R}^{-n}\left(k^{*}\right)$ not in $\mathrm{R}_{+}^{\mathrm{n}}$ are points of $\mathrm{R}_{\mathrm{k}^{*}+1}^{\mathrm{n}}\left(\mathrm{k}^{*}\right)$.

Now, let $y$ be an arbitrarily chosen grid point of $R^{n}\left(k^{*}\right)$, i.e. $y_{i} \geq 0$ for $i \in I_{k^{*}}$. The algorithm associated with a given $k^{*}$ starts with the simplex $\sigma\left(y^{1}, \pi\left(I_{k^{*}+1}\right)\right)$ where $y^{k^{*}+2}=y$ and $y^{i}=y^{i+1}-e(i), i=1, \ldots, k^{*}+1$, i.e. $y^{1}=y-\Sigma_{i=1}^{k^{*}+1} e(i)$ and $\pi_{i}=i, i=1, \ldots, k^{*}+1$. To guarantee that the facet $\tau\left(y^{1}, \ldots \ldots, y^{*}+1\right)$ is the only $\left(I_{k^{*}} \cup\{0\}\right)$-complete simplex of $\overline{\mathrm{R}}_{\mathrm{k}^{*}+1}^{\mathrm{n}}\left(\mathrm{k}^{*}\right)$ the points of $\overline{\mathrm{R}}_{\mathrm{k}^{*}+1}^{\mathrm{n}}\left(\mathrm{k}^{*}\right)$ are artificially labelled by the following rule. Let $i$ be the index such that

$$
i=\min \left\{j \mid y_{j}-x_{j}-j /\left(k^{*}+1\right)=\min _{h \in I_{k}{ }^{*}} y_{h}-x_{h}-h /\left(k^{*}+1\right)\right\} .
$$


Then $\ell(x)=i$ if $y_{i}-x_{i}-i / k^{*}+1<0$ and $\ell(x)=0$ otherwise.

Following the proof of lemma 4.4.7 it follows immediately that $\tau$ is indeed the only $\left(I_{k^{*}} \cup\{0\}\right)$-complete simplex of $\bar{R}_{k^{*}+1}^{n}\left(k^{*}\right)$. In particular holds: $\ell\left(y^{i}\right)=i-1, i=1, \ldots, k^{*}+1$.

The algorithm proceeds as follows.

Step 0. Set $\mathrm{k}=\mathrm{k}^{*}+1, \sigma=\sigma\left(\mathrm{y}^{1}, \pi\left(\mathrm{I}_{\mathrm{k}^{*}+1}\right)\right)$ and $\overline{\mathrm{y}}=\mathrm{y}^{\mathrm{k}+1}\left(=\mathrm{y}^{\mathrm{k}^{*}+2}=\mathrm{y}\right)$.

Step 1. Calculate $l(\bar{y})$. If $\ell(\bar{y})=k$, an $\left(I_{k} \cup\{0\}\right)$-complete simplex of $R^{n}(k)$ is found and go to step 3. Otherwise there is exactly one vertex $\mathrm{y}^{\mathbf{S}} \neq \overline{\mathrm{y}}$ suoh that $\ell\left(\mathrm{y}^{\mathbf{S}}\right)=\ell(\overline{\mathrm{y}})$.

Step 2. If $\mathrm{s}=\mathrm{k}+1, \mathrm{y}_{\mathrm{k}}^{\mathrm{k}}=0$ and $\mathrm{k}>\mathrm{k}^{*}+1$, the facet $\tau\left(\mathrm{y}^{1}, \ldots, \mathrm{y}^{\mathrm{k}}\right)$ of $\sigma$ is an $\left(I_{k-1} \cup\{0\}\right)$-complete simplex of $R^{n}(k-1)$ and go to step 4 . Otherwise $\sigma$ is adapted according to table 4.5 .1 by replacing $\mathrm{y}^{\mathrm{S}}$. Return to step 1 with $\bar{y}$ equal to the new vertex of $\sigma$.

Step 3. If $k=n, a$ completely labelled simplex is found and the algorithm terminates. If $k<n, \pi\left(I_{k}\right)$ becomes $\pi\left(I_{k+1}\right)=\left(\pi\left(I_{k}\right), k+1\right), \sigma$ becomes $\sigma\left(y^{1}, \pi\left(I_{k+1}\right)\right)$. Then $k$ becomes $k+1$ and return to step 1 with $\bar{y}=y^{k+1}=y^{k}+e(k)$.

Step 4. Set $\sigma$ equal to $\tau$ and set $\pi\left(I_{k}\right)$ equal to $\pi\left(I_{k-1}\right)=\left(\pi_{1}, \ldots, \pi_{k-1}\right)$. Then $\mathrm{k}$ becomes $\mathrm{k}-1$ and return to step 2 with $\mathrm{y}^{\mathrm{S}}$ equal to the vertex having label $\mathrm{k}$.

Observe that table 4.5.1 is analogous to table 4.4 .2 and that it represents the replacement step between two adjacent $(k+1)$-simplices. Recall that $R^{n}(k)$ and $\bar{R}^{n}(k-1)$ are $k$ dimensional subspaces of $R^{n}$.

Table 4.5.1. $\mathrm{s}$ is the index of the vertex to be replaced.

\begin{tabular}{l|l|l} 
& $y^{1}$ becomes & $\pi\left(I_{k}\right)$ becomes \\
\hline$s=1$ & $y^{1}+e\left(\pi_{1}\right)$ & $\left(\pi_{2}, \ldots, \pi_{k}, \pi_{1}\right)$ \\
$2 \leq s \leq k$ & $y^{1}$ & $\left(\pi_{1}, \ldots, \pi_{s-2}, \pi_{s}, \pi_{s-1}, \pi_{s+1}, \ldots, \pi_{k}\right)$ \\
$s=k+1$ & $y^{1}-e\left(\pi_{k}\right)$ & $\left(\pi_{k}, \pi_{1}, \ldots, \pi_{k-1}\right)$.
\end{tabular}

The class of algorithms corresponds to the class of algorithms on $s^{n}$, described in the previous section. In particular, the case $k^{*}=n-1$ corresponds to Kuhn's artificial start algorithm on $\mathrm{s}^{\mathrm{n}}$, whereas the case $\mathrm{k}^{*}=0$ is the analogon of the variable dimension method on $\mathrm{s}^{\mathrm{n}}$. In the latter case the algorithm starts with the point $y=0$ of $R^{n}(0)$. 
By the proper labelling, it is easy to see that all replacement steps are feasible. Moreover, using the same arguments as for the artificial start algorithm of section 4, cycling cannot occur. Therefore the algorithm either terminates with a completely labelled simplex in $R_{+}^{n}$ or an infinite path of simplices going to infinity is generated. The algorithm is illustrated in figure 4.5 .1 for $n=2$ and $k^{*}=0$ respectively $k^{*}=1$.
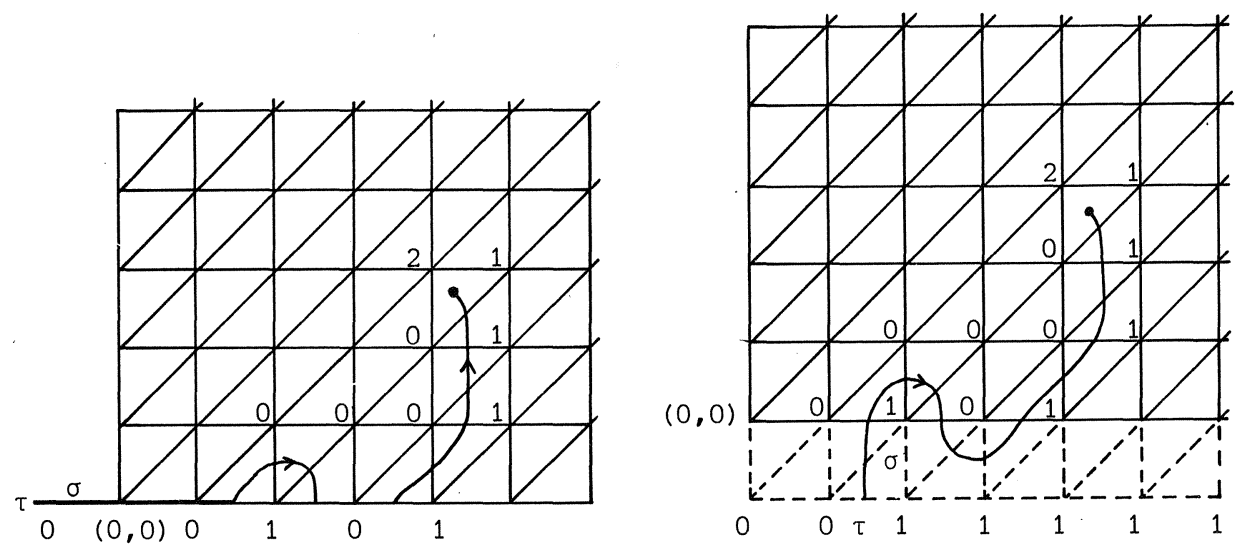

Figure 4.5.1.a $n=2, k^{*}=0 y=(0,0)^{\top}$. Figure 4.5.1.b $n=2, k^{*}=1, y=(2,0)^{\top}$.

Lüthi [1976] proposed an algorithm which is closely related to the case $\mathrm{k}^{*}=0$. This algorithm is based on the standard labelling rule on $\mathrm{R}^{\mathrm{n}}$. It can be viewed as a generalization of the case $k^{*}=0$ in the sense that the sequence of found labels is not predetermined. On the other hand it is a special case of the variable dimension restart algorithm to be presented in the chapters 5 and 6.

\subsection{SANDWICH METHOD.}

All the methods described in the previous sections suffer from inefficiency, since they are characterized by a start outside the region of interest and by a fixed grid throughout the algorithm. If we have a small grid size the number of iterations becomes very high whereas the approximation is bad if the grid size is large. 
A method which avoids this disadvantage is the Sandwich method introduced for functions on $S^{n}$ by Kuhn and Mackinnon [1975]. The method was discovered earlier by Merrill $[1971,1972]$ for mappings on unbounded regions.

The Sandwich method is a straightforward application of Kuhn's artificial start algorithm. Suppose we want to compute a fixed point of a continuous function $f$ from $S^{n}$ into itself. Therefore $S^{n}$ is embedded in the $(n+1)-$ dimensional simplex $\mathrm{s}^{\mathrm{n}+1}$, which is triangulated according to definition 3.4.1. Clearly $s_{n+2}^{n+1}$ corresponds to $s^{n}$ i.e. $x=\left(x_{1}, \ldots, x_{n+1}, 0\right)^{\top}$ is an element of $s_{n+2}^{n+1}$ iff $\left(x_{1}, \ldots, x_{n+1}\right)^{\top}$ is an element of $s^{n}$. Let $l$ denote the standard labelling rule on $s^{n}$ as defined in 4.2.1. Now the points of $s^{n+1}$ are labelled by $\bar{\ell}(\mathrm{x})$ defined as follows

$$
\begin{array}{ll}
\bar{l}(\mathrm{x})=\mathrm{n}+2 & \text { if } \mathrm{x}_{\mathrm{n}+2}>0 \\
\bar{\ell}(\mathrm{x})=\ell\left(\mathrm{x}_{1}, \ldots, \mathrm{x}_{\mathrm{n}+1}\right) & \text { if } \mathrm{x}_{\mathrm{n}+2}=0
\end{array}
$$

So all points with $x_{n+2}>0$ are labelled by $n+2$. Let $y$ be an arbitrarily chosen point of $s_{n+2}^{n+1}$. Note that in Kuhn's algorithm $y$ must be the barycenter of $s_{n+2}^{n+1}$ but that the extreme case $k^{*}=n$ of the class of algorithms discussed in section 4 allows for an arbitrarily chosen point $y$. Then starting with an $(n+1)$-simplex such that $y$ is one of its vertices and the convex hull of the other vertices is the unique $I_{n+1}$-complete simplex (see definition 4.4.2) of $\bar{s}_{n+2}^{n+1}$, the algorithm generates a path of adjacent simplices until a completely labelled $(n+1)$-simplex of $s^{n+1}$ is found. Clearly this is the case as soon as a grid point is generated such that $x_{n+2}>0$, i.e. if a simplex $\sigma\left(y^{1}, \pi\left(I_{n+1}\right)\right)$ is generated with $y_{n+2}^{1}=0$ and $\pi_{n+1}^{-}=n+1$. But then $y_{n+2}^{i}=0$ for $i=1, \ldots, n+1$ and $\tau\left(y^{1}, \ldots, y^{n+1}\right)$ is an $I_{n+1}$-complete facet of $\sigma$ lying in $s_{n+2}^{n+1}$ or analogously $\tau$ is a completely labelled simplex of $s^{n}$ and yields an approximation of a fixed point of $\mathrm{f}$. In figure 4.6 .1 the Sandwich method is illustrated for the example of figure 4.4 .1 by drawing the intersection of the path of simplices in $\bar{S}^{-n+1}$ with the $(n+2)$-th boundary $s_{n+2}^{n+1}$ of $s^{n+1}$. Observe that seven function evaluations are made, whereas also seven points of the artificial labelled boundary $\bar{s}_{n+2}^{n+1}$ are generated. The generalization to $\mathrm{R}_{+}^{\mathrm{n}}$ is immediately clear from the generalization of Kuhn's artificial start algorithm to $R_{+}^{n}$ as described in section 4.5 . The extension to $\mathrm{R}^{\mathrm{n}}$ can easily be made and will be discussed in section 10 for 
vector labelling in stead of integer labelling.

The advantage of this method is that $y$ can be chosen to be any grid point of $s_{n+2}^{n+1}$, or equivalently, of $s^{n}$. Therefore we may apply the algorithm for a

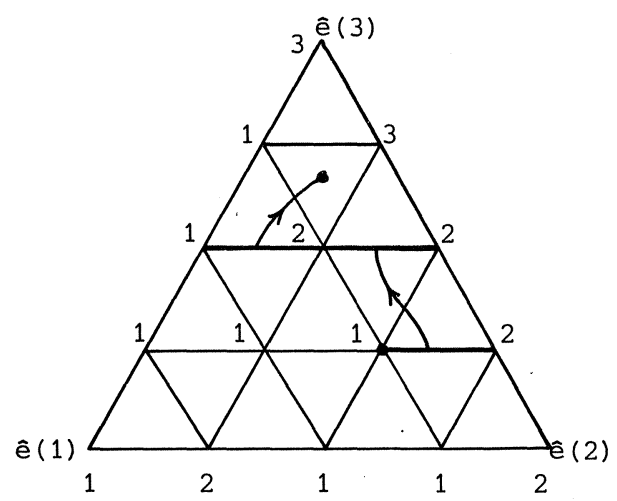

Figure 4.6.1. The intersection of the path of simplices, generated by the Sandwich method, with $s_{n+2}^{n+1}, y=\left(\frac{1}{4}, \frac{1}{2}, \frac{1}{4}\right)^{\top}, n=2$, ê $(i)=\left(e^{\top}(i), 0\right)^{\top}$.

decreasing sequence of grid sizes. In any stage the approximate solution of the previous stage can be used as the starting point. So, a sequence of approximations is generated, until the desired accuracy is achieved.

\subsection{HOMOTOPY ALGORITHM.}

A second algorithm which avoids the disadvantages of the methods discussed in the sections 3 until 5 was developed by Eaves [1972] for mappings on $\mathrm{S}^{\mathrm{n}}$ and by Eaves and Saigal [1972] for mapnings on $\mathrm{R}^{\mathrm{n}}$. To handle mappings, vector labelling has to be used. Then the method traces a path of fixed points of a homotopy function from a given linear function to the function of interest. Therefore the method was called the homotopy algorithm. We return to the matter of vector labelling in the following sections. In this section we discuss the method using integer labelling. The algorithm is characterized by an automatically decreasing grid size. We treat at first the simplest applicationwith the decreasing sequence $1,1 / 2,1 / 3, \ldots$ to compute a fixed point of a continuous function $f$ on $s^{n}$. Therefore $T^{n+1}$ is triangulated by the standard triangulation with grid size $\delta=1$ and with e(1) a grid point of the triangulation. Now, let 
$\mathrm{u}^{\mathrm{n}+1}$ be defined by

$$
U^{n+1}=\left\{x \in T^{n+1} \mid x_{1}, \ldots, x_{n+1} \geq 0 \text { and } x_{n+2} \leq 0\right\}
$$

and let $\mathrm{U}^{\mathrm{n}+1}(\mathrm{~m})$ be defined by

$$
\mathrm{U}^{\mathrm{n}+1}(\mathrm{~m})=\left\{\mathrm{x} \in \mathrm{U}^{\mathrm{n}+1} \mid \mathrm{x}_{\mathrm{n}+2}=-\mathrm{m}+1\right\} \quad \mathrm{m}=1,2, \ldots .
$$

Clearly the triangulation of $\mathrm{T}^{\mathrm{n}+1}$ induces a triangulation of $\mathrm{U}^{\mathrm{n}+1}$. Moreover, the induced triangulation of $\mathrm{U}^{\mathrm{n}+1}(\mathrm{~m})$ corresponds with the standard triangulation of $\mathrm{s}^{\mathrm{n}}$ with grid size $\mathrm{m}^{-1}$, i.e. the point $\left(x_{1}, \ldots, x_{n+1},-m+1\right)^{\top}$ of $u^{n+1}(m)$ corresponds with the point $m^{-1}\left(x_{1}, \ldots, x_{n+1}\right)^{\top}$ of $s^{n}$ in the sense that the latter point is the intersection of the line from $\left(x_{1}, \ldots, x_{n+1},-m+1\right)^{\top}$ to $(0, \ldots, 0,1)^{\top}$ with $U^{n+1}(1)$. Consequently, the point $\left(x_{1}, \ldots, x_{n+1}, x_{n+2}\right)$ of $U^{n+1}$ is labelled by the standard labelling rule $\ell(y)$ on $s^{n}$ where $y_{i}=x_{i} /\left(1-x_{n+2}\right), i=1, \ldots, n+1$. Obviously $\ell(x) \neq i$ if $x_{i}=0$ for any $x \in U^{n+1}$. Furthermore an $I_{n+1}$-complete simplex of $U^{n+1}(m)$ corresponds with a completely labelled simplex of the standard triangulation of $s^{\mathrm{n}}$ with grid size $\mathrm{m}^{-1}$.

Since $U^{n+1}$ (1) corresponds with $S^{n}$ it is $I_{n+1}$-complete. The algorithm starts with the simplex $\sigma^{0}\left(y^{1}, \pi\left(I_{n+1}\right)\right)$ of $U^{n+1}$ such that $y^{1}=(1,0, \ldots, 0,1,-1)^{\top}$, $\pi(1)=n+1$ and $\pi_{i}=i-1, i=2, \ldots, n+1$, i.e. $\sigma^{0}\left(y^{1}, \pi\left(I_{n+1}\right)\right)$ is the unique simplex of the triangulation of $U^{n+1}$ having $U^{n+1}(1)$ as a facet. According to the replacement steps of table 3.5.1 the algorithm generates a path of adjacent $(\mathrm{n}+1)$-simplices of $\mathrm{U}^{\mathrm{n}+1}$ having $\mathrm{I}_{\mathrm{n}+1}$-complete common facets. Clearly $U^{n+1}(1)$ is the only $I_{n}$-complete facet on the boundary of $U^{n+1}$. Therefore, since cycling cannot occur all steps are feasible and an $I_{n+1}$-complete simplex $\tau$ of $U^{n+1}(m) m=2,3, \ldots$ is generated within a finite number of steps. The method is illustrated in figure 4.7.1. Observe that it is possible that the algorithm returns to a previous level. The algorithm can be broken down if either an a priori chosen value of $\mathrm{m}$ is attained or the approximation is good enough. Observe that in the example the vertices of the facet $\tau$ between the levels 2 and 3 represent the same points as the vertices of facet $\rho$ on level 6 . Therefore triangulations which allow for a faster decreasing of the grid size are interesting. 


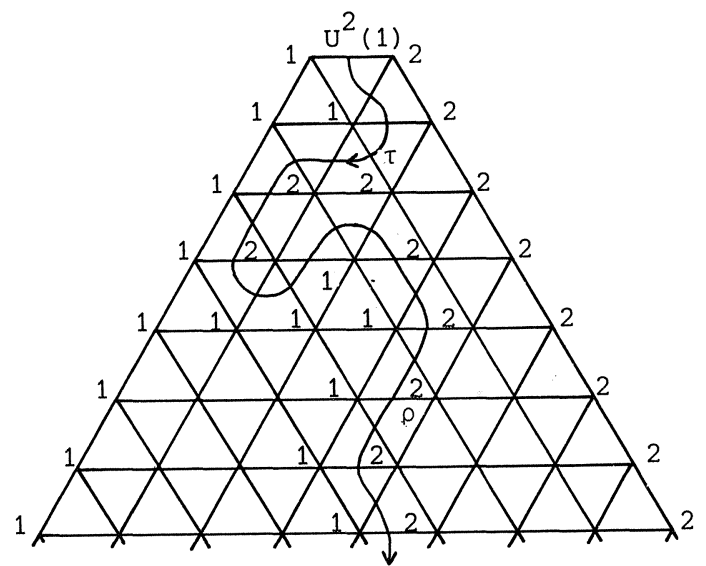

Figure 4.7.1. Illustration of the homotopy algorithm for $n=1$ with the decreasing sequence of grid sizes $1,1 / 2,1 / 3, \ldots$.

In Eaves [1972] a triangulation for a decreasing sequence of grid sizes with a factor of two was given. Let $G$ be the standard triangulation of the convex hull of $U^{n+1}(1)$ and $U^{n+1}(2)$ and let $G(k)$ be the standard triangulation of $\mathrm{U}^{\mathrm{n}+1}(\mathrm{~m})$, where $\mathrm{m}=2^{\mathrm{k}}$, for $\mathrm{k}=1,2, \ldots$ The grid size of $\mathrm{G}$ and $G(k) k=1,2, \ldots$ is one. Clearly, an $(n+1)$-simplex $\sigma$ of $G$ is characterized by $\sigma=\sigma\left(y^{1}, \pi\left(I_{n+1}\right)\right)$, whereas an $n$-simplex $\tau$ off $G(k)$ is characterized by $\tau=\tau\left(\mathrm{y}^{1}, \pi\left(I_{\mathrm{n}}\right)\right)$. We are now prepared to present a triangulation of $U^{\mathrm{n}+1}$ with a factor 2 between two subsequent levels. The $(n+1)$-simplices of $\mathrm{U}^{\mathrm{n}+1}$ are of the form $T_{\tau}(\sigma)$ with for some $n$-simplex $\tau$ of $G(k), k=1,2, \ldots$ and for some $(n+1)$-simplex $\sigma$ of $G, T_{\tau}(\sigma)$ defined by

$$
T_{\tau}(\sigma)=\bar{\sigma}\left(T_{\tau}\left(y^{1}\right), \ldots, T_{\tau}\left(y^{n+2}\right)\right)
$$

where $y^{1}, \ldots, y^{n+2}$ are the vertices of $\sigma$ and where for $j=1, \ldots, n+2$ the $h-t h$ component $t_{h}\left(y^{j}\right)$ of the vector $T_{\tau}\left(y^{j}\right)$ is equal to

$$
t_{h}\left(y^{j}\right)=\sum_{i=1}^{n+1} u_{h}^{i} y_{i}^{j} \quad h=1, \ldots, n+1
$$

and

$$
t_{n+2}\left(y^{j}\right)=1-\sum_{h=1}^{n+1} t_{h}\left(y^{j}\right)
$$

if $u^{1}, \ldots, u^{n+1}$ are the vertices of $\tau$. 


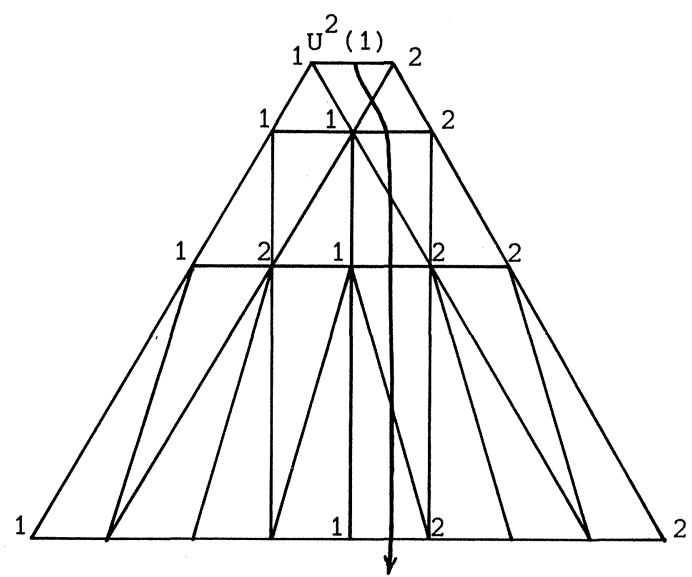

Figure 4.7.2. Illustration of the homotopy algorithm for $n=1$ with a factor of two between two subsequent levels.

In Eaves and Saigal [1972] the homotopy algorithm was generalized for the computation of a fixed point of a function on $R^{n}$ (if any). Let $G(0)$ be the standard triangulation of $\mathrm{R}^{\mathrm{n}} \times\{1\}$ with grid size $\delta$. Let $\tau$ be a simplex of $G(0)$. Then the triangulation of $\tau \times[1, \infty)$ is a scaled copy of the triangulation of $\mathrm{U}^{\mathrm{n}+1}$. In their paper Eaves and Saigal showed that these

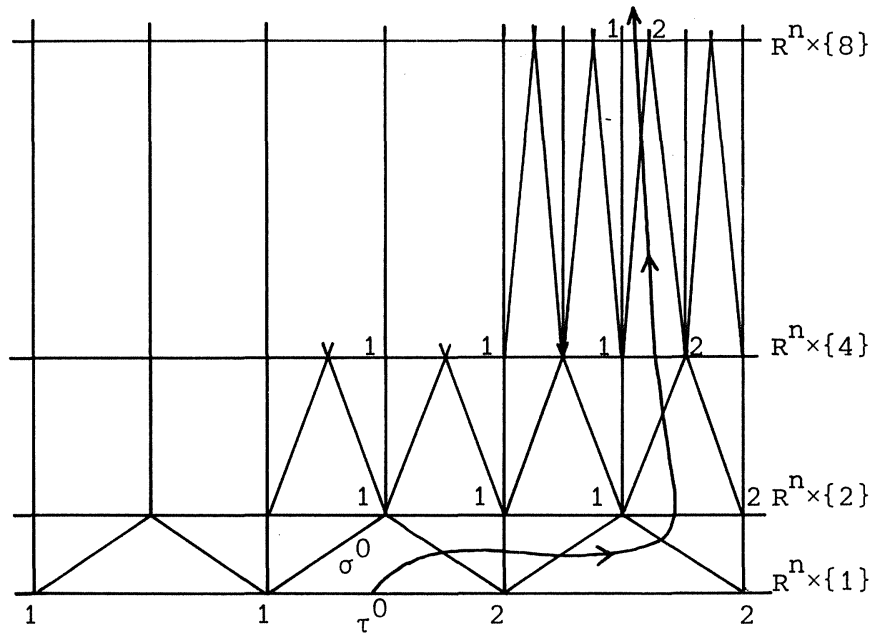

Figure 4.7.3. Illustration of the homotopy algorithm on $R^{n}$ for $n=1$. 
triangulations of $\tau \times[1, \infty)$ can be combined to a triangulation of $R^{\mathrm{n}} \times[1, \infty)$. Then the algorithm is started with a simplex $\sigma^{0}$ having some grid point $\mathrm{y}$ in $R^{n} \times\{2\}$ as a vertex while the facet $\tau^{0}$ opposite $y$ is a facet of $R^{n_{1}} \times\{1\}$. To guarantee feasibility of the replacement steps the points of $R^{n} \times\{1\}$ are artificially labelled in such a way that $\tau^{0}$ is the only completely labelled $n$-simplex of $R^{n} \times\{1\}$. Starting with $\sigma^{0}$ a path of adjacent $(n+1)$-simplices having common completely labelled facets is generated. An illustration for $\mathrm{n}=1$ is given in figure 4.7.3.

\subsection{VECTOR LABELLING AND APPROXIMATION.}

Assume we want to compute a fixed point of an upper-semicontinuous mapping from $R^{n}$ into itself. Therefore we introduce the concept of vector labelling.

Assume that $f$ is a piecewise linear approximation to $\phi$ with respect to a triangulation $G$ of $R^{n}$ (see definition 2.5.4). Then we define the following labelling rule.

DEFINITION 4.8.1. (Vector labelling on $\mathrm{R}^{\mathrm{n}}$ ) .

Let $b$ be a nonnegative vector in $R^{n+1}$ such that $b_{n+1}>0$. Then $x \in R^{n}$ receives the $(n+1)$-dimensional vector label $\ell(x)$ with

$$
\begin{aligned}
& \ell_{i}(x)=f_{i}(x)-x_{i}+b_{i} \quad i=1, \ldots, n, \\
& \ell_{n+1}(x)=b_{n+1} .
\end{aligned}
$$

For vector labelling we define a completely labelled simplex as follows.

DEFINITION 4.3.2. A simplex $\sigma\left(w^{1}, \ldots, w^{n+1}\right)$ of $G$ is completely labelled if the set of linear equations

$$
\sum_{i=1}^{n+1} \lambda_{i} l\left(w^{i}\right)=b
$$

has a nonnegative solution $\lambda^{*}=\left(\lambda_{1}^{*}, \ldots, \lambda_{n+1}^{*}\right)$. 
The following lemma states that a completely labelled simplex yields a fixed point of $\mathrm{f}$.

LEMMA 4.8.3. The piecewise linear approximation $f$ to $\phi$ has a fixed point in the simplex $\sigma\left(w^{1}, \ldots, w^{n+1}\right)$ if and only if $\sigma$ is completely labelled. If $\lambda^{*}$ is the solution of the system of linear equations, then

$$
x^{*}=\sum_{i=1}^{n+1} \lambda_{i}^{*} w^{i}
$$

is the fixed point of $f$ in $\sigma\left(w^{1}, \ldots, w^{n+1}\right)$.

PROOF. Clearly, since $\ell_{n+1}\left(w^{i}\right)=b_{n+1}$ for all $i$, we have that $\sum_{i=1}^{n+1} \lambda_{i}^{*}=1$. Moreover, by the definition of $f$ and $x$ * we have that

$$
\begin{aligned}
b_{j} & =\sum_{i=1}^{n+1} \lambda_{i}^{*} \ell_{j}\left(w^{i}\right)=\sum_{i=1}^{n+1} \lambda_{i}^{*} f_{j}\left(w^{i}\right)-\sum_{i=1}^{n+1} \lambda_{i}^{*} w_{j}^{i}+b_{j} \sum_{i=1}^{n+1} \lambda_{i}^{*}= \\
& =f_{j}\left(x^{*}\right)-x_{j}^{*}+b_{j} \quad j=1, \ldots, n,
\end{aligned}
$$

which proves that $x^{*}$ is a fixed point of $f$.

Conversely, if $x$ is a point of $\sigma\left(w^{1}, \ldots, w^{n+1}\right)$, then there is a $\lambda \in s^{n}$, such that $\mathrm{x}=\sum_{i=1}^{n+1} \lambda_{i} w^{i}$. So $\ell(\mathrm{x})=\ell\left(\sum_{i=1}^{n+1} \lambda_{i} w^{i}\right)=\sum_{j=1}^{n+1} \lambda_{i} \ell\left(w^{i}\right)$. If $x^{*}$ is a fixed point then $\ell_{j}\left(x^{*}\right)=f_{j}\left(x^{*}\right)-x_{j}^{*}+b_{j}=b_{j}$ for $j=1, \ldots, n$, which implies that

$$
\sum_{i=1}^{n+1} \lambda_{i}^{*} l\left(w^{i}\right)=b
$$

when $x^{*}=\sum_{i=1}^{n+1} \lambda_{i}^{*} w^{i}$. Hence $\sigma\left(w^{1}, \ldots, w^{n+1}\right)$ is completely labelled.

Let $\sigma^{*}$ be a completely labelled simplex and let $x^{*}$ be the fixed point of $f$ in $\sigma^{*}$. The next theorem shows the accuracy of $x^{*}$ as approximate fixed point of the mapping $\phi$.

THEOREM 4.8.4. Let $\varepsilon>0$ be such that for all $\mathrm{g}(\mathrm{y}) \epsilon \phi(\mathrm{y})$ there exists an element $g(x) \in \phi(x)$ such that $\max _{i \in I_{n}}\left|g_{i}(y)-g_{i}(x)\right|<\varepsilon$ for all $x, y \in \sigma^{*}$. Then there is an element $g\left(x^{*}\right) \in \phi\left(x^{*}\right)$ such that $\max _{i \in I_{n}}\left|g_{i}\left(x^{*}\right)-x_{i}^{*}\right|<\varepsilon$. 
PROOF. We have that $x^{*}=\sum_{i=1}^{n+1} \lambda_{i}^{*} w^{i}=\sum_{i=1}^{n+1} \lambda_{i}^{*} f\left(w^{i}\right)$. Since $w^{j} \in \sigma^{*}$ there exists an element $g\left(x^{*}\right) \in \phi\left(x^{*}\right)$ such that

$$
\max _{i \in I_{n}}\left|f_{i}\left(w^{j}\right)-g_{i}\left(x^{*}\right)\right|<\varepsilon, \quad j=1, \ldots, n+1 .
$$

Hence

$$
\begin{aligned}
\max _{i \in I_{n}}\left|g_{i}\left(x^{*}\right)-x_{i}^{*}\right| & =\max _{i \in I_{n}}\left|\sum_{j=1}^{n+1} \lambda_{j}^{*}\left\{g_{i}\left(x^{*}\right)-f_{i}\left(w^{j}\right)\right\}\right| \\
& \leq \sum_{j=1}^{n+1} \lambda_{j}^{*} \max _{i \in I_{n}}\left|g_{i}\left(x^{*}\right)-f_{i}\left(w^{j}\right)\right|<\varepsilon .
\end{aligned}
$$

COROLLARY 4.8.5. Let $\phi$ be a continuous function from $R^{n}$ into itself and let $\mathrm{x}^{*}$ be the fixed point of the piecewise linear approximation $f$ to $\phi$ in the comnletely labelled simplex $\sigma^{*}$. Then

$$
\max _{i \in I_{n}}\left|\phi_{i}\left(x^{*}\right)-x_{i}^{*}\right|<\varepsilon .
$$

Observe that for a continuous function $\phi$ from $R^{n}$ into itself $\max _{i}\left|\phi_{i}\left(\mathrm{x}^{*}\right)-\mathrm{x}_{i}^{*}\right|$ is bounded by $\varepsilon$ when vector labelling is used whereas this amount is bounded by $\varepsilon+\delta$ when using integer labelling (see lemma 4.2.4). Using vector labelling to compute a fixed point of a continuous function on $S^{n}$ the upper bound becomes $\varepsilon$ instead of $n(\varepsilon+\delta)$ in case of integer labelling (see lemma 4.2.2). The result of theorem 4.8 .4 while true, may not be at all useful. Of course, in case of a continuous function diam $\sigma^{*}$ close to zero implies that $\varepsilon$ becomes very small. However, it can easily be seen that there exist u.s.c. mappings such that $\varepsilon$ may be rather large, (e.g. as large as 1 ), even if diam $\sigma^{*}$ is arbitrarily small. Nevertheless, computing a fixed point of $\phi$, we have that a sequence of triangulations with mesh going to zero, the sequence of approximate fixed points contains a subsequence converging to a fixed point of $\phi$ (if any, see theorem $2.5 .3)$.

\subsection{A BASIC ALGORITHM TO COMPUTE A FIXED POINT OF A MAPPING.}

One of the first methods to find a completely labelled simplex using vector labelling was developed by Eaves [1971]. Let $\phi$ be an uppersemicontinuous mapping from an n-dimensional compact, convex subset $\mathrm{C}$ into 
itself and let $c$ be an element in the interior of $c$. The mapping $\phi$ is replaced by the u.s.c. mapping $\bar{\phi}$ from an $n$-simplex $C^{\prime}$ containing $C$ in its interior to $\mathrm{C}$ as in the proof of theorem 2.5 .3 , i.e.

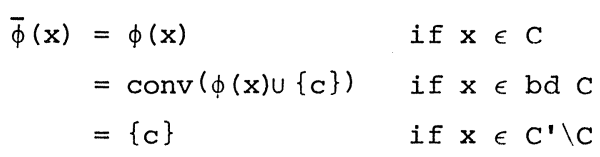

Recall that a fixed point of $\bar{\phi}$ is a fixed point of $\phi$.

Let $\mathrm{G}$ be a triangulation of $\mathrm{C}^{\prime}$ and let the starting point $\mathrm{x}^{0}$ be an arbitrarily chosen point on bd $C^{\prime}$, such that $\mathrm{x}^{0}$ is in the interior of $a$ facet $\tau^{0}$ of the triangulation G. Let $\sigma^{0}$ be the unique simplex of $G$ having $\tau^{0}$ as a facet (see theorem 2.3.5) and let $d$ be the $(n+1)$-dimensional vector $\left.\left(x^{0}-c\right)^{\top}, 0\right)^{\top}+b$.

Before describing the algorithm we introduce the concent of an almost complete simplex.

DEFINITION 4.9.1. For $k=n-1$ or $k=n$, a $k$-simplex $\sigma\left(w^{1}, \ldots, w^{k+1}\right)$ of $G$ is almost complete if the system of linear equations

$$
\sum_{i=1}^{k+1} \lambda_{i} \ell\left(w^{i}\right)+\mu d=b
$$

has a nonnegative solution $\left(\lambda_{1}^{*}, \ldots, \lambda_{k+1}^{*}, \mu^{*}\right)$.

Although degeneracy can occur in fixed point algorithms we assume in the following that it does not happen. A method to resolve the problem of degeneracy can be found in e.g. Todd [1976a]. S̀o assuming non degeneracy the system of linear equations has a unique solution if $\sigma$ is an almost complete (n-1)-simplex. However, if $\sigma$ is an almost complete $n$-simplex the above system of linear equations has two solutions having each just one variable equal to zero. Clearly, $\sigma$ is completely labelled if for one of these two solutions $\mu^{*}=0$. The algorithm searches for a simplex having such a solution.

Since $\tau^{0}$ is a boundary facet its vertices $y^{i} i \in I_{n}$ are labelled by $\ell\left(y^{i}\right)=$ $\left.\left(c-y^{i}\right)^{\top}, 0\right)^{\top}+b$. Clearly, $x^{0}=\sum_{i=1}^{n} \alpha_{i} y^{i}$ for some $\alpha \in S^{n-1}$. Since $x^{0}$ is in the interior of $\tau^{0}, \alpha_{i}>0 i \in I_{n}$. So, the (n-1)simplex $\tau^{0}$ is almost complete with solution $\left(\lambda_{i}^{*}, \ldots, \lambda_{n}^{*} \mu^{*}\right)$ where $\lambda_{i}^{*}=\frac{1}{2} \alpha_{i} i \in I_{n}$ and $\mu^{*}=\frac{1}{2}$. Moreover, $\tau^{0}$ is the only almost complete $(n-1)$-simplex on the boundary of $C^{\prime}$ 
(see Eaves [1971] or Todd [1976a]). Now the algorithm is described by the following steps where $\sigma^{0}=\sigma\left(y^{1}, \ldots, y^{n+1}\right)$ is the unique simplex containing $\tau^{0}$.

Step 0 . Set $\sigma$ equal to $\sigma\left(y^{1}, \ldots, y^{n+1}\right)$ and let $\bar{y}=y^{t}$ be the vertex of $\sigma^{0}$ not in $\tau^{0}$. Let $\Sigma_{i \neq t} \lambda_{i} l\left(y^{i}\right)+\mu d=b$ be the set of linear equations induced by $\tau^{0}$. This system has the above mentioned solution $\left(\lambda_{1}^{*}, \ldots, \lambda_{n^{*}}^{*}{ }^{*}\right)$.

Step 1. Calculate $\ell(\bar{y})$ and make a standard linear programming pivot step by bringing $l(\bar{y})$ in the system of linear equations to obtain a new solution with one of variables equal to zero. If $\mu$ becomes zero, i.e. if d is eliminated, $\sigma$ is a completely labelled simplex and the algorithm terminates. Otherwise, for some $s, l\left(y^{\mathbf{s}}\right)$ is eliminated by the pivot step.

Step 2. Adapt $\sigma$ by replacing $y^{\mathbf{S}}$. Let $\bar{y}$ be the new vertex of $\sigma$ and return to step 1 .

Since non degeneracy is assumed, all the replacement steps are unique, so cycling cannot occur (see section 4). Moreover, all replacement steps are feasible since $\tau^{0}$ is the only almost complete facet on the boundary of $C^{\prime}$. Hence the algorithm terminates within a finite number of iterations with a completely labelled simplex. Note that the algorithm has the same disadvantages of those discussed in the sections 3 until 5. It must start outside $\mathrm{C}$ and the grid size is kept fixed.

\subsection{SANDWICH AND HOMOTOPY METHODS ON $R^{n}$.}

Let $\phi$ be an upper semi-continuous mapping from $\mathrm{R}^{\mathrm{n}}$ into itself and suppose we want to compute a fixed point (if any). As remarked in section 6 the Sandwich method is the application of Kuhn's artificial start algorithm on $R^{n \times R_{+}}$by adding an extra layer of points with $x_{n+1}=-1$. From the discussion in 6 it is clear that the algorithm terminates as soon as a point is generated with $x_{n+1}=1$. This means that in practice only simplices $R^{n} \times[-1,0]$ are generated, i.e. the algorithm starts with the $(n+1)$-simplex having the unique completely labelled $n$-simplex in $R^{n} \times\{-1\}$ of artificially labelled points as a facet and terminates with an $(n+1)-$ simplex having a completely labelled facet in $R^{n} \times\{0\}$, whose vertices are labelled according to the standard labelling rule induced by the mapping $\phi$. Therefore we can restri.ct ourselves to the set $R^{n} \times[-1,0]$ or 
equivalently to $R^{n} \times[0,1]$, where $R^{n_{x}}\{0\}$ will be the level of artificially labelled points.

To apply the Sandwich method we define the mapping $\bar{\phi}$ from $R^{n} \times[0,1]$ to $R^{n}$ by

$$
\bar{\phi}(x, t)=\{t y+(1-t) v \mid y \in \phi(x)\},
$$

where $\mathrm{v} \in \mathrm{R}^{\mathrm{n}}$ is an arbitrarily chosen point, called the starting point. Now, a point $\left(x^{*}, t^{*}\right)$ is called a fixed point of $\bar{\phi}$ if $x^{*} \in \bar{\phi}\left(x^{*}, t^{*}\right)$. Clearly, if $\left(x^{*}, 1\right)$ is a fixed point of $\bar{\phi}$, then $x^{*}$ is a fixed point of $\phi$. Furthermore, it is obvious that $(v, 0)$ is the only fixed point of $\bar{\phi}$ in $R^{n} \times\{0\}$. Let $G$ be the triangulation of $R^{n} \times[0,1]$ induced by the $A K$ triangulation of $\mathrm{R}^{\mathrm{n}+1}$, where $\mathrm{A}$ is the diagonal matrix such that $a_{i i}=\delta, i=1, \ldots, \mathrm{n}$ and $a_{i i}=1$ for $i=n+1$, i.e. the grid points $G$ are either in $R^{n} \times\{0\}$ or $R^{n} \times\{1\}$ and the induced triangulations of $R^{n} \times\{0\}$ and $R^{n} \times\{1\}$ have grid size $\delta$. We assume that $(v, 0)$ is in the interior of an $n$-simplex $\tau^{0}$ of $R^{n} \times\{0\}$. A point $(x, t)$ receives the vector label $\ell(x)$ defined in 4.3 .1 , i.e. $l_{i}(x, t)=f_{i}(x, t)-x_{i}+b_{i}, i=1, \ldots, n$ and $l_{n+1}(x, t)=b_{n+1}$ with $f(x, t) a$ piecewise linear approximation to $\bar{\phi}$ with respect to $G$. Since $(v, 0)$ is the only fixed point with $t=0$ it follows from lemma 4.8 .3 that $\tau^{0}$ is the on? $y$ completely labelled $n$-simplex in $R^{n} \times\{0\}$.

The algorithm proceeds as follows.

Step 0 . Set $\sigma$ equal to the unique $(n+1)$-simplex having $\tau^{0}$ as a facet and let $\bar{y}$ be the vertex of $\sigma$ not in $\tau^{0}$. Let $\Sigma_{i=1}^{n+1} \lambda_{i} l\left(y^{i}\right)=b$ be the system of linear equations induced by $\tau^{0}$ where $y^{1}, \ldots, y^{n+1}$ are the vertices of $\tau^{0}$. The solution of this system is $\lambda^{*}=\left(\lambda_{1}^{*}, \ldots, \lambda_{n+1}^{*}\right)$ if $\mathrm{v}=\sum_{i=1}^{\mathrm{n}+1} \lambda_{i}^{*} \mathrm{y}^{i}$.

Step 1. Calculate $\ell(\bar{y})$. Make a linear programing step with $\ell(\bar{y})$ in the system of linear equations. Let $s$ be the index such that $\ell\left(y^{s}\right)$ is eliminated by $\ell(\bar{y})$.

Step 2. If the facet of $\sigma$ opposite $y^{s}$ lies in $R^{n} \times\{1\}$, this facet is a completely labelled $n$-simplex of $R^{n} \times\{1\}$ and the algorithm terminates. Otherwise, adapt $\sigma$ by replacing $\mathrm{y}^{\mathrm{S}}$ and return to step 1 with $\bar{Y}$ equal to the new vertex.

Following these steps, the algorithm generates a nath of adjacent simplices such that the common facets are completely labelled. It means, that the algorithm can be seen as a method which traces a path of fixed 
points of the piecewise linear approximation $f(x, t)$ to $\bar{\phi}(x, t)$. Using the standard arguments the algorithm either terminates within a finite number of steps or generates a path of simplices going to infinity. It can be proved that the algorithm terminates if Merrill's condition (see lemma 2.6.2) is satisfied (c.f. Merrill [1972] and also Todd [1976a]). The approximation of the fixed noint can be chosen as the starting point $v$ in a new application of the method with a smaller grid size.

To apply the homotopy algorithm we define a function $\tilde{\phi}$ from $R^{n} \times[1, \infty)$ to $R^{n}$ such that

$$
\tilde{\phi}(x, 1)=v \text { and } \tilde{\phi}\left(x, 2^{k}\right)=f^{k}(x)
$$

where $v$ is the starting point and $f^{k}(x)$ a piecewise linear approximation to $\phi$ with respect to the triangulation of $R^{n} \times\left\{2^{k}\right\} . R^{n} \times[1, \infty)$ is triangulated as in section 7 such that $v$ is in the interior of an $n$-simplex $\tau^{0}$ in $R^{n} \times\{1\}$. The points $(x, t)$ are labelled as above with $f(x, t)$ a piecewise linear approximation to $\tilde{\phi}(x, t)$. Hence $\tau^{0}$ is the only completely labelled $n$-simplex in $\mathbb{R}^{n} \times\{1\}$. Starting with the $(n+1)$-simplex having $\tau^{0}$ as a facet the algorithm generates a sequence of adjacent simplices such that the common facets are completely labelled. That means that a path of fixed points of the piecewise linear approximation $f(x, t)$ is traced.

It can easily be seen that this path converges to a fixed point of $\phi(x)$ if $t$ goes to infinity. The algorithm can be terminated as soon as a completely labelled simplex on a predetermined level $t$ is generated. This occurs in a finite number of steps if Merrill's condition is satisfied.

4.11. MAPPINGS FROM $\mathrm{S}^{\mathrm{n}}$ INTO $\mathrm{S}^{\mathrm{n}}$.

To compute a fixed point of an u.s.c. mapping $\phi$ on $S^{n}$ we define the following labelling rule given a triangulation $G$ of $s^{n}$.

DEFINITION 4.11.1. (Vector labelling on $s^{n}$ ).

$A$ point $x$ in $S^{n}$ receives the $(n+1)$-dimensional vector label $\ell(x)$ defined by

$$
\ell(x)=-f(x)+x+b
$$


where $f(x)$ is a piecewise linear approximation to $\phi$ with respect to $G$ and where $\mathrm{b}$ is a nonnegative vector with at least one component nositive. An $n$-simplex $\sigma\left(w^{1}, \ldots, w^{n+1}\right)$ of $G$ is called completely labelled if the system of linear equations

$$
\sum_{i=1}^{n+1} \lambda_{i} l\left(w^{i}\right)=b
$$

has a nonnegative solution $\lambda^{*}=\left(\lambda_{1}^{*}, \ldots, \lambda_{n+1}^{*}\right)$.

LEMMA 4.11.2. The point $\mathrm{x}^{*}=\sum_{i=1}^{\mathrm{n}+1} \lambda_{i}^{*}{ }^{\mathrm{w}}{ }^{i}$ is a fixed point of $f$ j.f and only if $\sigma\left(w^{1}, \ldots, w^{n+1}\right)$ is completely labelled with $\lambda^{*}=\left(\lambda_{1}^{*}, \ldots, \lambda_{n+1}^{*}\right)$ the solution of the system of linear equations.

PROOF. The proof of the lemma is analogous to the proof of lemma 4.8.3. Therefore we only show that $\sum_{i=1}^{n+1} \lambda_{i}^{*}=1$ if $\lambda^{*}$ is the solution of the system of linear equations. By summing up over all equations we have that

$$
\sum_{i=1}^{n+1} \lambda_{i}^{*} \sum_{j=1}^{n+1}\left(-f_{j}\left(w^{i}\right)+w_{j}^{i}+b_{j}\right)=\sum_{j=1}^{n+1} b_{j} .
$$

Hence $\Sigma_{i=1}^{n+1} \lambda_{i}^{*}=1$ since $\sum_{j=1}^{n+1} f_{j}\left(w^{i}\right)=\Sigma_{j=1}^{n+1} w_{j}^{i}=1$ for all $i$.

Analogous to the proof of theorem 4.8.4 the next corollary follows immediately.

COROLLARY 4.11.3. Let mesh $\mathrm{G}<\delta$ and let the mapping $\phi$ be such that $\phi(y) \epsilon \phi(x)+B^{n}(0, \varepsilon)$ if $y \in B^{n}(x, \delta)$. Then $x^{*} \epsilon \phi\left(x^{*}\right)+B^{n}(0, \varepsilon)$.

To approximate a fixed point of a mapping on $s^{\mathrm{n}}$ we can use the Sandwich method with a decreasing sequence of grid sizes and restarting in any stage with the approximation of the previous stage or the homotopy method involving an automatically decreasing grid size.

The application of the Sandwich method can be done as follows. As discussed in section $6, \mathrm{~S}^{\mathrm{n}+1}$ is triangulated in the standard way with grid size $\mathrm{m}^{-1}$. Clearly, only simplices are generated in the convex hull of $s_{n+2}^{n+1}$ and $s_{n+2}^{n+1}$. Therefore we consider the corresponding set $s^{n} \times[0,1]$, where $s^{n} \times\{0\}$ is triangulated with grid size $(n+1)^{-1}$, whereas $S^{n} \times\{1\}$ is triangulated with grid size $\mathrm{m}^{-1}$. Now we define the mapping $\bar{\phi}(\mathrm{x}, \mathrm{t})$ by 


$$
\bar{\phi}(x, t)=t \phi(x)+(1-t)\{v\}
$$

where $v$ is an arbitrarily chosen starting point such that $(v, 0)$ is in the interior of an n-simplex of the triangulation of $s^{n} \times\{0\}$. Clearly $(v, 0)$ is a fixed point of $\bar{\phi}(x, t)$. Again a path of fixed points of the piecewise linear approximation $f(x, t)$ to $\bar{\phi}(x, t)$ is traced, starting with $(v, 0)$ and terminating with a point on level one. To apply the homotopy method we triangulate the set $S^{\mathrm{n}} \times[1, \infty)$ in stead of $\mathrm{U}^{\mathrm{n}+1}$. This set is triangulated by $(\mathrm{n}+1)$-simplices such that $\mathrm{s}^{\mathrm{n}} \times\left\{2^{\mathrm{m}}\right\}$ is triangulated by the standard triangulation with grid size $2^{-m}$. A path of fixed points of the piecewise linear approximation to $\tilde{\phi}(x, t)$ is traced with $\tilde{\phi}(x, t)$ a correspondence from $s^{n} x\{t\}$ to $s^{n}$ such that $\tilde{\phi}\left(x, 2^{m}\right)=f^{m}(x)$ where $f^{m}(x)$ is a piecewise linear approximation to $\phi(x)$ with respect to the triangulation of $\mathrm{s}^{\mathrm{n}} \times\left\{2^{\mathrm{m}}\right\} \quad \mathrm{m}=0,1, \ldots$.

\subsection{CONCLUDING REMARKS.}

All algorithms discussed in this chapter are based on the principle of generating a sequence of adjacent simplices (or primitive sets) until a completely labelled simplex yielding an approximation of a fixed point is found. An algorithm based upon the approximation of a curve by means of numerical solution of an initial value problem was introduced by Kellogg, Li and Yorke [1976] and has since been superceded by the so called continuation methods (see Allgower and Georg [1980]). The most sophisticated simplicial algorithms are the "Sandwich" method and the homotopy algorithm. In both methods the n-dimensional problem is embedded in an $(n+1)-$ dimensional one. The first one has the disadvantage that a layer of artificially labelled points is needed. So with vector labelling a path of fixed points is traced starting with a fixed point of a constant function at each restart. This can be improved by using the approximate Jacobian (see e.g. Todd [1978a]). In the homotopy method the mapping is continuously deformed. Moreover if corrections have to be made, e.g. if on a previous level a completely labelled simplex has been found which corresponds to a point which is nearly but not actually fixed, the simplices generated can move from fine triangulations back to coarser ones. On the other hand the grid size is reduced by a factor of at most two, whereas for the Sandwich method this factor can be chosen arbitrarily. So, in the latter method it 
is possible to accelerate the convergence by taking an increasing sequence of factors with which the grid size is reduced. In chapter 8 we present a homotopy algorithm which allows for an arbitrary factor between two subsequent levels. 
CHAPTER 5

VARIABLE DINENSION RESTART ALGORITHM

\subsection{INTRODUCTION.}

In the previous chapter several algorithms to compute a fixed point of a function or mapping from $s^{\mathrm{n}}$ into itself were discussed. Two efficient methods are the restart algorithm of Merrill and Kuhn and Mackinnon, which is referred to as the "Sandwich" method, and the continuous deformation algorithm of Eaves (and Saigal), called the homotopy method. In both methods a path of $(n+1)$-dimensional simplices is generated.

In this chapter a restart algorithm is presented which does not use a layer of artificially labelled points (see also Van der Laan and Talman [1979a,1980c]. Moreover it differs from the algorithms mentioned above by the fact that it does not generate a path of $(n+1)$-dimensional simplices but a path of $t$-simplices, where $t$ varies between 0 and $n$. More precisely, the method starts with a zero-dimensional simplex, called the starting point, generates a path of adjacent simplices of variable dimension and terminates with an $n$-dimensional simplex yielding an approximation of a fixed point. This algorithm will be referred to as the "variable dimension restart" algorithm, since the starting point can be arbitrarily chosen. Therefore the method can be applied for a decreasing sequence of grid sizes using. at any stage the approximation obtained in the previous stage as the starting point.

In section 2 a full description of the steps of the algorithm for integer labelling is given. A geometric interpretation of the method is discussed in section 3 and its convergence is proved in section 4. In section 5 the algorithm is put in the framework of the above mentioned methods by adding n+1 differently labelled points on an extra level. The application of the algorithm for vector labelling is discussed in section 6 and the 
generalization for computing a fixed point of a mapping on $\mathrm{T}^{\mathrm{n}}$ is treated in section 7. In particular we give some convergence conditions in this section. Numerical results are given in section 8 .

\subsection{DESCRIPTION OF THE STEPS OF THE ALGORITHM.}

Assume that we want to approximate a fixed point of a continuous function $f$ from $s^{n}$ into itself. Every point of $s^{n}$ receives an integer label based on the standard labelling rule on $s^{\mathrm{n}}$ as given in definition 4.2.1. Let $\mathrm{s}^{\mathrm{n}}$ be triangulated in the standard way with grid size $\mathrm{m}^{-1}$ (see definition 3.4.1). The amendments required for the Tr triangulation are straightforward. In definition 3.4 .1 the $(n+1) \times n$ matrix $Q$ is introduced to describe the simplices of the standard triangulation of $s^{n}$, whereas the simplices generated by the algorithms of chapter 4 were of the form $\sigma\left(\mathrm{y}^{1}, \pi\left(I_{\mathrm{k}}\right)\right)$. It will appear that the $(\mathrm{n}+1) \times \mathrm{n}$ matrix $Q$ is not sufficient to describe the simplices generated by the variable dimension restart algorithm. Therefore the matrix $Q$ is redefined as the $(n+1) \times(n+1)$ matrix

$$
\Omega=\left[\begin{array}{rrrrrrr}
-1 & 0 & \cdot & \cdot & \cdot & 0 & 1 \\
1 & \cdot & & & & 0 \\
0 & \cdot & & & & \cdot \\
\cdot & & \cdot & \cdot & & \cdot \\
\cdot & & & \cdot & -1 & \cdot \\
0 & \cdot & . & \cdot & 0 & 1 & -1
\end{array}\right] .
$$

So the matrix is extended with the column $q(n+1)=-\sum_{j=1}^{n} q(j)=(1,0, \ldots, 0,-1)^{\top}$. Now each column $q(i)$ of the matrix $Q$ can be interpreted as corresponding with the $i$-th direction, $i=1, \ldots, n+1$. This means that a search in the $i-t h$ direction involves a decrease of the $i$-th component and an increase of the $(i+1)-$ th component with $i+1=1$ when $i=n+1$. The algorithm to be described below builds up the set of labels consecutively. If $T \subset I_{n+1}$ is the set of labels already found a search is made in the directions $i, i \in T$ to find the other labels. Recall that by the proper labelling $l(x) \neq i$ if $x_{i}=0$. We define now a t-dimensional simplex $\sigma\left(\mathrm{Y}^{1}, \pi(T)\right)$. 
DEFINITION 5.2.1. For $t \leq n$, the $t$-dimensional simplex $\sigma\left(y^{1}, \pi(T)\right.$ ) of the standard triangulation of $\mathrm{s}^{\mathrm{n}}$ is the simplex with vertices $\mathrm{y}^{1}, \ldots, \mathrm{y}^{\mathrm{t+1}}$ such that
a) $\mathrm{y}^{1}$ is a grid point of the triangulation
b) $\pi(T)=\left(\pi_{1}, \ldots, \pi_{t}\right)$ is a permutation of the elements of $T$, $(|T|=t)$
c) $y^{i+1}=y^{i}+q\left(\pi_{i}\right) / m \quad i=1, \ldots, t$.

Observe that any $t$-dimensional simplex $\sigma\left(y^{1}, \pi(T)\right)$ has a unique representation when $t \leq n-1$, whereas any $n$-simplex has $n+1$ representations. Let $\pi(T)$ be a permutation of $n$ elements of the set $I_{n+1}$. Then $\sigma\left(y^{1}, \pi(T)\right)=\sigma\left(y^{i}, \pi^{i}\left(T^{i}\right)\right)$, where $T^{i}=I_{n+1} \backslash\left\{\pi_{i-1}\right\}$ and $\pi^{i}\left(T^{i}\right)=$ $=\left(\pi_{i}, \ldots, \pi_{n}, h, \pi_{1}, \ldots, \pi_{i-2}\right), i=2, \ldots, n+1$, wi.th $h$ the unique element of $I_{n+1}$ not in $T$.

We give now a generalization of definition 4.4.2

DEFINITION 5.2.2. A ( $t-1)$-simplex $\sigma, 1 \leq t \leq n+1$, is called $T$-complete if the $t$ vertices of $\sigma$ carry all the labels of the set $T(|\cdot|=t)$.

Note that $T=I_{n+1}$ if $t=n+1$ and that any zero-dimensional simplex $\{w\}$ is $\{l(w)\}$-complete. We are now ready to describe the steps of the algorithm. In this description the $(n+1)$-dimensional vector $R$ represents the "distance" between the starting point $\mathrm{v}$ and the vertex $\mathrm{y}^{1}$ of the last generated simplex $\sigma\left(y^{1}, \ldots, y^{t+1}\right)$, i.e. $y^{1}=v+\Sigma_{j=1}^{n+1} R_{j} q(j) / m$. The algorithm starts with an arbitrary chosen grid point $v$ and proceeds as follows.

Step 0. Set $t=0, y^{1}=v, T=\not, \sigma=\sigma^{0}\left(y^{1}, \pi(\emptyset)\right), \bar{y}=y^{1}$ and $R_{j}=0, j=1, \ldots, n+1$. Step 1. Calculate $\ell(\bar{y})$. If $\ell(\bar{y})$ is not an element of $T$ go to step 3 . Otherwise, there is exactly one vertex $y^{\mathbf{s}} \neq \overline{\mathrm{y}}$ such that $\ell\left(\mathrm{y}^{\mathbf{s}}\right)=$ $\ell(\bar{y})$.

Step 2. If $s=t+1$ and $R_{\pi_{t}}=0$ go to step 4. Otherwise $\sigma\left(y^{1}, \pi(T)\right)$ and $R$ are adapted according to table 5.2 .1 by replacing $\mathrm{y}^{\mathbf{S}}$. Return to step 1 with $\bar{y}$ equal to the new vertex of $\sigma$. 
Step 3. If $t=n$, a completely labelled simplex is found and the algorithm terminates. If $t<n, a(T \cup\{\ell(\bar{y})\})$-complete simnlex is found and the dimension is increased. $T$ becomes $T u\{\ell(\bar{y})\}, \pi(T)$ becomes $\left(\pi_{1}, \ldots, \pi_{t}, l(\bar{y})\right), \sigma$ becomes $\sigma\left(y^{1}, \pi(T)\right)$. Then $t$ becomes $t+1$ and return to step 1 with $\bar{y}=y^{t+1}$.

Step 4. The dimension is decreased by deleting $y^{\mathbf{s}}$. For exactly one index $s^{\prime}, s^{\prime} \leq t$ we have that $y^{s^{\prime}}$ is the vertex of $\sigma$ with label $\pi_{t}$. Now $T$ becomes $T \backslash\left\{\pi_{t}\right\}, \pi(T)$ becomes $\left(\pi_{1}, \ldots, \pi_{t-1}\right)$, $\sigma$ becomes $\sigma\left(\underline{y}^{1}, \pi(T)\right)$. Then $t$ becomes $t-1$ and return to step 2 with $s=s^{\prime}$.

Table 5.2.1. $\mathrm{s}$ is the index of the vertex to be replaced.

\begin{tabular}{l|l|l|l} 
& $y^{1}$ becomes & $\pi(T)$ becomes & $R$ becomes \\
\hline$s=1$ & $y^{1}+q\left(\pi_{1}\right) / m$ & $\left(\pi_{2}, \ldots, \pi_{t}, \pi_{1}\right)$ & $R+e\left(\pi_{1}\right)$ \\
$2 \leq s \leq t$ & $y^{1}$ & $\left(\pi_{1}, \ldots, \pi_{s-2}, \pi_{s}, \pi_{s-1}, \pi_{s+1}, \ldots, \pi_{t}\right)$ & $R$ \\
$s=t+1$ & $y^{1}-q\left(\pi_{t}\right) / m$ & $\left(\pi_{t}, \pi_{1}, \ldots, \pi_{t-1}\right)$ & $R-e\left(\pi_{t}\right)$
\end{tabular}

The algorithm generates a path of adjacent simplices starting with the zero-dimensional simplex $\{\mathrm{v}\}$. First a search is made in the $\ell(v)-t h$ direction to find the missing labels, i.e. the $\ell(v)-$ th component of $v$ is decreased whereas the $(\ell(v)+1)-$ th component is increased where $\ell(v)+1=1$ if $\ell(v)=n+1$. So one-dimensional simplices $\sigma\left(y^{1}, y^{2}\right)$ are generated with $\mathrm{y}^{1}=\mathrm{v}^{+} \mathrm{R}_{\ell(\mathrm{v})} \mathrm{q}(\ell(\mathrm{v})) / \mathrm{m}$ and $\mathrm{y}^{2}=\mathrm{y}^{1}+\mathrm{q}(\ell(\mathrm{v})) / \mathrm{m}$, such that the common zerodimensional facet is $\{l(v)\}$-complete. The algorithm proceeds with twodimensional simplices as soon as a second label is found, which must occur since $\ell(x) \neq i$ if $x_{i}=0$. In general if in step 1 a new label is found, say label $k$, we have for certain $t$, subset $T$ of ${ }^{-} I_{n+1}$, permutation $\pi(T)$, simplex $\sigma\left(y^{1}, \ldots, y^{t+1}\right)$ and vector $R$ generated by the algorithm that
a) $y^{1}=v+\sum_{j=1}^{n+1} R_{j} q(j) / m$
b) $R_{j}=0$ for $j \notin T$ and $R_{j} \geq 0$ for $j \in T$
c) $y^{i+1}=y^{i}+q\left(\pi_{i}\right) / m, \quad i=1, \ldots, t$
d) $\sigma$ is $(T u\{k\})$-complete.

Then the algorithm terminates with the completely labelled simplex $\sigma\left(y^{1}, \ldots, y^{n+1}\right)$ if $t=n$. Otherwise, the dimension is increased by setting $T=T u\{k\}$ and $t=t+1$, and a search is made with the labels $i, i \in T$ in all the 
directions $i, i \in T$, to find the other labels by generating a path of adjacent simplices $\sigma\left(y^{1}, \pi(T)\right)$ such that the common facets are T-complete. Doing so we must have that within a finite number of stens either again a new label is found or in step $2, s=t+1$ and $R_{\pi_{t}}=0$. In the latter case, by performing a replacement step according to table 5.2.1, $\mathrm{R}_{\pi}$ becomes negative indicating that the method searches in the direction $-\left(\pi_{t}\right)$ whereas label $\pi_{t}$ already has been found. To avoid this situation the dimension is decreased by setting $T=T \backslash\left\{\pi_{t}\right\}$ and $t=t-1$. The last vertex is deleted and the vertex with label $\pi_{t}$ is removed. In the first case the algorithm terminates or the dimension is again increased. So, the algorithm generates a path of adjacent simplices $\sigma^{0}, \sigma^{1}, \ldots$ of variable dimension. In section 4 we prove that a completely labelled simplex is found within a finite number of steps. Clearly, using this approximation the algorithm can be restarted with a finer grid, until a given accuracy is obtained. In figure 5.2.1 the method is illustrated for the example of figure 2.4.2. The results of the computation of the labels are indicated in the figure.

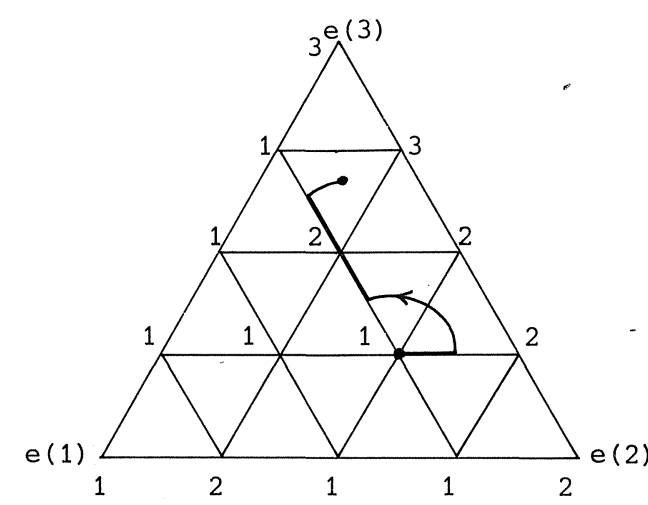

Figure 5.2.1. Illustration of the variable dimension restart method; the starting point $\mathrm{v}=\left(\frac{1}{4}, \frac{1}{2}, \frac{1}{4}\right)^{\top}, \mathrm{n}=2, \mathrm{~m}=4$.

In this example the algorithm performs the following steps, where $\sigma^{0}, \sigma^{1}, \ldots, \sigma^{6}$ is the sequence of generated simplices. 
1. Step 0: $t=0, u^{1}=v=\left(\frac{1}{4}, \frac{1}{2}, \frac{1}{4}\right)^{\top}, T=\varnothing, \sigma\left(y^{1}, \pi(T)\right)=\sigma^{0}=\sigma\left(u^{1}\right)$ and $R=(0,0,0)^{\top}$.

2. Step 1: $\ell\left(u^{1}\right)=1 \notin T$.

3. Step 3: $T=\{1\}, \pi(T)=(1), \sigma\left(y^{1}, \pi(T)\right)=\sigma^{1}=\sigma\left(u^{1}, u^{2}\right)$ with the new vertex $u^{2}=u^{1}+q(1) / 4=\left(0, \frac{3}{4}, \frac{1}{4}\right)^{\top}, t=1$.

4. Step 1: $\ell\left(u^{2}\right)=2 \notin T$.

5. Step 3: $T=\{1,2\}, \pi(T)=(1,2), \sigma\left(y^{1}, \pi(T)\right)=\sigma^{2}=\sigma\left(u^{1}, u^{2}, u^{3}\right)$ with the new vertex $u^{3}=u^{2}+q(2) / 4=\left(0, \frac{1}{2}, \frac{1}{2}\right)^{\top}, t=2$.

6. Step 1: $\ell\left(u^{3}\right)=2 \epsilon T, \ell\left(u^{3}\right)=\ell\left(u^{2}\right), u^{2}=y^{2}$, i.e. $s=2$.

7. Step 2: the vertex $u^{2}$ is replaced, $\pi(T)=(2,1), \sigma\left(y^{1}, \pi(T)\right)=\sigma^{3}=$ $=\sigma\left(u^{1}, u^{4}, u^{3}\right)$ with the new vertex $u^{4}=u^{1}+q(2) / 4=\left(\frac{1}{4}, \frac{1}{4}, \frac{1}{2}\right)^{\top}$, $\mathrm{R}=(0,0,0)^{\mathrm{T}}$.

8. Step 1: $\ell\left(u^{4}\right)=2 \in T, \ell\left(u^{4}\right)=\ell\left(u^{3}\right), u^{3}=y^{3}$, i.e. $s=3$.

9. Step 2: $s=3=t+1$ and $R_{\pi_{t}}=R_{1}=0$.

10. step 4: The vertex $y^{3}=u^{3}$ is deleted, $\ell\left(u^{1}\right)=\pi_{2}=1$, i.e. $s^{\prime}=1 ; T=\{2\}$, $\pi(T)=2, \sigma\left(y^{1}, \pi(T)\right)=\sigma^{4}=\sigma\left(u^{1}, u^{4}\right), t=1, \quad s=s^{\prime}=1$.

11. Step 2: the vertex $u^{1}$ is replaced, $\pi(T)=2, \sigma\left(y^{1}, \pi(T)\right)=\sigma^{5}=\sigma\left(u^{4}, u^{5}\right)$ with the new vertex $u^{5}=u^{4}+q(2) / 4=\left(\frac{1}{4}, 0, \frac{3}{4}\right)^{\top}, R=(0,1,0)^{\top}$.

12. Step 1: $\ell\left(u^{5}\right)=1 \notin T$.

13. Step 3: $T=\{1,2\}, \pi(T)=(2,1), \sigma\left(y^{1}, \pi(T)\right)=\sigma^{6}=\sigma\left(u^{4}, u^{5}, u^{6}\right)$ with the new vertex $u^{6}=u^{5}+q(1) / 4=\left(0, \frac{1}{4}, \frac{3}{4}\right)^{\top}, t=2$.

14. Step 1: $\quad \ell\left(u^{6}\right)=3 \notin T$.

15. Step 3: $t=2=n$, a completely labelled simplex is found and the algorithm terminates.

The steps 1-14 are summarized in table 5.2.2.

\subsection{GEOMETRIC INTERPRETATION.}

In this section a geometric interpretation of the variable dimension restart algorithm is given. Observe again that throughout the algorithm $R_{j}=0$ if $j \notin T$ and $R_{j} \geq 0$ if $j \in T$. Therefore it is natural to define the regions $A(T)$ for $T \subset I_{n+1},|T| \leq n$, by

$$
A(T)=\left\{x \in S^{n} \mid x=v+\sum_{j \in T} \lambda_{j} q(j) \text { for nonnegative } \lambda_{j}, j \in T\right\} .
$$




\begin{tabular}{|c|c|c|c|c|c|c|c|c|c|}
\hline Number & $t$ & $T$ & $\pi(T)$ & $\mathrm{R}^{\top}$ & $\sigma$ & $\overline{\mathrm{y}}^{\top}$ & $\ell(\bar{y})$ & $\mathrm{s}$ & $s^{\prime}$ \\
\hline 1,2 & 0 & $\varnothing$ & & $(0,0,0)$ & $\sigma\left(u^{1}\right)$ & $\left(\frac{1}{4}, \frac{3}{2}, \frac{1}{4}\right)$ & 1 & & \\
\hline 3,4 & 1 & $\{1\}$ & (1) & $(0,0,0)$ & $\sigma\left(u^{1}, u^{2}\right)$ & $\left(0, \frac{3}{4}, \frac{1}{4}\right)$ & 2 & & \\
\hline 5,6 & 2 & $\{1,2\}$ & $(1,2)$ & $(0,0,0)$ & $\sigma\left(u^{1}, u^{2}, u^{3}\right)$ & $\left(0, \frac{1}{2}, \frac{1}{2}\right)$ & 2 & 2 & \\
\hline $7,8,9$ & 2 & $\{1,2\}$ & $(2,1)$ & $(0,0,0)$ & $\sigma\left(u^{1}, u^{4}, u^{3}\right)$ & $\left(\frac{1}{4}, \frac{1}{4}, \frac{1}{2}\right)$ & 2 & 3 & \\
\hline 10 & 1 & $\{2\}$ & $(2)$ & $(0,0,0)$ & $\sigma\left(u^{1}, u^{4}\right)$ & & & 1 & 1 \\
\hline 11,12 & 1 & $\{2\}$ & (2) & $(0,1,0)$ & $\sigma\left(u^{4}, u^{5}\right)$ & $\left(\frac{3 \pi}{\pi}, 0, \frac{3}{4}\right)$ & 1 & & \\
\hline 13,14 & 2 & $\{1,2\}$ & $(2,1)$ & $(0,1,0)$ & $\sigma\left(u^{4}, u^{5}, u^{6}\right)$ & $\left(0, \frac{1}{4}, \frac{3}{4}\right)$ & 3 & & \\
\hline
\end{tabular}

Table 5.2.2. The steps of the variable dimension restart algorithm for the example of figure 5.2.1. 
Note that $A(\varnothing)=\{v\}$ and that any point $x$ of $S^{n}$ is a proper point of just one region $A(T)$ where $x \in A(T)$ is called proper if $x \in \AA(T)$ with

$$
\stackrel{\circ}{\AA}(T)=\left\{x \in A(T) \mid x=v+\sum_{j \in T} \lambda_{j} q(j) \text { for positive } \lambda_{j^{\prime}} j \in T\right\} \text {. }
$$

So the regions $\AA(T)$ partition $s^{\mathrm{n}}$.

As described in section 2 , if a set $T$, a permutation $\pi(T)$ and a vector $R$ are generated, the current simplex $\sigma\left(y^{1}, \ldots, y^{t+1}\right)$ is given by $y^{1}=v+\sum_{j=1}^{n+1} R_{j} q(j) / m$ and $y^{i+1}=y^{1}+\sum_{j=1}^{i} q\left(\pi_{j}\right) / m, i=1, \ldots, t$. Since $R_{j}=0$ if $j \notin T$ and $R_{j} \geq 0$ if $j \in T$, the vertices $y^{i}, i=1, \ldots, t+1$, are elements of $A(T)$, whereas $y^{t+1}$ must be a proper point of $A(T)$. Moreover, if $y^{i}$ is a proper point of $A(T)$ then $y^{j}$ is proper for all $j=i+1, \ldots, t+1$. More precisely, let $T(i)$ be the subset of $T$, such that $y^{i} \in \AA(T(i)), i=1, \ldots, t+1$. Then $T(1) \subseteq T(2) \subseteq \ldots \subseteq T(t+1)=T$ with $|T(i+1) \backslash T(i)|$ equal to zero or one. The algorithm generates t-simplices in $A(T)$ if $T$ is the current set of labels. So we have the following corollaries.

COROLLARY 5.3.1. Let $\sigma$ and $\tau$ be two adjacent t-simplices in $A(T)$ generated by the variable dimension restart algorithm. Then the common facet of $\sigma$ and $\tau$ is $T$-complete.

COROLLARY 5.3.2. Let $T$ be the current set of labels, $|T| \leq n$. Then the variable dimension restart algorithm generates t-simplices in a $t$-dimensional linear manifold, being the affine hull of $A(T)$.

The latter corollary emphasizes that the algorithm operates actually with full-dimensional simplices in a t-dimensional linear manifold. The dimension is increased if a new label is found and decreased if a point outside $A(T)$ should be generated and $T$ is the current set of labels.

COROLLARY 5.3.3. Let $T$ be the current set of labels, $|T|<n, \sigma\left(y^{1}, \pi(T)\right)$ a $t$-simplex in $A(T)$ generated by the algorithm and $j \notin T$ a label just found. Then $\sigma\left(y^{1}, \pi(T)\right)$ is a $(T \cup\{j\})$-complete simplex in $A(T)$ and the dimension is increased.

COROLLARY 5.3.4. Let $T$ be the current set of labels, $|T| \leq n$, and $\sigma\left(y^{1}, \pi(T)\right)$ a t-simplex in $A(T)$ generated by the algorithm. If $y^{t+1}$ is the only vertex of $\sigma$ in $\AA(T)$ and $y^{t+1}$ has to be removed then the facet $\tau\left(y^{1}, \ldots, y^{t}\right)$ is a T-complete $(t-1)$ simplex in $A(T \backslash\{k\})$ for some $k \in T$ and the dimension is decreased. 


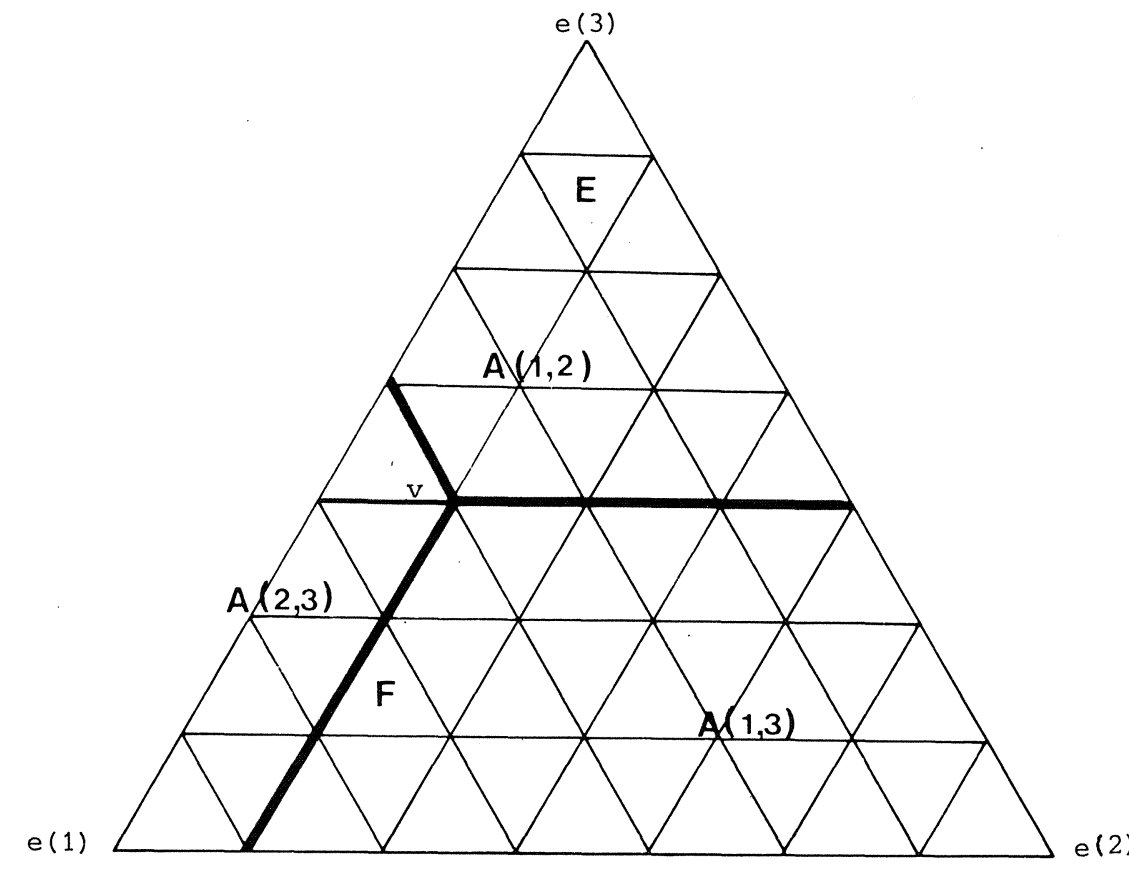

Figure 5.3.1. Geometric interpretation of the variable dimension algorithm.

Ve illustrate the above mentioned features and corollaries by some examples (see figure 5.3.1). $A(T)$ is denoted by $A\left(i_{1}, \ldots, i_{t}\right)$ if $T=$ $\left\{i_{1}, \ldots, i_{t}\right\}$. If the simplex $E$ is generated we have that $R_{1}=R_{2}=2$ and $R_{3}=0$, i.e. $y^{1}=v+\left\{2 q_{I}(1)+2 q(2)\right\} / 7$ is a proper point of $A(1,2)$. Consequently $y^{2}=y^{1}+q(2) / 7$ and $y^{3}=y^{2}+q(1) / 7$ are also proper in $A(1,2)$. Moreover either two vertices have the same label, belonging to $T=\{1,2\}$, whereas the label of the third vertex is equal to the other element of $T$, or $E$ is completely labelled. In the first case $E$ has two adjacent simplices such that the common facets are T-complete.

The vertices of the simplex $F$ are $y^{1}=v+q(3) / 7, y^{2}=y^{1}+q(3) / 7$ and $y^{3}=$ $=y^{2}+q(1) / 7$. If the 1-simplex $\sigma\left(y^{1}, y^{2}\right)$ in $A(3)$ is generated and $\sigma$ is $\{1,3\}-$ complete then the dimension is increased and the simplex $F$ of $A(1,3)$ is generated. Conversely if the 2-simplex $F$ in $A(1,3)$ is generated and $Y^{3}$ has to be removed the dimension is decreased since $\mathrm{y}^{3}$ is the only vertex of $F$ in $\AA(1,3)$ or equivalently in step 2 we have that $s=3$ and $\mathrm{R}_{1}=\mathrm{R}_{\mathrm{t}}=0$. Then the algorithm continues with the one-simplex $\sigma\left(\mathrm{y}^{1}, \mathrm{y}^{2}\right)$ of $A(3)$ by replacing the vertex having label 1 . Observe that replacing the vertex $\mathrm{y}^{3}$ of $\mathrm{F}$ according to table 5.2.1 implies that a simplex in 
$A((T \cup\{2\}) \backslash\{1\})=A(2,3)$ should be generated with $T=\{1,3\}$ the current set of labels. To avoid this the dimension is decreased. In general the dimension is decreased if a replacement step according to table 5.2.1 results in generating a simplex not in $A(T)$ but in an "adjacent" region $A((T \cup\{j\}) \backslash\{k\})$ for some $j \notin T$ and $k \in T$. Then the algorithm continues with simplices in $A(T \backslash\{k\})$.

We conclude this section with some remarks about the path of simplices generated by the algorithm. Let $\mathrm{C}(i)$ be defined by

$$
C(i)=\left\{x \in S^{n} \mid \ell(x)=i\right\} \quad i=1, \ldots, n+1
$$

Let $i \in I_{n+1}$ be the index such that the starting point $v \in C(i)$. Then $\ell(v)=i$ and the algorithm generates one-simplices in $A(i)$ until a grid point $y$ is generated such that $y \in C(j)$ for some $j \neq i$. Then $\ell(y)=j$ and the algorithm continues with 2 -simplices in $A(i, j)$. Suppose that $\tau\left(w^{1}, w^{2}\right)$ is a common facet of two adjacent simplices. Then, for some permutation $\left(h_{i}, h_{j}\right)$ of the set $\{1,2\}, w{ }^{h} \in C(i)$ and $w{ }^{h} \in C(j)$, i.e. $\ell\left(w{ }^{h}\right)=i$ and $\ell\left(w{ }^{h}\right)=j$. So by definition 4.2 .1 we have that

$$
f_{i}\left(w^{h_{i}}\right)-w_{i}{ }_{i} \leq f_{k}\left(w^{h_{i}}\right)-w_{k}{ }_{i} \quad k \neq i
$$

and

$$
f_{j}\left(w^{h} j\right)-w_{j} j \leq f_{k}\left(w^{h} j\right)-w_{k}{ }^{h} \quad k \neq j .
$$

So, roughly speaking, the simplex $\tau\left(w^{1}, w^{2}\right)$ yields an approximation of a point $\mathrm{x}$ such that

$$
f_{i}(x)-x_{i}=f_{j}(x)-x_{j} \leq f_{k}(x)-x_{k} \quad k \neq i, j .
$$

A sequence of approximations of such points is generated until either a point in $C(h), h \neq i, j, i s$ generated $i . e$. a new label is found, or a point outside $A(i, j)$ should be generated by replacing a vertex according to table 5.2.1. In general, if $T$ is the current set of labels and $\tau\left(w^{1}, \ldots, w^{t}\right)$ is a common facet of two adjacent t-simplices generated by the algorithm, for some permutation $\left(h_{j}, j \in T\right)$ of the elements of the set $\{1, \ldots, t\}$ we have 
that $w^{h} j_{\in C}(j), j \in T$. Hence $\tau\left(w^{1}, \ldots, w^{t}\right)$ yields an approximation of a point $x$ such that

$$
f_{i}(x)-x_{i}=f_{j}(x)-x_{j} \quad \text { for all } i, j \in T
$$

and

$$
f_{i}(x)-x_{i} \leq f_{k}(x)-x_{k} \quad \text { for all } i \in T, k \notin T \text {. }
$$

Note that $x$ is such a point if $x \in C(T)$, where $C(T)=n_{j \in T} \bar{C}(j)$ with $\bar{C}(j)$ the closure of $C(j)$. Observe that it is possible that $C(T) \cap \tau=\varnothing$. Againa sequence of approximations of such points is generated until either a point in $C(h), h \notin T$, is generated or a simplex having a $T$-complete facet in $A(T\{k\})$, for some $k \in T$, is generated. In the first case the algorithm continues with simplices in $A(T U\{h\})$ and generates a sequence of approximations of points $x \in C(T \cup\{h\})$, whereas in the latter case the algorithm proceeds with simplices in $A(T\{k\})$, generating a sequence of approximations of points $x \in C(T\{k\})$. In figure 5.3.2, $\bar{C}(i), i=1,2,3$, and the regions $A(T), T \subset I_{n+1}$, are given. The set $S^{n}$ is triangulated with grid size $1 / 9$. Only the grid points and the completely labelled simplex generated by the algorithm are given.

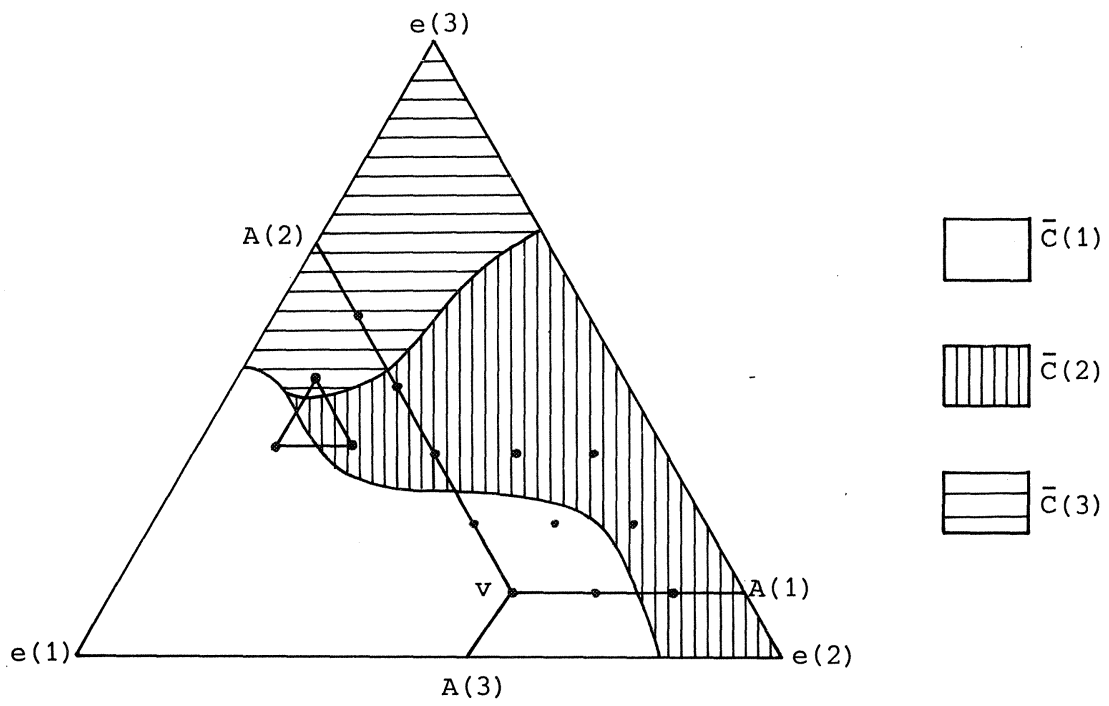

Figure 5.3.2. $n=2, v=(1 / 3,5 / 9,1 / 9)^{\top}, m=9$. The dots are the grid points generated by the algorithm. 


\subsection{CONVERGENCE OF THE ALGORITHM.}

In this section it is proved that the variable dimension restart algorithm always succeeds in finding a completely labelled simplex by establishing that all steps are feasible and the algorithm never returns in a simplex visited already before.

First, it is shown that a replacement step according to table 5.2.1 is always feasible. Clearly, if a vertex of the t-dimensional simplex $\sigma\left(y^{1}, \ldots, y^{t+1}\right)$ has to be replaced according to table 5.2.1, the replacement step produces again a t-simplex of the triangulation of $s^{n}$ except when all other vertices are points on some boundary $s_{j}^{n}$. suppose for some $s, s \leq t+1, y^{s}$ is the vertex to be replaced and assume that, for some $j$, $y^{S}$ is the only vertex not on the $j$-th boundary. Then $y_{j}^{i}=0$ for all $i \neq s$. If the starting point is not on the $j$-th boundary, $j$ must be an element of $T$, otherwise the $j$-th component could not have been decreased. Since the common facet of two adjacent $t$-simplices is $T$-complete, one of the vertices $y^{i}$ must have label $j$. Because of the proper labelling $\ell\left(y^{i}\right) \neq j, i \neq s$. Hence $y^{s}$ is the only vertex with label $j$ and cannot be replaced. This proves that the replacement step is feasible if the starting point is not on the $j$-th boundary.

If $v$ is an element of $s_{j}^{n}$, either $j$ is an element of $T$ or it is not. The first case is identical to the case just mentioned. In the other case, $s$ must be equal to $t+1$, since otherwise $0=y_{j}^{s+1}=y_{j}^{s}-m^{-1}$ which is only possible if $j \in T$. Moreover we have that $R_{\pi_{t}}=0$ since $\pi_{t}=j-1$ and $y_{j}^{1}=v_{j}+R_{j-1} / m$ $=0$. So step 4 should be performed contradicting the fact that $y^{t+1}$ must be replaced according to table 5.2.1. These facts together prove that every replacement step is feasible. Moreover, a vertex obtained in step 3 by extending a $t$-dimensional simplex to a $(t+1)$-dimensional simplex $(t \leq n-1)$ is a point in $s^{n}$. To prove this, let $\sigma\left(y^{1}, \ldots, y^{t+1}\right)$ be a $t$-dimensional ( $T \cup\{j\}$ )-complete simplex in $A(T)$ generated by the algorithm, where $j \notin T$ is the new label. So $\ell\left(y^{s}\right)=j$ for some $1 \leq s \leq t+1$. Because of the proper labelling rule $y_{j}^{S}>0$. Since $j$ is not an element of $T, \pi_{h} \neq j$ for $h=1, \ldots, t$ implying that $y_{j}^{t+1}$ is also positive. Hence the $j$-th component of the new vertex $y^{t+2}=$ $y^{t+1}+q(j) / m$ is nonnegative and therefore $y^{t+2}$ must be a point in $s^{n}$. Consequently, all steps in the algorithm are feasible.

Next we prove that the algorithm never generates a simplex visited already before. Let $\sigma^{0}, \sigma^{1}, \sigma^{2}, \ldots$ be the sequence of simplices generated by the algorithm, so $\sigma^{0}=\{v\}$. We prove that each generated simplex $\sigma^{i}, i \geq 1$, can 
be entered from just two adjacent simplices, while $\sigma^{0}$ can be entered from just one adjacent simplex. Then by the standard arguments of section 4.2 the algorithm can never revisite a simplex. For given $i \geq 1$, let $\sigma^{i}$ be a t-dimensional simplex in a region $A(T)$. Now two cases can occur.

a) $\sigma^{i}$ has just two (t-1)-dimensional $T$-complete facets b) $\sigma^{i}$ is $(T \cup\{k\})$-complete for some $k k T$, and $\sigma^{i}$ has exactly one (t-1)-dimensional $T$-complete facet.

In the first case $\sigma^{i}$ has exactly two different adjacent simplices from which it can be entered by the algorithm. Both simplices are simplices in $A(T)$ or one is a simplex in $A(T \backslash\{j\})$ for some $j \in T$ and the other is a simplex in $A(T)$. In the second case $\sigma^{i}$ has also exactly two adjacent simplices from which it can be entered, one $(t+1)$-dimensional simplex in $A(T \cup\{k\})$ whereas the other one is either a simplex in $A(T)$ or in $A(T \backslash\{j\})$ for some $j \in T$. So in all cases, $\sigma^{i}$ is entered from thesimplex $\sigma^{i-1}$ and there is just one other simplex $\sigma^{i+1}$ which can be entered from $\sigma^{i}$. Moreover $\sigma^{0}$ is a zero-dimensional simplex which is $(T \cup\{k\})$-complete with $T=\varnothing$ and $k=\ell(v)$. Since $T=\not,, \sigma^{0}$ has no $T$-complete facet. Therefore $\sigma^{0}$ can be entered from just one other simplex being the simplex $\sigma^{1}=\sigma\left(\mathrm{y}^{1}, \mathrm{y}^{2}\right)$ with $\mathrm{y}^{1}=\mathrm{v}$ and $\mathrm{y}^{2}=\mathrm{v}+\mathrm{q}(\ell(\mathrm{v})) / \mathrm{m}$.

Since the number of $t$-simplices, $0 \leq t \leq n$, is finite the algorithm must terminate within a finite number of steps with a completely labelled n-simplex.

\subsection{INTERPRETATION OF THE ALGORITHM IN $S^{\mathrm{n}} \times[0,1]$.}

The variable dimension restart algorithm can be easily put in the framework of other restart algorithms by adding an extra level of points. This interpretation was independently found by Barany [1979a], Van der Laan and Talman [1979a] and Todd [1978b]. In Van der Laan and Talman [1978a] the variable dimension restart algorithm is applied to compute a fixed point of a function in the product space of unit simplices. Interpretations with an extra level of points of this more general case can be found in van der Laan and Talman [1979 $\mathrm{C}$. An interpretation of a closely related algorithm on $\mathrm{R}^{\mathrm{n}}$ (c.f. Van der Laan and Talman [1978b]) was given by Todd and "iright [1979] and Barany [1979b] (see also Talman [1980]). 
Using an extra level of points we triangulate $s^{n} \times[0,1 . \bar{j}$, where the extra level $s^{\mathrm{n}} \times\{0\}$ is not triangulated (or triangulated with grid size one) and the natural level $\mathrm{S}^{\mathrm{n}} \times\{1\}$ is triangulated with grid size $\mathrm{m}^{-1}$. So, the only grid points on the zero level are the vertices ê $(i)=\left(e(i)^{\top}, 0\right)^{\top}, i=1, \ldots, n+1$. Now we give the triangulation.

THEOREM 5.5.1. Let $\mathrm{G}$ be the collection of $(n+1)-$ simplices obtained by

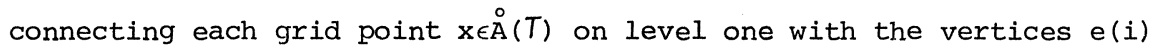
$i \notin T$ on level zero. Then, $G$ is a triangulation of $S^{n} \times[0,1]$.

PROOF. In this proof we use the following notation. A point of $s^{n} \times[0,1]$ is denoted by $(x, t)$ where $x \in S^{n}$ and $t \epsilon[0,1]$. The set $E(T)$ denotes the set of vertices $\hat{e}(i), i \notin T$.

We prove now that each point $(x, t)$ lies in a simplex of $G$ and that the intersection of two simplices of $G$ is a face of each. Since the collection of regions $\AA(T)$ partition $S^{n}$, there is for given $(x, t)$ a unique subset $T$ of $I_{n+1}$, such that $x \in \AA(T)$. Moreover, for any $j \in T$ there are unique numbers $\alpha_{j}$ and $\lambda_{j}(k), k \in T \backslash\{j\}$, and nonnegative numbers $\beta_{j}(i), i \notin T$, such that

$$
x=\alpha_{j} v+\sum_{k \in T \backslash\{j\}} \lambda_{j}(k) q(k)+\sum_{i \notin T} \beta_{j}(i) e(i)
$$

Since $x \in \stackrel{\circ}{(T)}$ we have that $\alpha_{j} \leq 1$. Let $\bar{T}$ be a subset of $T$ such that

$$
\begin{aligned}
& \text { (i) } j \in \bar{T} \text { if } \alpha_{j}<t \\
& \text { (ii) } j \notin \bar{T} \text { if } \alpha_{j}>t .
\end{aligned}
$$

Clearly, the intersection of the affine hull of the elements of $E(\bar{T})$ and $(x, t)$ with the region $\AA(\bar{T})$ on the one level is a point, say $(\bar{x}, 1)$. Let $\sigma\left(y^{1}, \pi(\bar{T})\right)$ be a $\bar{t}$-simplex containing $\bar{x}$. Then $(x, t)$ lies in the $(n+1)-$ simplex $\sum\left(\sigma\left(y^{1}, \pi(\bar{T})\right)\right)$ defined as the simplex with vertices the elements of $E(\bar{T})$ and the vertices of $\sigma$ on the one level. This proves the first part of the lemma.

Next, suppose $\Sigma_{1}$ and $\Sigma_{2}$ are two simplices of $G$ both containing $(x, t)$. Let $\sigma_{1}$ and $\sigma_{2}$ be the corresponding simplices on the one level, i.e. $\sigma_{i} \times\{1\}=\Sigma_{i} \cap\left(S^{n} \times\{1\}\right), i=1,2$. Then $(x, t)$ is contained in the face with vertices the elements of $E\left(T^{*}\right)$ and the vertices of $\sigma_{1} \cap \sigma_{2}$ on the one-level, 
where $T^{*}$ is the largest subset of $T$ such that (i) and (ii) are satisfied. clearly, this face is a face of both $\Sigma_{1}$ and $\Sigma_{2}$.

The triangulation of $s^{n} \times[0,1]$ is illustrated for $n=1, m=8, v=(3 / 8,5 / 8)^{\top}$ in figure 5.5.1 and for $n=2, m=3$ and $v=(1 / 3,1 / 3,1 / 3)^{\top}$ in figure 5.5.2.

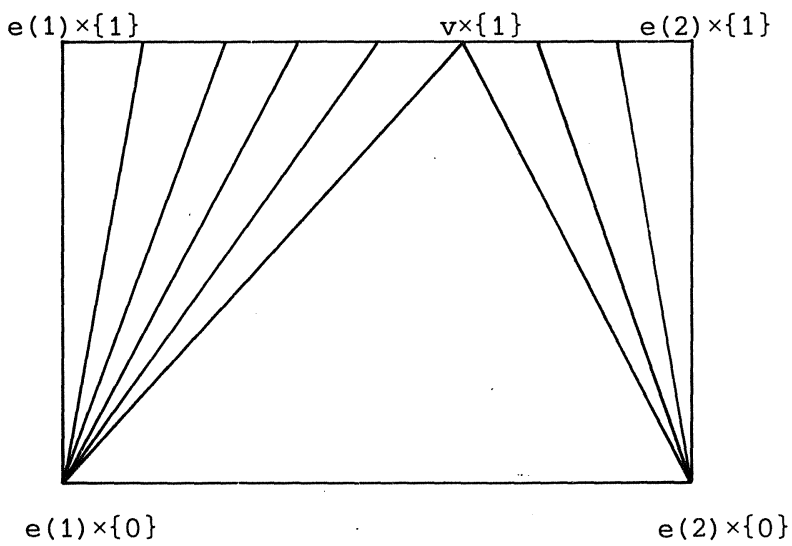

Figure 5.5.1. Triangulation of $s^{1} \times[0,1], m=8, v=(3 / 8,5 / 8)^{\top}$.

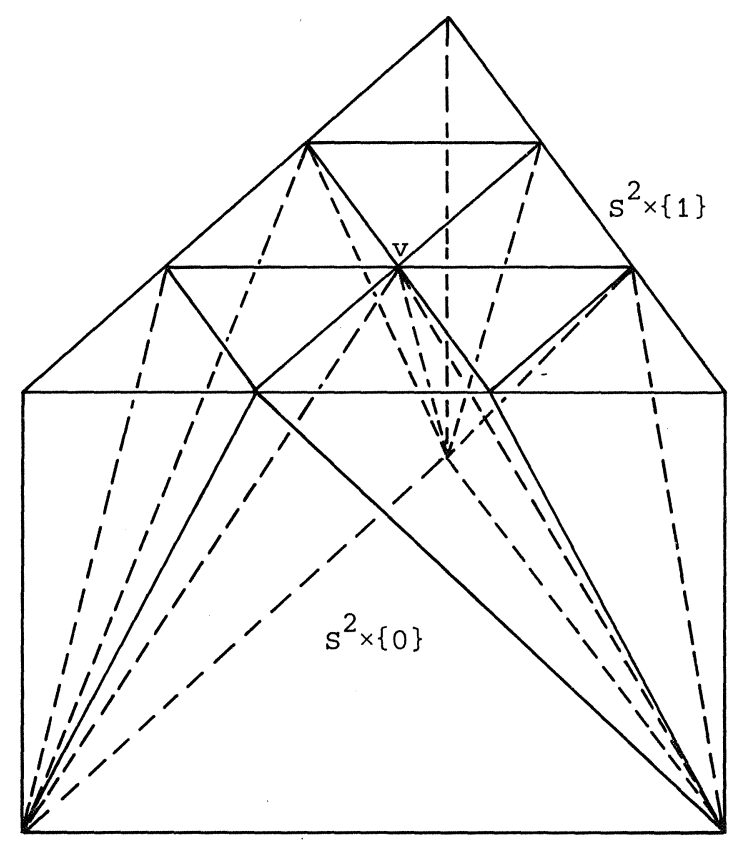

Figure 5.5.2. Triangulation of $s^{2} \times[0,1], m=3, v=(1 / 3,1 / 3,1 / 3)^{\top}$. 
The grid points of $s^{n} \times[0,1]$ are labelled according to the standard labelling on $s^{n}$, i.e. $\ell(\hat{e}(i))=i, i=1, \ldots, n+1$ and $\ell((x, 1))=\ell(x)$ for a point $(x, 1)$ in $s^{n} \times\{1\}$. Clearly, $s^{n} \times\{0\}$ is a completely labelled $n$-simplex and is a facet of just one $(n+1)$-simplex of the triangulation of $s^{n} \times[0,1]$. This simplex is the convex hull of $v \times\{1\}$ and $s^{n} \times\{0\}$. Starting with this simplex a path of adjacent $(n+1)$-simplices is generated by replacing in every generated simplex the vertex having the same label as the new vertex. Using standard arguments the algorithm must terminate with a simplex having a completely labelled facet on the boundary of $s^{\mathrm{n}} \times[0,1]$ and this facet cannot be the facet $s^{n} \times\{0\}$. Because of the proper labelling there are no completely labelled $n$-simplices in $\left(b d s^{n}\right) \times[0,1]$, i.e. there is no completely labelled $n$-simplex $\sigma$ such that, for some $i, x_{i}=0$ for all $x \in \sigma$. Hence, the algorithm terminates with an ( $n+1)$-simplex having a completely labelled facet in $s^{\mathrm{n}} \times\{1\}$. This facet yields a good approximation of a fixed point. The intersection of the path of $(n+1)$-simplices with $s^{n} \times\{1\}$ gives the sequence of simplices of the variable dimension restart algorithm. Observe that when in the variable dimension restart method a new label $\mathrm{k}$ is found, the current simplex $\sigma\left(\mathrm{y}^{1}, \ldots, \mathrm{y}^{\mathrm{t}+1}\right)$ is extended to the simplex $\sigma\left(y^{1}, \ldots, y^{t+2}\right)$ with $y^{t+2}=y^{t+1}+q(k) / m$. In the interpretation with an artificial level the vertex of $s^{\mathrm{n}} \times\{0\}$ having label $k$, that is the vertex $\hat{\mathrm{e}}(\mathrm{k})$, is replaced by $\mathrm{y}^{\mathrm{t}+2}$. Also, if in the variable dimension restart algorithm the vertex $y^{t+1}$ of a simplex $\sigma\left(y^{1}, \ldots, y^{t+1}\right)$ should be removed while $R_{\pi_{t}}=0$, the vertex $y^{t+1}$ is deleted and the vertex $y^{i}, i \leq t$, having label $\pi_{t}$ is removed. In the method with the artificial level $y^{t+1}$ on the one-level is replaced by $\hat{e}\left(\pi_{t}\right)$ and in the next step the vertex $y^{i}$ having label $\pi_{t}$ is replaced since $\ell\left(\hat{e}\left(\pi_{t}\right)\right)=\pi_{t}$.

Although the variable dimension restart algorithm method can be interpreted as a method with an extra level as just described, this level does not have an artificial influence on the path and is not needed to prove the convergence. Moreover there are only $n+1$ extra points, whereas in the Sandwich method the number of extra points, which are artificially labelled, is of the order of the number of grid points on the natural level. This means that even if the variable dimension restart method is applied with an extra level, the number of grid points on this level, generated by the algorithm, is very small (in most examples zero). Observe that decreasing the dimension corresponds to generating a grid point on the extra level. In the Sandwich method the number of grid points on the extra 
level generated by the algorithm is of the same order as the number of grid points that is generated on the natural level. In particular this is a disadvantage if vector labelling is used since then each grid point generated by the algorithmimplies that a linear programming step should be made.

\subsection{VECTOR LABELLING.}

In this section we discuss the application of the variable dimension restart algorithm with vector labelling. Each point $\mathrm{x}$ of $\mathrm{s}^{\mathrm{n}}$ receives the standard vector label of definition 4.11 .1 with $b=e$, i.e.

$$
\ell(x)=-f(x)+x+e
$$

where $f(x)$ is a piecewise linear approximation to the mapping $\phi$ with respect to the standard triangulation of $s^{n}$ with grid size $m^{-1}$. Let $T$ be a subset of $I_{n+1}$ with $|T|=t$. Then we have the following definition.

DEFINITION 5.6.1. A $(t-1)$-simplex $\sigma$ with vertices $w^{1}, \ldots, w^{t}$ is called $T$-complete if the system of linear equations

$$
\sum_{i=1}^{t} \lambda_{i} l\left(w^{i}\right)+\sum_{h \notin T} \mu_{h} e(h)=e
$$

has a nonnegative solution $\lambda_{i}^{*}, i=1, \ldots, t$ and $\mu_{h}^{*}, h \notin T$.

Observe that any zero-dimensional simplex $\sigma(w)$ is $i_{0}$-complete with $i_{0}$ the index such that $l_{i_{0}}(w)=\max _{j} l_{j}(w)$. The solution of the system is $\lambda_{1}^{*}=\left\{l_{i_{0}}(w)\right\}^{-1}$ and $\mu_{h}^{*}=1-\left\{l_{h}(w) / l_{i_{0}}(w)\right\}, \quad h \neq i_{0}$. The index $i_{0}$ is unique if $l_{i_{0}}(w)>\ell_{j}(x), j \neq i_{0}$. If $i_{0}$ is not unique there are more solutions and the system of linear equations is degenerated. In the following we assume that degeneracy does not occur (see also Todd [1976a]).

LEMMA 5.6.2. For any $T$-complete simplex $\sigma\left(w^{1}, \ldots, w^{t}\right)$ holds

$$
\text { (n+1) } \sum_{i=1}^{t} \lambda_{i}^{*}+\sum_{h \notin T} \mu_{h}^{*}=n+1
$$

with $\lambda_{i}^{*}, i=1, \ldots, t ; \mu_{h}^{*}, h \notin T$, the solution of the system of linear equations. 
PROOF. From definition 5.6.1 and the labelling rule we have that

$$
\sum_{i=1}^{t} \lambda_{i}^{*}\left(-f\left(w^{i}\right)+w^{i}+e\right)+\sum_{h \notin T} \mu_{h}^{*} e(h)=e .
$$

Summing up over all components we obtain

$$
\sum_{i=1}^{t} \lambda_{i}^{*}\left[\sum_{j=1}^{n+1}\left\{-f_{j}\left(w^{i}\right)+w_{j}^{i}+1\right\}+\sum_{h \notin T} \mu_{h}^{*}=n+1 .\right.
$$

Since, for all $i, \sum_{j=1}^{n+1} f_{j}\left(w^{i}\right)=\sum_{j=1}^{n+1} w_{j}^{i}=1$, the lemma now follows immediately.

It is obvious that if $T=I_{n+1}$, a $T$-complete simplex $\sigma\left(w^{1}, \ldots, w^{n+1}\right)$ is completely labelled. Then $\sum_{i=1}^{n+1} \lambda_{i}^{*}=1$ en $\sum_{i=1}^{n+1} \lambda_{i}^{*} w^{i}$ is an approximation of a fixed point of the mapping $\phi$ (see lemma 4.11.2).

We are now ready to describe the algorithm for vector labelling. Again the algorithm starts with an arbitrarily chosen grid point $\mathrm{v}$ of the triangulation of $S^{n}$. Moreover the system of linear equations is set equal to $I \mu=e$ with $I$ the $n+1$ identity matrix. By alternating linear programming and replacement steps a path of adjacent simplices of variable dimension is generated until a completely labelled simplex is found. Formally, the algorithm is described by the following steps.

Step 0. Set $t=0, y^{1}=v, T=\varnothing, \sigma=\sigma^{0}\left(y^{1}, \pi(\phi)\right)$ and $R_{j}=0, j=1, \ldots, n+1$. Set $\mathrm{p}=1$ and $\mu_{h}=1, h \in I_{n+1}$.

Step 1. Calculate $\ell\left(y^{p}\right)$. Make a linear programming step by bringing $\ell\left(y^{p}\right)$ in the system of linear equations.

$$
\begin{aligned}
& \sum_{\substack{i=1 \\
i \neq p}}^{t+1} \lambda_{i} \ell\left(y^{i}\right)+\sum_{h \notin T} \mu_{h} e(h)=e . \\
& \text { If } e(j) \text { is eliminated for some } j \notin T \text { go to step } 3 \text {. Otherwise } \ell\left(y^{s}\right) \\
& \text { is eliminated for just one vertex } y^{s} \neq y^{p} \text {. }
\end{aligned}
$$

Step 2. If $s=t+1$ and $R_{\pi_{t}}=0$ go to step 4. Otherwise $\sigma\left(y^{1}, \pi(T)\right.$ ) and $R$ are adapted according to table 5.2 .1 by replacing $\mathrm{y}$ \% Return to step 1 with $p$ the index of the new vertex of $\sigma$. 
Step 3. If $t=n$, a completely labelled simplex is found and the algorithm terminates. If $t<n, a(T \cup\{j\})$-complete simplex is found and the dimension is increased. $T$ becomes $T u\{j\}, \pi(T)$ becomes $\left(\pi_{1}, \ldots, \pi_{t}, j\right), \sigma$ becomes $\sigma\left(y^{1}, \pi(T)\right)$. Then $t$ becomes $t+1$ and return to step 1 with $\mathrm{p}=\mathrm{t}+1$.

step 4. The dimension is decreased. A linear programming step is made by bringing $e\left(\pi_{t}\right)$ in the set of linear equations

$$
\sum_{i=1}^{t} \lambda_{i} l\left(y^{i}\right)+\sum_{h \notin T} \mu_{h} e(h)=e .
$$

Either for some $j \notin T, e(j)$ is eliminated or for some $s^{\prime} \leq t, \ell\left(y^{s^{\prime}}\right)$ is eliminated. Now $T$ becomes $\left(T \backslash\left\{\pi_{t}\right\}\right), \pi(T)$ becomes $\left(\pi_{1}, \ldots, \pi_{t-1}\right)$ and $\sigma$ becomes $\sigma\left(y^{1}, \pi(T)\right)$. Then $t$ becomes $t-1$. Return to step 3 if for some $j, e(j)$ has been eliminated. Otherwise return to step 2 with $s=s^{\prime}$.

Analogously to the integer labelling case, a search is made in the directions $i$ if the vectors $e(i)$ are eliminated. More precisely if for a certain subset $T$ of $I_{n+1}$ a unit vector $e(j), j \notin T$, is eliminated in step 1 , we have that for the permutation $\pi(T)$ and the simplex $\sigma\left(\mathrm{y}^{1}, \pi(T)\right.$ ) generated by the algorithm the conditions a-d of section 2 are satisfied. Then, if $t<n, T$ is set equal to $(T \cup\{j\})$ and a search is made in the directions $i$, $i \in T$, by generating a path of simplices in $A(T)$ with $T$-complete common facets until either again a unit vector is eliminated or a simplex having a $T$-complete facet in $A(T \backslash\{k\})$ is generated for some $k \in T$. In the latter case a linear programming step is made with $e(k)$. Note that a unit vector can be eliminated by another one, implying that the algorithm continues with simplices in an "adjacent" equal-dimensional region, which behaviour is not possible in case of integer labelling.

We prove now that the algorithm must find a completely labelled simplex. First we show that the algorithm never returns to a simplex generated already before. Let $\sigma_{i}, i \geq 1$ be a $t$-dimensional simplex of the "sequence $\sigma_{1}$ ' $\sigma_{2}, \ldots$ of generated simplices in the region $A(T), T \neq \emptyset$. Then either

a) $\sigma_{i}$ has just two $(t-1)$-dimensional $T$-complete facets

or

b) $\sigma_{i}$ has just one $(t-1)$-dimensional $T$-complete facet and $\sigma_{i}$ is $(T \cup\{k\})$-complete for some $k$ not in $T$. 
As in the case of integer labelling, in both cases $\sigma_{i}$ can be entered from only two adjacent simplices. Moreover the starting simplex $\sigma(v)$ can be entered from only one adjacent simplex being $\sigma\left(\mathrm{y}^{1}, \mathrm{y}^{2}\right)$ where $\mathrm{y}^{1}=\mathrm{v}$ and $y^{2}=v+q\left(i_{0}\right) / m$ if $e\left(i_{0}\right)$ is eliminated by $l(v)$ in step 1 .Together these observations prove that the algorithm can never return to a previously generated simplex.

Next we prove that all steps are feasible. Clearly, all linear programming steps are feasible. Next we show that a replacement step according to table 5.2.1. is feasible. Let $\sigma\left(y^{1}, \ldots, y^{t+1}\right)$ be a simplex generated by the algorithm and let $y^{s}, 1 \leq s \leq t+1$, be the vertex to be replaced. This step is feasible unless for some $j \in I_{n+1}, y^{1}, \ldots, y^{s-1}, y^{s+1}, \ldots, y^{t+1}$ are elements of $s_{j}^{n}$ and $y_{j}^{S}>0$.

THEOREM 5.6.3. Let $\sigma\left(\mathrm{y}^{1}, \ldots, \mathrm{y}^{\mathrm{t}+1}\right)$ be a $t$-dimensional simplex such that for some $j y_{j}^{i}=0$ for $i \neq s$. If $v_{j} \neq 0$ the system of linear equations

$$
\sum_{\substack{i=1 \\ i \neq s}}^{t+1} \lambda_{i} l\left(y^{i}\right)+\sum_{h \notin T} \mu_{h} e(h)=e
$$

has no feasible solution.

PROOF. If the system of linear equations has a feasible (nondegenerated) solution $\lambda_{i}^{*}$, iłs, and $\mu_{h}^{*}, h \notin T$, we have from the proof of lemma 5.6.2 that

$$
(n+1) \sum_{\substack{i=1 \\ i \neq s}}^{t+1} \lambda_{i}^{*}+\sum_{h \notin T} \mu_{h}^{*}=n+1,
$$

implying that $\sum \lambda_{i}^{*}<1$. Since the starting point $v$ is not on the $j-$ th boundary of $s^{n}, j$ must be an element of $T$, i.e. e(j) is eliminated already. So, the $j$-th equation of the system yields

$$
\sum_{\substack{i=1 \\ i \neq s}}^{t+1} \lambda_{i}^{*} l_{j}\left(y^{i}\right)=\sum_{\substack{i=1 \\ i \neq s}}^{t+1} \lambda_{i}^{*}\left(-f_{j}\left(y^{i}\right)+y_{j}^{i}+1\right)=1
$$

Since $y_{j}^{i}=0$ and $f_{j}\left(y^{i}\right) \geq 0$, this implies that $\sum_{i \neq s} \lambda_{i}^{*} \geq 1$ which gives a contradiction.

The theorem proves that a vertex has not to be replaced if all other vertices are on a same boundary given that the starting point is not 
on this boundary. If, however, $v_{j}=0$ either $j \in T$ or $j \notin T$. The first case is identical to the case just discussed. As shown for integer labelling the latter case implies that $s=t+1$ and $R_{\pi_{t}}=0$, so that $y^{t+1}$ has to be deleted and a linear programming step has to be made with e ( $\left.\pi_{t}\right)$. Hence all replacement steps according to table 5.2.1 are feasible. Finally we prove that the extension to a higher dimensional simplex is always feasible. Let for some $j \notin T, \sigma\left(y^{1}, \ldots, y^{t+1}\right)$ be a $t$-dimensional $(T \cup\{j\})$-complete simplex of $A(T)$. Since $j \notin T, \pi_{h} \neq j, h=1, \ldots, t$. Hence if $y_{j}^{t+1}=0, y_{j}^{i}=0$ for all $i$. Then analogous to the proof of theorem 5.6.3, e(j) could not have been eliminated. Hence, $y_{j}^{t+1}>0$ and the new vertex $y^{t+2}=y^{t+1}+q(j) / m$ is a grid point of $\mathrm{s}^{\mathrm{n}}$. Consequently, all steps are feasible.

By the fact that all steps are feasible and that the algorithm can never return to a previously generated simplex, a completely labelled simplex must be found, since the number of simplices is finite.

The variable dimension algorithm applied with vector labelling generates a path of simplices of variable dimension such that if a simplex of $A(T)$ is generated we have that the unit vectors $e(j), j \in T$, have been eliminated. This means that the common facet of two adjacent simplices in $A(T)$, generated by the algorithm, is $T$-complete. Let $\tau\left(w^{1}, \ldots, w^{t}\right)$ be such a common facet of two adjacent simplices in $A(T)$. Since $\tau$ is $T$-complete we have that the system of linear equations

$$
\sum_{i=1}^{t} \lambda_{i} l\left(w^{i}\right)+\sum_{h \notin T} \mu_{h} e(h)=e
$$

has a nonnegative solution $\lambda_{i^{\prime}}^{*} i=1, \ldots, t, \mu_{h^{\prime}}^{*} h \notin T$. From lemma 5.6 .2 we have that $\Sigma_{i=1}^{t} \lambda_{i}^{*}<1$. Define $\alpha=\left(1-\sum_{i=1}^{t} \lambda_{i}^{*}\right) / \Sigma_{i=1}^{t} \lambda_{i}^{*}$ and define $\bar{\lambda}_{i}=\lambda_{i}^{*} / \Sigma_{i=1}^{t} \lambda_{i}^{*}$. Clearly $\alpha>0$ and $\sum_{i=1}^{t} \bar{\lambda}_{i}=1$.

From (5.6.1) it follows that for $j \in T$ holds

$$
\sum_{i=1}^{t} \lambda_{i}^{*} l_{j}\left(w^{i}\right)=\sum_{i=1}^{t} \lambda_{i}^{*}\left\{-f_{j}\left(w^{i}\right)+w_{j}^{i}+1\right\}=1
$$

or equivalently

$$
\sum_{i=1}^{t} \bar{\lambda}_{i}\left\{-f_{j}\left(w^{i}\right)+w_{j}^{i}\right\}=\alpha
$$

Since $f$ is a piecewise linear approximation to the mapping $\phi$ we have that 
(5.6.2) $\quad f_{j}(\bar{x})-\bar{x}_{j}=-\alpha \quad j \in T$

with $\bar{x}=\sum_{i=1}^{t} \bar{\lambda}_{i} w^{i}$. For $h \notin T$ the $h$-th equation of (5.6.1) can be rewritten as

$$
\sum_{i=1}^{t} \bar{\lambda}_{i}\left\{-f_{h}\left(w^{i}\right)+w_{h}^{i}\right\}=\alpha-\mu_{h}^{*} / \Sigma_{i=1}^{t} \lambda_{i}^{*} .
$$

Since $\mu_{h}>0$ it follows that

$$
f_{h}(\bar{x})-\bar{x}_{h}>-\alpha \quad h \notin T
$$

So by generating a sequence $\sigma_{1}, \sigma_{2}, \ldots$ of simplices in $A(T)$ the variable dimension restart algorithm traces a path of points $x \in A(T)$ such that for some $\alpha(x)(5.6 .2)$ and (5.6.3) hold, until $f_{h}(x)-x_{h}$ becomes equal to $\alpha(x)$ for some $h \notin T$, i.e. $e(h)$ is eliminated, or the path crosses a region $A(T \backslash\{k\})$ for some $k \in T$ in which case a linear programming step is made with $e(k)$. Recall from the end of section 3 that there is an analogous behaviour in case of integer labelling.

The interpretation with an extra level with $n+1$ grid points is straightforward if the vertex $\hat{e}(i)$ of $s^{n} \times\{0\}$ is labelled with the vector $e(i)$ $i=1, \ldots, n+1$. Then the method starts with the $(n+1)$-simplex being the convex hull of $s^{n} \times\{0\}$ and $v \times\{1\}$. The facet $s^{n} \times\{0\}$ of this simplex is completely labelled with $\mu_{j}^{*}=1, j=1, \ldots, n+1$, as the solution of the system of linear equations. By alternating linear programming and replacement steps a sequence of $(n+1)$-simplices is generated such that the common facets are completely labelled. This sequence terminates with an $(n+1)-$ simplex having a completely labelled facet in $\mathrm{s}^{-\mathrm{n}} \times\{1\}$. In Van der Laan and Talman [1979c] and Talman [1980] it is proved that the method can be seen as a method which traces a path of zeroes of a piecewise linear approximation $f(x, t)$ to a mapping $\bar{\phi}(x, t)$ with respect to the triangulation of $s^{n} \times[0,1]$, where $\bar{\phi}(x, t)$ is such that

$$
\bar{\phi}(x, 0)=\{(n+1) x-e\} \text { and } \bar{\phi}(x, 1)=\{x\}-\phi(x) \text {. }
$$

It is not necessary to initiate the variable dimension restart algorithm with the system of linear equations $I \mu=e$. Another possibility is to start the algorithm with the system of linear equations $A \mu=e$ where $a(i)=\ell(e(i))=e(i)-\tilde{f}(e(i))+e$ and $\tilde{f}$ is a linear approximation to the 
mapping $\phi$ with respect to the standard triangulation with grid size 1. Clearly this system has a unique solution since $\tilde{f}$ has a fixed point on $s^{n}$ (see lemma 4.11.2).

\subsection{CONVERGENCE CONDITIONS FOR MAPPINGS ON $\mathrm{T}^{\mathrm{n}}$.}

In the previous sections we discussed the variable dimension restart algorithm to approximate a fixed point of a function or mapping from the compact convex unit simplex into itself. The generalization of the method to compute a fixed point. of a function or mapping from $\mathrm{T}^{\mathrm{n}}$ to itself can easily be done. Of course $\mathrm{T}^{\mathrm{n}}$ can be triangulated by the standard triangulation as defined in definition 3.4.3. However, here we use the T. triangulation given in section 3.4 which seems to be much more appropriate to be used in fixed point algorithms. So $T^{\text {n }}$ is triangulated according to the $T$ triangulation with grid size $\delta$ for some positive number $\delta$ and with respect to some point $z \in T^{n}$. In the following $z$ will be taken as the starting point $v$, which can be arbitrarily chosen. Analogously to section 2 we redefine the matrix $T$ as the $(n+1) \times(n+1)$ matrix

$$
\mathrm{T}=\left[\begin{array}{rrrrrr}
-\mathrm{n} & 1 & \cdot & \cdot & \cdot & 1 \\
1 & -\mathrm{n} & & & & \cdot \\
\cdot & & \cdot & & & \cdot \\
\cdot & & & \cdot & & \cdot \\
1 & \cdot & \cdot & \cdot & 1 & -\mathrm{n}
\end{array}\right] .
$$

The algorithm starts in $\mathrm{v}$ and operates exactly in the same way as the variable dimension restart method on $s^{n}$ does, except that $q(i)$ is changed into $t(i), i=1, \ldots, n+1$. So, a path of adjacent simplices of variable dimension is generated such that the common facet of two adjacent t-simplices $\sigma_{1}$ and $\sigma_{2}$ generated by the algorithm is T-complete if $\sigma_{1}$ and $\sigma_{2}$ are two simplices in $A(T)$. The regions $A(T), T \subset I_{n+1^{\prime}}$ are redefined by

$$
A(T)=\left\{x \in T^{n} \mid x=v+\sum_{j \in T} \lambda_{j} t(j) \text { for nonnegative } \lambda_{j^{\prime}} j \in T\right\} .
$$

Observe that the regions $A(T)$ depend on the underlying triangulation. Again the dimension is increased if a ( $T \cup\{j\})$-complete simplex in $A(T)$ is generated and the dimension is decreased if a simplex in $A(T)$ is generated 
having a $T$-complete facet in $A(T \backslash\{k\})$ for some $k \in T$. Of course all steps are feasible. Moreover a simplex can only be visited once. Hence the sequence of simplices either terminates with a completely labelled simplex or goes to infinity. First we give sufficient conditions to guarantee that the algorithm terminates in case of integer labelling. Define for $\mu<1 /(n+1), \vec{B}^{n}(\mu)$ by

$$
\bar{B}^{n}(\mu)=\left\{x \in T^{n} \mid \min _{i \in I_{n+1}} x_{i}=\mu\right\}
$$

THEOREM 5.7.1. Let $\mathrm{f}$ be a continuous function on $\mathrm{T}^{\mathrm{n}}$ and let $\mathrm{v}$ be the starting point. Assume there exist numbers $\mu<\min _{i} v_{i}$ and $\varepsilon>0$ such that for all $x \in \bar{B}^{-n}(\mu)$ there exists an index $i \in I_{n+1}$ such that

$$
x_{i}<v_{i} \text { and } f_{j}(x)-x_{j}<f_{i}(x)-x_{i}-\varepsilon
$$

for at least one index $j \in I_{n+1}, j \neq i$. Then the variable dimension restart algorithm applied with integer labelling and using the T-triangulation terminates with a completely labelled simplex if the grid size is small enough.

PROOF. Let the grid size be so small that

$$
\text { (5.7.2) } \sup _{x, y \in \sigma} \max _{i \in I}\left|f_{i}(x)-x_{i}-f_{i}(y)+y_{i}\right|<\frac{1}{2} \varepsilon
$$

for every simplex $\sigma$ such that $\sigma \cap \mathrm{B}^{-\mathrm{n}}(\mu) \neq \varnothing$. For some $T$, let $\sigma\left(\mathrm{w}^{1}, \ldots, \mathrm{w}^{\mathrm{t}+1}\right)$ be a t-dimensional simplex in $A(T)$ having points in common with $\bar{B}^{\mathrm{n}}(\mu)$. Then for any point $x \in \bar{B}^{n}(\mu) \cap \sigma\left(w^{1}, \ldots, w^{t+1}\right)$ there is an index $i$ such that (5.7.1) is satisfied for some index $j \neq i$. Hence with $(5.7 .2)$ it follows that

$$
f_{j}\left(w^{k}\right)-w_{j}^{k}<f_{i}\left(w^{k}\right)-w_{i}^{k} \quad k=1, \ldots, t+1 .
$$

So $\ell\left(w^{k}\right) \neq i$ for all $k$. Moreover, since $x_{i}<v_{i}$ we must have that $i \in T$. Consequently the simplex $\sigma$ cannot be generated by the algorithm since if so, $i \in T$ implies that at least one vertex $w^{k}$ must have label $i$. Hence the algorithm can only generate simplices in the interior of $\operatorname{conv}\left(\bar{B}^{n}(\mu)\right)$. Since this set is compact, the number of simplices meeting this set is finite, which proves the theorem. 
Observe that since the $T$ triangulation is used, $x_{i}<v_{i}$ implies $i \epsilon T$. In general this holds for any triangulation such that the diagonal

elements of the triangulation matrix are negative and the off-diagonal elements are nonnegative.

The condition of theorem 5.7.1 depends on the starting point v. Hence it is not sure that the condition is again satisfied if the algorithm is restarted with the last found approximation as the new starting point. Conditions to guarantee that at any stage a completely labelled simplex is found are given in the next theorem.

THEOREM 5.7.2. Let $f$ be a continuous function on $T^{n}$. Assume there exist numbers $\mu<1 /(n+1)$ and $\varepsilon>0$ such that for all $x \in \bar{B}^{-n}(\mu)$ we have that for at least one index $i$ with $x_{i}=\mu$,

$$
f_{j}(x)-x_{j}<f_{i}(x)-x_{i}-\varepsilon
$$

for at least one index $j \neq i$. Then, forr any starting point $v$ in the interior of $\operatorname{conv}\left(\bar{B}^{n}(\mu)\right)$, the variable dimension restart algorithm applied with integer labelling and using the $T$ triangulation terminates with a completely labelled simplex if the grid size is small encugh.

PROOF. Let the grid size be so small that

$$
\sup _{x, y \in \sigma} \max _{i \in I_{n+1}}\left|f_{i}(x)-x_{i}-f_{i}(y)+y_{i}\right|<\frac{1}{2} \varepsilon
$$

for every simplex $\sigma$ such that $\sigma \cap \bar{B}^{\mathrm{n}}(\mu)$ is nonempty. Then the proof of theorem 5.7.1 holds by noting that the condition of theorem 5.7.1. is implied by the condition of theorem 5.7.2.

Clearly if theorem 5.7.2 holds the algorithm terminates at any stage if

a) the grid size in the first stage is small enough and the sequence of grid sizes is decreasing

b) the starting point in the first stage is chosen in the interior of the convex hull of $\bar{B}^{-n}(\mu)$

c) the starting point in any other stage is chosen within the completely labelled simplex found in the previous stage. 
In the next theorem a condition is given such that the algorithm converges for every grid size and for any starting point.

THEOREM 5.7.3. Let $f$ be a continuous function on $T^{\mathrm{n}}$. Assume that there exists a number $\mu<1 /(n+1)$ such that for all $x$ with $x_{i}<\mu$ for at least one index $i \in I_{n+1}$, there exists an index $j \notin K$ such that

$$
f_{j}(x)-x_{j}<f_{h}(x)-x_{h} \text { for all } h \in K,
$$

where $\mathrm{K}$ is the set of indices $i$ with $x_{i}<\mu$. Then the variable dimension restart algorithm applied with integer labelling and using the $T$ triangulation terminates with a completely labelled simplex for every grid size and for any starting point.

PROOF. Let $\beta$ be the mesh of the triangulation. Define $\mu^{\prime}$ by

$$
\mu^{\prime}=\min \left(\mu, \min _{i \in I_{n+1}} v_{i}\right)-2 \beta
$$

Let $\sigma\left(w^{1}, \ldots, w^{t+1}\right)$ be a t-simplex of $A(T)$ meeting $\bar{B}^{n}\left(\mu^{\prime}\right)$. Then there is an index $h$ such that $x_{h} \leq \mu^{\prime}+\beta$ for all $x \in \sigma$. Hence $w_{h}^{i} \leq \mu^{\prime}+\beta<\mu$ for all $i=1, \ldots, t+1$. Consequently, using the assumptions of the theorem, $\ell\left(w^{i}\right) \neq h$ for all $i$. Moreover we have that $x_{h} \leq \mu^{\prime}+\beta<v_{h}$ for all $x \in \sigma$ which implies that $h \in T$. Therefore the simplex $\sigma$ cannot be generated by the algorithm, which proves that only simplices in the interior of $\operatorname{conv}\left(\bar{B}^{\mathrm{n}}\left(\mu^{\prime}\right)\right)$ are generated.

Analogous theorems can be given if vector labelling is used. We only give a theorem analogously to theorem 5.7.1. Theorems similar to the theorems 5.7 .2 and 5.7 .3 can be easily formulated.

THEOREM 5.7.4. Let $\mathrm{f}$ be a continuous function on $\mathrm{T}^{\mathrm{n}}$ and let $\mathrm{v}$ be the starting point. Then the algorithm applied with vector labelling terminates under the same conditions as formulated in theorem 5.7.1.

PROOF. Let the grid size be so small that

(5.7.3). $\sup _{\max }\left|f_{i}(x)-x_{i}-f_{i}(y)+y_{i}\right|<\frac{1}{2} \varepsilon$ $x, y \in \sigma \quad i \in I_{n+1}$ 
for every simplex $\sigma$ such that $\sigma \cap \bar{B}^{\mathrm{n}}(\mu) \neq \varnothing$.

For some $T$, let $\sigma\left(w^{1}, \ldots, w^{t+1}\right)$ be a simplex in $A(T)$ such that $\sigma \cap \bar{B}^{-n}(\mu) \neq \emptyset$. Let $x \in \bar{B}^{-n}(\mu)$ n. Then from (5.7.1) and (5.7.3) it follows that there exists an index $i$ and an index $j \neq i$ such that $x_{i}<v_{i}$ and

$$
\bar{f}_{j}\left(w^{k}\right)-w_{j}^{k}<\bar{f}_{i}\left(w^{k}\right)-w_{i}^{k} \quad k=1, \ldots, t+1
$$

Hence $\bar{f}_{i}(y)-y_{i}>\bar{f}_{j}(y)-y_{j}$ for all $y \in \sigma\left(w^{1}, \ldots, w^{t+1}\right)$, where $\bar{f}$ is the piecewise linear approximation to $f$ with respect to the triangulation. As argued in the previous section the algorithm traces a path of points $y$ such that for some $\alpha(y)$

and

$$
\bar{f}_{h}(y)-y_{h}=-\alpha(y) \text { if } h \in T
$$

$$
\bar{f}_{h}(y)-y_{h}>-\alpha(y) \text { if } h \notin T \text {. }
$$

So if $\sigma\left(w^{1}, \ldots, w^{t+1}\right)$ is generated by the algorithm we must have that $i \notin 7$ since $\bar{f}_{i}(y)-y_{i}>\bar{f}_{j}(y)-y_{j}$ for all $y \in \sigma$. On the other hand we have $x_{i}<v_{i}$ implying that $i \in T$. Hence a contradiction is obtained and consequently $\sigma$ cannot be generated, which proves the theorem.

As argued in section 4.8, condition (5.7.3) cannot be guaranteed for any grid size if we deal with an u.s.c. mapping $\phi$ instead of a function.

Then we need e.g. that, for some $\mu<\min _{i} v_{i}$ and some $0<\delta<\min _{i} v_{i}-\mu$, (5.7.1) holds for any $y \in T^{n} \cap B^{n+1}(x, \delta)$ and for any $f(y) \in \phi(y)$.

Then the algorithm converges if the mesh of the $T$ triangulation is less than $\delta$.

In the next theorem a condition to guarantee the convergence of the algorithm in case of an u.s.c. mapping is stated, which is comparable with Merrill's condition given in lemma 2.6.2. Recall that in the theorems 5.7.1-5.7.4 there was a weak dependency of the proof on the underlying triangulation. In the proof of the next theorem this dependency is much stronger. In particular it will appear that the proof is not valid if the standard triangulation is used. 
THEOREM 5.7.5. Let $\phi$ be an u.s.c. mapping on $T^{n}$. For some $\delta>0$, let there exist $w \in T^{n}$ and $\mu<1 /(n+1)$ such that for all $x \in T^{n}$ with $\min x_{i}<\mu$ and all $\geqslant \epsilon T^{n} \cap B^{n+1}(x, \delta)$ holds $i \in I_{n+1}$

$$
(x-h(x))^{\top}(w-z)<0
$$

for all $\mathrm{h}(\mathrm{x}) \epsilon \phi(\mathrm{x})$. Then the variable dimension restart algorithm applied with vector labelling and using the $T$ triangulation terminates with a completely labelled simplex if the mesh of the triangulation is less then $\delta$.

PROOF. Let $\mathrm{v}$ be an arbitrarily chosen starting point. Define $\mu^{\prime}$ by

$$
\mu^{\prime}=\min \left\{\mu-\delta, \min _{i \in I_{n+1}} v_{i}+\min _{i \in I_{n+1}} n\left(v_{i}-w_{i}\right)\right\}
$$

For some $T$, let $\sigma\left(w^{1}, \ldots, w^{t+1}\right)$ be a $t$-simplex in $A(T)$ such that for some $y \in \sigma, \min _{i \in I_{n+1}} y_{i}<\mu^{\prime}$. We prove that the system of linear equations

$$
\sum_{i=1}^{t+1} \lambda_{i} l\left(w^{i}\right)+\sum_{h \notin T} \mu_{h} e(h)=e
$$

does not have a nonnegative solution $\lambda_{i}^{*} i=1, \ldots, t+1$ and $\mu_{h}^{*}, h \notin T$. Suppose there exists such a solution. Then

$$
\text { (5.7.4) (n+1) } \sum_{i=1}^{t+1} \lambda_{i}^{*}+\sum_{h \notin T} \mu_{h}^{*}=n+1 .
$$

Furthermore, we have that

$$
\text { (5.7.5) } \quad\left[\sum_{i=1}^{t+1} \lambda_{i}^{*} l\left(w^{i}\right)+\sum_{h \notin T} \mu_{h}^{*} e(h)\right]^{\top}(w-y)=e^{\top}(w-y)=0
$$

since $w, y \in T^{n}$. We obtain a contradiction to $(5.7 .5)$ by proving that $\ell\left(w^{i}\right)^{\top}(w-y)<0$ for all $i$ and $e(h)^{\top}(w-y)<0$ for all $h \notin T$ since by (5.7.4) at least one $\lambda_{i}^{*}$ or $\mu_{h}^{*}$ is positive. Let $f$ be the piecewise linear approximation to $\phi$ with respect to the triangulation. Clearly $f\left(w^{i}\right) \in \phi\left(w^{i}\right), i=1, \ldots, t+1$. Let $k$ be the index such that $y_{k}=\min _{i \in I_{n+1}} y_{i}$. Then $w_{k}^{i} \leq y_{k}+\delta<\mu^{\prime}+\delta \leq \mu$, which implies that 


$$
\ell\left(w^{i}\right)^{\top}(w-y)=\left(w^{i}-f\left(w^{i}\right)+e\right)^{\top}(w-y)=\left(w^{i}-f\left(w^{i}\right)\right)^{\top}(w-y)<0 \quad i=1, \ldots, t+1 .
$$

It remains to prove that $w_{h}-y_{h}<0$ for all $h \notin T$. First note that

$\min _{i \in I_{n+1}}\left(v_{i}-w_{i}\right) \leq 0$. Hence $y_{k}=\min _{i \in I_{n+1}} y_{i}<\mu^{\prime} \leq \min _{i \in I_{n+1}} v_{i}+\min _{i \in I_{n+1}} n\left(v_{i}-w_{i}\right) \leq v_{k}$ implying that

$k \in T$ by the structure of the $T$ triangulation. Moreover it follows that

$$
\mathrm{y}_{\mathrm{k}}<\mathrm{v}_{\mathrm{k}}+\mathrm{n}\left(\mathrm{v}_{\mathrm{h}}-\mathrm{w}_{\mathrm{h}}\right) \quad \text { for all } \mathrm{h} \notin T
$$

whereas, again by the structure of the $T$ triangulation

$$
\mathrm{y}_{\mathrm{h}} \geq \mathrm{v}_{\mathrm{h}}+\mathrm{n}^{-1}\left(\mathrm{v}_{\mathrm{k}}-\mathrm{y}_{\mathrm{k}}\right) \text { for all } \mathrm{h} \notin T
$$

Both inequalities together give

$$
y_{h}>v_{h}-\left(v_{h}-w_{h}\right)=w_{h} \quad \text { for all } h \notin T \text {, }
$$

and a contradiction to (5.7.5) is obtained. Hence the algorithm can only generate simplices in $\operatorname{conv}\left(\bar{B}^{n}\left(\mu^{\prime}\right)\right)$.

Observe that (5.7.6) does not hold if the standard triangulation is used. Only in the case that $v=w$ we obtain immediately $y_{h} \geq_{h} \cdot h \notin T$, which is sufficient to obtain a contradiction to (5.7.5). On the other hand the theorem can be proved for any triangulation such that the diagonal elements of the matrix are negative and the off diagonal elements positive, by taking $\mu^{\prime}$ large enough.

Clearly the condition "for some $\delta$ " can be strengthened to "for all $\delta "$. Then the algorithm converges for every grid size.

\subsection{COMPUTATIONAL RESULTS.}

The variable dimension restart algorithm is applied for vector labelling to three examples in which the equilibrium price vector in a pure exchange economy has to be computed. The data of these problems can be found in Scarf [1967b]. The algorithm is applied with the standard triangulation as well as the $T$ triangulation. However, in stead of the 
matrix $Q(T)$ we use the matrix $-O(-T)$. In addition, a point $x$ outside the interior of $s^{n}$ receives the label $e(i)$ with $i$ the smallest index such that $x_{i} \leq 0$, whereas a point $x \in$ int $S^{n}$ receives the label $\ell(x)$ defined by

$$
\ell_{j}(x)=g_{j}(x) / a_{j} \quad j=1, \ldots, n+1,
$$

where $g_{j}(x)$ is the total demand of good $j$, given that $x$ is the price vector, and $a_{j}$ is the total supply of good $j$. It can easily be seen that a completely labelled simplex yields a good approximation of a fixed point. Let $\mathrm{w}^{*}(\mathrm{~m})$ be the approximate fixed point in stage $\mathrm{m}$, i.e.

$$
\mathrm{w}^{*}(\mathrm{~m})=\sum_{i=1}^{\mathrm{n}+1} \lambda_{i}^{\mathrm{m}} \mathrm{w}^{\mathrm{i}}(\mathrm{m})
$$

where $\mathrm{w}^{1}(\mathrm{~m}), \ldots, \mathrm{w}^{\mathrm{n}+1}(\mathrm{~m})$ are the vertices of the completely labelled simplex generated by the algorithm in stage $m$ and $\lambda_{1}^{m}, \ldots, \lambda_{n+1}^{m}$ the corresponding solution to the system of linear equations. Then the starting point in stage $\mathrm{m}+1$ is $\mathrm{w}^{*}(\mathrm{~m})$, whereas the starting point in the first stage is $w^{*}(0)$ defined by $w_{i}^{*}(0)=(n+1)^{-1}, i \in I_{n+1}$. Finally we have to make a remark about the sequence of grid sizes. Let $h_{m}$ be defined by

$$
h_{m}=\max _{i \in I_{n+1}}\left(w_{i}^{*}(m)-w_{i}^{*}(m-1)\right) \quad m=1,2, \ldots
$$

and let $\delta_{m}$ and $G_{m}$ be the grid size and the triangulation in stage $m$. Then

$$
\begin{array}{ll}
\delta_{m+1}=h_{m} \delta_{m} \sqrt{n+1} / \text { mesh } G_{m} & \text { if } h_{m}<\left(\text { mesh } G_{m}\right) / 2 \sqrt{n+1} \\
\delta_{m+1}=\frac{1}{2} \delta_{m} & \text { if } h_{m} \geq\left(\operatorname{mesh} G_{m}\right) / 2 \sqrt{n+1} .
\end{array}
$$

The results are given in the tables 5.8.1-5.8.3. In all examples the number of function evaluations is equal to the number of linear programming steps, i.e. the dimension is never decreased. 
Table 5.8.1. Pure exchange economy, $n=4, M=\left(\right.$ mesh $\left.G_{m}\right) / 2 \sqrt{n+1}, N$ is the cumulative number of iterations, $E=\max _{i \in I}\left|\left(g_{i}-a_{i}\right) / a_{i}\right|$.

\begin{tabular}{|c|c|c|c|c|c|c|}
\hline & \multicolumn{3}{|c|}{ T-triangulation } & \multicolumn{3}{|c|}{ Standard triangulation } \\
\hline Stage & M & $\mathrm{N}$ & $E$ & M & $\mathrm{N}$ & $E$ \\
\hline 1 & .051 & 7 & 1.02 & .093 & 8 & 1) \\
\hline 2 & .026 & 13 & .18 & .047 & 16 & .21 \\
\hline 3 & .013 & 18 & .09 & .023 & 26 & .15 \\
\hline 4 & .006 & 24 & .04 & .012 & 31 & .05 \\
\hline 5 & $1.210^{-3}$ & 30 & $210^{-3}$ & $5.410^{-3}$ & 36 & $10^{-2}$ \\
\hline 6 & $6.110^{-4}$ & 36 & $810^{-5}$ & $1.010^{-3}$ & 47 & $410^{-4}$ \\
\hline 7 & $3.810^{-5}$ & 43 & $210^{-7}$ & $2.210^{-4}$ & 54 & $310^{-6}$ \\
\hline 8 & $1.510^{-6}$ & 50 & $310^{-11}$ & $8.410^{-6}$ & 63 & $10^{-9}$ \\
\hline
\end{tabular}

1) $w^{*}(1) \notin \operatorname{int} s^{n}$.

Table 5.8.2. Pure exchange economy, $n=7, M=\left(\operatorname{mesh} G_{m}\right) / 2 \sqrt{n+1}, N$ is the cumulative number of iterations, $E=\max _{i \in I}\left|\left(g_{i}-a_{i}\right) / a_{i}\right|$.

$$
i \in I_{n+1}
$$

\begin{tabular}{|c|c|c|c|c|c|c|}
\hline & \multicolumn{3}{|c|}{ T-triangulation } & \multirow{2}{*}{$\begin{array}{l}\text { Standard } \\
\mathrm{M}\end{array}$} & \multicolumn{2}{|c|}{ triangulation } \\
\hline Stage & M & $\mathrm{N}$ & $\mathrm{E}$ & & $\mathrm{N}$ & $E$ \\
\hline 1 & .032 & 11 & 1.32 & .055 & 9 & .89 \\
\hline 2 & .016 & 20 & .22 & .019 & 38 & .26 \\
\hline 3 & .008 & 28 & .12 & .009 & 49 & .09 \\
\hline 4 & $3.210^{-3}$ & 42 & .03 & $4.210^{-3}$ & 66 & .02 \\
\hline 5 & $1.610^{-3}$ & 59 & $510^{-3}$ & $1.210^{-3}$ & 79 & $10^{-3}$ \\
\hline 6 & $4.110^{-4}$ & 79 & $210^{-4}$ & $2.710^{-4}$ & 95 & $210^{-5}$ \\
\hline 7 & $7.210^{-5}$ & 100 & $210^{-6}$ & $1.810^{-5}$ & 111 & $310^{-8}$ \\
\hline 8 & $3.210^{-6}$ & 121 & $810^{-10}$ & $3.210^{-7}$ & 127 & $<10^{-13}$ \\
\hline
\end{tabular}


Table 5.8.3. Pure exchange economy, $n=9, M=\left(\right.$ mesh $\left.G_{m}\right) / 2 \sqrt{n+1}, N$ is the cumulative number of iterations, $E=\max _{i \in I}\left|\left(g_{i}-a_{i}\right) / a_{i}\right| \cdot$

\begin{tabular}{|c|c|c|c|c|c|c|}
\hline & \multicolumn{3}{|c|}{ T triangulation } & \multicolumn{3}{|c|}{ Standard triangulation } \\
\hline Stage & M & $\mathrm{N}$ & $E$ & M & $\mathrm{N}$ & $E$ \\
\hline 1 & .025 & 12 & .30 & .045 & 10 & .59 \\
\hline 2 & .013 & 26 & .18 & .011 & 65 & .04 \\
\hline 3 & .006 & 42 & .06 & .006 & 84 & .01 \\
\hline 4 & .003 & 58 & .01 & $8.710^{-4}$ & 110 & $310^{-4}$ \\
\hline 5 & $8.710^{-4}$ & 75 & $810^{-4}$ & $2.110^{-4}$ & 136 & $410^{-6}$ \\
\hline 6 & $2.110^{-4}$ & 91 & $10^{-5}$ & $6.310^{-6}$ & 169 & $10^{-9}$ \\
\hline 7 & $1.410^{-5}$ & 108 & $210^{-8}$ & $7.010^{-8}$ & 205 & $<10^{-13}$ \\
\hline 8 & $2.510^{-7}$ & 124 & $<10^{-13}$ & & & \\
\hline
\end{tabular}


CHAPTER 6

VARIABLE DIMENSION RESTART ALGORITHM ON $\mathrm{R}^{\mathrm{n}}$

\subsection{INTRODUCTION.}

In this chapter we discuss the application of the variable dimension restart algorithm to compute a fixed point of a function or mapping from $\mathrm{R}^{\mathrm{n}}$ into itself. This chapter is based on Van der Laan and Talman [1979b,1980c]. Again the algorithm starts with a zero-dimensional simplex and generates a path of adjacent $t$-dimensional simplices, $0 \leq t \leq n$. This path either terminates with an n-dimensional simplex yielding a good approximation of a fixed point or goes to infinity. Before we treat the application on the unbounded region $\mathrm{R}^{\mathrm{n}}$, we first describe in section 2 the application of the algorithm on the unit cube.

Conditions for the convergence of the method on $R^{n}$ are given in section 3 . Further research about the variable dimension algorithm is summarized in section 4. Computational results are given in section 5 .

\subsection{THE UNIT CUBE.}

To approximate a fixed point of a function or mapping on the n-dimensional unit cube $C^{n}=\left\{x \in R^{n}, 0 \leq x_{i} \leq 1, i=1, \ldots, n\right\}$, this set is triangulated by the $\mathrm{K}$ triangulation with grid size $\mathrm{m}^{-1}$, where $\mathrm{m}$ is a positive integer, and $z=0$ (see definition 3.2.1). We first describe the algorithm for integer labelling. We call a labelling rule proper if $\ell(x) \neq i$ if $x_{i}=1$ and $\ell(x) \neq n+1$ if $x_{i}=0$ for some index $i \in I_{n+1}$. The standard integer labelling on $R^{n}$ defined in definition 4.2 .3 induces a labelling on $\mathrm{C}^{\mathrm{n}}$. We now take this labelling rule and assume it is a proper labelling rule as is the case when $\mathrm{f}$ is a function from $\mathrm{C}^{\mathrm{n}}$ into the interior of $\mathrm{C}^{\mathrm{n}}$. The triangulation matrix corresponding to the $\mathrm{K}$ triangulation is the $\mathrm{n} \times \mathrm{n}$ identity matrix. To apply the variable dimension restart algorithm we define the $n \times(n+1)$ matrix $U$ by $U=(I,-e)$. So $u(i)=e(i), i=1, \ldots, n$ and 
$u(n+1)=-e=-\sum_{i=1}^{n} u(i)$, where $u(i)$ is the $i-t h$ column of $u$. As in section 5.2 $u(i)$ corresponds to the $i$-th direction in which a search is to be made if label $i$ has been found. For a given subset $T$ of $I_{n+1},|T| \leq n$, the $t$-simplex $\sigma\left(\mathrm{y}^{1}, \pi(T)\right)$ is the simplex with vertices $\mathrm{y}^{1}, \ldots, \mathrm{y}^{\mathrm{t+1}}$ such that $\mathrm{y}^{1}$ is a grid point, $\pi(T)$ a permutation of the elements of $T$ and $y^{i+1}=y^{i}+u(i) / m$, $i=1, \ldots, t$. Observe that such a simplex is indeed a face of a simplex of the $\mathrm{K}$ triangulation. Again a $(t-1)$-simplex $\sigma$ is called $T$-complete if the $t$ vertices of $\sigma$ carry all the labels of the set $T$.

Let the grid point $\mathrm{v}$ be an arbitrarily chosen starting point. Then the variable dimension restart algorithm starts with the zero dimensional simplex $\{\mathrm{v}\}$ and proceeds according to the steps $0-4$ of section 5.2 except that the columns of the matrix $Q$ in table 5.2 .1 must be changed into the columns of $U$. Again we can give an intexpretation of the method by defining the region $A(T)$ as

$$
A(T)=\left\{x \in C^{n} \mid x=v+\sum_{j \in T} \lambda_{j} u(j) \text { for nonnegative } \lambda_{j}, j \in T\right\},
$$

where $T$ is a proper subset of $I_{n+1}$. Then we have that a path of simplices of variable dimension is generated such that the common facet of two adjacent $t$-simplices in $A(T)$ is $T$-complete. Moreover, the dimension is increased $i f$, for some $j \notin T$, a $(T \cup\{j\})$-complete simplex in $A(T)$ is generated whereas the dimension is decreased if, for some $k \in T$, a simplex in $A(T)$ is generated having a $T$-complete facet in $A(T\{k\})$. In the first case the algorithm continues with a simplex in $A(T \cup\{j\})$, whereas in the latter case the method proceeds with a simplex in $A(T \backslash\{k\})$. The algorithm is illustrated in figure 6.2 .1 for $n=2$ and $m=7$. In this example we have that $\tau_{1}$ is a $\{1,2\}$-complete one-simplex of $A(1)$. So the dimension is increased and the label of the new vertex $u$ is calculated. The one-simplex $\tau_{2}$ is an $\{1,2\}$-complete facet of $\sigma_{1}$ in $A(\{1,2\} \backslash\{1\})$. The dimension is decreased and the vertex $w$ having label 1 is replaced. The simplex $\tau_{3}$ is a $\{2,3\}$-complete facet in $A(2)$ and the dimension is again increased. Then the algorithm continues with 2 -simplices in $\mathrm{A}(2,3)$ until the completely labelled simplex $\sigma_{2}$ is found. 


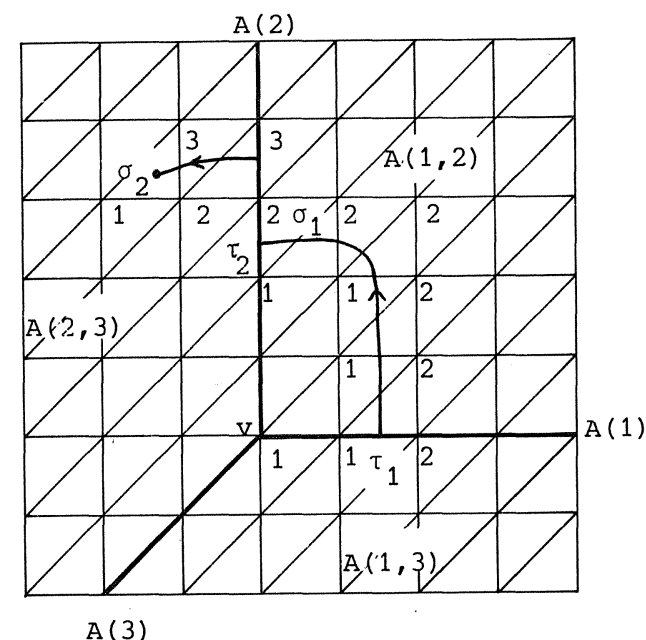

Figure 6.2.1. Illustration of the variable dimension restart algorithm on $c^{n}, n=2, m=7, v=(3 / 7,2 / 7)^{\top}$.

The proof that the algorithm can never return in a simplex is identical to the proof given in section 5.4. Therefore the algorithm finds always a completely labelled simplex if all steps are feasible. First we show that a replacement step is always feasible. Let $\sigma\left(y^{1}, \ldots, y^{t+1}\right)$ be a t-simplex in $A(T)$ generated by the algorithm and let $y^{s}$ be the vertex of $\sigma$ to be replaced. Clearly this replacement step is feasible except when for some $j(1 \leq j \leq n)$ either $y_{j}^{s}=m^{-1}$ and $y_{j}^{i}=0, i \neq s$, or $y_{j}^{s}=1-m^{-1}$ and $y_{j}^{i}=1, i \neq s$. Suppose the first case is true. If $v_{j}>0$, we must have that $n+1$ is an element of $T$ otherwise the $j$-th component could not have been decreased. However by the proper labelling $\ell\left(y^{i}\right) \neq n+1$ for ail $i \neq s$, whereas $y^{S}$ has to be replaced iff the facet with vertices $y^{i}, i \neq s$, is $T$-complete. So a contradiction is obtained. This proves that if $y^{S}$ has to be replaced there exists an index $i, i \neq s$, such that $y_{j}^{i}>0$. Hence the replacement step is feasible.

If $v_{j}=0$ then either $n+1 \epsilon T$ which is identical to the case just mentioned, or $n+1 \notin T$. Then, however, $s=t+1$ and $R_{\pi_{t}}=0$ implying that the dimension should be decreased and contradicting the fact that $\mathrm{y}^{\mathbf{S}}$ must be replaced according to table 5.2.1.

Suppose now that $y_{j}^{i}=1$ for all $i \neq s$. If $v_{j}<1$ we must have $j \in T$. Again because of the proper labelling $\ell\left(y^{i}\right) \neq j$ for all $i \neq s$. Hence, if $y^{S}$ has to be 
replaced there exists an index $i$ such that $\mathrm{y}_{j}^{i<1}$ implying that the replacement step is feasible. If $v_{j}=1$, either $j \in T$ and then there is an index $i$ with $y_{j}^{i}<1$, or $j \notin T$ again implying that the dimension should be decreased. So all replacement steps are feasible.

Next we prove that the extension from a $t$ to a $(t+1)$-simplex, occurring when a new label is found, is also feasible. So, let $\sigma\left(y^{1}, \pi(T)\right)$ be a $(T \cup\{j\})-$ complete simplex in $A(T)$, generated by the algorithm. Since $j \notin T$ we have that $\pi_{i} \neq j, i=1, \ldots, t$. Moreover we have that for some vertex $y^{s}, \ell\left(y^{s}\right)=j$. If $j=n+1$ then because of the proper labelling $y_{h}^{s} \geq m^{-1}$ for all $h$. Hence for all $i \geq s, y_{h}^{i} \geq m^{-1}$ for all $h$, since $\pi_{i} \neq n+1$ for all $i$. Consequently, $0 \leq y_{h}^{t+2}=y_{h}^{t+1}-$ $\mathrm{m}^{-1} \leq 1$ for all $\mathrm{h}$, i.e. $\mathrm{y}^{\mathrm{t}+2}$ is a point in $\mathrm{c}^{\mathrm{n}}$. If $j \neq n+1$ then again because of the proper labelling $y_{j}^{s} \leq 1-m^{-1}$. This implies that $y_{j}^{t+1} \leq 1-m^{-1}$, since $\pi_{i} \neq j, i \geq s$. Hence $1 \geq y_{j}^{t+2}=y_{j}^{t+1}+m^{-1} \geq 0$. Moreover $y_{h}^{t+2}=y_{h}^{t+1}$ for all $h \neq j$, so that $y^{t+2}$ is a point in $\mathrm{C}^{\mathrm{n}}$. Consequently all steps of the algorithm are feasible.

We now discuss for vector labelling the application of the algorithm to approximate a fixed point of an u.s.c. mapping $\phi$. Each point $x$ of $c^{n}$ receives a vector label $l(x)$ based on the rule given in definition 4.8.1. Taking $b=e, l$ is a function from $C^{n}$ to $R^{n+1}$ with

and

$$
\ell_{i}(x)=f_{i}(x)-x_{i}+1 \quad i=1, \ldots, n
$$

$$
\ell_{n+1}(x)=1 \text {, }
$$

where $f(x)$ is a piecewise linear approximation to the mapping $\phi$ with respect to the triangulation of $\mathrm{C}^{\mathrm{n}}$. Analogously to theorem $5.6 .1 \mathrm{a}$ $(t-1)$-simplex $\sigma\left(\mathrm{w}^{1}, \ldots, \mathrm{w}^{t}\right)$ is called $T$-complete if the system of $\mathrm{n}+1$ linear equations

$$
\sum_{i=1}^{t} \lambda_{i} l\left(w^{i}\right)+\sum_{h \notin T} \mu_{h} e(h)=e
$$

has a nonnegative solution $\lambda_{i}^{*}, i=1, \ldots, t$ and $\mu_{h}^{*}, h \notin T$. In the following we assume that degeneracy does not occur. The next corollary follows immediately by considering the $(n+1)$-th equation of the system of linear equations.

COROLLARY 6.2.1. For some $T$, let $\sigma\left(w^{1}, \ldots, w^{t}\right)$ be a $T$-complete simplex with $\lambda_{i^{\prime}}^{*} i=1, \ldots, t$ and $\mu_{h^{\prime}}^{*} h \notin T$ the solution of the system of linear equations. If $n+1 \notin T$, then 


$$
\sum_{i=1}^{t} \lambda_{i}^{*}+\mu_{n+1}^{*}=1 .
$$

If $n+1 \in T$, then

$$
\sum_{i=1}^{t} \lambda_{i}^{*}=1
$$

From thiscorollary it follows immediately that $\sum_{i=1}^{n+1} \lambda_{i}^{*}=1$ if $\sigma\left(w^{1}, \ldots, w^{n+1}\right)$ is completely labelled,i.e. if $\sigma$ is an $I_{n+1}$-complete simplex. Then $x^{*}=\sum_{i=1}^{n+1} \lambda_{i}^{*} w^{i}$ is a good approximation of a fixed point (see lemma 4.8.3).

The steps of the algorithm are identical to those of section 5.6 except that the matrix $U$ must be used in stead of the matrix $Q$. So, starting with an arbitrarily chosen grid point $v \in C^{n}$, the algorithm generates a path of adjacent simplices. As in section 5.6 a simplex can never be revisited, so a completely labelled $n$-simplex must be found provided that the steps are feasible. Since $\mathrm{C}$ is bounded, all linear programming steps are feasible. Concerning the other steps we fixst prove that the extension to a higher dimensional simplex is always feasible. For some $T$, let $\sigma\left(y^{1}, \pi(T)\right.$ be a $(T \cup\{h\})$-simplex in $A(T)$ generated by the algorithm. First consider the case where $h=n+1$. Then $e(n+1)$ is eliminated by the last linear programming step and it follows from corollary 6.2.1 that $\sum_{i=1}^{t+1} \lambda_{i}^{*}=1$. We shall now verify that $y_{j}^{t+1}>0$ for all $j$. To do this, suppose to the contrary that $y_{j}^{t+1}=0$ for some $j$. Since $n+1 \notin T$, we have that $\pi_{i} \neq n+1$, $i=1, \ldots, t$. Hence $y_{j}^{i}=0$ for all $i$, implying that $\pi_{i} \neq j, 1 \leq i \leq t$. So $j \notin T$ and the $j-$ th equation of the system of linear equations is

$$
\sum_{i=1}^{t+1} \lambda_{i}^{*} j^{k}\left(y^{i}\right)+\mu_{j}^{*}=1
$$

with $\mu_{j}^{*}>0$. Since $y_{j}^{i}=0, \ell_{j}\left(y^{i}\right) \geq 1$ for all $i$. Consequently $\sum_{i=1}^{t+1} \lambda_{i}^{*}<1$ which contradicts that $\sum_{i=1}^{t+1} \lambda_{i}^{*}=1$. Hence, if $h=n+1, y_{j}^{t+1}>0$ for $a l 1 j$ and so $y^{t+2}=$ $y^{t+1}+u(n+1) / m=y^{t+1}-e / m \geq 0$.

Next we prove that the extension is feasible if $h \neq n+1$. Now we verify that $y_{h}^{t+1}<1$. Again we suppose to the contrary that $y_{h}^{t+1}=1$. Since $h \notin T, \pi_{i} \neq h$ for $i=1, \ldots, t$ implying that $y_{h}^{i}=1$ for all $i$ and therefore $n+1 \notin T$. So from corollary 6.2 .1 we obtain that $\sum_{i=1}^{t+1} \lambda_{i}^{*}=1-\mu_{n+1}^{*}<1$. However, since $\sigma$ is $(T \cup\{h\})$-complete, $e(h)$ is eliminated and the $h$-th equation is 


$$
\sum_{i=1}^{t+1} \lambda_{i}^{*} l_{h}\left(y^{i}\right)=1
$$

Since $f\left(y^{i}\right) \in C^{n}, \ell_{h}\left(y^{i}\right) \leq 1, i=1, \ldots, t+1$. Hence $\sum_{i=1}^{t+1} \lambda_{i}^{*} \geq 1$, contradicting the assumption that $y_{h}^{t+1}=1$. Consequently $y_{h}^{t+1}<1$ and so $y^{t+2}=y^{t+1}+e(h) / m$ is a point in $\mathrm{C}^{\mathrm{n}}$.

To prove that all replacement steps are feasible we assume that piecewise linear approximation $\mathrm{f}$ to $\phi$ is a continuous function from $\mathrm{C}^{\mathrm{n}}$ into the interior of $\mathrm{C}^{\mathrm{n}}$. For some $T$, let $\sigma\left(\mathrm{y}^{1}, \ldots, \mathrm{y}^{t+1}\right)$ be a $t$-simplex in $A(T)$ generated by the algorithm and let $\mathrm{y}^{\mathbf{S}}$ be the vertex to be replaced according to table 5.2.1. This step is feasible unless either

or

a) for some $j, y_{j}^{i}=0$ for all $i \neq s$ and $y_{j}^{s}=m^{-1}$

b) for some $j, y_{j}^{i}=1$ for all $i \neq s$ and $y_{j}^{s}=1-m^{-1}$.

We prove that in both cases the system of linear equations

$$
\sum_{i \neq s} \lambda_{i} l\left(y^{i}\right)+\sum_{h \notin T} \mu_{h} e(h)=e
$$

has no feasible solution. Suppose to the contrary that the system has a feasible solution $\lambda_{i}^{*}$, iłs and $\mu_{h}^{*} h \notin T$. Consider case a) with $v_{j}>0$. Then $n+1 \epsilon T$ and so by corollary $6.2 .1 \sum_{i \neq s} \lambda_{i}^{*}=1$. The $j$-th equation of the system $(6.2 .1)$ is given by

$$
\sum_{i \neq s} \lambda_{i}^{*} l_{j}\left(y^{i}\right)+\bar{\mu}_{j}=1
$$

with $\bar{\mu}_{j}=\mu_{j}^{*}$ if $j \notin T$ and $\bar{\mu}_{j}=0$ if $j \in T$. Since $f$ is a function from $c^{n}$ into the interior of $c^{n}$ we have that $\ell_{j}\left(y^{i}\right)=f_{j}\left(y^{i}\right)-y_{j}^{i}+1>1$. Hence from (6.2.2) it follows that $\Sigma_{i \neq s} \lambda_{i}^{*}<1$ which contradicts $\Sigma_{i \neq s} \lambda_{i}^{*}=1$. So there is no feasible solution if $v_{j}>0$. Consider now the case with $v_{j}=0$. If $n+1 \in T$, we can repeat the above arguments. If $n+1 \notin T$ it follows that $s=t+1$ and $\pi_{t}=j$. Hence $R_{\pi_{t}}=R_{j}=y_{j}^{1}-v_{j}=0$, implying that step 4 should be performed.

In case b) we distinguish between $v_{j}<1$ and $v_{j}=1$. If $v_{j}<1$ we have that $j \in T$. Hence the $j$-th equation of $(6.2 .1)$ is 


$$
\sum_{i \neq s} \lambda_{i} l_{j}\left(y^{i}\right)=1
$$

with $\ell_{j}\left(y^{i}\right)<1$ for all $i \neq s$ implying that $\sum_{i \neq s} \lambda_{i}^{*}>1$.

However from corollary 6.2 .1 it follows that $\Sigma_{i \neq s} \lambda_{i}^{*} \leq 1$, which again gives a contradiction. Finally if $v_{j}=1$ we have that, either $j \in T$ and we can

repeat the arguments as for $v_{j}<1$, or $s=t+1$ and $R_{\pi_{t}}=0$, implying that step 4 should be performed. Hence, if $\mathrm{f}$ is a function from $\mathrm{C}^{\mathrm{n}}$ into the interior of $C^{n}$, all replacement steps are feasible and the algorithm terminates with a completely labelled simplex within a finite number of iterations.

\subsection{CONVERGENCE CONDITIONS ON $\mathrm{R}^{\mathrm{n}}$.}

In this section the variable dimension restart algorithm is applied to compute a fixed point of a function or mapping on $\mathrm{R}^{\mathrm{n}}$. Conditions are given to guarantee that the algorithm converges. For some positive $\delta, R^{n}$ is triangulated by $\mathrm{AK}_{\delta}$ where $\mathrm{A}$ is a triangulation matrix (c.f. section 3.3), such that the arbitrarily or by priory information chosen starting point $v$ is a grid point of the triangulation. It seems to be adequate to use an element of the subclass $A$ of $A K$ triangulations in fixed point algorithms. Adding the i-th column of a matrix of the form $A(\alpha)$ to a point $y$ of $R^{n}$ means that only the $i$-th component of $y$ is increased, whereas all other components are affected by the same amount. So in going from $y^{1}$ to $y^{n+1}$ where $y^{i}, i=1, \ldots, n+1$, are the vertices of a simplex $\sigma\left(y^{1}, \pi\left(I_{n}\right)\right)$ of the triangulation, all components have been increased by the same amount. Note that in all the algorithms discussed in chapter 4 only the columns of the triangulation matrix (if $\mathrm{AK}$ is the underiying triangulation) appear in the rules for the replacement steps. However, the variable dimension restart algorithm uses the extra column $a(n+1)=-\sum_{i=1}^{n} a(i)$. So using an $A(\alpha) K_{\delta}$ triangulation a search in the $i-t h$ direction, $i \leq n$, means that the $i$-th component is increased and all other components are decreased with the same amount, whereas a search in the $(n+1)$-th direction means that all components are decreased with the same amount. In section 3.3 we summarized some nice properties of the $A(n+\sqrt{n+1}) K_{\delta}$ triangulation. These properties suggested that it is preferable to use this element of the subclass A. The application of the variable dimension restart algorithm provides an extra argument. Recall that the regions $A(T)$ depend on the underlying triangulation. So, using the $A(n+\sqrt{n+1}) K_{\delta}$ triangulation the 
$n$-dimensional real space $R^{n}$ is subdivided in subsets $A(T), T \subset I_{n+1}$ and $|T| \leq n$, defined by

$$
A(T)=\left\{x \in R^{n} \mid x=v+\sum_{j \in T} \lambda_{j} a(j) \text { for nonnegative } \lambda_{j}, j \in T\right\} \text {, }
$$

where $a(i)$ is the $i$-th column of the $n \times(n+1)$ matrix $A(n+\sqrt{n+1)}$ defined by

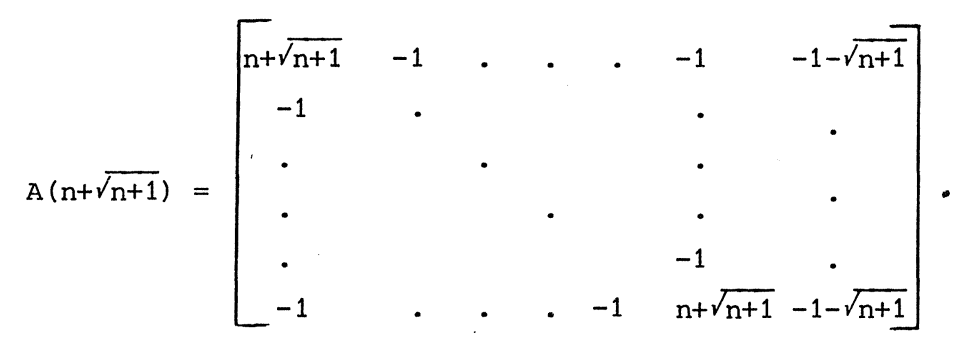

Consider the $n$-dimensional subsets $A\left(T_{k}\right)$ with $\left.T_{k}=I+1 \backslash k\right\}, k=1, \ldots, n+1$. Then the $A(n+\sqrt{n+1}) K_{\delta}$ triangulation has the property that it divides $R^{n}$ in $n+1$ n-dimensional subsets $A\left(T_{k}\right)$ of "equal size". With subsets of equal size we mean the following. Recall that $B^{n}(v, \mu)$ is a ball with $v$ as centre point and $\mu$ as length of the radius. Then for any (positive) $\mu$ the volume of the intersection of $B^{n}(v, \mu)$ and $A\left(T_{k}\right)$ is equal for all $k \in I_{n+1}$. This property follows immediately from the fact that, as pointed out by Todd [1978f], the angle between two half lines $v+\lambda a(k), \lambda \geq 0$, is equal for any two indices $\mathrm{k}_{1}, \mathrm{k}_{2}$. So within a ball with the starting point $\mathrm{v}$ as centre point the number of $n$ simplices in $A\left(T_{k}\right)$ is roughly the same for all $k \in I_{n+1}$. This is illustrated in figure 6.3 .1 for $n=2$. In general we have that for all $\mu$ the number of $t$-simplices in $B^{n}(v, \mu) \cap A(T)$ is the same for every $T$ with $|T|=t$.

To apply the variable dimension algorithm, in case of integer labelling any point $\mathrm{x}$ receives the standard label of definition 4.2.3, whereas in case of vector labelling each point receives a label as defined in definition 4.8.1 with $b$ equal to $e$. The algorithm is started in $v$ and proceeds in the same way as on $\mathrm{T}^{\mathrm{n}}$ with in the replacement steps the $\mathrm{n}+1$ columns of the matrix defined above in stead of the columns of $T$. A path of adjacent simplices is generated such that the common facet of two adjacent simplices in a region $A(T)$ is T-complete. Using standard arguments we have that the path of simplices either terminates with a completely labelled simplex or goes to infinity. Before we give some convergence theorems we consider the behaviour of the path. We restrict ourselves to vector labelling. 


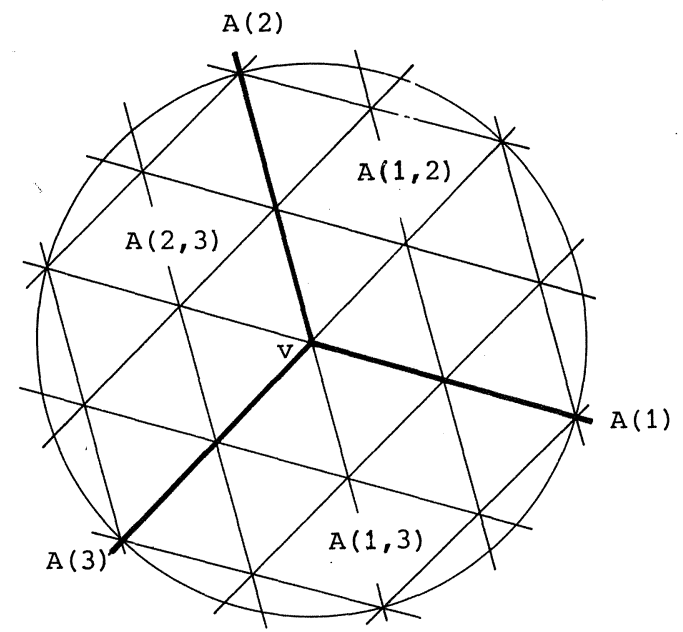

Figure 6.3.1. Illustration of the properties of the $A(n+\sqrt{n+1}) K_{\delta}$ triangulation, $n=2, \mu=2 \delta$.

For some $T$, let $\tau\left(w^{1}, \ldots, w^{t}\right)$ be the common facet of two adjacent simplices generated by the algorithm. Then we have that $\tau$ is $T$-complete and so the system of linear equations

$$
\sum_{i=1}^{t} \lambda_{i} l\left(w^{i}\right)+\sum_{h \notin T} \mu_{h} e(h)=e
$$

has a nonnegative solution $\lambda_{i^{\prime}}^{*} i=1, \ldots, t$ and $\mu_{h^{\prime}}^{*} h \notin T$. First suppose that $n+1 \notin T$. Then it follows from corollary 6.2 .1 that $\sum_{i=1}^{t} \lambda_{i}^{*}<1$. Following the arguments of section 5.6 we have that

(6.3.1) $\quad f_{j}(\bar{x})-\bar{x}_{j}=\alpha \quad j \in T$

and

(6.3.2) $\quad f_{h}(\bar{x})-\bar{x}_{h}<\alpha \quad h \notin T$

where $\alpha=\left(1-\Sigma_{i=1}^{t} \lambda_{i}^{*}\right) / \Sigma_{i=1}^{t} \lambda_{i}^{*}$ and $\bar{x}=\Sigma_{i=1}^{t} \bar{\lambda}_{i} w^{i}$ with $\bar{\lambda}_{i}=\lambda_{i}^{*} / \Sigma_{i=1}^{t} \lambda_{i}^{*}$. Hence if $n+1 \notin T$ the variable dimension restart algorithm traces a path of points $x \in A(T)$ such that for some $\alpha(x)(6.3 .1)$ and (6.3.2) hold. Observe that the number of equations of (6.3.1) is equal to $|T|=t$, whereas $\alpha$ depends on $x$. Suppose now that $n+1 \in T$. Then we have that $\sum_{i=1}^{t} \lambda_{i}^{*}=1$. Hence for $j \in T, j \neq n+1$ we have that 


$$
\sum_{i=1}^{t} \lambda_{i}^{*}\left\{f_{j}\left(w^{i}\right)-w_{j}^{i}\right\}=0
$$

or

(6.3.3) $\quad f_{j}\left(x^{*}\right)-x_{j}^{*}=0 \quad j \in T \backslash\{n+1\}$

with $x^{*}=\sum_{i=1}^{t} \lambda_{i}^{*} w^{i}$. Moreover for $h \notin T$ it follows that

$$
\sum_{i=1}^{t} \lambda_{i}^{*}\left\{f_{h}\left(w^{i}\right)-w_{h}^{i}\right\}+\mu_{h}^{*}=0
$$

or

$$
\text { (6.3.4) } \quad f_{h}\left(x^{*}\right)-x_{h}^{*}<0 \quad h \notin T \text {. }
$$

So a path of points $x \in A(T)$ is traced such that (6.3.3) and (6.3.4) hold. Observe that for each $\mathrm{x}$ the right hand side of (6.3.3) is equal to zero, but that the number of equations in $(6.3 .3)$ is equal to $|T|-1=t-1$.

We give now some theorems concerning the convergence of the algorithm on $\mathrm{R}^{\mathrm{n}}$. First we consider integer labelling. Define $\tilde{B}^{\mathrm{n}}(\mu)$ by

$$
\tilde{B}^{n}(\mu)=\left\{x \in R^{n}\left|\max _{i \in I_{n}}\right| y_{i} \mid=\mu\right\} .
$$

THEOREM 6.3.1. Let $\mathrm{f}$ be a continuous function on $\mathrm{R}^{\mathrm{n}}$ and let $\mathrm{v}$ be the starting point. Assume there exist numbers $\mu>\max _{i}\left|v_{i}\right|$ and $\varepsilon>0$ such that for all $x \in \tilde{B}^{n}(\mu)$ holds

a) $e^{T} x<e^{T} v$ and there is at least one index $j$ such that

$$
f_{j}(x)-x_{j}>\varepsilon
$$

or

b) there exists an index $i \in I_{n}$ such that $x_{i}>v_{i}$, and we have that (i) $f_{j}(x)-x_{j}>f_{i}(x)-x_{i}+\varepsilon$ for at least one index $j \in I_{n^{\prime}} j \neq i$ or (ii) $f_{j}(x)-x_{j}<-\varepsilon$ for all $j \in I_{n}$.

Then the variable dimension restart algorithm applied with integer labelling terminates with a completely labelled simplex for any triangulation $A(\alpha) k_{\delta}$ if $\delta$ is small enough. 
PROOF. Let the grid size be so small that

$$
\text { (6.3.5) } \sup _{x, y \in \sigma} \max _{i \in I_{n}}\left|f_{i}(x)-x_{i}-f_{i}(y)+y_{i}\right|<\frac{1}{2} \varepsilon
$$

for every simplex $\sigma$ such that $\sigma \cap \tilde{B}^{n}(\mu) \neq \varnothing$. For some $T$, let $\sigma\left(w^{1}, \ldots, w^{t+1}\right)$ be a simplex in $A(T)$ such that $\sigma \cap \tilde{B}^{n}(\mu) \neq \not$. Then for any point $x \in \tilde{B}^{n}(\mu)$ no holds at least one of the conditions a) and b). If a) holds we must have that $n+1 \epsilon T$ since $e^{T} x<e^{\top} v$. So if $\sigma$ is generated by the algorithm $\ell\left(w^{i}\right)=n+1$ for at least one index $i=1, \ldots, t+1$. However, there is at least one index $j$ such that $f_{j}(x)-x_{j}>\varepsilon$. So with (6.3.5) it follows that

$$
f_{j}\left(w^{k}\right)-w_{j}^{k}>0 \cdot k=1, \ldots, t+1 \text {, }
$$

implying that $\ell\left(w^{k}\right) \neq n+1$ for all $k$. So a contradiction is obtained. If $b$ ) holds we must have that $i \in T$ since $x_{i}>v_{i}$. If there exists an index $j \neq i$ such that $f_{j}(x)-x_{j}>f_{i}(x)-x_{i}+\varepsilon$ it follows from (6.3.5) that

$$
f_{j}\left(w^{k}\right)-w_{j}^{k}>f_{i}\left(w^{k}\right)-w_{i}^{k} \quad k=1, \ldots, t+1,
$$

implying that $\ell\left(w^{k}\right) \neq i$ for all $k$. If for all $j \in I_{n^{\prime}} f_{j}(x)-x_{j}<-\varepsilon$ then we have that

$$
f_{j}\left(w^{k}\right)-w_{j}^{k}<0 \quad k=1, \ldots, t+1
$$

implying that $\ell\left(w^{k}\right)=n+1$ for all $k$. In both cases $\sigma\left(w^{1}, \ldots, w^{t+1}\right)$ cannot be generated by the algorithm. Therefore the algorithm generates only simplices in the interior of $\operatorname{conv}\left(\tilde{B}^{n}(\mu)\right)$. This proves the theorem.

Theorem 6.3.1 is the analogon of theorem 5.7.1. Theorems analogous to the theorems 5.7.2 and 5.7.3 can easily be given for any element of the subclass A. Here we give only the analogon of theorem 5.7.2. Moreover we restrict ourselves to the $\mathrm{K}_{\delta}$ triangulation.

THEOREM 6.3.2. Let $f$ be a continuous function on $R^{n}$. Assume there exist numbers $\mu>0$ and $\varepsilon>0$ such that for all $x \in \tilde{B}^{\mathrm{n}}(\mu)$ holds that 
a) For some $i, x_{i}=-\mu$ and there exists at least one index $j \in I_{n}$ such that $f_{j}(x)-x_{j}>\varepsilon$.

or

b) For some $i, x_{i}=\mu$ and $f_{j}(x)-x_{j}>f_{i}(x)-x_{i}+\varepsilon$ for at least one index $j \in I_{n^{\prime}} j \neq i$, or $f_{j}(x)-x_{j}<-\varepsilon$ for all $j \in I_{n}$.

Then the variable dimension restart algorithm applied with integer labelling and using the $\mathrm{K}_{\delta}$ triangulation terminates with a completely labelled simplex for any starting point $v$ in the interior of $\operatorname{conv}\left(\tilde{B}^{\mathrm{n}}(\mu)\right)$ if $\delta$ is small enough.

PROOF. For some $T$, let $\sigma\left(w^{1}, \ldots, w^{t+1}\right)$ be a simplex in $A(T)$ and $\sigma \cap \tilde{B}^{n}(\mu) \neq \varnothing$. It can easily be seen that, if the grid size is small enough, there exists an index $i \in T$ such that $\ell\left(w^{k}\right) \neq i, k=1, \ldots, t+1$. Hence only simplices in the interior of $\operatorname{conv}\left(\tilde{B}^{\mathrm{n}}(\mu)\right)$ can be generated.

We conclude this chapter with two convergence theorems on $\mathrm{R}^{\mathrm{n}}$ when vector labelling is used. They are analogous to the theorems 5.7.4 and 5.7.5 concerning the convergence on $\mathrm{T}^{\mathrm{n}}$.

THEOREM 6.3.3. Let $f$ be a continuous function on $\mathrm{R}^{\mathrm{n}}$ and let $\mathrm{v}$ be the starting point. Then the algorithm applied with vector labelling terminates under the same conditions as formulated in theorem 6.3.1.

PROOF. Let the grid size be so small that

$(6.3 .6)$

$$
\sup _{(x, y) \in \sigma} \max _{i \in I_{n+1}}\left|f_{i}(x)-x_{i}-f_{i}(y)+y_{i}\right|<\frac{1}{2} \varepsilon
$$

for every simplex $\sigma$ such that $\sigma \cap \tilde{B}^{n}(\mu) \neq \varnothing$. For some $T$, let $\sigma\left(w^{1}, \ldots, w^{t+1}\right)$ be a simplex in $A(T)$ such that $\sigma \cap \tilde{B}^{n}(\mu) \neq \varnothing$. Let $x$ be a point such that $x \in \tilde{B}^{n}(\mu) n \sigma$. If a) holds we have that $n+1 \in T$. Moreover with (6.3.6) it follows that $f_{j}\left(w^{k}\right)-w_{j}^{k}>0, k=1, \ldots, t+1$. Hence $\bar{f}_{j}(y)-y_{j}>0$ for all $y \in \sigma$, where $\bar{f}$ is the piecewise linear approximation to $f$ with respect to the triangulation. Therefore, by $(6.3 .3)$ or $(6.3 .4)$ cannot be generated by the 
algorithm. If b) holds we have that $i \in T$. If bi) holds it follows that $\bar{f}_{j}(y)-y_{j}>\bar{f}_{i}(y)-y_{i}$ for all $y \in \sigma$ and by (6.3.1) or (6.3.2), $\sigma$ cannot be generated. If bii) holds it follows that for all $y \in \sigma, \bar{f}_{j}(y)-y_{j}<0$ for all $j \in I_{n}$. In particular we have that $\bar{f}_{i}(y)-y_{i}<0$ and by $(6.3 .1)($ if $n+1 \notin T)$ or (6.3.3) (if $n+1 \in T$ ) it follows again that $\sigma$ cannot be generated. Hence only simplices in the interior of $\operatorname{conv}\left(\tilde{B}^{n}(\mu)\right)$ are generated.

As in the previous chapter we have again that in case of an upper-semicontinuous mapping (5.7.5) possibly does not hold for any $\delta>0$. Therefore we need a stronger condition to prove convergence, e.g. in case a) $f_{j}(x)-x_{j}>\varepsilon$ has to be replaced by $f_{j}(z)-z_{j}>0$ for all $z \in B^{n}(x, \delta)$ and $f(z) \epsilon \phi(z)$ for some $0<\delta<\mu-\max _{i}\left|v_{i}\right|$. Then for a triangulation with grid size less than $\delta$ it can easily be seen that if a) holds for some $x \in \tilde{B}^{n}(\mu), \bar{f}_{j}\left(w^{k}\right)-w_{j}^{k}>0$ for all vertices $w^{1}, \ldots, w^{t+1}$ of the simplex $\sigma$ containing $\mathrm{x}$, implying that $\mathrm{n}+1 \notin \mathrm{T}$. The same adaptions have to be made for the condition $b$ ).

Another sufficient condition to prove convergence in case of a mapping is Merrill's condition, as is stated in the next theorem.

THEOREM 6.3.4. Let $\phi$ be an u.s.c. mapping on $R^{n}$. For some $\delta>0$, let there exist $w \in R^{n}$ and $\bar{\mu}>0$ such that for all $x, z \in R^{n}$ with $x \notin B(w, \bar{\mu})$ and $z \in B^{n}(x, \delta)$ holds

$$
(h(x)-x)^{\top}(w-z)>0
$$

for all $\mathrm{h}(\mathrm{x}) \epsilon \phi(\mathrm{x})$. Then the variable dimension restart algorithm applied with vector labelling and using the $A(\alpha) K$ triangulation with $A(\alpha) \epsilon A$ terminates with a completely labelled simplex if the mesh of the triangulation is less than $\delta$.

PROOF. Let $\mu>0$ be such that for all $x \in B^{n}(w, \bar{\mu})$ holds $\max _{i}\left|x_{i}\right|<\mu$. So for all $x$ with $\max _{i}\left|x_{i}\right| \geq \mu$ we have that $x \notin B(w, \bar{\mu})$. Let $v$ be the starting point and define $\mu^{\prime}$ by

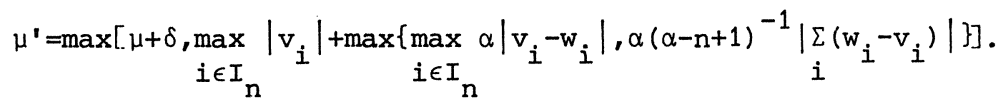


For some $T$, let $\sigma\left(y^{1}, \pi(T)\right)$ be a $t$-simplex in $A(T)$ such that for some $y \in \sigma$, $\max _{i}\left|y_{i}\right|>\mu^{\prime}$. We prove that the system of linear equations

$$
\sum_{i=1}^{t+1} \lambda_{i} \ell\left(w^{i}\right)+\sum_{h \notin T} \mu_{h} e(h)=e
$$

does not have a feasible solution $\lambda_{i}^{*}, i=1, \ldots, t+1$ and $\mu_{h}^{*}, h \notin T$, implying that $\sigma$ cannot be generated by the algorithm. Suppose there exists such a solution. Then we have that

$$
\left[\sum_{i=1}^{t+1} \lambda_{i}^{*} l\left(w^{i}\right)+\sum_{h \notin T} \mu_{h}^{*} e(h)\right]^{\top}\left[(w-y)^{\top}, 0\right]^{\top}=e^{\top}\left[(w-y)^{\top}, 0\right]^{\top}
$$

or by defining $\mu_{n+1}^{*}=0$ if $n+1 \epsilon T$ and letting $f$ be the piecewise linear approximation to $\phi$

$$
\sum_{i=1}^{t+1} \lambda_{i}^{*}\left\{f\left(w^{i}\right)-w^{i}\right\}^{\top}(w-y)+\underset{h \notin T}{\sum} \mu_{h}^{*}\left(w_{h}-y_{h}\right)-\mu_{n+1}^{*} \underset{i=1}{\sum}\left(w_{i}-y_{i}\right)=0
$$

using the fact that $\mu_{n+1}^{*}=1-\Sigma_{i=1}^{t+1} \lambda_{i}^{*}$ (c.f. corollary 6.2.1). Since $\max _{i}\left|y_{i}\right|>\mu^{\prime}$ we have that $\max _{i}\left|w_{i}^{k}\right|>\mu^{\prime}-\delta \geq \mu$. Hence

$$
\left\{f\left(w^{k}\right)-w^{k}\right\}^{\top}(w-y)>0 \quad k=1, \ldots, t+1
$$

Now we prove that $w_{h}-y_{h}>0$ for all $h \notin T$. We first consider the case where, for some $k, y_{k}=\max _{i}\left|y_{i}\right|$. Hence

$$
\mathrm{y}_{\mathrm{k}}>\mu^{\prime}>\mathrm{v}_{\mathrm{k}}+\alpha\left(\mathrm{v}_{\mathrm{h}}-\mathrm{w}_{\mathrm{h}}\right) \quad \mathrm{h} \notin T
$$

Moreover, by the structure of the $A(\alpha) K$ triangulation we have that

$$
\mathrm{y}_{\mathrm{h}} \leq \mathrm{v}_{\mathrm{h}}-\alpha^{-1}\left(\mathrm{y}_{\mathrm{k}}-\mathrm{v}_{\mathrm{k}}\right) \quad \mathrm{h} \notin T
$$

Combining both inequalities we obtain that

$$
\text { (6.3.9a) } \quad y_{h}<v_{h}-\left(v_{h}-w_{h}\right)=w_{h} \quad h \notin T .
$$

In the case where, for some $k,-y_{k}=\max _{i}\left|y_{i}\right|$ we again find by the structure of $A(\alpha)$ 


$$
\mathrm{y}_{\mathrm{h}}=\mathrm{v}_{\mathrm{h}}+\left(\mathrm{y}_{\mathrm{h}}-\mathrm{v}_{\mathrm{h}}\right) \leq \mathrm{v}_{\mathrm{h}}+\left(\mathrm{y}_{\mathrm{k}}-\mathrm{v}_{\mathrm{k}}\right) \quad \mathrm{h} \notin T
$$

Together with $-\mathrm{y}_{\mathrm{k}}>\mu^{\prime} \geq-\mathrm{v}_{\mathrm{k}}+\alpha\left|\mathrm{v}_{\mathrm{h}}-\mathrm{w}_{\mathrm{h}}\right| \geq-\mathrm{v}_{\mathrm{k}}+\mathrm{v}_{\mathrm{h}}-\mathrm{w}_{\mathrm{h}}$ this again implies that (6.3.9b) $\quad y_{h}<w_{h} \quad h \notin T$.

If $\mu_{n+1}^{*}=0$, i.e. $n+1 \in T$, then (6.3.8) and $(6.3 .9 a, b)$ contradict (6.3.7), implying that there is no feasible solution to the system of linear equations. It remains to prove that $\Sigma_{i}\left(w_{i}-y_{i}\right)<0$ if $(n+1) \notin T$. In the case where for some $k, y_{k}=\max _{i}\left|y_{i}\right|$, it follows again by $A(\alpha)$ that

$$
\sum_{i \neq k} y_{i} \geq \sum_{i \neq k} v_{i}-(n-1) \alpha^{-1}\left(y_{k}-v_{k}\right)
$$

Moreover $\mathrm{y}_{\mathrm{k}}>\mathrm{v}_{\mathrm{k}}$ and

$$
\mathrm{y}_{\mathrm{k}}>\mu^{\prime} \geq \mathrm{v}_{\mathrm{k}}+\alpha(\alpha-n+1)^{-1} \sum_{i=1}^{\mathrm{n}}\left(\mathrm{w}_{i}-\mathrm{v}_{i}\right) \text {. }
$$

Hence

$$
\sum_{i=1}^{n}\left(w_{i}-y_{i}\right) \leq \sum_{i=1}^{n}\left(w_{i}-v_{i}\right)+(n-1) \alpha^{-1}\left(y_{k}-v_{k}\right)
$$

and with $(6 \cdot 3.10)$

$$
\sum_{i=1}^{n}\left(w_{i}-y_{i}\right)<0 .
$$

Finally, in the case where for some $k,-y_{k}=\max _{i}\left|y_{i}\right|$ we have that

$$
\begin{aligned}
-\mathrm{y}_{\mathrm{k}}>\mu^{\prime} & \geq-\mathrm{v}_{\mathrm{k}}+\alpha(\alpha-n+1)^{-1}\left|\sum_{i=1}^{n}\left(w_{i}-v_{i}\right)\right| \\
& \geq-v_{k}+(\alpha-n+1)^{-1} \sum_{i=1}^{n}\left(w_{i}-v_{i}\right) .
\end{aligned}
$$

Again from the structure of $A(\alpha)$ it follows that

$$
\sum_{i \neq k}\left(y_{i}-v_{i}\right) \geq(\alpha-n+2)\left(v_{k}-y_{k}\right) .
$$


Hence

$$
\sum_{i=1}^{n}\left(w_{i}-y_{i}\right)<0
$$

So there does not exist a feasible solution to the system of linear equations. Hence only simplices can be generated in the bounded set $\operatorname{conv}\left(\tilde{B} n\left(\mu^{\prime}\right)\right)$.

Observe that in the proof of the theorem $\mu^{\prime} \geq \max \alpha\left|v_{i}-w_{i}\right|$. Hence, if $\alpha \rightarrow \infty$,

$$
i \in I_{n+1}
$$

$\mu^{\prime}$ is unbounded unless $v=w$. Therefore the theorem is not true if the $\mathrm{K}$ triangulation is used, except in the case where $v=w$.

Finally, we note that the condition "for some $\delta>0$ " can be changed into the strong condition of Merrill "for all $\delta>0$ ". In this case the algorithm converges for every grid size.

\subsection{RECENT DEVELOPMENTS.}

As on $S^{\mathrm{n}}$ the variable dimension restart algorithm can be interpreted with $n+1$ points on an extra level (c.f. Todd [1978b], Barany [1979a], Van der Laan and Talman [1979c,1980c]). Therefore, let $R^{n} \times\{1\}$ be the natural level and $R^{n} \times\{0\}$ the artificial level. Now we choose $n+1$ points $u(i) \in R^{n}$ such that

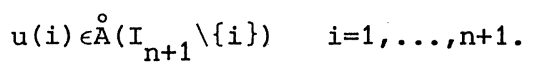

Let the n-simplex $\sigma$ be the convex hull of the points $u(i)$ and let $G$ be the collection of $(n+1)$-simplices obtained by connecting each grid point $x \in \AA(T)$ on level one with the vertices $u(i), i \notin T$, on level zero. Then we have that $G$ triangulates the set $(\sigma \times\{0\}) \cup\left(R^{n} \times(0,1]\right)$. The proof of this proposition can be found in Todd [1978b]. Observe that this triangulation is not locally finite, but still satisfies the property of theorem 2.3.5. We present now briefly the research of Todd [1978b] about the variable dimension restart algorithm of Van der Laan and Talman [1979a,b] . Let $f$ be a continuous function on $R^{n}$ and let the points $u(i)$ be defined by 


$$
\begin{aligned}
& u(i)=\delta(e-(n+1) e(i)+w) \quad i=1, \ldots, n \\
& u(n+1)=\delta(e+w),
\end{aligned}
$$

where $\delta$ is the grid size of the $k$ (or $J_{1}$ ) triangulation of $R^{n} \times\{1\}$ and $w$ is the $n$-dimensional vector such that $w_{j}=(2 n-2 j+1) / 2 n, j=1, \ldots, n$. Using vector labelling the system of linear equations is initiated with $B \mu=b$ where $b=(0, \ldots, 0,1)^{\top}$ and $B$ is the matrix with $b(j)=\left((\delta w-u(j))^{\top}, 1\right)^{\top}$. Todd proves that the algorithm traces a path of zeroes of the piecewise linear approximation to the function

$$
h(x, t)=(1-t)(\delta w-x)+t(f(x)-x)
$$

Using this fact it is proved that Merrill's condition is sufficient to guarantee that the algorithm converges. So the condition is sufficient with $K$ the underlying triangulation. However, observe that the system of linear equations is not initiated with $I \mu=e$ as in the previous section. Starting with $I \mu=e$ has the advantage that when the dimension of the simplex is small, the basis matrix is sparse, which yields that efficient linear programming steps can be made.

Todd gives also a technique to accelerate the algorithm. Therefore he uses the set-up of the accelerated algorithms in Saigal [1977] and Saigal and Todd [1978]. He proves that in the limit an approximation in a certain stage can be obtained in $\mathrm{n}+1$ iterations and that the algorithm converges o-quadratically, when the function is continuously differentiable with a Lipschitz continuous derivative.

A note about the path of the variable dimension restart algorithm was written by Kojima [1980]. He showed that the same path can be obtained by utilizing the differential equations approach when the function is continuously differentiable.

In Van der Laan and Talman [1980b] the triangulation of $s^{n} \times[0,1]$ as given in section 5.5 is used to construct a triangulation of $S^{n} \times[1, \infty)$ which allows for any factor of grid refinement between two successive levels. We treat this matter in chapter 8.

The variable dimension restart algorithm is generalized in Van der Laan and Talman $[1978 \mathrm{a}, \mathrm{b}]$. In the first paper an algorithm is given which can be applied to compute a fixed point of a function on the product space of unit simplices. The second paper is closely related and presents a class of 
algorithms on $R^{n}$. One of the extreme cases of this class is the algorithm presented in this chapter. The other extreme case is an algorithm with $2 \mathrm{n}$ labels when integer labelling is used and a system of $2 \mathrm{n}$ linear equations when vector labelling is used. In addition, starting with a point $v$, a search in $2 n$ directions can be made. The class of algorithms is treated in full detail by Talman [1980]. An interpretation of the "2n-case" with points on an extra level can be found in Todd and Wright [1979] and Todd [1980]. Interpretations with extra points for any element of the class are given by Van der Laan and Talman [1979c] (see also Talman [1980]). In Todd and Wright [1979] i.t is showed that the 2 -case is very appropriate to be used for antipodal fixed point algorithms. Finally we mention a paper of wright [1979]. He generalized the variable dimension restart algorithm to an algorithm with $2^{n}$ directions. As argued by wright the algorithm can be considered to be dual to the $2 n$-case.

\subsection{COMPUTATIONAL RESULTS.}

To test the algorithm the three pure exchange economy problems of Scarf [1967b] were again solved. The problems were converted to problems in $\mathrm{R}_{+}^{\mathrm{n}}$ by setting the price of one of the commodities equal to one. The algorithm was applied with vector labelling and the $\mathrm{K}$ triangulation. A point $x$ outside int $R_{+}^{n}$ received the label $e(i)$ with $i$ the smallest index with $x_{i} \leq 0$, whereas a point $x \in$ int $R_{+}^{n}$ received the same label as in section 5.8 . In the first stage the starting point was the point $x$ with $x_{i}=1$, $i=1, \ldots, n$ and the mesh was $(n+1)(n+2)^{-1} \sqrt{n}$. The approximate fixed point $w^{*}(m)$ on stage $m$ was chosen to be the starting point at stage $m+1, m=1,2, \ldots$. The grid size $\delta_{m+1}$ at stage $\mathrm{m}+1$ is defined by

$$
\delta_{m+1}=\frac{1}{2} \delta_{m} \text { if } h_{m} \geq \frac{1}{2} \delta_{m} \text { and } \delta_{m+1}=h_{m} \text { if } h_{m}<\frac{1}{2} \delta_{m}
$$

with $h_{m}$ as defined in section 5.8. The results are given in table 6.5.1. 
Table 6.5.1. Three pure exchange economy models, $n=4,7$ and $9, M=$ mesh $G_{m} / \sqrt{ }$, $N$ is the total number of iterations, $E=\max \left|\left(g_{i}-a_{i}\right) / a_{i}\right|$. $i \in I_{n+1}$

\begin{tabular}{c|c|c|c|c} 
Dimension & $\mathrm{m}$ & $\mathrm{M}$ & $\mathrm{N}$ & $\mathrm{E}$ \\
\hline 4 & 5 & $\begin{array}{r}6.10^{-3} \\
10^{-5}\end{array}$ & $\begin{array}{l}47 \\
69\end{array}$ & $\begin{array}{l}210^{-5} \\
<10^{-12}\end{array}$ \\
\hline 7 & 7 & $5.10^{-3}$ & 72 & $610^{-6}$ \\
& 5 & $910^{-5}$ & 80 & $10^{-9}$ \\
\hline 9 & 6 & $310^{-3}$ & 102 & $10^{-6}$ \\
& 5 & $10^{-4}$ & 123 & $210^{-10}$ \\
\hline
\end{tabular}

In the three problems above the number of function evaluations is equal to the number of linear programing steps. The algorithm was also applied on the first production economy problem of Scarf [1973].

To achieve an accuracy of less than $5.10^{-3}$ the algorithm needed 176 function evaluations and 184 linear programming steps, i.e. 8 times step 4 was performed, bringing a unit vector in the system of linear equations. A comparison of the number of iterations with the results as reported by Todd [1978a] shows that the variable dimension restart algorithm takes significantly fewer iterations than the algorithm of Merrill and the method of Eaves and Saigal. Even in the case that quasi-Newton steps were used our results are better or comparable. of course, taking quasi-Newton steps can also be implemented in our algorithm.

The algorithm was also applied on the problems 1 and 2 of Saigal [1977c]. Problem 1 is a 4-dimensional unconstrained optimization problem, originally due to Colville [1968]. Problem 2 is a 20-dimensional version of a problem considered by Kellogg, $\mathrm{Li}$ and Yorke [1976]. For both problems the grid size in stage 1 is set equal to 2 , whereas $\delta_{m+1}=\frac{1}{2} \delta_{m}$ for all $m \geq 1$. The starting point was $(-3,-1,-3,-1)^{\top}$ for problem 1 , and the zero point for problem 2 . The results are given in table 6.5.2. 
Table 6.5.2. Problem 1, unconstrained optimization, $E=\max _{i \in I_{n}}\left|\frac{\partial f(x)}{\partial x_{i}}\right|_{x=w}{ }^{*}(m) ;$

Problem 2, 20-dimensional fixed point problem,

$\left.E=\max _{i \in I_{n}} \mid f_{i} w^{*}(m)\right)-w_{i}^{*}(m) \mid$,

$\mathrm{N}$ is the total number of iterations.

\begin{tabular}{c|cc|c} 
Problem & $\mathrm{m}$ & $\mathrm{N}$ & $\mathrm{E}$ \\
\hline 1 & 5 & 51 & 2.6 \\
& 7 & 89 & .037 \\
& 9 & 107 & $210^{-4}$ \\
\hline 2 & 1 & 37 & .38 \\
& 3 & 90 & $10^{-3}$ \\
& 5 & 136 & $10^{-7}$ \\
\hline
\end{tabular}

Compared with the results of Saigal [1977c] it follows that the variable dimension algorithm again takes much fewer iterations than the homotopy algorithm. Especially, the number of iterations is very small in the first stage. Finally we remark that for both problems the $\mathrm{K}$ triangulation was used. For the impact of the $\mathrm{A}\left(\alpha^{*}\right) \mathrm{K}$ triangulation we refer the reader to Talman [1980]. 


\subsection{INTRODUCTION.}

In this chapter we consider the concept of the orientation of simplices. Each completely labelled simplex receives an index being an element of the set $\{+1,-1\}$. It is discussed by several authors that this index is closely related to the Brouwer degree of a mapping (c.f. Charnes, Garcia and Lemke [1977], Prüfer [1977], Garcia and Zangwill [1978], Peitgen and Prüfer [1979] and Prüfer and Siegberg [1979]). The history of the Brouwer degree can be found in siegberg [1980]. An orientation or index theory for bimatrix games is introduced by Shapley [1974] and is generalized by Lemke and Grotzinger [1976]. Deeper and more abstract discussions can be found in Eaves and Scarf [1976] and Todd [1976c]. In this chapter we discuss the relationship between oriented simplices and fixed point algorithms. We restrict ourselves to integer labelling. The generalization to vector labelling can easily be made. In section 2 we give some definitions and lemmas and we prove the wellknown result that the sum of the indices of the completely labelled simplices in $S^{n}$ is equal to one if the labelling rule satisfies the conditions of Sperner's lemma. We will also simplify the definition of the orientation of a completely labelled simplex. In section 3 this simplification is used to define the orientation of a $(T \cup\{j\})$-complete simplex of a region $A(T)$. We give a lower bound on the number of comnletely labelled simplices by considering all ( $T \cup\{j\})$-complete simplices in $A(T)$ with $|T|=n-1$. In section 4 we prove that the variable dimension algorithm always generates a positive oriented completely labelled simplex. Conditions for the existence of at least $\mathrm{k}$ completely labelled simplices for some odd number $k$ are given. Using the concept of orientation it is shown that under these conditions the variable dimension restart algorithm can be used to generate at least $\mathrm{k}$ completely labelled simplices. 


\subsection{ORIENTATION.}

Let $\mathrm{S}^{\mathrm{n}}$ be triangulated in the standard way with grid size $\mathrm{m}^{-1}$ and let $\sigma=$ $\sigma\left(y^{1}, \pi\left(I_{n}\right)\right)$ be a completely labelled simplex. Then we define the orientation of $\sigma$, to be denoted by or $\sigma$, as follows.

DEFINITION 7.2.1. The orientation of a completely labelled simplex $\sigma\left(y^{1}, \pi\left(I_{n}\right)\right)$ is defined by

$$
\text { or } \sigma=\operatorname{sign} \operatorname{det}\left[y^{j_{1}} y^{j_{2}} \cdot \ldots y^{j_{n+1}}\right]
$$

where $j_{i}$ is the index such that $\ell\left(y^{j}\right)=i, i=1, \ldots, n+1$.

We also define the orientation of an $I_{n}$-complete $(n-1)$-facet $\tau$ of a simplex $\sigma\left(y^{1}, \pi\left(I_{n}\right)\right)$ where either $\sigma$ is completely labelled or $\sigma$ has two $I_{n}$-complete facets.

DEFINITION 7.2.2. Let $\tau\left(y^{j_{1}}, \ldots, y^{j}{ }^{j}\right)$ be an $I_{n}$-complete facet of a simplex $\sigma\left(y^{1}, \pi\left(I_{n}\right)\right)$ and let the vertices be ordered in such a way that $y^{j_{i}}$ has label $i, \quad i=1, \ldots, n$. Let $y^{j_{n+1}}$ be the vertex of $\sigma$ oppposite to $\tau$. Then the orientation of $\tau$ with respect to $\sigma$, to be denoted by or $\sigma \tau$, is defined by

$$
\operatorname{or}_{\sigma} \tau=\operatorname{sign} \operatorname{det}\left[y^{j_{1}} y^{j_{2}} \cdot \ldots y^{j_{n}} y^{j_{n+1}}\right]
$$

COROLLARY 7.2.3. Let $\sigma\left(\mathrm{y}^{1}, \pi\left(I_{\mathrm{n}}\right)\right)$ be a completely labelled simplex and $\tau$ be the $I_{n}$-complete facet of $\sigma$. Then

$$
\text { or } \sigma=\text { or }_{\sigma} \tau \text {. }
$$

Both the definitions 7.2.1 and 7.2.2 hold for any triangulation of $\mathrm{s}^{\mathrm{n}}$. Utilizing the structure of the standard triangulation of $\mathrm{S}^{\mathrm{n}}$ we rewrite the definitions in another notation. It will appear that using this notation the definitions can easily be generalized to ( $T$ \{ $j\})$-complete t-simplices and T-complete (t-1)-simplices in $A(T), T \subset I_{n+1}$ and $|T| \leq n$. Recall that $q(j)$ is the $j$-th column of the $(n+1) \times(n+1)$ triangulation matrix $Q$ as defined in chapter 5 . Then for each vertex $w$ of the given triangulation of $s^{n}$ there exist nonnegative integers $\alpha_{1}(w), \ldots, \alpha_{n}(w)$ 
such that

$$
w=e(1)+\sum_{j=1}^{n} \delta \alpha_{j}(w) q(j)
$$

with $\delta=\mathrm{m}^{-1}$.

In the following, for a simplex $\sigma\left(y^{1}, \pi\left(I_{n}\right)\right)$ of the triangulation, let $\alpha^{i}$ be the n-dimensional vector $\left(\alpha_{1}^{i}, \ldots, \alpha_{n}^{i}\right)^{r}, i=1, \ldots, n+1$, with $\alpha_{j}^{i}=\alpha_{j}\left(y^{i}\right)$, $j=1, \ldots, n$.

LEMMA 7.2.4. For any simplex $\sigma\left(y^{1}, \pi\left(I_{n}\right)\right)$ holds

$$
\operatorname{det}\left[y^{1} \cdot \cdot y^{n+1}\right]=\delta^{n} \operatorname{det}\left[\begin{array}{c}
1 \cdot \cdot \alpha^{1} \\
\alpha^{1} \cdot \alpha^{n+1}
\end{array}\right] \text {. }
$$

PROOF. Since $y^{i}=e(1)+\sum_{j=1}^{n} \delta \alpha_{j}^{i} q(j)$ we have that $y^{i}=\left(1-\delta \alpha_{1}^{i}, \delta \alpha_{1}^{i}-\delta \alpha_{2}^{i}, \ldots\right.$ $\left.\ldots, \delta \alpha_{n-1}^{i}-\delta \alpha_{n}^{i}, \delta \alpha_{n}^{i}\right)^{\top}$. So adding successively the $i-$ th row to the $(i-1)-$ th row, $i=n+1, n, \ldots, 2$, we obtain

$$
\operatorname{det}\left[y^{1} \cdot \cdot y^{n+1}\right]=\operatorname{det}\left[\begin{array}{ccc}
1 \cdot \cdots \alpha^{1} \\
\delta \alpha^{1} \cdot \cdot \delta \alpha^{n+1}
\end{array}\right]=\delta^{n} \operatorname{det}\left[\begin{array}{c}
1 \cdot \cdot 1 \\
\alpha^{1} \cdot \alpha^{n+1}
\end{array}\right] .
$$

LEMMA 7.2.5. Let $\sigma\left(\mathrm{y}^{1}, \pi\left(I_{n}\right)\right)$ be a simplex of the triangulation of $S^{n}$. Then

$$
\operatorname{det}\left[\begin{array}{c}
1 \cdots \cdot 1 \\
\alpha^{1} \cdots \alpha^{n+1}
\end{array}\right]= \pm 1
$$

PROOF. Since $\sigma=\sigma\left(y^{1}, \pi\left(I_{n}\right)\right)$ we have that $y^{i+1}=y^{i}+\delta q\left(\pi_{i}\right), i=1, \ldots, n$. Hence $\alpha^{i+1}-\alpha^{i}=e\left(\pi_{i}\right), i=1, \ldots, n$ and therefore

$$
\begin{aligned}
\operatorname{det}\left[\begin{array}{ccc}
1 \cdots & 1 \\
\alpha^{1} \cdots \alpha^{n+1}
\end{array}\right] & =\operatorname{det}\left[\begin{array}{cccc}
1 & 0 & \cdots & 0 \\
\alpha^{1} \alpha^{2}-\alpha^{1} & \ldots & \alpha^{n+1}-\alpha^{n}
\end{array}\right]= \\
& =\operatorname{det}\left[\begin{array}{cccc}
1 & 0 & \cdots & 0 \\
\alpha^{1} e\left(\pi_{1}\right) \ldots & e\left(\pi_{n}\right)
\end{array}\right]= \pm 1
\end{aligned}
$$

The lemmas 7.2.4 and 7.2.5 allow for the alternative definitions of or $\sigma$ and or ${ }_{\sigma}{ }$, given in the next corollary.

COROLLARY 7.2.6. a) Let $\sigma\left(y^{1}, \pi\left(I_{n}\right)\right)$ be a completely labelled simplex in $s^{n}$ and let $j_{i}$ be the index such that $\ell\left(y^{j}{ }^{j}\right)=i, i=1, \ldots, n+1$. Then 


$$
\operatorname{or} \sigma=\operatorname{det}\left[\begin{array}{ccc}
1 & \cdots & 1 \\
j_{1} & \ldots & j_{n+1}^{j_{n}}
\end{array}\right] \text {. }
$$

b) Let $\tau\left(y^{j_{1}}, \ldots, y^{j}{ }^{j}\right)$ be an $I_{n}$-complete facet of a simplex $\sigma\left(y^{1}, \pi\left(I_{n}\right)\right)$ such that $\ell\left(y^{j_{i}}\right)=i, i=1, \ldots, n$ and let $y^{j_{n+1}}$ be the vertex of $\sigma$ opposite to $\tau$. Then

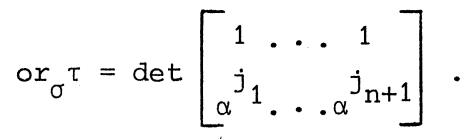

In the next sections we combine the orientation theory with the variable dimension restart algorithm treated in the chapters 5 and 6 . Here we use the variable dimension algorithm of Kuhn, discussed in chapter 4, to prove that the sum of the orientations of the completely labelled simplices in $\mathrm{S}^{\mathrm{n}}$ is equal to one if the labelling rule is proper. Remember that Kuhn's algorithm is a special case of the algorithm presented in chapter 5 . Analogously it will appear that the definition of the orientation of lowerdimensional simplices to be given below is a special case of a more general definition to be given in section 3 .

Consider the sets $s^{n}(k)$ as defined in definition 4.4.1.c, i.e. $s^{n}(k)=$ $\left\{x \in s^{n} \mid x_{i}=0, i=k+1, \ldots, n+1\right\}, k=1, \ldots, n+1$. Recall that $s^{n}(n+1)=s^{n}$ and $s^{n}(n)=s_{n+1}^{n}$. Let $w$ be a vertex of the triangulation of $s^{n}$ in $s^{n}(k)$. Since $w_{i}=0, i=k+1, \ldots, n+1$,it follows that there exist nonnegative integers $\alpha_{j}$, $j=1, \ldots, k-1$, such that

$$
w=e(1)+\sum_{j=1}^{k-1} \delta \alpha_{j} q(j) .
$$

In the following, for a $(k-1)$-simplex $\sigma\left(y^{1}, \pi\left(I_{k-1}\right)\right)$ of the triangulation of $\mathrm{s}^{\mathrm{n}}(\mathrm{k})$, let $\alpha^{\mathrm{i}}$ be the $(\mathrm{k}-1)$-dimensional vector of nonnegative integers $\alpha_{1}^{i}, \ldots, \alpha_{k-1}^{i}$ corresponding to $y^{i}, i=1, \ldots, k$.

DEFINITION 7.2.7. Let $\sigma\left(y^{1}, \pi\left(I_{k-1}\right)\right)$ be an $I_{k}$-complete $(k-1)$-simplex in $s^{n}(k)$ and let $j_{i}$ be the index such that $l\left(y^{j}\right)=i, i=1, \ldots, k$. Then

$$
\operatorname{or} \sigma=\operatorname{det}\left[\begin{array}{ccc}
1 & \ldots & 1 \\
\alpha^{j_{1}} & \ldots & \alpha^{j_{k}}
\end{array}\right] \text {. }
$$


DEFINITION 7.2 .8 . Let $\tau\left(\mathrm{y}^{j_{1}}, \ldots, \mathrm{y}^{j_{k-1}}\right)$ be an $I_{k-1}$-complete facet of a $(k-1)$-simplex $\sigma\left(y^{1}, \pi\left(I_{k-1}\right)\right)$ in $S^{n}(k)$ and let $j_{i}$ be the index such that $\ell\left(y^{j}\right)=i, i=1, \ldots, k-1$. Let $y^{j_{k}}$ be the vertex of $\sigma$ onposite to $\tau$. Then

$$
\operatorname{or}_{\sigma} \tau=\operatorname{det}\left[\begin{array}{ccc}
1 & \ldots & 1 \\
\alpha^{j_{1}} & \ldots & \alpha_{k}^{j_{k}}
\end{array}\right] \text {. }
$$

of course, it follows again that, if $\sigma$ is $I_{k}$-complete and $\tau$ is the $I_{k-1}$ complete facet of $\sigma$, or $\sigma=$ or $_{\sigma} \tau$. Moreover we have that the determinant is equal to \pm 1 .

We prove now some lemmas (see also Allgower and Georg [1980]).

LEMMA 7.2.9. Let $\sigma\left(\mathrm{y}^{1}, \pi\left(\mathrm{I}_{\mathrm{k}-1}\right)\right)$ be a $(\mathrm{k}-1)$-simplex in $\mathrm{s}^{\mathrm{n}}(\mathrm{k})$ having two $I_{k-1}$-complete facets $\tau^{1}$ and $\tau^{2}$. Then

$$
\operatorname{or}_{\sigma} \tau^{1}=\text {-or }_{\sigma} \tau^{2}
$$

PROOF. Let $y^{t_{1}}$ and $y^{t_{2}}$ be the two vertices having the same label and let

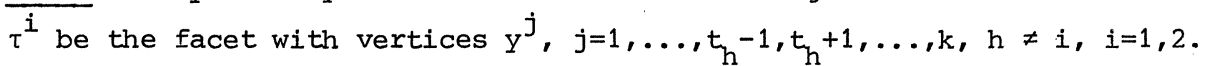
Let $j_{i}, i=1, \ldots, k$ be the permutation of the elements of $I_{k}$ such that $y_{k}^{j_{k}}=y^{t_{2}}$ and $l\left(y^{j_{i}}\right)=i, i=1, \ldots, k-1$. Let $s \in I_{k-1}$ be the index such that $y^{t_{1}=y^{j}}{ }^{y}$. Then

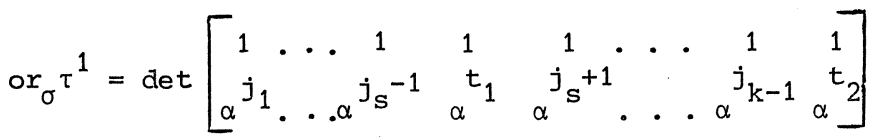

and

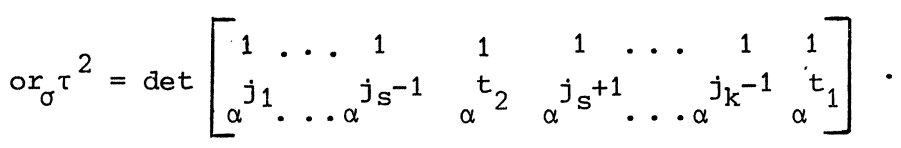

Clearly or $\sigma^{1}=$-or $_{\sigma} \tau^{2}$

LEMMA 7.2.10. Let $\tau$ be an $I_{k-1}$-complete common facet of two $(k-1)-$ simplices $\sigma_{1}\left(y^{1}, \pi\left(I_{k-1}\right)\right)$ and $\sigma_{2}\left(\bar{y}^{-1}, \bar{\pi}\left(I_{k-1}\right)\right)$ in $s^{n}(k)$. Then

$$
\operatorname{or}_{\sigma_{1}} \tau=-\operatorname{or}_{\sigma_{2}} \tau \text {. }
$$


PROOF. Let $\tau$ be the facet of $\sigma_{1}$ with vertices $y^{1}, \ldots, y^{i-1}, y^{i+1}, \ldots, y^{k}$. Then $\sigma_{2}$ is obtained from $\sigma_{1}$ by replacing $y^{i}$. It can easily be verified that the vertex of $\sigma_{2}$ opposite to $\tau$ is equal to $\bar{y}^{-i}=y^{i-1}+y^{i+1}-y^{i}$ where $i-1=k$ if $i=1$ and $i+1=1$ if $i=k$. Let $j_{1}, \ldots, j_{k}$ be the permutation of the elements of $I_{k}$ such that $y^{j_{k}}=y^{i}$ and $l(y)=h, h \in I_{k-1}$. Then

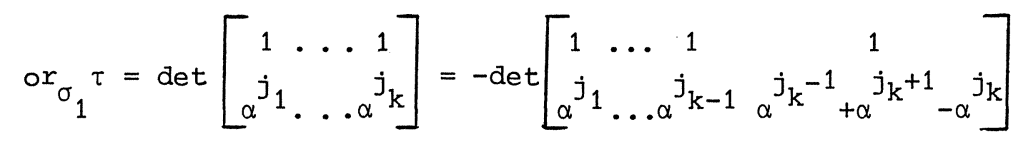

$$
\begin{aligned}
& =-\operatorname{det}\left[\begin{array}{cccc}
1 & \cdot & 1 & 1 \\
\alpha^{j} \cdot & \cdot & \alpha^{j_{k-1}} & \bar{\alpha}
\end{array}\right]=- \text { or } \sigma_{2} \tau
\end{aligned}
$$

where $\bar{\alpha}=\left(\bar{\alpha}_{1}, \ldots, \bar{\alpha}_{k-1}\right)^{\top}$ is such that $\bar{y}^{i}=e(1)+\sum_{j=1}^{k-1} \delta \bar{\alpha}_{j} q(j)$.

LEMMA 7.2.11. For $k \leq n$, let $\tau\left(y^{1}, \pi\left(I_{k-1}\right)\right)$ be an $I_{k}$-complete simplex in $S^{n}(k)$. Let $\sigma\left(y^{1}, \pi\left(I_{k}\right)\right)$ be the k-simplex of $S^{n}(k+1)$ having $\tau$ as a facet. Then

$$
\text { or } \tau=\text { or }_{\sigma} \tau \text {. }
$$

PROOF. In $s^{n}(k)$ we have that $y^{i}=e(1)+\sum_{j=1}^{k-1} \delta \alpha_{j}^{i} q(j), i=1, \ldots, k$. In $\mathrm{s}^{\mathrm{n}}(\mathrm{k}+1)$ we have that $\mathrm{y}^{i}=e(1)+\sum_{j=1}^{k} \delta \alpha_{j}^{-i} q(j), i=1, \ldots, k$, with

$$
\begin{array}{ll}
\alpha_{j}^{i}=\alpha_{j}^{i} & j=1, \ldots, k-1 \\
\alpha_{j}^{i}=0 & j=k .
\end{array}
$$

Moreover, the vertex of $\sigma$ opposite to $\tau$ is equal to $y^{k+1}=e(1)+\Sigma_{j=1}^{k} \delta \alpha_{j}^{-k+1} q(j)$ with $\bar{\alpha}_{j}^{k+1}=\alpha_{j}^{k}, j=1, \ldots, k-1$ and $\alpha_{j}^{k+1}=1$. Let $j_{i}$ be the index such that $\ell\left(y^{j}\right)=i, i=1, \ldots, k$ and $y^{j+1}=y^{k+1}$.

Hence

$$
\begin{aligned}
& \text { or } \tau=\operatorname{det}\left[\begin{array}{ccc}
1 & \ldots & 1 \\
\alpha_{1} & \ldots & \alpha_{k}
\end{array}\right]=\operatorname{det}\left[\begin{array}{cccc}
1 & . & 1 & 1 \\
\alpha^{j_{1}} & \cdots & \alpha^{j_{k}} & \alpha^{k} \\
0 & \cdots & 0 & 1
\end{array}\right]= \\
& =\operatorname{det}\left[\begin{array}{cccc}
1 & \ldots & 1 & 1 \\
\bar{\alpha}^{j_{1}} & \ldots & \bar{\alpha}^{j_{k}} & \bar{\alpha}^{j_{k+1}}
\end{array}\right]=\text { or }_{\sigma} \tau \text {. }
\end{aligned}
$$


The following theorem states that the sum of the orientations of the $I_{k}-$ complete simplices in $s^{n}(k)$ is equal to 1 .

THEOREM 7.2.12. Let $O(k)$ be the set of $I_{k}$-complete simplices in $S^{n}(k)$ and let the labelling rule be such that the conditions of Sperner's lemma are satisfied. Then

$$
\sum_{\sigma \in \mathrm{O}(\mathrm{k})} \text { or } \sigma=1 \quad \mathrm{k}=1, \ldots, \mathrm{n}+1
$$

PROOF. We prove this theorem by induction on $k$. Clearly, for $k=1$ we have that $e(1)$ is the only $I_{1}$-complete zero-simplex of $s^{n}(1)$ and $\operatorname{or}\{e(1)\}=\operatorname{det} 1=1$. Let the theorem be true for $k=j$. By applying the steps of Kuhn's variable dimension algorithm, paths of j-simplices in $s^{n}(j+1)$ will be generated such that the common facets of two adjacent simplices are $I_{j}$-complete. Three different types of paths can occur.

a) Starting with a $j$-simplex in $S^{n}(j+1)$ having an $I_{j}$-complete $(j-1)-$ simplex $\tau$ in $S^{n}(j)$ as a facet, the algorithm generates an $I_{j+1}$-complete simplex $\sigma$ in $S^{\mathrm{n}}(j+1)$, or conversely. By applying the lemmas 7.2.9, 7.2.10, 7.2.11 and corollary 7.2.3 we have that or $\tau=$ or $\sigma$.

b) Starting with a $j$-simplex in $S^{n}(j+1)$ having an $I_{j}$-complete $(j-1)-$ simplex $\tau_{1}$ in $s^{n}(j)$ as a facet, the algorithm generates a $j$-simplex in $S^{n}(j+1)$ having an $I_{j}$-complete $(j-1)$-simplex $\tau_{2}$ in $S^{n}(j)$ as a facet. From the lemmas 7.2.9, 7.2.10 and 7.2.11 it follows that or $\tau_{1}=$-or $\tau_{2}$.

c) Starting with an $I_{j+1}$-complete $j$-simplex $\sigma_{1}$ in $s^{n}(j+1)$ an $I_{j+1}$-complete $j$-simplex $\sigma_{2}$ in $S^{n}(j+1)$ is generated. From corollary 7.2 .3 and the lemmas 7.2 .9 and 7.2 .10 it follows that or $\sigma_{1}=-$ or $\sigma_{2}$.

By the same arguments as in section 4.4 we have that two different paths are disjunct. So, combining these three cases we conclude $\Sigma_{\sigma \in O(j+1)}$ or $\sigma=$ $\Sigma_{\tau \in O(j)}$ or $\tau$, which proves that $\Sigma_{\sigma \in O(k)}$ or $\sigma=1, k=1, \ldots, n+1$

The proof of the theorem is illustrated in figure 7.2.1. The path of $\tau_{1}$ to $\sigma_{1}$ is of type 1 , or $\sigma_{1}=$ or $\tau_{1}=1$; the path between $\tau_{2}$ and $\tau_{3}$ is of type 2 , 
or $\tau_{2}=$-or $\tau_{3}=-1$ i and the paths between $\sigma_{2}$ and $\sigma_{3}$ respectively $\sigma_{4}$ and $\sigma_{5}$ are of type 3 with or $\sigma_{2}=-o r \sigma_{3}=-1$ and or $\sigma_{4}=-$ or $\sigma_{5}=-1$. Observe that it is impossible to generate a path between two simplices with the same orientation.

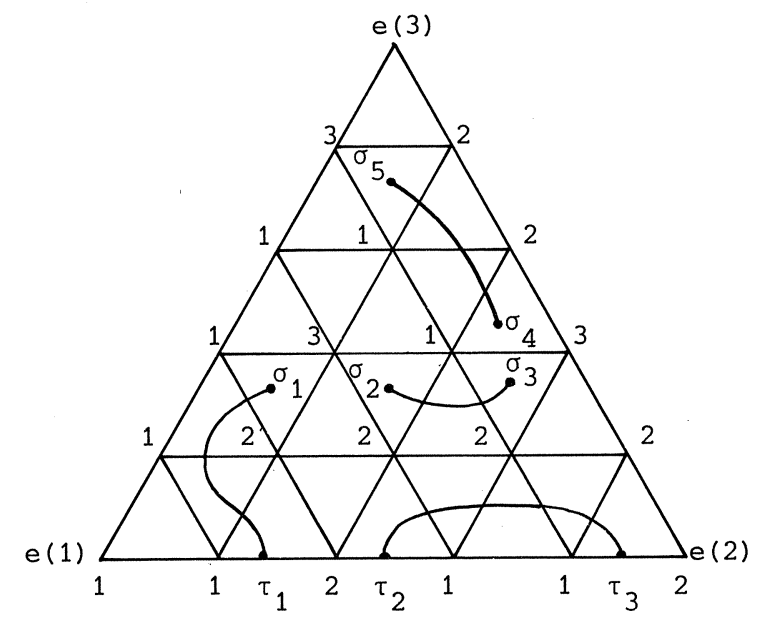

Figure 7.2.1. Illustration of theorem 7.2.12; $\operatorname{or}\{e(1)\}=1$;

$$
\begin{aligned}
& \text { or } \tau_{1}=\text { or } \tau_{3}=1 \text {, or } \tau_{2}=-1 ; \text { or } \sigma_{1}=\text { or } \sigma_{3}=\text { or } \sigma_{5}=1 \text {, } \\
& \text { or } \sigma_{2}=\text { or } \sigma_{4}=-1 \text {. }
\end{aligned}
$$

In particular theorem 7.2.12 holds if $k=n$ and $k=n+1$. Since we have a proper labelling there are no $I_{n}$-complete simplices in $s_{i}^{n}$, $i \neq n+1$. So we have that the sum of the orientations of the completely labelled simplices of $S^{n}$ is equal to the sum of the orientations of the $I_{n}$-complete simplices in bd $\mathrm{s}^{\mathrm{n}}$. A more general result can be found in Prüfer and Siegberg [1979].

\subsection{THE VARIABLE DIMENSION RESTART ALGORITHM.}

In this section we apply the concept of orientation to the variable dimension algorithm treated in chapter 5 . Let the arbitrarily chosen grid point $\mathrm{v}$ be the starting point of the variable dimension algorithm. Recall that for any $T \in I_{n+1},|T| \leq n$, the regions $A(T)$ are defined by 
$A(T)=\left\{x \in S^{n} \mid x=v+\sum_{j \in T} \lambda_{j} q^{(j)}\right.$ for nonnegative numbers $\left.\lambda_{j}, j \in T\right\}$.

For any grid point $w \in A(T),|T|=t$, there is a unique $t$-dimensional vector $\alpha$ of nonnegative integers $\alpha_{j}, j \in T$, such that

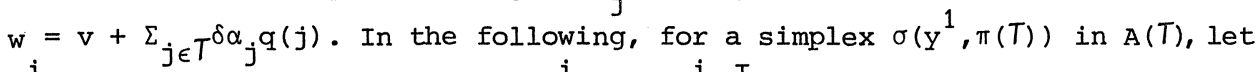
$\alpha^{i}$ be the t-dimensional vector $\left(\alpha_{s_{1}}^{i}, \ldots, \alpha_{s_{t}}^{i}\right)^{\top}$ such that

$$
y^{i}=v+\sum_{s_{j} \in T} \delta \alpha_{s_{j}^{i}} q\left(s_{j}\right),
$$

where $\left(s_{1}, \ldots, s_{t}\right)$ is the permutation of the elements of $T$ such that $s_{i}<s_{i+1}, i=1, \ldots, t-1$.

DEFINITION 7.3.1. Let $\sigma\left(y^{1}, \pi(T)\right)$ be a $(T \cup\{j\})$-complete $t$-simplex in $A(T)$, $j \notin T$ and let $j_{1}, \ldots, j_{t+1}$ be a permutation of the elements of $I_{t+1}$, such that $\ell\left(y^{j_{t+1}}\right)=j$ and $\ell\left(y^{j_{i}}\right)=s_{i^{\prime}} i=1, \ldots, t$. Then the orientation of $\sigma\left(y^{1}, \pi(T)\right.$ is

$$
\operatorname{or} \sigma=\operatorname{det}\left[\begin{array}{cccc}
1 & \cdots & 1 & 1 \\
\alpha^{j_{1}} & \cdots & \alpha^{j_{t}} & \alpha^{j_{t+1}}
\end{array}\right] \text {. }
$$

Observe that this definition is a straightforwardgeneralization of definition 7.2.7. In particular if $\mathrm{v}=\mathrm{e}(1)$ we have that $S^{\mathrm{n}}(t+1)=A\left(I_{t}\right)$, whereas an $\left(I_{t} U\{j\}\right)$-complete simplex of $S^{n}(t+1)$ must be $I_{t+1}$-complete. of course, or $\sigma= \pm 1$. Definition 7.3 .1 is illustrated in figure 7.3.1.

Remark that the orientation as given in definition 7.3.1 depends on the starting point $v$. In general we have that if the orientation of a $(T \cup\{j\})$-complete simplex is defined for some $v$, it is not defined for a starting point $w \neq v$. For example in the figure, $\tau$ is a $(\{1,2\})$-complete simplex in $A(1)$. However if the point $w=\left(\frac{3}{7}, \frac{2}{7}, \frac{2}{7}\right)^{\top}$ is chosen as the starting point, $\tau_{1}$ is a facet of a simplex in $A(1,3)$ and or $\tau_{1}$ is not defined. Of course the orientation of a completely labelled simplex is always defined by definition 7.3.1 since for any $v$ there exist a $j$ such that $\sigma$ is a completely lahelled simplex of $A\left(I_{n+1} \backslash\{j\}\right)$. We prove that the orientation of a completely labelled simplex as defined in 7.3.1. is independent of the starting point $v$. 


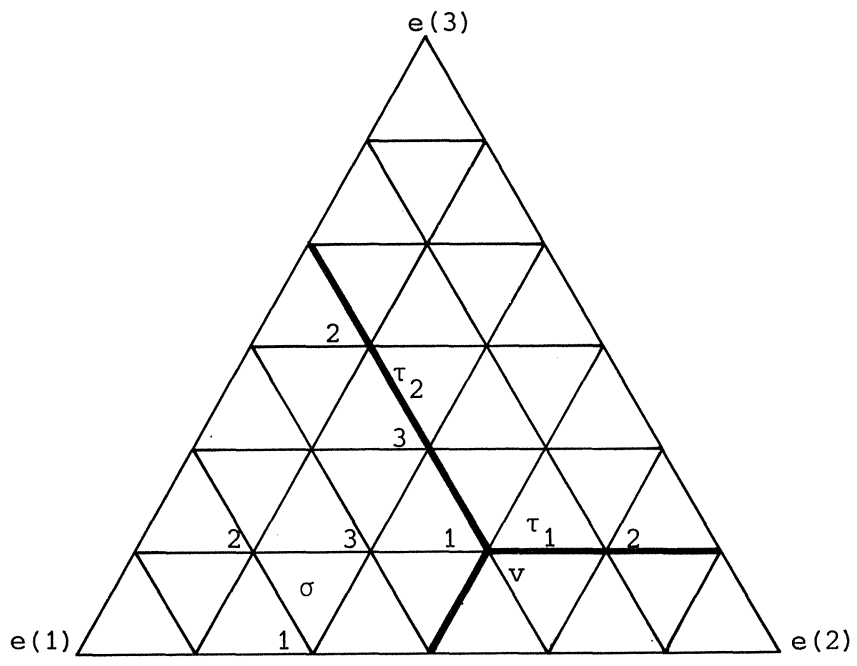

Figure 7.3.1. $\{\mathrm{v}\}$ is a $\{1\}$-complete simplex in $A(\emptyset)$, or $\{v\}=1$; $\tau_{1}$ is a $\{1,2\}$-complete simplex in $A(1)$; or $\tau_{1}=1$; $\tau_{2}$ is a $\{2,3\}$-complete simplex in $A(2)$, or $\tau_{2}=-1$; $\sigma$ is a $\{1,2,3\}$-complete simplex in $A(2,3)$, or $\sigma=-1$.

THEOREM 7.3.2. The orientation of a completely labelled simplex as defined in 7.3.1 is consistent with the orientation as defined in 7.2.1.

PROOF. Let $\sigma\left(y^{1}, \pi(T)\right)$ be a completely labelled simplex in $A(T)$ with $\overline{T=I_{n+1}} \backslash\{k\}$ for some $k \in I_{n+1}$. Let $j_{i}$ be the index such that $\ell\left(y^{j_{i}}\right)=i$, $i=1, \ldots, n+1$. Let $\alpha^{i}=\left(\alpha_{1}^{i}, \ldots, \alpha_{k-1}^{i}, \alpha_{k+1}^{i}, \ldots, \alpha_{n+1}^{i}\right)^{\top}$ be the $n$-vector such that

$$
y^{i}=v+\sum_{j \neq k} \delta \alpha_{j}^{i} q(j) .
$$

Then by definition 7.3.1,

$$
\text { (7.3.2) or } \sigma=\operatorname{det}\left[\begin{array}{cccccccc}
1 & \cdots & 1 & 1 & \cdots & \cdots & 1 & 1 \\
\alpha^{j_{1}} & \cdots & \alpha^{j_{k-1}} & \alpha^{j_{k+1}} & \cdots & \alpha^{j_{n+1}} & \alpha^{j_{k}}
\end{array}\right] \text {. }
$$

We prove that definition 7.2 .1 is equivalent with (7.3.2). From (7.3.1) it follows that for $i=1, \ldots, n+1$, 


$$
\begin{array}{ll}
\mathrm{y}_{\mathrm{h}}^{i}=v_{\mathrm{h}}-\delta \alpha_{\mathrm{h}}^{i}+\delta \alpha_{\mathrm{h}-1}^{i} & \mathrm{~h} \neq \mathrm{k}, \mathrm{k}+1 \\
\mathrm{y}_{\mathrm{h}}^{i}=v_{\mathrm{h}}+\delta \alpha_{\mathrm{h}-1}^{i} & \mathrm{~h}=\mathrm{k} \\
\mathrm{y}_{\mathrm{h}}^{i}=v_{\mathrm{h}}-\delta \alpha_{\mathrm{h}}^{i} & \mathrm{~h}=\mathrm{k}+1
\end{array}
$$

with the convention that $h-1=n+1$ if $h=1$ and $k+1=1$ if $k=n+1$. So the $h-t h$ element of the $i-t h$ column of the matrix $\left[y^{j_{1}} \ldots y^{j_{n+1}}\right]$ becomes

$$
\begin{array}{ll}
\mathrm{v}_{1}-\delta \alpha_{1}^{j_{i}}+\delta \alpha_{h-1}^{j_{i}} & \mathrm{~h}=1 \\
\mathrm{v}_{\mathrm{h}}-\delta \alpha_{\mathrm{h}}^{j_{i}}+\delta \alpha_{\mathrm{h}-1}^{j_{i}} & \mathrm{~h}=2, \ldots, \mathrm{k}-1 \\
\mathrm{v}_{\mathrm{k}}+\delta \alpha_{\mathrm{k}-1}^{j_{i}} & \mathrm{~h}=\mathrm{k} \\
\mathrm{v}_{\mathrm{k}+1}-\delta \alpha_{\mathrm{k}+1}^{j_{i}} & \mathrm{~h}=\mathrm{k}+1 \\
\mathrm{v}_{\mathrm{h}}-\delta \alpha_{\mathrm{h}}^{j_{i}}+\delta \alpha_{\mathrm{h}-1} & \mathrm{~h}=\mathrm{k}+2, \ldots, \mathrm{n}+1 .
\end{array}
$$

By adding successively the $h$-th row to the $(h-1)$-th row, $h=k, k-1, \ldots, 2$, the first row to the last row and then successively the i-th row to the (i-1) -th row, $i=n+1, n, \ldots, k+2$, we obtain that or $\sigma$ is equal to the sign of the determinant of the matrix with the $h$-th element of the $i$-th row equal to

$$
\begin{array}{ll}
\sum_{j=h}^{k} v_{j}+\delta \alpha_{h-1}^{j} & h=1, \ldots, k \\
\sum_{j=1}^{n+1} v_{j}=1 & h=k+1 \\
\sum_{j=1}^{k} v_{j}+\sum_{j=h}^{n+1} v_{j}+\delta \alpha_{h-1}^{j} & h=k+2, \ldots, n+1 .
\end{array}
$$

It follows now immediately that the orientation of $\sigma$ as defined in definition 7.2 .1 becomes 


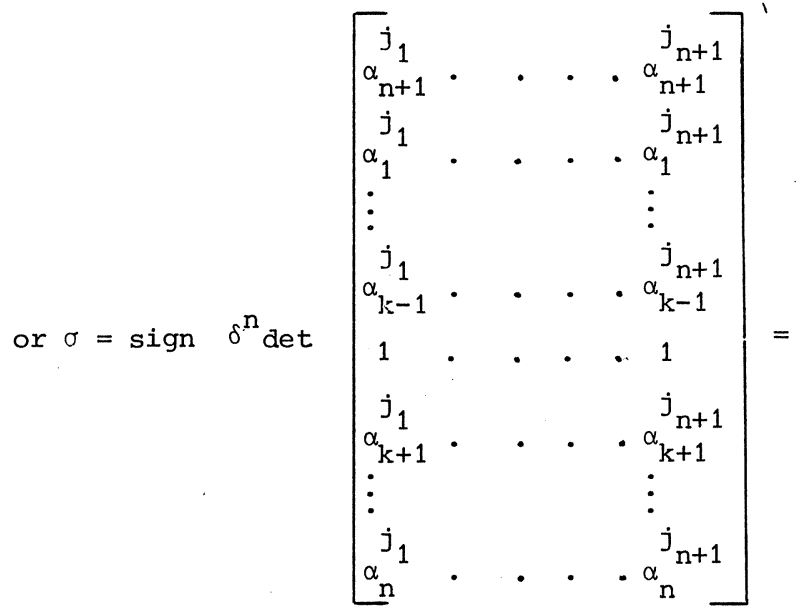

$$
\begin{aligned}
& =\operatorname{det}\left[\begin{array}{ccccccc}
1 & \cdots & 1 & 1 & \cdots & 1 & 1 \\
\alpha^{j_{1}} & \cdots & \alpha^{j_{k-1}} & \alpha^{j_{k+1}} & \ldots & \alpha^{j_{n+1}} & \alpha^{j_{k}}
\end{array}\right] \text {. }
\end{aligned}
$$

Besides the orientation of a $(T U\{j\})$-complete simplex in $A(T)$ we also define the orientation of a $T$-complete facet of a simplex in $A(T)$.

DEFINITION 7.3.3. Let $\tau\left(y^{j_{1}}, \ldots, y^{j_{t}}\right)$ be a $T$-complete facet of a simplex $\sigma\left(y^{1}, \pi(T)\right)$ in $A(T)$ and let the vertices be ordered in such a way that $\ell\left(y^{j}\right)=s_{i}, i=1, \ldots, t$ and $y^{j_{t+1}}$ is the vertex of $\sigma$ opposite to $\tau$. Then the orientation of $\tau$ with respect to $\sigma$ is defined by

$$
\operatorname{or}_{\sigma} \tau=\operatorname{det}\left[\begin{array}{cccc}
1 & \cdots & 1 \\
j^{j_{1}} & & & \alpha_{t+1}^{j_{t+1}}
\end{array}\right] \text {. }
$$

COROLLARY 7.3.4. If $\tau$ is the $T$-complete facet of a ( $T u\{j\})$-complete simplex $\sigma$ in $A(T)$ then or $\sigma=$ or $\sigma$.

The following two lemmas are a straightforward generalization of the lemmas 7.2.9 and 7.2.10. Therefore we omit the proofs of the lemmas.

LEMMA 7.3.5. Let $\sigma\left(y^{1}, \pi(T)\right)$ be a simplex in $A(T)$ having two $T$-complete facets $\tau^{1}$ and $\tau^{2}$. Then

$$
\operatorname{or}_{\sigma} \tau^{1}=-\operatorname{or}_{\sigma} \tau^{2}
$$


LEMMA 7.3.6. Let $\tau$ be a T-complete common facet of two simplices $\sigma_{1}$ and $\sigma_{2}$ in $A(T)$. Then

$$
\operatorname{or}_{\sigma_{1}} \tau=- \text { or }_{\sigma_{2}} \tau
$$

The next lemma is a straightforward generalization of lemma 7.2.11. The proof however has to be adapted since the vertices have to be reordered, if a $(T \cup\{j\})$-complete simplex in $A(T)$ is extended to a simplex in $A(T \cup\{j\})$.

LEMMA 7.3.7. For $|T| \leq n-1$, let $\tau\left(y^{1}, \pi(T)\right)$ be a $(T \cup\{j\})$-complete simplex in $A(T)$. Let $\sigma\left(y^{1}, \pi(T \cup\{j\})\right)$ be the simplex in $A(T \cup\{j\})$ having $\tau$ as a facet. Then

$$
\text { or } \tau=\text { or } \tau \text {. }
$$

PROOF. We have that $y^{i}=v+\Sigma_{s_{h} \in T} \delta \alpha_{s_{h}}^{i} q\left(s_{h}\right), i=1, \ldots, t+1$, whereas the vertex of $\sigma$ opposite to $\tau$ is equal to $y^{t+2}=y_{j}^{t+1}+\delta q(j)$. Let $j_{i}$ be the index such that $\ell\left(y^{j_{i}}\right)=s_{i^{\prime}} i=1, \ldots, t$ and $\ell\left(y^{j_{t+1}}\right)=j$. Then

$$
\text { or } \tau=\operatorname{det}\left[\begin{array}{ccccc}
1 & \cdots & 1 & 1 \\
j_{1} & \cdots & \alpha_{t} & \alpha_{t+1}^{j_{t+1}}
\end{array}\right]=\operatorname{det}\left[\begin{array}{ccccc}
1 & \cdots & \alpha^{1} & 1 \\
\alpha^{j_{1}} & \cdots & \alpha^{j_{t+1}} & \alpha^{t+2} \\
0 & \cdots & 0^{t} & 0
\end{array}\right] \text {. }
$$

Let $i, 0 \leq i \leq t$, be the index such that $s_{1}<j<s_{i+1}$ with the convention that $s_{i}<j$ if $i=t$ and $j<s_{1}$ if $i=0$. Then, by definition 7.3.3, or $\sigma_{\sigma}=$

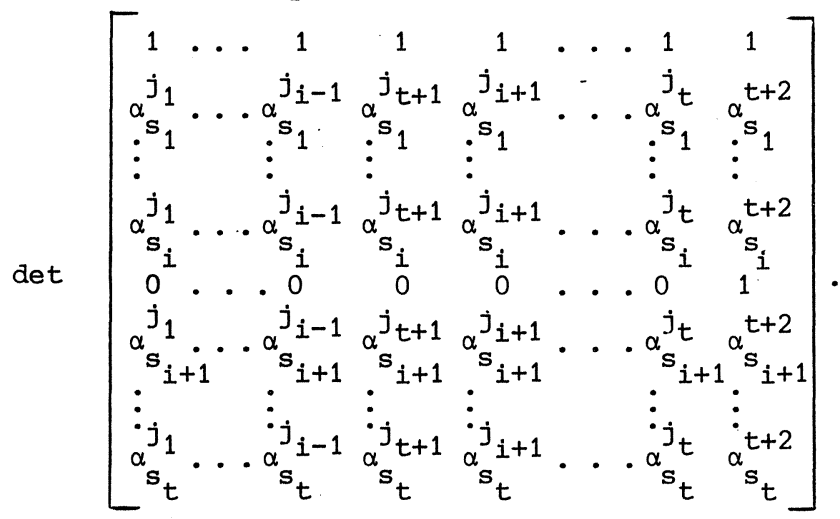

The lemma follows now immediately. 
We give now some results, which can be viewed as a generaliation of theorem 7.2.12. The next theorem states that the sum of the orientations of the ( $T \cup\{j\})$-complete simplices in $A(T)$ is equal to the sum of the orientations of the $T$-complete simplices in $U_{k \in T^{A}}(T \backslash\{k\})$. For a fixed $v$, let $H(T)$ be the set of $(T \cup\{j\})$-complete simplices in $A(T) \quad(j \notin T)$, and let $\bar{H}(T)$ be the set of T-complete simplices in $U_{k \in T^{A}}(T \backslash\{k\})$.

THEOREM 7.3.8. Let the labelling rule be such that the conditions of Sperner's lemma are satisfied. Then

$$
\underset{\sigma \in \mathrm{H}(T)}{\sum} \text { or } \sigma=\sum_{\tau \in \overline{\mathrm{H}}(T)} \text { or } \tau \text {. }
$$

PROOF. The proof of this theorem runs along the lines of the proof of theorem 7.2.12. By applying the variable dimension restart algorithm paths of simplices in $A(T)$ are generated having $T$-complete common facets. Again three cases can happen.

a) Starting with a t-simplex in $A(T)$ having a $T$-complete facet $\tau$ in $A(T \backslash\{k\})$ for some $k \in T, a(T U\{j\})$-complete simplex $\sigma$ in $A(T)$ is generated, or conversely. By corollary 7.3.4 and the lemmas 7.3.5, 7.3.6 and 7.3.7 we have that or $\tau=$ or $\sigma$.

b) Starting with a simplex in $A(T)$ having a $T$-complete facet $\tau_{1}$ in $A\left(T \backslash\left\{k_{1}\right\}\right)$ for some $k_{1} \in T$, a simplex in $A(T)$ is generated having a $T$-complete facet $\tau_{2}$ in $A\left(T \backslash\left\{k_{2}\right\}\right)$ for some $k_{2} \in T$. By the lemmas $7.3 .5-7.3 .7$ we have that or $\tau_{1}=$-or $\tau_{2}$.

c) Starting with a $\left(T u\left\{j_{1}\right\}\right)$-complete simplex $\sigma_{1}$ in $A(T)$ with $j_{1} \notin T$, a ( $\left.T u\left\{j_{2}\right\}\right)$-complete simplex $\sigma_{2}$ in $A(T)$ is generated $\left(j_{2} \notin T\right)$ and from corollary 7.3.4 and the lemmas 7.3.5 and 7.3.6 it follows that or $\sigma_{1}=$-or $\sigma_{2}$.

The theorem follows now immediately. 
Observe that bd $A(T)=\left(b d\left(S^{n}\right) \cap A(T)\right) \cup\left(U_{k \in T^{A}}(T \backslash\{k\})\right)$. Since the labelling rule is proper there are no $T$-complete $(t-1)$-simplices in bd $\left(S^{n}\right) \cap A(T)$. So theorem 7.3.8 states that the sum of the orientations of the T-complete simplices in bd $A(T)$ is equal to the sum of the orientations of the $(T \cup\{j\})$-complete simplices in $A(T)$ if the labelling rule is proper. Assume now that the labelling rule is not proper and let $\tilde{H}(T)$ be the set of the $T$-complete simplices in bd $A(T)$. Let $\tau$ be an element of $\tilde{H}(T)$. If, for some $k \in T, \tau$ is in $A(T \backslash\{k\})$, or $\tau$ is defined by definition 7.3.1. If $\tau$ is in $b d\left(S^{n}\right) \cap A(T)$, let $\sigma$ be the simplex in $A(T)$ such that $\tau$ is a facet of $\sigma$ and define or $\tau$ by or $\tau=$ or $_{\sigma} \tau$ (see definition 7.3.3). Then we have the following corollary, whose proof is analogous to the proof of theorem 7.3 .8 .

COROLLARY 7.3.9. $\sum_{\sigma \in \mathrm{H}(T)}$ or $\sigma=\sum_{\tau \in \tilde{\mathrm{H}}(T)}$ or $\tau$.

THEOREM 7.3.10. Let $T(k)$ be the collection of sets $T \subset I_{n+1}$ such that $|T|=k, k \leq n$. If the labelling rule satisfies the conditions of sperner's lemma, then

$$
\sum_{T \in T(k)} \sum_{\sigma \in \mathrm{H}(T)} \text { or } \sigma=1 \text { for } k=1, \ldots, \mathrm{n} \text {. }
$$

PROOF. Again the proof follows by induction on $k$. For $k=0$ we have that $\{v\}$ is the only $\{l(v)\}$-complete simplex of $A(\phi)$ and $\varnothing$ is the only element of $T(0)$. Moreover, or $\{\mathrm{v}\}=\operatorname{det}[1]=1$. Hence

$$
\sum_{T \in T(0)} \sum_{\sigma \in \mathrm{H}(T)} \text { or } \sigma=\sum_{\sigma \in \mathrm{H}(\varnothing)} \text { or } \sigma=\text { or }\{\mathrm{v}\}=1
$$

Let the statement be true for $k=j$. We prove that it also holds for $k=j+1$. From theorem 7.3 .8 it follows that

$$
\sum_{T \in T(j+1)} \sum_{\sigma \in \mathrm{H}(T)} \text { or } \sigma=\sum_{T \in T(j+1)} \sum_{\tau \in \bar{H}(T)} \text { or } \tau \text {. }
$$

Moreover we have that

$$
\begin{array}{ll}
\frac{\Sigma}{\bar{H}}(T) \\
\text { or } \tau=\sum_{h \in T} \sum_{\sigma \in A(T \backslash\{h\})} \text { or } \sigma . \\
& \sigma \text { is } T \text {-complete }
\end{array}
$$




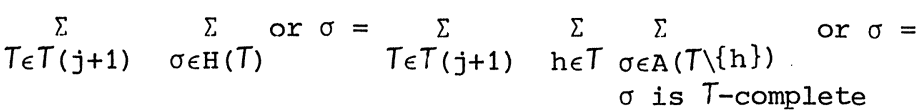

$$
\begin{aligned}
& =\sum_{T \in T(j)} \sum_{i \notin T} \sum_{\sigma \in A(T)} \text { or } \sigma= \\
& \sigma \text { is }(T \cup\{i\}) \text {-complete } \\
& =\sum_{T \in T(j)} \sum_{\sigma \in H(T)} \text { or } \sigma=1 .
\end{aligned}
$$

By taking $\mathrm{v}=\mathrm{e}(1)$ we have that $T=I_{\mathrm{k}}$ is the only feasible element of $T(k)$, i.e. $\stackrel{\circ}{\AA}(T)=\varnothing$ for all $T \in T(k), T \neq I_{k}$. Hence theorem 7.2 .12 is a special case of theorem 7.3.10.

THEOREM 7.3.11. Let $\mathrm{N}\left(\mathrm{S}^{\mathrm{n}}\right)$ be the number of completely labelled simplices in $S^{n}$. Then

$$
\begin{aligned}
& \quad N\left(s^{n}\right) \geq \sum_{j=1}^{n+1}\left|d_{j}\right| \\
& \text { where } d_{j}=\sum_{\sigma \in \tilde{H}\left(I_{n+1} \backslash\{j\}\right)} \text { or } \sigma . \\
& \text { PROOF. With corollary } 7.3 .9 \text { it follows that } \sum_{\sigma \in H\left(I_{n+1} \backslash\{j\}\right)} \text { or } \sigma= \\
& =\sum_{\tau \in \tilde{H}\left(I_{n+1} \backslash\{j\}\right)} \text { or } \tau=d_{j} \text {. Moreover, if } \sigma \in H\left(I_{n+1} \backslash\{j\}\right) \text {, then } \sigma \text { is }
\end{aligned}
$$

completely labelled. Hence the number of completely labelled simplices in $A\left(I_{n+1} \backslash\{j\}\right)$ is at least equal to $\left|d_{j}\right|$. The theorem follows by adding over all $j \in I_{n+1}$.

\subsection{A SEARCH TO AN ODD NUIBER OF COMPLETELY LABELLED SIMPLICES.}

In the previous section we defined the orientation of a (TU\{j\})complete simplex in $A(T)$. However we restricted ourselves to the standard triangulation of $\mathrm{S}^{\mathrm{n}}$. In this section we generalize the definitions 7.3 .1 and 7.3.3 for simplices of a $\mathrm{PK}_{\delta}$ triangulation of $\mathrm{R}^{\mathrm{n}}$. $\mathrm{P}$ is an $\mathrm{n} \times(\mathrm{n}+1)$ triangulation matrix,i.e. $p(n+1)=-\sum_{j=1}^{n} p(j)$ and the $n \times n$ matrix consisting 
of the first $n$ columns of $P$ is nonsingular. A possible choice is $P=U$ (c.f. section 6.2). Recall that the regions $A(T)$ depend on $P$ and are defined by

$$
A(T)=\left\{x \in R^{n} \mid x=v+\sum_{j \in T} \lambda_{j} P(j) \text { for nonnegative numbers } \lambda_{j}, j \in T\right\}
$$

where the grid point $\mathrm{v}$ is the starting point of the variable dimension restart algorithm.

Let $T$ be a subset of $I_{n+1},|T| \leq n_{1}$ and $\left(s_{1}, \ldots, s_{t}\right)$ the permutation of the elements of $T$ such that $s_{j}<s_{j+1}, j=1, \ldots, t-1$. Then the orientation of a $(T \cup\{j\})$-complete simplex $\sigma\left(y^{1}, \pi(T)\right)$ in $A(T)$ and the orientation of a $T$-complete simplex $\tau$ with respect to $\sigma$ where $\tau$ is a facet of the simplex $\sigma\left(y^{1}, \pi(T)\right)$ in $A(T)$, are defined as in the definitions 7.3 .1 and 7.3 .3 respectively, with $\alpha^{i}=\left(\alpha_{s_{1}}^{i}, \ldots, \alpha_{s_{t}}^{i}\right)^{\top}$ the $t$-vector such that

$$
y^{i}=v+\sum_{s_{j} \in T} \delta \alpha_{s_{j}^{i}} p\left(s_{j}\right) \quad i=1, \ldots, t+1 .
$$

THEOREM 7.4.1. For some $T^{*} \subset I_{n+1},\left|T^{*}\right|=n$, let $\sigma\left(y^{1}, \pi\left(T^{*}\right)\right)$ be a completely labelled simplex in $A\left(T^{*}\right)$ generated by the variable dimension restart algorithm. Then or $\sigma=1$.

PROOF. The proof is in fact constructive. Observe that the algorithm starts with the zero-dimensional simplex $\{v\}$ and that or $\{v\}=1$. For some $h$, let $T^{1}, \ldots, T^{h}$ with $T^{1}=\{l(v)\}$ and $T^{h}=T^{*}$ be the sequence of subsets of $I_{n+1}$ such that the algorithm generates $t$-simplices $(t \geq 1)$ in successively $A\left(T^{1}\right), A\left(T^{2}\right), \ldots, A\left(T^{h}\right)$. Define $T^{0}=\varnothing$ and $T^{h+1}=I_{n+1}$. For any $i$, $1 \leq i \leq h$, we have one of the following cases

a) $T^{i-1} \subset T^{i} \subset T^{i+1}$. Then the dimension is increased by changing from $T^{i-1}$ to $T^{i}$ and from $T^{i}$ to $T^{i+1}$. Starting with a simplex in $A\left(T^{i}\right)$ having a $T^{i}$-complete facet $\tau$ in $A\left(T^{i-1}\right)$, a $T^{i+1}$-complete simplex $\sigma$ of $A\left(T^{i}\right)$ is generated. By corollary 7.3 .4 and the lemas $7.3 .5,7.3 .6$ and 7.3.7, we have that or $\tau=$ or $\sigma$ (If $i=h$, the dimension is not increased).

b) $T^{i-1} \subset T^{i}$ and $T^{i+1} \subset T^{i}$. Then the dimension is increased by changing from $T^{i-1}$ to $T^{i}$ and decreased by changing from $T^{i}$ to $T^{i+1}$. Starting with a simplex in $A\left(T^{i}\right)$ having a $T^{i}$-complete facet $\tau_{1}$ in $A\left(T^{i-1}\right)$, a simplex in $A\left(T^{i}\right)$ is generated having a $T^{i}$-complete facet $\tau_{2}$ in $A\left(T^{i+1}\right)$. 
By the lemmas $7 \cdot 3 \cdot 5 \cdot-7 \cdot 3 \cdot 7$, we have that or $\tau_{2}=$-or $\tau_{1}$.

c) $T^{i+1} \subset T^{i} \subset T^{i-1}$. The dimension is decreased by changing from $T^{i-1}$ to $T^{i}$ and from $T^{i}$ to $T^{i+1}$. This case is the reverse of case a. Starting with a $T^{i-1}$-complete simplex $\sigma$ in $A\left(T^{i}\right)$ a simplex in $A\left(T^{i}\right)$ is generated having a $T^{i}$-complete facet $\tau$ in $A\left(T^{i+1}\right)$. Again we have that or $\sigma=$ or $\tau$.

d) $T^{i} \subset T^{i-1}$ and $T^{i} \subset T^{i+1}$. The dimension is decreased by changing from $T^{i-1}$ to $T^{i}$ and increased by changing from $T^{i}$ to $T^{i+1}$. Starting with a $T^{i-1}$-complete simplex $\sigma_{1}$ in $A\left(T^{i}\right)$, a $T^{i+1}$-complete simplex $\sigma_{2}$ in $A\left(T^{i}\right)$ is generated. From corollary 7.3.4 and the lemmas 7.3.5 and 7.3.6, it follows that or $\sigma_{2}=-$ or $\sigma_{1}$.

Observe that the orientation changes in the cases $b$ and $d$. Let $i_{1}, \ldots, i_{r}$ with $i_{j}<i_{j+1}, j=1, \ldots, r-1$, be the sequence of indices for which case $b$ occurs. Then we must have a sequence of indices $h_{1}, \ldots, h_{r}$ with $i_{j}<h_{j}<i_{j+1}$, $j=1, \ldots, r-1$ and $h_{r}>i_{r}$ for which case $d$ occurs. By these arguments it follows that or $\sigma=\operatorname{or}\{v\}=1$.

Let $P^{1}$ and $P^{2}$ be two $n \times(n+1)$ triangulation matrices such that for some $i_{1}$ and $i_{2}$ holds

$$
\left\{\begin{array}{l}
p^{1}(j)=p^{2}(j) \quad j \neq i_{1}, i_{2} \\
p^{1}\left(i_{1}\right)=p^{2}\left(i_{2}\right) \quad \text { and } p^{1}\left(i_{2}\right)=p^{2}\left(i_{1}\right)
\end{array}\right.
$$

where $p^{h}(j)$ is the $j$-th column of $p^{h}, h=1,2$.

So $P^{2}$ is obtained from $P^{1}$ by interchanging the columns $i_{1}$ and $i_{2}$. Clearly, each simplex of the $\mathrm{P}^{1} \mathrm{~K}_{\delta}$ triangulation is a simplex of the $\mathrm{P}^{2} \mathrm{~K}_{\delta}$ triangulation and conversely. The next theorem states that the sign of the orientation of a completely labelled simplex changes if two columns of the triangulation matrix are interchanged.

THEOREM 7.4.2. Let $\mathrm{P}^{1}$ and $\mathrm{P}^{2}$ be two $\mathrm{n} \times(\mathrm{n}+1)$ triangulation matrices such that (7.4.1) holds. Let $\sigma$ be a completely labelled simplex and let or ${ }^{\mathrm{h}}$ be the orientation of $\sigma$ if $\mathrm{P}^{\mathrm{h}}$ is the triangulation matrix, $h=1,2$. Then 


$$
\operatorname{or}^{1} \sigma=-\mathrm{or}^{2} \sigma \text {. }
$$

PROOF. In theorem 7.3.2 it was proved that the orientation of a completely labelled simplex, as defined in definition 7.3.1, of the standard triangulation of $\mathrm{s}^{\mathrm{n}}$ is independent of $\mathrm{v}$. It can be easily verified that this theorem also holds for a completely labelled simplex of a $\mathrm{PK}_{\delta}$ triangulation of $R^{n}$. Therefore we can choose $v$ such that $\sigma$ is in some region $A(T) \quad(|T|=n)$ with $i_{1}$ and $i_{2}$ both an element of $T$. For some vertex $y^{i}$ of $\sigma$ let $\alpha^{h i}=\left(\alpha_{s_{1}}^{h i}, \ldots, \alpha_{s_{n}}\right)^{T}$ be the vector such that

$$
y^{i}=v+\sum_{s_{j} \in T} \delta \alpha_{s_{j}}^{h i} p^{h}\left(s_{j}\right) \quad h=1,2 .
$$

Clearly, for all $i, i=1, \ldots, n+1, \alpha_{j}^{1 i}=\alpha{ }_{j}^{2 i}$ for all $j \neq i_{1}, i_{2}$ whereas $\alpha_{i_{1}}^{1 i}=\alpha \frac{2 i}{i_{2}}$ and $\alpha_{i_{2}}^{1 i}=\alpha_{i_{1}}^{2 i}$. Hence $\alpha^{2 i}$ is obtained from $\alpha^{1 i}$ by interchanging two elements. So, with $j_{1}, \ldots, j_{n+1}$ such that $\ell\left(y^{j_{i}}\right)=s_{i}, 1 \leq i \leq n$ and $\ell\left(y^{j_{n+1}}\right)=h, h \notin T$,

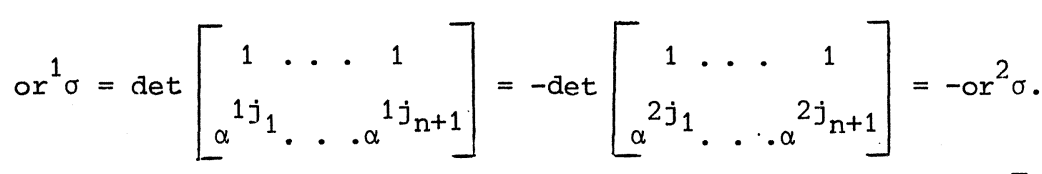

Now we have a tool to generate more completely labelled simplices (if exist) by using two matrices $\mathrm{P}^{1}$ and $\mathrm{P}^{2}$ such that (7.4.1) holds. Assume that the variable dimension restart algorithm generates a completely labelled simplex $\sigma_{1}$ with $\mathrm{P}^{1} \mathrm{~K}_{\delta}$ the underlying triangulation. Clearly by theorem 7.4.1 we have that or ${ }^{1} \sigma_{1}=1$ and by theorem 7.4.2 or ${ }^{2} \sigma_{1}=-1$. Let the variable dimension restart algorithm now be applied with $\mathrm{P}^{2} \mathrm{~K}_{\delta}$ as underlying triangulation. Then either a path of simplices going to infinity is generated or a path which terminates with a completely labelled simplex $\sigma_{2}$. Clearly or ${ }^{2} \sigma_{2}=1$ and hence $\sigma_{2} \neq \sigma_{1}$. Moreover, a search for a third completely labelled simplex can be made. Therefore, let $T_{j}^{\mathrm{h}}$ $\left(\left|T_{j}^{\mathrm{h}}\right|=n\right)$ be the set of indices such that $\sigma_{j} \in A\left(T_{j}^{\mathrm{h}}\right)$ if $\mathrm{P}^{\mathrm{h}} \mathrm{K}_{\delta}$ is the underlying triangulation, $h=1,2$. So

$$
\begin{aligned}
& T_{j}^{2}=T_{j}^{1} \text { if } i_{1}, i_{2} \in T_{j}^{1} \\
& T_{j}^{2}=\left(T_{j}^{1} \cup\left\{i_{2}\right\}\right) \backslash\left\{i_{1}\right\} \text { if } i_{2} \notin T_{j}^{1}
\end{aligned}
$$




$$
T_{j}^{2}=\left(T_{j}^{1} \cup\left\{i_{1}\right\}\right) \backslash\left\{i_{2}\right\} \text { if } i_{1} \notin T_{j}^{1} .
$$

Now using the $\mathrm{P}^{2} \mathrm{~K}_{\delta}$ triangulation and starting with the completely labelled simplex $\sigma_{1}$ by replacing the vertex with the label $\mathrm{k}, \mathrm{k} \notin T_{1}^{2}$, the algorithm generates a sequence of simplices of variable dimension such that the common facet of two adjacent simplices in $A(T)$ is $T$-complete. Clearly, by theorem 7.4.1 this sequence of simplices cannot terminate with the zero-dimensional simplex $\{\mathrm{v}\}$ since or $^{2} \sigma_{1}=-1$. Hence either a sequence of simplices going to infinity is generated or the algorithm terminates with a completely labelled simplex $\sigma_{3} \neq \sigma_{1}$. Using analogous arguments as in the proof of theorem 7.4.1, it follows that or $^{2} \sigma_{3}=-\operatorname{or}^{2} \sigma_{1}=1$. However using the standard arguments, $\sigma_{3} \neq \sigma_{2}$, since there exists a path of simplices which starts with $\{\mathrm{v}\}$ and terminates with $\sigma_{2}$. Therefore three completely labelled simplices are generated. The method is illustrated in the figures 7.4.1 and 7.4.2. We have that

$$
P^{1}=\left[\begin{array}{lll}
1 & 0 & -1 \\
0 & 1 & -1
\end{array}\right] \text { and } P^{2}=\left[\begin{array}{ccc}
0 & 1 & -1 \\
1 & 0 & -1
\end{array}\right] \text {. }
$$

For $i=1,2$, we define $A^{i}(T)$ by

$$
A^{i}(T)=\left\{x \in R^{n} \mid x=v+\sum_{j \in T} \lambda_{j} p^{i}(j) \text { for nonnegative numbers } \lambda_{j}, j \in T\right\} .
$$

In figure 7.4.1 the regions $A^{1}(T)$ are given. Starting in $v$ and using the $\mathrm{P}^{1} \mathrm{~K}$ triangulation the completely labelled simolex $\sigma_{1}$ is generated. Observe that $T_{1}^{1}=\{1,3\}$. Hence $T_{1}^{2}=\{1,3\} \cup\{2\} /\{1\}=\{2, \overline{3}\}$. Using the $\mathrm{P}^{2} \mathrm{~K}$ triangulation and starting with $\sigma_{1}$ by replacing the vertex having label 1 , the algorithm generates the completely labelled simplex $\sigma_{3}$, as is illustrated in figure 7.4.2. Moreover, starting from $v$ the completely labelled simplex $\sigma_{2}$ is generated.

We give now a condition for the existence of at least $\mathrm{k}$ completely labelled simplices, $k=1,3,5, \ldots$.

THEOREM 7.4.3. Let $\mathrm{f}$ be a continuous function on $\mathrm{R}^{\mathrm{n}}$. Assume that for some integer $r$ there exists an increasing sequence of positive numbers 


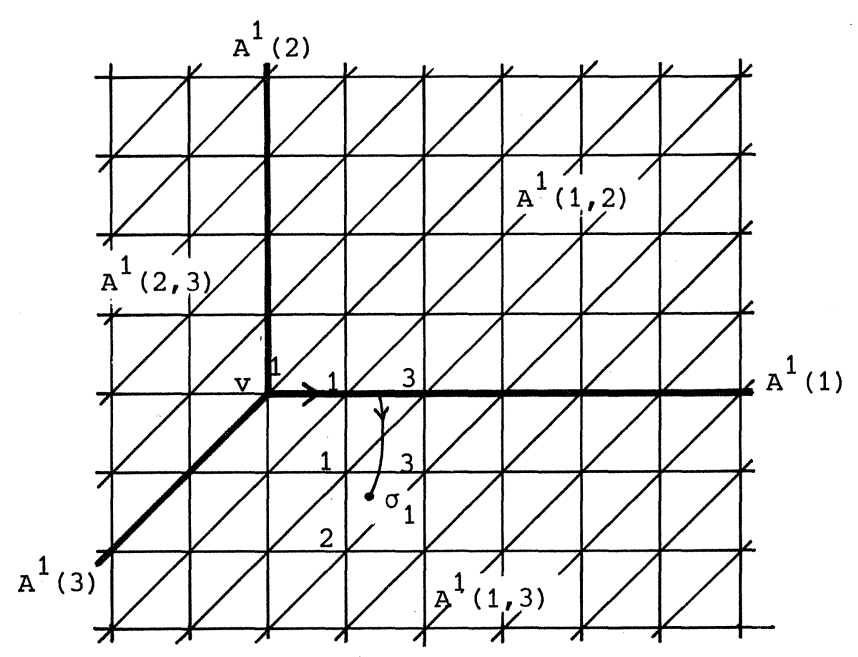

Figure 7.4.1. Using the $\mathrm{P}^{1} \mathrm{~K}$ triangulation $\sigma_{1}$ is generated.

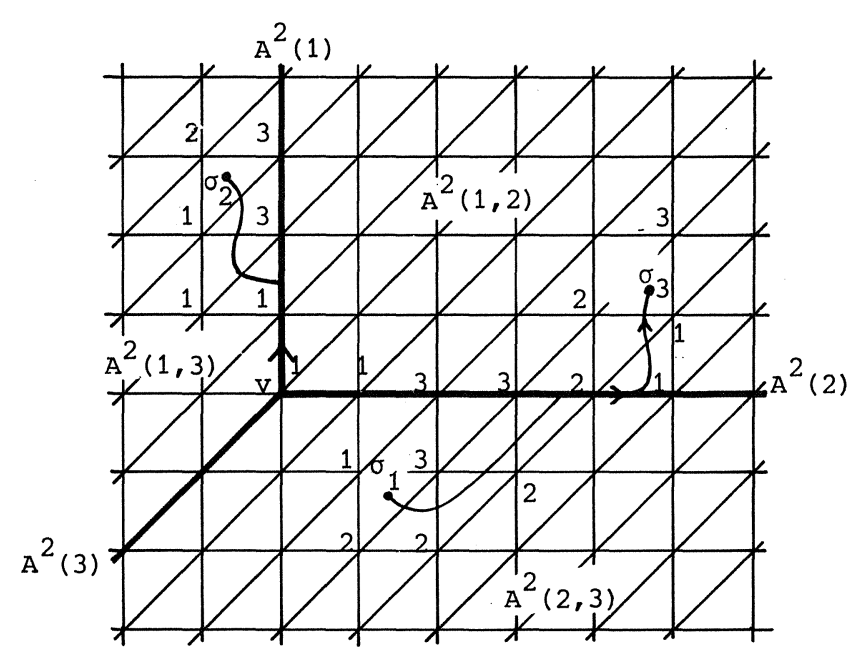

Figure 7.4.2. Using the $\mathrm{P}^{2} \mathrm{~K}$ triangulation $\sigma_{2}$ and $\sigma_{3}$ are generated. 
$\mu_{1}, \mu_{2}, \ldots, \mu_{r}$ and that there exist a number $\varepsilon>0$ and two indices $i_{1}, i_{2}$, such that for all $x \in \tilde{B}^{n}\left(\mu_{h}\right), h=1,2, \ldots, r$, holds that

or

a) For some $i, x_{i}=-\mu_{h}$ and $f_{j}(x)-x_{j}>\varepsilon$ for at least one index $j \in I_{n}$

b) For some $i, x_{i}=\mu_{h}$ and we have that

or

(i) $f_{j}(x)-x_{j}<-\varepsilon$ for all $j \in I_{n}$

(ii) there exists at least one index $j, j \neq s$, with $f_{j}(x)-x_{j}>f_{s}(x)-x_{s}+\varepsilon$, where $s=i$ in the case that $h$ is odd and, if $h$ is even, $s=i$ when $i \neq i_{1}, i_{2}$, $s=i_{2}$ when $i=i_{1}$ and $s=i_{1}$ when $i=i_{2}$.

Let $R^{n}$ be triangulated by $k_{\delta}$. Then, if $\delta$ is small enough, at least $2 h-1$ completely labelled simplices in $\operatorname{conv}\left(\tilde{B}^{n}\left(\mu_{h}\right)\right), h=1,2, \ldots, r$, can be generated by applying the variable dimension restart algorithm.

PROOF. Let $\mathrm{P}^{1}$ be the $\mathrm{n} \times(\mathrm{n}+1)$ matrix with $\underline{p}^{1}(i)=e(i), i \in I_{n}$ and $\mathrm{p}^{1}(\mathrm{n}+1)=-e$ and $\mathrm{P}^{2}$ the $\mathrm{n} \times(n+1)$ matrix with $\mathrm{p}^{2}(i)=\mathrm{p}^{1}(i)$ for $i_{\neq} \mathrm{i}_{1}, i_{2}$, $p^{2}\left(i_{1}\right)=p^{1}\left(i_{2}\right)$ and $p^{2}\left(i_{2}\right)=p^{1}\left(i_{1}\right)$. Clearly, both matrices induce the $k$ triangulation of $R^{n}$. Let the grid size $\delta$ be so small that

$$
\sup _{(x, y) \in \sigma} \max _{i \in I_{n}}\left|f_{i}(x)-x_{i}-f_{i}(y)+y_{i}\right|<\frac{1}{2} \varepsilon
$$

for every simplex $\sigma$ such that $\sigma \cap \tilde{B}^{n}\left(\mu_{h}\right) \neq \varnothing$ for some $h \in I_{x}$. Clearly, if $h$ is odd, the conditions of theorem 6.3.2 are satisfied. So with $\mathrm{P}^{1} \mathrm{~K}_{\delta}$ as underlying triangulation and starting in $\operatorname{conv}\left(\tilde{B}^{n}\left(\mu_{h}\right)\right)$ the algorithm generates a completely labelled simplex in $\operatorname{conv}\left(\tilde{B}^{n}\left(\mu_{h}\right)\right), h=1,3,5, \ldots, r$ (or $\left.r-1\right)$. However recall, that the convergence proof is based on the fact that if $\sigma\left(w^{1}, \ldots, w^{t+1}\right)$ is a simplex in $A(T)$ and $\sigma \cap \tilde{B}^{n}\left(\mu_{h}\right) \neq \varnothing$, there exists an index $i \in T$ such that $\ell\left(w^{k}\right) \neq i, k=1, \ldots, t+1$. It can be easily verified that this argument also holds if $h$ is even with $\mathrm{P}^{2} \mathrm{~K}_{\delta}$ the underlying triangulation. Hence, starting in $\operatorname{conv}\left(\tilde{B}^{\tilde{r}_{1}}\left(\mu_{h}\right)\right)$ and with $P^{i} K_{\delta}$ the underlying triangulation, where $i=2-h(\bmod 2)$, the algorithm generates a 
completely labelling simplex in $\operatorname{conv}\left(\tilde{B}^{n}\left(\mu_{h}\right)\right)$. Now the theorem follows by induction on $h$. Starting in a grid point $v$ in $\operatorname{conv}\left(\tilde{B}^{n}\left(\mu_{1}\right)\right)$ and using the $\mathrm{P}^{1} \mathrm{~K}_{\delta}$ triangulation the algorithm generates a completely labelled simplex $\sigma_{1}$ in conv $\left(\tilde{B}^{n}\left(\mu_{1}\right)\right)$. Clearly $v$ and $\sigma_{1}$ are in conv $\left(\tilde{B}^{n}\left(\mu_{2}\right)\right)$. Hence using the $P^{2} K_{\delta}$ triangulation and starting with $v$ respectively $\sigma_{1}$ the algorithm generates completely labelled simplices $\sigma_{2}$ and $\sigma_{3}$ in $\operatorname{conv}\left(\tilde{B}^{\mathrm{n}}\left(\mu_{2}\right)\right)$ with, as argued above, $\sigma_{i} \neq \sigma_{j}$ if $i \neq j$.

Now, let $\sigma_{2 \mathrm{k}-2}$ and $\sigma_{2 \mathrm{k}-1}$ be the completely labelled simplices in $\operatorname{conv}\left(\tilde{B}^{\mathrm{n}}\left(\mu_{\mathrm{k}}\right)\right)$ found by the algorithm. Clearly $\sigma_{2 \mathrm{k}-2}$ and $\sigma_{2 \mathrm{k}-1}$ are in $\operatorname{conv}\left(\tilde{B}^{1}\left(\mu_{k+1}\right)\right)$. Now using the $P^{i} K_{\delta}$ triangulation where $i=k(\bmod 2)+1$, and starting with $\sigma_{2 \mathrm{k}-2}$ respectively $\sigma_{2 \mathrm{k}-1}$ as described above, the algorithm generates two completely labelled simplices $\sigma_{2 k}$ and $\sigma_{2 k+1}$ in $\operatorname{conv}\left(\tilde{B}^{n}\left(\mu_{k+1}\right)\right)$. If $k+1$ is odd we have that there exist paths of simplices between $\{\mathrm{v}\}$ and $\sigma_{1}, \sigma_{4 h-2}$ and $\sigma_{4 h}, h=1, \ldots, \frac{1}{2} k-1$, and $\sigma_{4 h-1}$ and $\sigma_{4 h+1}, h=1, \ldots,,_{\frac{1}{2} k-1}$. Hence $\sigma_{2 k} \neq \sigma_{i}, i<2 k$, and $\sigma_{2 k+1} \neq \sigma_{i}, i<2 k+1$. If $k+1$ is even we have that there exist paths of simplices between $\{\mathrm{v}\}$ and $\sigma_{2}, \sigma_{4 \mathrm{~h}}$ and $\sigma_{4 \mathrm{~h}+2}$ ' $h=1, \ldots, \frac{1}{2}(k-3)$ and $\sigma_{4 h-3}$ and $\sigma_{4 h-1}, h=1, \ldots, \frac{1}{2}(k-1)$, which again implies that $\sigma_{2 k} \neq \sigma_{i^{\prime}} i<2 k$, and $\sigma_{2 k+1} \neq \sigma_{i^{\prime}} i<2 k+1$. Hence, for $h=1, \ldots, r$, at least $2 \mathrm{~h}-1$ completely labelled simplices can be generated in $\operatorname{conv}\left(\tilde{B}^{\mathrm{n}}\left(\mu_{h}\right)\right)$.

COROLLARY 7.4.4. If the conditions of theorem 7.4.3 are satisfied there exist at least $2 \mathrm{~h}-1$ completely labelled simplices in $\operatorname{conv}\left(\tilde{B}^{n}\left(\mu_{h}\right)\right), h=1, \ldots, r$.

Note that the conditions of theorem 7.4.3 can be easily modified. For example the theorem is also true if in $b(i i)$ the condition for $h$ is even and $\mathrm{h}$ is odd interchanged. Approximating fixed points, it is worthwile to investigate if the function satisfies conditions similar to those of theorem 7.4.3. Finally, we remark that a condition for the existence of two fixed points is given by Allgower and Georg [1980]. They present also a simplicial algorithm to approximate both points. The algorithm is based on an idea of Jeppson [1972]. 
CHAPTER 8

A CONTINUOUS DEFORMATION ALGORITHM WITH ARBITRARY REFINEMENT FACTORS

\subsection{INTRODUCTION.}

In chapter 4 we discussed the continuous deformation algorithm developed by Eaves [1972] and Eaves and Saigal [1972], to compute a fixed point of a function or mapping. These authors constructed a triangulation of $S^{n} \times[1, \infty)$ respectively $R^{n} \times[1, \infty)$. Restricting ourselves to the first set, $s^{n} \times\left\{d_{k}\right\}$ was triangulated with grid size $d_{k}^{-1}$ by the standard triangulation, where $d_{0}, d_{1}, \ldots$ is an increasing sequence of integers with $d_{0}=1$. Then for each $i, i=0,1, \ldots, s^{n} \times\left[d_{i}, d_{i+1}\right]$ was triangulated by $(n+1)$-simplices such that the vertices of these simplices are grid points in $s^{n} \times\left\{d_{i}\right\}$ or $s^{n} \times\left\{d_{i+1}\right\}$. Until recently, only triangulations were known such that $a_{i+1} / d_{i} \leq 2$ for all $i$ (see Todd $\left.[1976 a]\right)$. This restriction is a serious drawback in comparison with restart methods.

In this chapter, which is based on Van der Laan and Talman [1980b] we present a continuous deformation algorithm which allows for an arbitrarily chosen factor of incrementation $k_{i}=d_{i+1} / d_{i}, i \geq 0$. In chapter 5 we showed how the variable dimension restart algorithm can be interpreted as an algorithm with $\mathrm{n}+1$ points on an extra level. In this interpretation $s^{n} \times\{0\}$ is triangulated with grid size one, $s^{n} \times\{1\}$ with an arbitrarily chosen grid size $\mathrm{m}^{-1}$ and $s^{\mathrm{n}} \times[0,1]$ is triangulated by $\mathrm{n}+1$ simplices such that all vertices are grid points in $s^{n} \times\{0\}$ or $s^{n} \times\{1\}$. Van der Laan and Talman used this triangulation of $s^{n} \times[0,1]$ to construct a triangulation of $s^{n} \times\left[1, \infty\right.$; such that the factors $d_{i+1} / d_{i^{\prime}} i=0,1, \ldots$, are arbitrary integers larger than one. Using this triangulation in a fixed point algorithm the levels $d_{i}$ can be chosen throughout the algorithm, i.e. $d_{m+1}$ is chosen as soon as a completely labelled simplex $\sigma_{m}$ on level $d_{m}$ is found. Moreover we have that the grid point on level $d_{m+1}$ connected with the vertices of $\sigma_{\mathrm{m}}$ on level $\mathrm{d}_{\mathrm{m}}$ is an arbitrarily chosen grid point $\mathrm{v}\left(\sigma_{\mathrm{m}}\right)$ of 
$\sigma_{m} \times\left\{d_{m+1}\right\}$. Of course, the choices of $d_{m+1}$ and $v\left(\sigma_{m}\right)$ are done on ground of the information obtained from the completely labelled simplex $\sigma_{m} \times\left\{d_{m}\right\}$. As soon as they are chosen, the triangulation of $s^{n} \times\left[d_{m}, d_{m+1}\right]$ is fixed throughout the remaining of the algorithm.

In section 2 the triangulation of $S^{n} \times[1, \infty)$ is described. In section 3 we give a concise description of the algorithm, whereas the replacement steps are given in section 4 . In section 5 we discuss the application on $\mathrm{R}^{\mathrm{n}}$. In section 6 some remarks are made and some numerical results are presenter.

\subsection{TRIANGULATION OF $S^{\mathrm{n}} \times[1, \infty)$.}

Let $a_{0}, d_{1}, \ldots$ be a sequence of increasing integers such that $d_{0}=1$ and $d_{i+1}=k_{i} d_{i}, i=0,1, \ldots$, with $k_{i}$ an arbitrary integer larger than 1 . In the following we triangulate, for some $m$, the set $s^{n} \times\left[d_{m}, d_{m+1}\right]$ such that all grid points arepoints of $s^{n} \times\left\{d_{m}\right\}$ or $s^{n} \times\left\{d_{m+1}\right\}$. Combining the triangulations of $s^{n} \times\left[d_{i}, d_{i+1}\right]$ over all pairs $\left(d_{i}, d_{i+1}\right)$ we obtain a triangulation of $s^{n} \times[1, \infty)$. For $i=0,1, \ldots$ let $G_{i}$ be the standard triangulation of $S^{n}$ with grid size $d_{i}^{-1}$. So, by the definitions 3.4 .1 and 5.2.1, $G_{m}$ is the collection of n-simplices $\sigma\left(y^{1}, \pi(T)\right) \quad\left(T \subset I_{n+1},|T|=n\right)$ with vertices $\mathrm{y}^{1}, \ldots, \mathrm{y}^{\mathrm{n}+1}$ such that

a) the components of $y^{1}$ are a multiple of $d_{m}^{-1}$

b) $\pi(T)=\left(\pi_{1}, \ldots, \pi_{n}\right)$ is a permutation of the elements of $T$

c) $y^{i+1}=y^{i}+q\left(\pi_{i}\right) / d_{m} \quad i=1, \ldots, n$,

where $q(j)$ is the $j$-th column of the $(n+1) \times(n+1)$ matrix defined in section 5.2. Observe that $y^{1}=y^{n+1}+q\left(\pi_{n+1}\right) / d_{m}$ where $\pi_{n+1}$ is the element of $I_{n+1}$ not in $T$. Therefore, in the following an n-simplex $\sigma\left(\mathrm{y}^{1}, \pi(T)\right)$ is also denoted by $\sigma\left(y^{1}, \pi\left(I_{n+1}\right)\right)$ where $\pi\left(I_{n+1}\right)=\left(\pi(T), \pi_{n+1}\right)$ with $\left\{\pi_{n+1}\right\}=I_{n+1} \backslash T$. As pointed out in section 5.2 we have that every simplex has $n+1$ representations, since each vertex can be chosen to be $\mathrm{y}^{1}$. However, it will appear that it is more appropriate to represent an n-simplex in a unique way. This can be done as follows (see also Eaves and Saigal [1972]). For a given grid point w of $G_{m^{\prime}}$ let $\alpha(w)=\left(\alpha_{1}(w), \ldots, \alpha_{n}(w)\right)^{\top}$ be the n-vector with

$$
\alpha_{h}(w)=\left(1-\sum_{j=1}^{h} w_{j}\right) d_{m^{\prime}} \quad h=1, \ldots, n \text {. }
$$

Clearly, every $\alpha_{h}(w)$ is an integer. Now we define a "labelling function" $s$ from the set of grid points of $G_{m}$ to the set $I_{n+1}$ by 


$$
s(w)=1+\left(\sum_{h=1}^{n} \alpha_{h}(w)\right) \bmod (n+1)
$$

LEMMA 8.2.1. Let $\sigma\left(\mathrm{y}^{1}, \pi\left(I_{\mathrm{n}+1}\right)\right)$ be an $\mathrm{n}$-simplex of $\mathrm{G}_{\mathrm{m}}$. Then, for all $i \in I_{n+1}$, there exists an index $j_{i}$ such that $s\left(y^{j}\right)=i$.

PROOF. We prove that for each $j, j=1, \ldots, n$,

$$
s\left(y^{j+1}\right)=s\left(y^{j}\right) \bmod (n+1)+1
$$

Then the lemma follows immediately. For some $j$, let $s\left(y^{j}\right)=i$. First suppose that $\pi_{j} \neq n+1$. Then $y_{h}^{j+1}=y_{h^{\prime}}^{j}, h \neq \pi_{j}, \pi_{j}+1, y_{h}^{j+1}=y_{h}^{j}-d_{m}^{-1} \quad h=\pi_{j}$ and $y_{h}^{j+1}=y_{h}^{j}+d_{m}^{-1}$, $h=\pi_{j}+1$. Hence $\alpha_{h}\left(y^{j+1}\right)=\alpha_{h}\left(y^{j}\right)$ for $h \neq \pi_{j}$ and $\alpha_{h}\left(y^{j+1}\right)=\alpha_{h}\left(y^{j}\right)+1$ for $h=\pi_{j}$. Therefore $\Sigma_{h=1}^{n} \alpha_{h}\left(y^{j+1}\right)=1+\Sigma_{h=1}^{n} \alpha_{h}\left(y^{j}\right)$, which proves that (8.2.1) holds. If $\pi_{j}=n+1$ we have that $y_{h}^{j+1}=y_{h^{\prime}}^{j}, h \neq 1, n+1, y_{1}^{j+1}=y_{1}^{j}+d_{m}^{-1}$ and $y_{n+1}^{j+1}=y_{n+1}^{j}-d_{m}^{-1}$. Hence $\alpha_{h}\left(y^{j+1}\right)=\alpha_{h}\left(y^{j}\right)-1, h=1, \ldots, n$, and therefore $\sum_{h=1}^{n} \alpha_{h}\left(y^{j+1}\right)=$ $=\sum_{h=1}^{n} \alpha_{h}\left(y^{j}\right)+1-(n+1)$, which again proves that (8.2.1) holds.

The lemma means that each simplex is "completely labelled" with respect to the function $s(w)$. Moreover if $s\left(y^{1}\right)=1$ we have that $s\left(y^{j}\right)=j, j=1, \ldots, n+1$. In the following we assume that every n-simplex $\sigma\left(y^{1}, \pi\left(I_{n+1}\right)\right)$ of $G_{m}$ is represented in such a way that $s\left(\mathrm{y}^{1}\right)=1$. This representation is called the s-representation of a simplex $\sigma$. Let $\sigma_{1}\left(y^{1}, \pi\left(I_{n+1}\right)\right)$ have the s-representation and let $\sigma_{2}\left(\bar{y}^{-1}, \bar{\pi}\left(I_{n+1}\right)\right)$ be the adjacent simplex, obtained from $\sigma_{1}$ by replacing $y^{i}$. Then $\sigma_{2}$ has also the s-representation if $y^{i}$ is replaced according to table 8.2.1. This can easily be seen by observing that $\bar{y}^{-h}=y \quad h \neq i$. Hence $s\left(\bar{y}^{h}\right)=s\left(y^{h}\right), h \neq i$ and therefore also $s\left(\bar{y}^{i}\right)=s\left(y^{i}\right)$.

T'able 8.2.1. $\mathrm{s}$ is the index of the vertex to be replaced.

\begin{tabular}{l|l|l} 
& $y^{1}$ becomes & $\pi_{n+1}\left(I_{n}\right)$ becomes \\
\hline$s=1$ & $y^{n+1}+q\left(\pi_{1}\right) / d_{m}$ & $\begin{array}{l}\left(\pi_{n+1}, \pi_{2}, \ldots, \pi_{n}, \pi_{1}\right) \\
\left(\pi_{1}, \ldots, \pi_{s-2}, \pi_{s}, \pi_{s-1}, \pi_{s+1}, \ldots, \pi_{n+1}\right)\end{array}$
\end{tabular}


We are now ready to triangulate $s^{n} \times\left[d_{m}, d_{m+1}\right]$ for some given $m$. First we take a particular simplex $\sigma_{m}\left(u^{1}, \beta\left(I_{n+1}\right)\right)$ of the triangulation $G_{m}$ called the starting simplex on level $d_{m}$, with $u^{1}$ and $\beta\left(I_{n+1}\right)$ chosen in such a way that we have the s-representation of $\sigma_{m}$. Then we choose nonnegative integers $\lambda_{1}^{\mathrm{m}}, \ldots, \lambda_{\mathrm{n}+1}^{\mathrm{m}}$ with sum equal to $\mathrm{k}_{\mathrm{m}}=\mathrm{d}_{\mathrm{m}+1} / \mathrm{d}_{\mathrm{m}}$ and we call the point

$$
v\left(\sigma_{m}\right)=\sum_{i=1}^{n+1} k_{m}^{-1} \lambda_{i}^{m} u^{i}
$$

the centrepoint of $\sigma_{m}$. Observe that $v\left(\sigma_{m}\right)$ is a grid point of $G_{m+1}$. Applying the continuous deformation algorithm to approximate a fixed point of a function or mapping, $\sigma_{\mathrm{m}}$ is the first completely labelled $n$-simplex generated by the algorithm on level $d_{m}$ and $\lambda_{i^{\prime}}^{m} 0 \leq i \leq n+1$, are chosen such that $v\left(\sigma_{m}\right)$ is the grid point of $G_{m+1}$ nearest to the approximate fixed point $x^{*}$ in $\sigma_{m}$. Moreover, in practice we have that $k_{m}$ is not predetermined but that $\mathrm{k}_{\mathrm{m}}$ is chosen as soon as $\sigma_{\mathrm{m}}$ is generated. It will appear that the triangulation of $s^{n} \times\left[d_{m}, d_{m+1}\right]$ is determined by the numbers $\lambda_{1}^{m}, \ldots, \lambda_{n+1}^{m}$. Let $\tau\left(y^{1}, \pi\left(I_{n+1}\right)\right)$ be an arbitrarily chosen simplex of $G_{m}$ with $s\left(y^{1}\right)=1$, i.e. $\tau$ has the s-representation. To triangulate $s^{n} \times\left[d_{m}, d_{m+1}\right]$ we first triangulate $\tau\left(\mathrm{y}^{1}, \pi\left(I_{\mathrm{n}+1}\right)\right) \times\left[\mathrm{d}_{\mathrm{m}}, \mathrm{d}_{\mathrm{m}+1}\right]$ and then we prove that the union of the triangulations of $\tau \times\left[d_{m}, d_{m+1}\right]$ over all $n$-simplices $\tau$ of $G_{m}$ is a triangulation of $s^{n} \times\left[d_{m}, d_{m+1}\right]$. In the sequel we call the grid point $v(\tau)=\sum_{i=1}^{n+1} \mathrm{k}_{m}^{-1} \lambda_{i} \mathrm{~m}^{i}$ the centrepoint of the simplex $\tau\left(y^{1}, \pi\left(I_{n+1}\right)\right)$. The triangulation of $s^{n} \times\left[d_{m}, d_{m+1}\right]$ will be such that, for all $\tau$ of $G_{m}$, the $(n+1)$-simplex which is the convex hull of $\tau \times\left\{d_{m}\right\}$ and $v(\tau) \times\left\{d_{m+1}\right\}$ is an $(n+1)$-simplex of this triangulation. So, in particular we have that the $(n+1)-\operatorname{simplex} \psi_{m^{\prime}}$ being the convex hull of $\sigma_{m} \times\left\{d_{m}\right\}$ and $v\left(\sigma_{m}\right) \times\left\{d_{m+1}\right\}$ is a simplex of the triangulation.

To triangulate the set $\tau\left(y^{1}, \pi\left(I_{n+1}\right)\right) \times\left[d_{m}, d_{m+1}\right]$ we define for any proper subset $T$ of $I_{n+1}$ the regions $\AA^{\tau}(T)$ by

$$
\AA^{\tau}(T)=\left\{x \in \tau \mid x=v(\tau)+\sum_{j \in T} \mu_{j} q\left(\pi_{j}\right) \text { for positive numbers } \mu_{j}, j \in T\right\}
$$

Now a triangulation of $\tau \times\left[\mathrm{d}_{\mathrm{m}}, \mathrm{d}_{\mathrm{m}+1}\right]$ is obtained by connecting all the grid points $x$ of $G_{m+1}$ in $\AA \tau(T)$ on level $a_{m+1}$ with the vertices $y^{i}$, $i \notin T$, on level $d_{m}$. The proof that $\tau \times\left[a_{m}, d_{m+1}\right]$ is indeed triangulated is analogous to the proof of theorem 5.5.1. In fact, the triangulation of $\tau \times\left[d_{m}, d_{m+1}\right]$ is analogous to the triangulation of $s^{n} \times[0,1]$, as described in section 5.5 . In comparison with the definition of the regions $\AA(T)$ in section 5.3 , the regions 
${ }_{\AA} \tau(T)$ are adapted for the fact that for the simplex $\tau\left(y^{1}, \pi\left(I_{n+1}\right)\right), \pi\left(I_{n+1}\right)$ is an arbitrary permutation of the elements of $I_{n+1}$, whereas $s^{n}$ can be considered as the simplex $\sigma\left(e(1), \bar{\pi}\left(I_{n+1}\right)\right)$ of $G_{0}$ with $\bar{\pi}_{i}=i, i=1, \ldots, n+1$. For a simplex $\sigma\left(y^{1}, \pi\left(I_{n+1}\right)\right)$ with $\pi\left(I_{n+1}\right)=(1,3,2)$ the regions $A^{\tau}(T)$ (the closure of $\AA_{\AA}^{\tau}(T)$ in $\tau$ ) are given in figure 8.2.1.

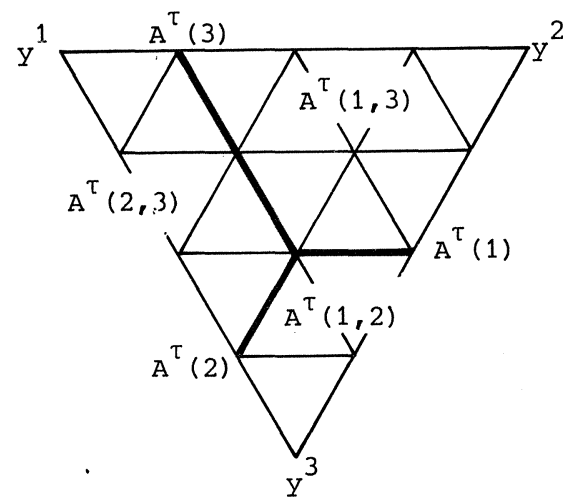

Figure 8.2.1. The regions $A^{\tau}(T)$ where $\tau\left(y^{1}, \pi\left(I_{n+1}\right)\right)$ is a simplex with $\pi\left(I_{n+1}\right)=(1,3,2)$.

Observe that $v(\tau) \times\left\{d_{m+1}\right\}$ is connected with all the vertices $y^{i}, i \in I_{n+1}$. Hence, $\operatorname{conv}\left(v(\tau) \times\left\{d_{m+1}\right\} \cup \tau \times\left\{d_{m}\right\}\right)$ is indeed a simplex of the triangulation. Moreover, we have that for $m=0 G_{0}$ consists of only one simplex,being $s^{n}$ itself. It can easily be seen that $s(e(i))=i, i \in I_{n+1}$. So it follows immediately that the triangulation of $s^{n} \times\left[a_{0}, a_{1}\right]$ is identical to the triangulation of $s^{\mathrm{n}} \times[0,1]$ (section 5.5 ).

THEOREM 8.2.2. The union of the triangulations of $\tau\left(y^{1}, \pi\left(I_{n+1}\right)\right) \times\left[d_{m}, d_{m+1}\right]$ over all n-simplices $\tau\left(y^{1}, \pi\left(I_{n+1}\right)\right)$ of $G_{m}$ triangulates $s^{n} \times\left[d_{m}, d_{m+1}\right]$.

PROOF. The triangulation of $\tau \times\left[d_{m}, d_{m+1}\right]$ is well-defined for any simplex $\tau$ of $G_{m}$. Let $\tau_{1}$ and $\tau_{2}$ be two adjacent $n$-simplices of $G_{m}$ and let $x$ be a grid point of $\mathrm{G}_{\mathrm{m}+1}$ in the common facet. Then it is sufficient to prove that, if in the triangulation of $\tau_{1} \times\left[d_{m} ; d_{m+1}\right], x$ is connected with a vertex $y$ of $\tau_{1} \cap \tau_{2}, x$ is also connected with $y$ in the triangulation of $\tau_{2} \times\left[d_{m}, d_{m+1}\right]$. 
Let $\tau_{1}$ be $\tau_{1}\left(v^{1}, \gamma\left(I_{n+1}\right)\right)$ and let $\tau_{2}$ be $\tau_{2}\left(w^{1}, \delta\left(I_{n+1}\right)\right)$. We have for both simplices the s-representation. Since $\tau_{1}$ and $\tau_{2}$ are adjacent, there exists a unique index $j$ such that $v^{i}=w^{i}, i \neq j$ and $\gamma_{i}=\delta_{i} i \neq j-1, j$ with the convention that $j-1=n+1$ if $j=1$. Moreover $\delta_{j-1}=\gamma_{j}$ and $\delta_{j}=\gamma_{j-1}$. So all the vertices $y$ in $\tau_{1} \cap \tau_{2}$ have the same index, $i$.e. if for some index $i \mathrm{y}=\mathrm{v}^{i}$ we have that $y=w^{i}$ for the same index $i$. Therefore we only have to prove that for any grid point $x$ of $G_{m+1}$ in $\tau_{1} \cap \tau,{ }_{2}, \AA^{\circ} \AA^{\tau}(T)$ implies that $x \in \AA^{\circ} 2(T)$. Since $x$ is a grid point of $G_{m+1}$ in the facet of $\tau_{1}$ opposite to $v^{j}$, there exist unique integers $\Theta_{i}, i \neq j-1, j$, such that

$$
x=v^{j+1}+\sum_{i \neq j-1, j} \Theta_{i} q\left(\gamma_{i}\right) / d_{m+1}
$$

with the convention that $v^{j+1}=v^{1}$ if $j=n+1$. Since $x \in \stackrel{\circ}{\AA}^{\tau} 1(T)$ for just one $T$, there exist also unique integers $\mu_{h}$ with $\mu_{h}>0, h \in T$ and $\mu_{h}=0, h \notin T$, such that

$$
x=v\left(\tau_{1}\right)+\sum_{h=1}^{n+1} \mu_{h} q\left(\gamma_{h}\right) / d_{m+1}
$$

or

$$
\begin{aligned}
& x=\sum_{h=1}^{n+1} \lambda_{h}^{m} v^{h} / k_{m}+\mu_{j-1} q\left(\gamma_{j-1}\right) / d_{m+1}+\mu_{j} q\left(\gamma_{j}\right) / d_{m+1}+ \\
& +\sum_{h \neq j-1, j} \mu_{h} q\left(\gamma_{h}\right) / d_{m+1} \\
& =\sum_{h=1}^{j} \lambda_{h}^{m}\left\{v^{j+1}+\sum_{i=j+1}^{n+1} q\left(\gamma_{i}\right) / d_{m}+\sum_{i=1}^{h-1} q\left(\gamma_{i}\right) / d_{m}\right\} / k_{m}+ \\
& +\sum_{h=j+1}^{n+1} \lambda_{h}^{m_{\{v} j+1}+\sum_{i=j+1}^{h-1} q\left(\gamma_{i}\right) / d_{m}^{-\} / k_{m}}+\mu_{j-1} q\left(\gamma_{j-1}\right) / d_{m+1}+ \\
& +\mu_{j} q\left(\gamma_{j}\right) / d_{m+1}+\sum_{h \neq j-1, j} \mu_{h} q\left(\gamma_{h}\right) / d_{m+1} \\
& =v^{j+1}+\left(\sum_{h=1}^{j} \lambda_{h}^{m}\right)\left(\sum_{i=j+1}^{n+1} q\left(\gamma_{i}\right) / d_{m+1}\right)+\sum_{h=1}^{j-1} \sum_{i=1}^{h-1} \lambda_{h}^{m} q\left(\gamma_{i}\right) / d_{m+1}+ \\
& +\sum_{i=1}^{j-2} \lambda_{j}^{m} q\left(\gamma_{i}\right) / d_{m+1}+\lambda_{j}^{m} q\left(\gamma_{j-1}\right) / d_{m+1}+\sum_{h=j+1}^{n+1} \sum_{i=j+1}^{h-1} \lambda_{h}^{m} q\left(\gamma_{i}\right) / d_{m+1}+ \\
& +\mu_{j-1} q\left(\gamma_{j-1}\right) / d_{m+1}+\mu_{j}\left(-\sum_{i \neq j} q\left(\gamma_{i}\right)\right) / d_{m+1}+\sum_{h \neq j-1, j} \mu_{h} q\left(\gamma_{h}\right) / d_{m+1} \cdot
\end{aligned}
$$


Defining $\theta_{i}^{\prime}$ to be the coefficient of $q\left(\gamma_{i}\right), i \neq j-1, j$, we obtain that (8.2.3) $\quad x=v^{j+1}+\sum_{i \neq j-1, j} \theta_{i}^{\prime} q\left(\gamma_{i}\right) / d_{m+1}+\left(\lambda_{j}^{m}+\mu_{j-1}-\mu_{j}\right) q\left(\gamma_{j-1}\right) / d_{m+1}$. Comparing (8.2.3) with $(8.2 .1)$ we obtain that $\Theta_{i}^{\prime}=\Theta_{i}, i \neq j-1, j$ and (8.2.4) $\quad \lambda_{j}^{m}+\mu_{j-1}-\mu_{j}=0$.

Moreover from (8.2.2) it follows that

$$
\begin{gathered}
x=\sum_{h \neq j} \lambda_{h}^{m} v^{h} / k_{m}+\lambda_{j}^{m}\left(v^{j-1}+q\left(\gamma_{j-1}\right) / d_{m}\right) / k_{m}+\mu_{j-1} q\left(\gamma_{j-1}\right) / d_{m+1}+ \\
+\mu_{j} q\left(\gamma_{j}\right) / d_{m+1}+\sum_{h \neq j-1, j} \mu_{h} q\left(\gamma_{h}\right) / d_{m+1} .
\end{gathered}
$$

Hence, since $v^{i}=w^{i}, i \neq j, \gamma_{i}=\delta_{i^{\prime}} i \neq j-1, j, \gamma_{j-1}=\delta_{j}$ and $\gamma_{j}=\delta_{j-1}$, we obtain that

$$
\begin{aligned}
x=\sum_{h \neq j} & \lambda_{h}^{m} w^{h} / k_{m}+\lambda_{j}^{m}\left(w^{j-1}+q\left(\delta_{j}\right) / d_{m}\right) / k_{m}+\mu_{j-1} q\left(\delta_{j}\right) / d_{m+1}+ \\
& +\mu_{j} q\left(\delta_{j-1}\right) / d_{m+1}+\sum_{h \neq j-1, j} \mu_{h} q\left(\delta_{h}\right) / d_{m+1}+\lambda_{j}^{m} q\left(\delta_{j-1}\right) / d_{m+1}- \\
& -\lambda_{j}^{m} q\left(\delta_{j-1}\right) / d_{m+1} .
\end{aligned}
$$

From (8.2.4) it follows that $\mu_{j}-\lambda_{j}^{m}=\mu_{j-1}$ and $\mu_{j-1}+\lambda_{j}^{m}=\mu_{j}$. Consequently,

$$
\mathrm{x}=\mathrm{v}\left(\tau_{2}\right)+\sum_{\mathrm{h}=1}^{\mathrm{n}+1} \mu_{\mathrm{h}} \mathrm{q}\left(\delta_{\mathrm{h}}\right) / \mathrm{d}_{\mathrm{m}+1}
$$

with $\mu_{h}$ the same coefficients as in $(8.2 .2)$. Hence $x \in \AA^{\tau} 2(T)$ which proves the theorem.

From the proof of theorem 8.2.2 we obtain the following corollary.

COROLLARY 8.2.3. If a grid point $x$ of $\mathrm{G}_{\mathrm{m}+1}$ belongs to two adjacent simplices $\tau_{1}\left(v^{1}, \gamma\left(I_{n+1}\right)\right)$ and $\tau_{2}\left(w^{1}, \delta\left(I_{n+1}\right)\right)$ of $G_{m}$ and if, for some $T$,

$$
\mathrm{x}=\mathrm{v}\left(\tau_{1}\right)+\sum_{h \in T} \mu_{h} \mathrm{q}\left(\gamma_{\mathrm{h}}\right) / \mathrm{d}_{\mathrm{m}+1}
$$


then, for the same coefficients $\mu_{h}$ '

$$
\mathrm{x}=\mathrm{v}\left(\tau_{2}\right)+\sum_{h \in T} \mu_{\mathrm{h}} \mathrm{q}\left(\delta_{\mathrm{h}}\right) / \mathrm{d}_{\mathrm{m}+1}
$$

This important fact will be frequently used in the replacement steps of the algorithm.

Theorem 8.2.2 is illustrated in figure 8.2.2 for $n=2, a_{1}=4, d_{2}=16$ and $\lambda^{1}=(2,1,1)$. So the grid size of the triangulation on level 1 is $1 / 4$ and the grid size of the triangulation on level $d_{2}$ is $1 / 16$. Let $\sigma_{1}\left(u^{1}, B\left(I_{n+1}\right)\right)$ with $u^{1}=\left(\frac{1}{2}, \frac{1}{4}, \frac{1}{4}\right)^{\top}$ and $B\left(I_{n+1}\right)=(1,3,2)$ be the starting simplex on level $d_{1}$. Then the centrepoint $u$ of $\sigma_{1}$ is $v\left(\sigma_{1}\right)=\sum_{i=1}^{n+1} \lambda_{i}^{1} u^{i}=(7 / 16,3 / 8,3 / 16)^{\top}$. For the arbitrarily chosen simplex $\tau\left(y^{1}, \pi\left(I_{n+1}\right)\right)$ of $G_{1}$ with $y^{1}=\left(0, \frac{1}{2}, \frac{1}{2}\right)^{\top}$ and $\pi\left(I_{n+1}\right)=(2,3,1)$ we have that the centrepoint $v$ is $v(\tau)=(1 / 16,3 / 8,9 / 16)^{\top}$. In the figure the triangulation $G_{2}$ on level $d_{2}$ is pictured. Furthermore, the vertices of $G_{1}$ are drawn heavily. For any simplex $\tau$ of $G_{1}$ the regions $A^{\tau}(T)$ are indicated by the heavy lines. Observe that for any two simplices $\tau_{1}$ and $\tau_{2}$ of $G_{1}$ we have that

$$
\left(\tau_{1} \cap \tau_{2}\right) \cap A^{\tau} 1(T)=\left(\tau_{1} \cap \tau_{2}\right) \cap A^{\tau} 2(T) .
$$

As is illustrated for the simplices $\tau$ and $\rho$ with $\tau$ as the simplex defined above and $\rho=\rho\left(\bar{y}^{-1}, \bar{\pi}\left(I_{n+1}\right)\right.$ with $\bar{y}^{-1}=\left(\frac{1}{4}, 0, \frac{3}{4}\right)$ and $\bar{\pi}\left(I_{n+1}\right)=(1,3,2)$, the region $A^{\tau}(1,2)$ adjoins in a consistent way to the region ${ }^{\circ} \rho(1,2)$. The grid points of $A^{\tau}(1,2)$ and $A^{\rho}(1,2)$ on level $d_{2}$ are connected with the heavily drawn vertex $\left(\frac{1}{4}, \frac{1}{4}, \frac{1}{2}\right)^{\top}$ on level $d_{1}$. 


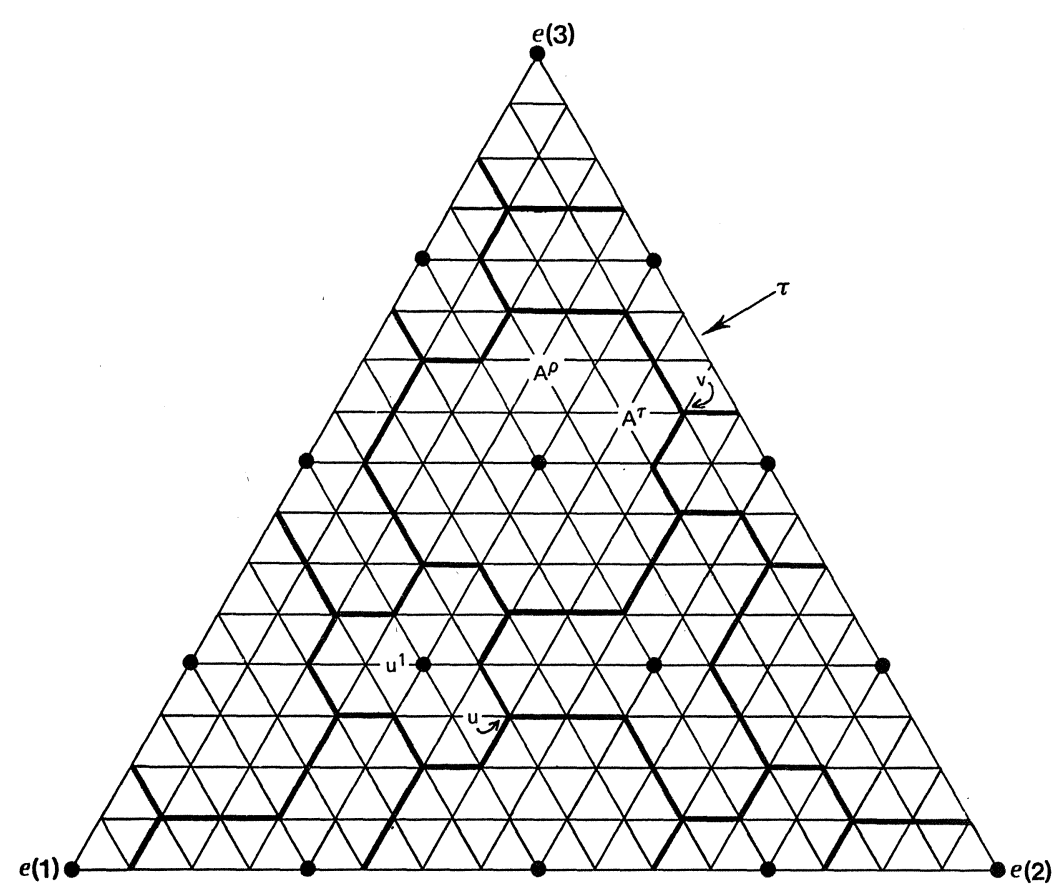

Figure 8.2.2. $n=2, a_{2}=16, \lambda^{1}=(2,1,1), \sigma=\sigma_{1}\left(u^{1}, B\left(I_{n+1}\right)\right)$ with $u^{1}=\left(\frac{1}{2}, \frac{1}{4}, \frac{1}{4}\right)^{\top}$ and $B\left(I_{n+1}\right)=(1,3,2), u=v\left(\sigma_{1}\right), v=v(\tau)$. where $\tau=\tau\left(y^{1}, \pi\left(I_{n+1}\right)\right)$ with $\mathrm{y}^{1}=\left(0, \frac{1}{2}, \frac{1}{2}\right){ }^{\top}$ and $\pi\left(I_{n+1}\right)=(2,3,1) ; A^{\tau}=A^{\tau}(1,2)$ and $A^{\rho}=A^{\rho}(1,2)$. The vertices of $G_{1}$ are drawn heavily. The grid points of $G_{2}$ on level $d_{2}$ within or on the boundary of a region surrounded by heavy lines are connected with the grid point of $G_{1}$ in the middle of it on level $d_{1}$.

\subsection{THE ALGORITHM.}

Suppose that we want to compute a fixed point of a continuous function $f$ from $S^{n}$ into itself by applying the continuous deformation algorithm with a triangulation of $s^{n} \times[1, \infty)$ as described in the previous section. Using integer labelling, each point $\left(x^{\top}, d_{i}\right)^{\top}$ of $s^{n} \times\left\{d_{i}\right\}, i=0,1, \ldots$, receives the standard integer label $\ell(x)$ as defined in definition 4.1.1. 
For some arbitrarily chosen grid point $v$ of $G_{1}$, e.g. $v$ is the grid point nearest to the barycenter $(1 / n+1, \ldots, 1 / n+1)^{\top}, s^{n} \times\left[d_{0}, d_{1}\right]$ is triangulated as described in the previous section with $\sigma_{0}=S^{n}$ and $v=v\left(\sigma_{0}\right)=\sum_{i=1}^{n+1} \lambda_{i}^{0} e(i)$ the centrepoint of $\sigma_{0}$. The algorithm starts with the simplex $\psi_{0}$, being the convex hull of the vertices of $s^{n}$ on level $d_{0}$ and the point $v$ on level $d_{1}$, and proceeds along a path of adjacent $(n+1)$-simplices in $s^{n} \times\left[d_{0}, d_{1}\right]$ such that the common facets are completely labelled. Note that $s^{n} \times\left\{d_{0}\right\}$ is a completely labelled facet of $\psi_{0}$. Clearly, since the labelling rule is proper, there are no completely labelled facets in (bd $\left.s^{n}\right) \times\left[d_{0}, d_{1}\right]$. Therefore all replacement steps are feasible and the algorithm generates within a finite number of steps a simplex in $S^{n} \times\left[d_{0}, d_{1}\right]$ having a completely labelled facet in $s^{n} \times\left\{d_{1}\right\}$, say $\sigma_{1}\left(u^{1}, \beta\left(I_{n+1}\right)\right)$. Note that the intersection of the path of adjacent simplices with $s^{n} \times\left\{d_{1}\right\}$ is the path of adjacent $t$-simplices $(0 \leq t \leq n)$ of $G_{1}$ generated by the variable dimension restart algorithm as described in chapter 5 . Now an integer $k_{1} \geq 2$ is chosen and $s^{n_{1}} \times\left[d_{1}, d_{2}\right]$ is triangulated as described in the previous section with $\sigma_{1}$ as the starting simplex and an arbitrary grid point $u=\sum_{i=1}^{n+1} \lambda_{i}^{1} u^{i} / k_{1}$ of $G_{2}$ in $\sigma_{1}$ as the centre point $v\left(\sigma_{1}\right)$ of $\sigma_{1}$. Then the algorithm continues the path of adjacent simplices with completely labelled common facets by replacing the vertex of $s^{n} \times\left\{d_{0}\right\}$ connected with the vertices of $\sigma_{1} \times\left\{d_{1}\right\}$ by $v\left(\sigma_{1}\right) \times\left\{d_{2}\right\}$ and computing $\ell\left(v\left(\sigma_{1}\right)\right)$. Simplices in $s^{n} \times\left[d_{1}, d_{2}\right]$ are generated until a simplex is found having either a completely labelled facet, say $\tau_{1}$, in $s^{n} \times\left\{d_{1}\right\}$ or a completely labelled facet in $s^{\mathrm{n}} \times\left\{\mathrm{d}_{2}\right\}$. In the latter case $\mathrm{k}_{2}$ and $\lambda_{1}^{2}, \ldots, \lambda_{n+1}^{2}$ are chosen and the algorithm continues with simplices in $s^{n} \times\left[d_{2}, d_{3}\right]$. In the first case the vertex $v\left(\tau_{1}\right) \times\left\{d_{2}\right\}$, which is connected with the vertices of $\tau_{1}$, is replaced by the vertex of $s^{n}$ on level $d_{0}$ connected with the vertices of $\tau_{1}$, and the algorithm proceeds with a path of adjacent simplices of the triangulation of $\mathrm{s}^{\mathrm{n}} \times\left[\mathrm{d}_{0_{1}} \mathrm{~d}_{1}\right]$, until again a simplex having a completely labelled facet, say $\tau_{2}\left(y^{1}, \pi\left(I_{n+1}\right)\right)$, in $s^{n} \times\left\{d_{1}\right\}$ is generated. Then the algorithm continues with simplices in $S^{n} \times\left[d_{1}, d_{2}\right]$ by replacing the vertex in $S^{n} \times\left\{d_{0}\right\}$ by $v\left(\tau_{2}\right) \times\left\{d_{2}\right\}$, where $v\left(\tau_{2}\right)=\sum_{i=1}^{n+1} \lambda_{i}^{1} y^{i} / k_{1}$ is the centrepoint of $\tau_{2}$.

within a finite number of steps the algorithm generates a simplex having a completely labelled facet in $s^{n} \times\left\{d_{2}\right\}$, since the replacement steps are unique and feasible and the number of simplices of the triangulation of $s^{n} \times\left[d_{0}, d_{2}\right]$ is finite. Then $k_{2}$ and $\lambda_{i}^{2}, i \in I_{n+1}$, are chosen and the algorithm proceeds along a path of simplices in $s^{n} \times\left[d_{0}, d_{3}\right]$ etc. The algorithm can be 
terminated if it generates a simplex of $G_{m}$ with grid size $d_{m}^{-1}$ small enough. Clearly, within a finite number of steps the algorithm finds a completely labelled simplex of $\mathrm{G}_{\mathrm{m}}$. Using vector labelling a vertex $e(h)$ of $s^{n}$ on level $d^{0}$ is labelled by $e(h), h=1, \ldots, n+1$, and a point $x$ on level $d_{i^{\prime}} i \geq 1$, is labelled by $l(x)=$ $x-f(x)+e$. The algorithm starts with the same simplex $\psi_{0}$ by introducing the vector $\ell\left(v\left(\sigma_{0}\right)\right)$ in the system of $n+1$ linear equations $I \mu=e$. To compute a fixed point of a mapping $\phi$, a point $x$ on level $d_{i^{\prime}} i \geq 1$, is labelled by $\ell(x)=x-f^{i}(x)+e$, where $f^{i}(x)$ is a piecewise linear approximation to $\phi$ with respect to $G_{i}$. The algorithm can be seen as tracing a path of zeroes of the piecewise linear function $f(x, t)$ obtained by taking the functions $\bar{\phi}\left(x, a_{i}\right), i \geq 1$, defined by

$$
\bar{\phi}(x, 1)=(n+1) x-e \text { and } \bar{\phi}\left(x, a_{i}\right)=x-f^{i}(x) \quad i \geq 1
$$

and extending them linearly on each simplex of the triangulation of $s^{\mathrm{n}} \times[1, \infty)$.

\subsection{THE REPLACEMENT STEPS.}

To give the replacement steps it is more appropriate to describe the triangulation of the set $\tau\left(y^{1}, \pi\left(I_{n+1}\right)\right) \times\left[d_{m}, d_{m+1}\right]$ as follows. For any subset $T$ of $I_{n+1}$, let the regions $\AA^{\tau}(T)$ be redefined by

$$
\AA_{\AA}^{\tau}(T)=\left\{x \in \tau \mid x=v(\tau)+\sum_{j \in T} \mu_{j} q(j) \text { for positive numbers } \mu_{j^{\prime}} j \in T\right\} \text {. }
$$

Then $\tau \times\left[d_{m}, d_{m+1}\right]$ is triangulated by connecting all the grid points $x$ of $G_{m+1}$ in $\AA_{A}^{\tau}(T)$ on level $d_{m+1}$ with every vertex $y^{i}$ of $\tau$ on level $d_{m}$, such that $\pi_{i} \notin T$. It can easily be verified that this triangulation of $\tau \times\left[d_{m}, d_{m+1}\right]$ is identical to the triangulation given in section 2 . However we remark the following. In section 2 a grid point $x$ in $\AA^{\circ} \tau(T)$ on level $d_{m+1}$ is connected with the vertex $y^{i}$ of $\tau$ on level $d_{m}$ if $i \notin T$. By this definition $x \in \tau_{1} \cap \tau_{2}$ and $x \in \AA^{\tau} 1$ 8.2.2. Now we have that $x$ in $\AA^{\tau}(T)$ is connected with $y^{i}$ if $\pi_{i} \notin T$. So, for some $s$, let $y^{j}, j \neq s$, be the common vertices of $\tau_{1}$ and $\tau_{2}$ and let $T^{1}$ and $T^{2}$ be the subsets of $I_{n+1}$ such that $x \in \AA^{\tau} i\left(T^{i}\right), i=1,2$. Then with the convention that $s-1=n+1$ if $s=1$, 


$$
\begin{aligned}
& T^{2}=T^{1} \text { if } \pi_{s-1}, \pi_{s} \notin T^{1} \text { or } \pi_{s-1}, \pi_{s} \in T^{1} \\
& T^{2}=\left(T^{1} \cup\left\{\pi_{s}\right\}\right) \backslash\left\{\pi_{s-1}\right\} \text { if } \pi_{s-1} \in T^{1} \text { and } \pi_{s} \notin T^{1} \\
& T^{2}=\left(T^{1} \cup\left\{\pi_{s-1}\right\}\right) \backslash\left\{\pi_{s}\right\} \text { if } \pi_{s-1} \notin T^{1} \text { and } \pi_{s} \in T^{1} .
\end{aligned}
$$

Moreover, let $\mu_{1}, \ldots, \mu_{n+1}$ with $\mu_{h}>0$ if $h \in T^{1}$ and $\mu_{h}=0$ if $h \notin T^{1}$ be the numbers such that

$$
\mathrm{x}=\mathrm{v}\left(\tau_{1}\right)+\sum_{h=1}^{\mathrm{n}+1} \mu_{\mathrm{h}} \mathrm{q}(\mathrm{h}) / d_{\mathrm{m}+1}
$$

Then, by corollary 8.2.3,

$$
\mathrm{x}=\mathrm{v}\left(\tau_{2}\right)+\sum_{\mathrm{h}=1}^{\mathrm{n}+1} \mu_{\mathrm{h}}^{\prime} \mathrm{q}(\mathrm{h}) / \mathrm{d}_{\mathrm{m}+1}
$$

with $\mu_{h}^{\prime}=\mu_{h}, h \neq s-1, s, \mu_{s-1}^{\prime}=\mu_{s}$ and $\mu_{s}^{\prime}=\mu_{s-1}$. Now for some $m$, let $\psi$ be a simplex in $s^{n} \times\left[d_{m}, d_{m+1}\right]$ generated by the algorithm. Since $\psi$ is a simplex of the triangulation of $s^{n} \times\left[d_{m}, d_{m+1}\right]$, there exist a simplex $\tau\left(v^{1}, \gamma\left(I_{n+1}\right)\right)$ of $s^{n} \times\left\{d_{m}\right\}$ with $v(\tau)=\sum_{i=1}^{n+1} \lambda_{i}^{m} v^{i}$ as its centrepoint, a subset $T$ of $t$ elements of $I_{n+1}$, a permutation $\pi(T)$ and a nonnegative vector $\left(R_{1}, \ldots, R_{n+1}\right)^{\top}$ of integers such that the following conditions hold:

1. The intersection of $\psi$ and $s^{n} \times\left\{d_{m}\right\}$ is the convex hull of the $n+1-t$ vertices $v^{i}$ of $\tau$ such that $\gamma_{i} \notin T$. This set of vertices is called the set of active vertices of $\tau$, whereas the other vertices are called inactive,

2. The intersection of $\psi$ and $s^{n} \times\left\{d_{m+1}\right\}$ is the t-simplex $\sigma\left(y^{1}, \pi(T)\right)$ with vertices $y^{1}, \ldots \ldots, y^{t+1}$ in $\tau \times\left\{d_{m+1}\right\}$ such that
a) $y^{1}=v(\tau)+\sum_{j=1}^{n+1} R_{j} q(j) / d_{m+1}$
b) $y^{i+1}=y^{i}+q\left(\pi_{i}\right) / d_{m+1} \quad i=1, \ldots, t$,

3. $R_{j}=0$ for $j \notin T$ and $R_{j} \geq 0$ for $j \in T$.

Observe that the conditions $2 a, b$ and 3 are analogous to respectively a,c and $b$ on page 66 . In particular the conditions $1-3$ are satisfied for the starting simplex of the algorithm given by the convex hull of $s^{n} \times\left\{d_{0}\right\}$ and $v\left(\sigma_{0}\right) \times\left\{d_{1}\right\}$. So the algorithm is initiated with $\tau\left(v^{1}, \gamma\left(I_{n+1}\right)\right)=\sigma_{0}\left(e(i), \tilde{\gamma}\left(I_{n+1}\right)\right)$ 
with $\tilde{\gamma}_{i}=i, i \in I_{n+1}, T=\varnothing, \pi(T)=(\varnothing)$ and $R_{i}=0, i \in I_{n+1}$.

Now any replacement step can be described by adapting $\tau\left(v^{1}, \gamma\left(I_{n+1}\right)\right)$, the subset $T$, the permutation $\pi(T)$ and the vector $R$. Recall that $\tau$ has always the s-representation.

As described in the previous section two facets of $\psi$ are completely labelled. So, only two vertices of $\psi$ have the same label and one of them is the last vertex generated by the algorithm. Then the other one must be replaced. Two cases can occur,

A) the vertex to be replaced is an active vertex of $\tau\left(v^{1}, \gamma\left(I_{n+1}\right)\right)$, say $v^{i_{0}}$,

B) the vertex to be replaced is a vertex of $\sigma\left(y^{1}, \pi(T)\right)$, say $y^{j_{0}}$.

We consider now these two cases.

${ }_{1}^{\mathrm{e}}$. Suppose that $\mathrm{v}^{i_{0}}$ is the only active vertex of $\tau\left(v^{1}, \gamma\left(I_{n+1}\right)\right)$. Then $|T|=n$ and $\sigma\left(y^{1}, \pi(T)\right)$ is a completely labelled $n$-simplex of $s^{n}$ on level $d_{m+1}$. Now the simplex $\sigma$ is written in the s-representation and $\tau$ is set equal to $\sigma, R$ is set equal to zero and $T$ becomes the empty set. The ' algorithm continues by computing the label of the centrepoint $v(\tau)=\Sigma_{i=1}^{n+1} \lambda_{i}^{m+1} v^{i}$ of the new simplex $\tau$. If it is the first time that a completely labelled simplex is found on level $d_{m+1^{\prime}}$ this simplex is the starting simplex $\sigma_{m+1}$ on level $d_{m+1}$ and the integers $\lambda_{i}^{m+1}, i=1, \ldots, n+1$, can be chosen arbitrarily.

$2^{e}$. The vertex $v^{i_{0}}$ is not the only active vertex of $\tau\left(v^{1}, \gamma\left(I_{n+1}\right)\right)$.

First suppose that

$$
\lambda_{i_{0}}^{m} \neq 0 \text { or } \gamma_{i_{0}-1} \in T \text { (with } i_{0}-1=n+1 \text { if } i_{0}=1 \text { ). }
$$

Since $\mathrm{v}^{i_{0}}$ is active we have that $\gamma_{i_{0}} \notin T$ and therefore $\mathrm{y}^{t+1}$ is not on the facet of $\tau$ opposite to $v^{i_{0}}$. Hence the point $y^{t+2}=y{ }^{t+1}+q\left(\gamma_{i_{0}}\right) / d_{m+1}$ is a grid point of $\mathrm{G}_{\mathrm{m}+1}$ in $\tau$. Now $\mathrm{v}^{i_{0}}$ becomes an inactive vertex of $\tau$ and is replaced by $y^{t+2}$, i.e. $T$ is set equal to $T u\left\{\gamma_{i_{0}}\right\}$ and $\pi(T)$ becomes $\left(\pi_{1}, \ldots, \pi_{t}, \gamma_{\dot{L}_{0}}\right)$, whereas the simplex $\tau$ and the vector $R$ do not change. If both 


$$
\lambda_{i_{0}}^{m}=0 \text { and } \gamma_{i_{0}-1} \notin T
$$

then $y^{1}, \ldots, y^{t+1}$ are points on the facet of $\tau$ opposite to $v^{i_{0}}$ and the point $y^{t+1}+q\left(\gamma_{i_{0}}\right) / d_{m+1}$ is a grid point of $G_{m+1}$ not in $\tau$. Now $\tau$ is adapted according to table 8.2 .1 by replacing $v^{i} 0$. Let $\tau_{1}$ be the new simplex on level $d_{m}$ and let $\bar{v}^{i} 0$ be the new vertex of $\tau_{1}$. Since $y^{1}, \ldots, y^{t+1}$ are on the common facet of $\tau$ and $\tau_{1}$, it follows from the proof of theorem 8.2.2 that again a simplex of the triangulation is obtained if $v{ }^{i} 0_{x}\left\{d_{m}\right.$ is replaced by $\vec{v}^{i} 0_{x}\left\{d_{m}\right\}$. By corollary 8.2 .3 and the fact that $\gamma_{i_{0}}$ and $\gamma_{i_{0}}-1$ are both not in $T$ it follows that $T, \pi(T)$ and $R$ do not change. Hence $\tau$ becomes $\tau_{1}$ and $\bar{v}^{i} 0_{\times}\left\{d_{m}\right\}$ is the new vertex.

Case B.

Suppose that $t>0$. Then we consider the following cases,

$$
\begin{array}{ll}
\text { (i) } & j_{0}=1 \\
\text { (ii) } & 2 \leq j_{0} \leq t \\
\text { (iii) } & j_{0}=t+1
\end{array}
$$

In case (i) we first suppose that

$$
\gamma_{r-1} \in T \text { (with } r-1=n+1 \text { if } r=1 \text { ) or } R_{\gamma_{r}}+1<\lambda_{r}^{m} \text {, }
$$

where $r$ is the index such that $\pi_{1}=\gamma_{r}$. Then $y^{t+1}$ is not on the facet of $\tau$ opposite $v^{r}$ and therefore $\mathrm{y}^{\mathrm{t}+1}+\mathrm{q}\left(\pi_{1}\right) / \mathrm{d}_{\mathrm{m}+1}$ is a grid point of $\mathrm{G}_{\mathrm{m}+1}$ in $A^{\tau}(T)$. Now $\pi(T)$ and $R$ are adapted according to table 5.2.1, i.e. $y^{1}$ is replaced by $y^{t+1}+q\left(\pi_{1}\right) / a_{m+1}$.

If both

$$
\gamma_{r-1} \notin T \text { and } R_{\gamma_{r}}+1=\lambda{ }_{r^{\prime}}^{m}
$$

then $y^{2}, \ldots, y^{t+1}$ are on the facet of $\tau$ opposite $v^{r}$ and $y^{t+1}+q\left(\pi_{1}\right) / d_{m}$ is not in $\tau$. Let $\tau\left(\bar{v}^{1}, \bar{\gamma}\left(I_{n+1}\right)\right)$ be the simplex obtained from $\tau\left(v^{1}, \gamma\left(I_{n+1}\right)\right)$ by replacing the inactive vertex $\mathrm{v}^{r}\left(\gamma_{r}=\pi_{1} \in T\right)$ according to table 8.2.1. Now $T$ becomes $\left(T \cup\left\{\gamma_{r-1}\right\}\right) \backslash\left\{\gamma_{r}\right\}, R_{\gamma_{r}}$ and $R_{\gamma_{r-1}}$ are interchanged, $\pi_{1}$ becomes $\gamma_{r-1}$ and $\tau$ is set equal to $\left.\tau\left(\bar{v}^{-1}, \bar{r}_{\left(I_{n+1}\right.}\right)\right)$, implying that $y^{1}$ is replaced by the new 
vertex $y^{1}$, defined by $v(\tau)+\sum_{j=1}^{n+1} R_{j} q(j)$ for the new $\tau$ and $R$. In case (ii) suppose that

$$
\pi_{j_{0}-1} \neq \gamma_{r-1} \text { (with } r-1=n+1 \text { if } r=1 \text { ) or } R_{\gamma_{r}}{ }^{-R_{\gamma_{r-1}}}<\lambda_{r}^{m} \text {, }
$$

where $r$ is the index such that $\pi_{j_{0}}=\gamma_{r}$. Then $\pi(T)$ and $R$ are adapted according to table 5.2.1,i.e. $y^{j_{0}}$ is replaced by $y^{j_{0}-1}+q\left(\pi j_{0}\right) / d_{m+1}$ which is

If both

$$
\pi_{j_{0}-1}=\gamma_{r-1} \text { and } R_{\gamma_{r}}-R_{\gamma_{r-1}}=\lambda_{r}^{m}
$$

then $y^{i}, i \neq j_{0^{\prime}}$ is on the facet of $\tau$ opposite $v^{r}$ and $y^{j_{0}-1}+q\left(\pi_{j_{0}}\right) / d_{m+1}$ is not in $\tau$. Now $\pi(T)$ is adapted according to table 5.2.1, $R_{\gamma_{r}}$ and $R_{\gamma_{r-1}}$ are interchanged and $\tau\left(v^{1}, \gamma\left(I_{n+1}\right)\right)$ is adapted according to table 8.2 .1 by replacing the inactive vertex $v^{r}, i . e . y^{j}$ is replaced by the new vertex $\bar{y}^{j}$ defined by the new $\tau, R$ and $\pi(T)$. Observe that $T$ does not change since $\gamma_{r}$ and $\gamma_{r-1}$ are both an element of $T$.

In case (iii) suppose that $R_{\pi_{t}} \geq 1$. Then $y^{t+1}$ is replaced by the grid point $y^{1}-q\left(\pi_{t}\right) / d_{m+1}$ in $A^{\tau}(T)$, i.e. $\pi(T)$ and $R$ are adapted according to table 5.2.1. If $R_{\pi_{t}}=0, y^{1}-q\left(\pi_{t}\right) / d_{m+1}$ is not in $A^{\tau}(T)$. Now $y^{t+1}$ must be replaced by the inactive vertex $v^{r}$ of $\tau\left(v^{1}, \gamma\left(I_{n+1}\right)\right)$, where $r$ is the index with $\gamma_{r}=\pi_{t}$. So $\pi(T)$ becomes $\left(\pi_{1}, \ldots, \pi_{t-1}\right)$ and $T$ becomes $T \backslash\left\{\pi_{t}\right\}$.

Finally we consider the case $t=0$. Then $T=\varnothing$ and the only vertex $y^{1}$ of the zero-dimensional simplex $\sigma\left(y^{1}, \pi(T)\right)$ has to be replaced. Since $T=\phi, R_{j}=0$ $j \in I_{n+1}$ and $y^{1}$ is the centrepoint $v(\tau)$ of $\tau\left(v^{1}, \gamma\left(I_{n+1}\right)\right)$, which is connected with all vertices of $\tau$. So, all these vertices are active and $\tau$ is a completely labelled simplex of $\mathrm{G}_{\mathrm{m}}$. Hence $\mathrm{y}^{1}$ has to be replaced by a grid point of $G_{m-1}$ on level $d_{m-1}$. Therefore we have to compute both the simplex $\bar{\rho}\left(\bar{z}_{1}, \bar{B}\left(I_{n+1}\right)\right)$ of $s^{n} \times\left\{d_{m-1}\right\}$ such that all vertices of $\tau$ are grid points of $G_{m}$ in $\bar{\rho}$, and the vertex of $\bar{\rho}$, say $\bar{z}^{i} 0$, which is connected with all the vertices of $\tau$. To do so, we choose an arbitrary interior point of $\tau$, say $x$, and we calculate the vector $\alpha(x)$ as described in section 2 with $\mathrm{d}_{i}=\mathrm{d}_{\mathrm{m}-1}$. Since $\mathrm{x}$ is not a grid point of $\mathrm{G}_{\mathrm{m}-1}, \alpha(\mathrm{x})$ is not a vector of integers. Let $\bar{\alpha}_{i}$ be the entier of $\alpha_{i}(x), i=1, \ldots, n$, and let $z^{1}$ be the grid 
point of $G_{m-1}$ such that $z^{1}=e(1)+\sum_{i=1}^{n} \bar{\alpha}_{i} q(i) / d_{m-1}$. Since $x$ is an interior point of $\tau, \alpha_{i}(x)-\bar{\alpha}_{i} \neq \alpha_{j}(x)-\bar{\alpha}_{j}$ for all $i \neq j, j=1, \ldots, n$. Let $\beta\left(I_{n+1}\right)$ be the permutation of the elements of $I_{n+1}$ such that

$$
\alpha_{\beta_{1}}(x)-\bar{\alpha}_{\beta_{1}}>\alpha_{\beta_{2}}(x)-\bar{\alpha}_{\beta_{2}}>\ldots>\alpha_{\beta_{n}}(x)-\bar{\alpha}_{\beta_{n}}
$$

and $\beta_{n+1}=n+1$. Then $x$ is an interior point of the simplex $\rho\left(z^{1}, \beta\left(I_{n+1}\right)\right)$ of

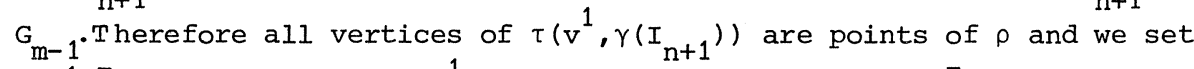
$\bar{\rho}\left(\bar{z}^{1}, \bar{\beta}\left(I_{n+1}\right)\right)$ equal to $\rho\left(z^{1}, \beta\left(I_{n+1}\right)\right)$ in such a way that $\bar{\rho}$ has the s-representation. It remains to find $\bar{z}^{i}$. Let $v(\bar{\rho})=\sum_{i=1}^{n+1} \lambda_{i}^{m-1} z^{i} / d_{m}$ be the centrepoint of $\bar{\rho}$. It can be easily seen that

$$
v^{1}=v(\bar{\rho})+\sum_{j=1}^{n} \theta_{j} q(j) / d_{m}
$$

with $\theta_{j}=\sum_{i=1}^{j}\left(v_{i}(\bar{\rho})-v_{i}^{1}\right), j=1, \ldots, n$. Set $\theta_{n+1}$ equal to zero and define (8.4.1) $\bar{\Theta}_{j}=\Theta_{j}-\min _{i \in I_{n+1}} \theta_{i} \quad j=1, \ldots, n+1$.

Since $\sum_{i=1}^{n+1} q(i)=0$, we obtain that $v^{1}=v(\bar{\rho})+\sum_{j=1}^{n+1} \bar{\theta}_{j} q(j) / d_{m}$. Define for $i=1, \ldots, n+1, \theta^{i}=\left(\theta_{1}^{i}, \ldots, \theta_{n+1}^{i}\right)$ by

(8.4.2) $\begin{cases}\Theta_{j}^{i}=\bar{\theta}_{j}+1 & \text { if there exists an index } h \in\{1, \ldots, i-1\} \text { with } \gamma_{h}=j \\ =\bar{\theta}_{j} & \text { otherwise. }\end{cases}$

Since $v^{i}=v^{1}+\sum_{j=1}^{i-1} q\left(\gamma_{j}\right) / d_{m}$ it follows that

$$
v^{i}=v(\bar{\rho})+\sum_{j=1}^{n+1} \theta_{j}^{i} q(j) / d_{m} \quad i=1, \ldots, n+1 .
$$

By (8.4.1) and (8.4.2) we have that $\theta_{j}^{i} \geq 0$ for all $i, j$. Let $H\left(v^{i}\right)$ be the set of indices $\left\{j \mid \Theta_{j}^{i}=0\right\}, i=1, \ldots, n+1$. By definition, $\left|H\left(v^{1}\right)\right| \geq 1,\left|H\left(v^{n+1}\right)\right| \leq 1$ and $\left|H\left(v^{i}\right)\right|-\left|H\left(v^{i+1}\right)\right|$ is equal to zero or one. So there exists at least one index $\mathrm{h}$ such that

$$
\left|\mathrm{H}\left(\mathrm{v}^{\mathrm{h}}\right)\right|=1 \text {. }
$$


Let $s$ be the index such that $\{s\}=H\left(v^{h}\right)$ for some $h$ with $\left|H\left(v^{h}\right)\right|=1$. Since $H\left(v^{i+1}\right) \subseteq H\left(v^{i}\right), s$ is unique. Let $i_{0}$ be the index such that $\gamma_{i_{0}}=s$. Define

$$
\begin{aligned}
& \bar{T}=I_{n+1} \backslash\left\{\gamma_{i_{0}}\right\} \\
& \bar{\pi}(\bar{T})=\left\{\gamma_{i_{0}+1}, \ldots, \gamma_{n+1}, \gamma_{1}, \ldots, \gamma_{i_{0}-1}\right\} \\
& \bar{R}_{j}=\Theta_{j}^{i_{0}+1}-\min _{i \in I} \Theta_{i}^{i_{0}+1}{ }_{i} \quad j=1, \ldots, n+1 .
\end{aligned}
$$

It can easily be seen that $\bar{R}_{j}=0$ for $j=\gamma_{i_{0}}$, i.e. $\bar{R}_{j}=0$ for $j \notin \bar{T}$. Moreover $\bar{R}_{j} \geq 0, j \in \bar{T}$. Define $y^{1}$ to be $v^{j 0^{1}}$. Then ${ }^{i_{0}} \tau\left(v^{1}, \gamma\left(I_{n+1}\right)\right)$ can be written as $\bar{\sigma}\left(y^{1}, \bar{\pi}(\bar{T})\right)$. Since

$$
y^{1}=v^{i} 0^{+1}=v(\bar{\rho})+\sum_{j=1}^{n+1} \bar{R}_{j} q(j) / d_{m}
$$

it follows that $\bar{\sigma}$ is in $A^{\bar{\rho}}(\bar{T})$. So, finally we have that $\tau$ becomes $\bar{\rho}\left(\bar{z}^{-1}, \bar{\beta}\left(I_{n+1}\right)\right), T$ becomes $\bar{T}, \pi(T)$ becomes $\bar{\pi}(\bar{T})$ and $R$ becomes $\bar{R}_{\text {, }}$ i.e. the only vertex $y^{1}$ of $\sigma$ on level $d_{m+1}$ is replaced by the vertex $\bar{z}^{j_{0}}$ of $\bar{\rho}$ on level $d_{m-1}$, where $j_{0}$ is the index such that $\bar{\beta}_{j_{0}}=s$. The zero-dimensional simplex $\sigma$ on level $d_{m+1}$ becomes the $n$-dimensional simplex $\bar{\sigma}$ on level $d_{m}$.

\subsection{THE APPLICATION ON $\mathrm{R}^{\mathrm{n}}$}

Let $d_{0}, d_{1}, \ldots$ be a sequence of increasing positive numbers such that $d_{i+1}=k_{i} d_{i}, i=0,1, \ldots$ with $k_{i}$ an arbitrary integer larger than 1 . For some $n \times n$-triangulation matrix $A, R^{n} \times\left\{d_{m}\right\}$ is triangulated by the AK triangulation with grid size $d_{m}^{-1}$. On each level we choose $\mathrm{z}=\underline{0}$ to be a grid point. Doing so, the grid points of the triangulation on level $\mathrm{d}_{\mathrm{m}}$ are also a grid point on level $d_{i}, i>m$. With $a(n+1)=-\sum_{j=1}^{n} a(j)$ we obtain again that each $n$-simplex $\sigma$ has $n+1$ representations $\sigma\left(y^{1}, \pi\left(I_{n+1}\right)\right)$. As in section 2 each simplex is given in the s-representation. Therefore we have to define the function $s(w)$. So, let $w$ be a grid point on level $d_{m}$. Then we define $\alpha(w)$ by

$$
\alpha(w)=d_{m} A^{-1} w
$$

and $s(w)$ by $s(w)=1+\left(\sum_{h=1}^{n} \alpha_{h}(w)\right) \bmod (n+1)$. 
Now we triangulate $R^{n} \times\left[d_{m}, d_{m+1}\right]$ for some $m \geq 0$. Combining these triangulations for all palirs $\left[d_{i}, d_{i+1}\right], i=0,1, \ldots$ we obtain the triangulation of $R^{n} \times\left[d_{0}, \infty\right)$. The triangulation of $R^{n} \times\left[d_{m}, d_{m+1}\right]$ is done in the same way as described in section 2 for $s^{n} \times\left[d_{m} d_{m+1}\right]$. So, let $\sigma_{m}\left(u^{1}, \beta\left(I_{n+1}\right)\right)$ be the starting simplex on level $d_{m}$, and let

$$
v\left(\sigma_{m}\right)=\sum_{i=1}^{n+1} k_{m}^{-1} \lambda_{i}^{m} u^{i}
$$

be its centrepoint for arbitrarily chosen $\lambda_{i^{\prime}}^{m} i \in I_{n+1^{\prime}}$ such that $\sum_{i=1}^{n+1} \lambda_{i}^{m}=k_{m}$. To triangulate $\tau\left(\mathrm{y}^{1}, \pi\left(\mathrm{I}_{\mathrm{n}+1}\right)\right) \times\left[\mathrm{d}_{\mathrm{m}}, \mathrm{d}_{\mathrm{m}+1}\right]$, with $\tau$ given in the s-representation we define again for any proper subset $T$ of $I_{n+1}$, the regions $\AA^{\tau}(T)$ as in section 2 , and connect all grid points $x$ on level $d_{m+1}$ in region $\AA^{\tau}(T)$ with the vertices $y^{i}$ of $\tau$ on level $d_{m}$ with $i \notin T$. Combining the triangulations $\tau \times\left[d_{m}, d_{m+1}\right]$ over all simplices $\tau$, we get a consistent triangulation of $R^{n} \times\left[d_{m}, d_{m+1}\right]$.

We now discuss the use of this triangulation of $R^{n} \times\left[d_{0}, \infty\right)$ in a fixed point algorithm. Assume we want to compute a fixed point of a mapping $\phi$. Let $f^{i}$ be a piecewise linear approximation to $\phi$ with respect to the triangulation of $R^{n}$ on level $d_{i}$. Then a point $x$ of $R^{n} \times\left\{d_{i}\right\}$ receives the vector label as defined in definition 4.8.1 with $b=e$ and $f=f^{i}, i=0,1, \ldots$. Assume that Merrill's condition is satisfied and that $R^{n} \times\left\{d_{i}\right\}$ is triangulated by $A(\alpha) K_{\delta_{i}}$ with $\delta_{i}=d_{i}^{-1}, i=0,1, \ldots$. Let $\sigma^{0}$ be a completely labelled simplex on level $d_{0}$. Then, starting with the simplex having $\sigma^{0}$ as a facet, a sequence of simplices of the triangulation of $R^{n} \times\left[d_{0}, \infty\right)$ is generated, such that the common facets are completely labelled. By Merrill's condition this sequence cannot diverge if $\delta_{0}$ is small enough. If, for some m large enough, a completely labelled simplex of $R^{n} \times\left\{d_{m}\right\}$ is generated, a good approximation of a fixed point is found and the algorithm terminates. It remains to consider the following questions:

$1^{e}$. How to find a completely labelled simplex $\sigma^{0}$ in $R^{n} \times\left\{d_{0}\right\}$ ?

$2^{e}$. How to continue the algorithm if, starting from the simplex having $\sigma^{0}$ as a facet, a simplex of $R^{n} \times\left[d_{0}, a_{1}\right]$ is generated having a completely labelled facet $\sigma\left(y^{1}, \pi\left(I_{n+1}\right)\right)$ in $R^{n} \times\left\{d_{0}\right\}$ ?

Both problems can be solved by applying the variable dimension restart algorithm. Starting in an arbitrarily chosen grid point $\left(v^{\top}, d_{0}\right)^{\top}$ in $R^{n} \times\left\{d_{0}\right\}$, this algorithm generates a sequence of simplices of variable 
dimension on level $d_{0}$. By theorem 6.3.4 this sequence terminates with a completely labelled simplex $\sigma^{0}$ in $R^{n} \times\left\{d_{0}\right\}$. To solve the second question, observe that, for some $i \in I_{n+1}, \sigma\left(y^{1}, \pi\left(I_{n+1}\right)\right)$ is in the region

$$
A(T)=\left\{x \in R^{n} \mid x=v+\sum \lambda_{j} a(j) \text { for nonnegative numbers } \lambda_{j^{\prime}} j \in T\right\}
$$

with $T=I_{n+1} /\{i\}$. Then the variable dimension restart algorithm can again be applied. Starting with $\sigma$ and reintroducing the $i$-th unit column, a sequence of simplices of variable dimension in level $d_{0}$ is generated until again a completely labelled simplex is found. Then the algorithm continues again with simplices of the triangulation of $R^{n} \times\left[d_{0}, \infty\right)$. In fact we can use the interpretation of the variable dimension restart algorithm with $n+1$ points on an extra level, as discussed in section 6.4, to triangulate for some $\sigma$ $(\sigma \times\{0\}) \cup\left(R^{n} \times\left(0, d_{0}\right]\right)$. Then, starting with the simplex being the convex hull of the vertices of $\sigma$ on level 0 and $\left(v^{\top}, d_{0}\right)^{\top}$, a sequence of $n+1$-simplices having completely labelled common facets is generated.

\subsection{SOME REMARKS AND NUMERICAL RESULTS.}

In the algorithm of Eaves [1972] and Eaves and Saigal [1972] the factor of grid refinement is (at most) equal to two. So, their algorithm cannot be used to obtain quadratic convergence. Using a restart method in a continuous deformation algorithm, Saigal [1977c] and saigal and Todd [1978] developed an acceleration technique to achieve quadratic convergence when the underlying function is continuously differentiable and the derivative is Lipschitz continuous. Using the triangulation presented in this chapter, quadratic convergence can be obtained without the necessity of making restarts. Saigal and Todd also proved that, using the information obtained from the last found approximation, a completely labelled simplex on the new level can be generated in $n+1$ pivots. It is worthwile to investigate whether the same result can be proved for the continuous deformation algorithm with an arbitrary factor of grid refinement.

In the triangulations of $S^{n} \times[1, \infty)$ and $R^{n} \times\left[d_{0}, \infty\right)$, described in section 2 respectively 5 , the starting point on a new level can be chosen on basis of information obtained earlier. So, for vector labelling one could choose the grid point nearest to the approximation obtained from the last found completely labelled simplex. Therefore, even when 
the factor of incrementation is two, the triangulation is more general than that of Eaves and Saigal. This is illustrated in the figures 8.6.1.a and 8.6.1.b for $\mathrm{n}=1$. In 8.6.1.a, $\tau\left(\mathrm{y}^{1}, \mathrm{y}^{2}\right) \times\left[\mathrm{d}_{\mathrm{m}^{\prime}}, \mathrm{d}_{\mathrm{m}}\right]$ is triangulated according to the triangulation of Eaves and Saigal. Observe that $y^{i} \times\left\{d_{m}\right\}, i=1,2$, must be connected with $\mathrm{v} \times\left\{2 \mathrm{~d}_{\mathrm{m}}\right\}$. In $8.6 .1 . \mathrm{b}, \tau\left(\mathrm{y}^{1}, \mathrm{y}^{2}\right) \times\left[\mathrm{d}_{\mathrm{m}}, 2 \mathrm{~d}_{\mathrm{m}}\right]$ is triangulated by connecting $y^{i} \times\left\{d_{m}\right\}, i=1,2$ with $y^{1} \times\left\{2 d_{m}\right\}$, and $y^{2} \times\left\{d_{m}\right\}$ with the other two grid points of $\tau \ddot{i}\left\{2 d_{m}\right\}$. Observe however that the vertices $y^{i} \times\left\{d_{m}\right\}, i=1,2$, can be connected with any grid point of $\tau \times\left\{2 d_{m}\right\}$.

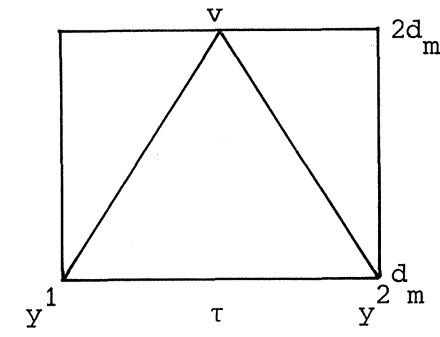

Figure 8.6.1.a Eaves-Saigal's triangulation of $\tau \times\left[\mathrm{d}_{\mathrm{m}}, 2 \mathrm{~d}_{\mathrm{m}}\right]$

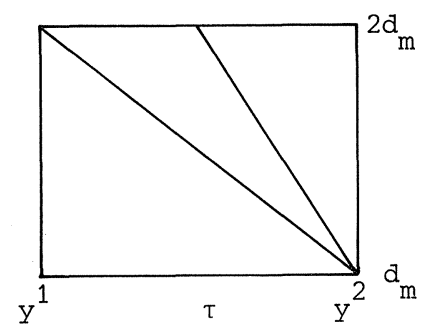

Figure 8.6.1.b. Example of a triangulation of $\tau \times\left[\mathrm{d}_{\mathrm{m}}, 2 \mathrm{~d}_{\mathrm{m}}\right]$ as given in section 2 .

Tuy [1979] presented a restart method to approximate a fixed point of a function, using primitive sets (see also Todd [1978b]). Although his approach is different, Van der Heyden [1979b] presented a similar method. He introduced also a geometric interpretation of the algorithm. Using this interpretation he constructed a triangulation of $S^{n} \times[1, \infty)$ permitting an arbitrary factor of grid refinement between two successive levels. However, this is achieved at the expense of an asymmetry in the roles played by the different coordinates. More precisely, $\tau\left(y^{1}, \ldots, y^{n+1}\right) \times\left[d_{m}, d_{m+1}\right]$ is triangulated as in section 2, but with the restriction that, for some $i, v=y^{i}$. Although a partial remedy is given, this feature makes the triangulation less attractive. The difference between Van der Heyden's triangulation and the triangulation introduced in section 2 is illustrated in the figures 8.6.2.a,b. Finally we mention a paper of Shamir [1979]. Independently of Van der Laan and Talman [1980b] he constructed the same triangulation of $S^{n} \times[1, \infty)$ using also the interpretation of the variable dimension restart algorithm with an extra level. 


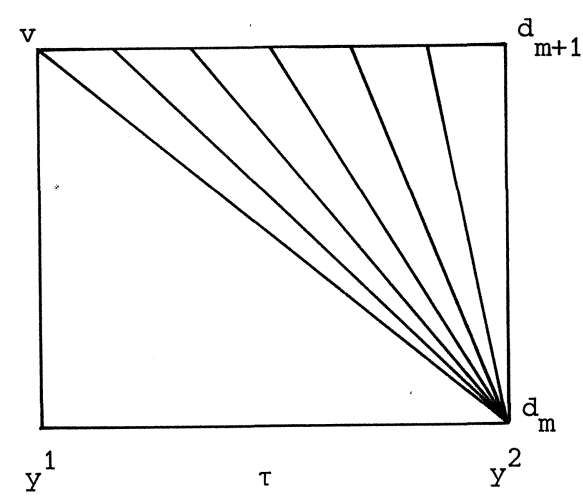

Figure 8.6.2.a. Van der Heyden's

triangulation of $\tau \times\left[d_{m}, d_{m+1}\right], n=1$, $\mathrm{k}_{\mathrm{m}}=6, \mathrm{v}=\mathrm{y}^{1}$ or $\mathrm{y}^{2}$.

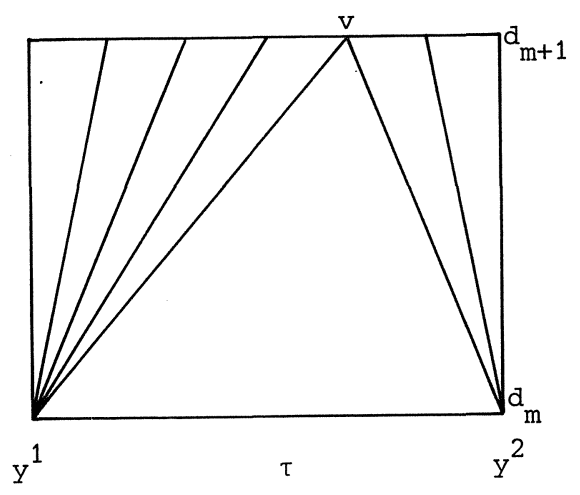

Figure 8.6.2.b. Example of a triangulation of $\tau \times\left[d_{m}, d_{m+1}\right]$ as given in section $2, n=1, k_{m}=6, v$ is an arbitrary grid point on level $d_{m+1}$.

We conclude this section with some computational results. We applied the algorithm for vector labelling to the three examples of section 5.8. In all runs the $T$ triangulation is used with again $-T$ in stead of $T$. The labelling rule is the same as in section 5.8. The starting point on level $\mathrm{d}_{\mathrm{m}+1}$ is chosen as the grid point nearest to $\mathrm{w}^{*}(\mathrm{~m})$ with $\mathrm{w}^{*}(\mathrm{~m})$ the approximate fixed point on level $d_{m}$. The algorithm is applied with $d_{i+1} / d_{i}=2$ for all $i$, and with a variable factor of grid refinement. In the latter case $\delta_{m+1}=d_{m+1}^{-1}$ is chosen analogously as in section 5.8 . However to guarantee that $d_{m+1}$ is a multiple of $d_{m^{\prime}} \delta_{m+1}$ is defined by $\delta_{m+1}=\delta_{m} / k_{m}$ with

$$
\begin{array}{ll}
k_{m}=2, & \text { if } h_{m} \geq\left(\operatorname{mesh} G_{m}\right) / 2 \sqrt{n+1} \\
k_{m}=\text { Entier }\left(h_{m} \sqrt{n+1} / \text { mesh } G_{m}\right)^{-1} \text { if } h_{m}<\left(\operatorname{mesh} G_{m}\right) / 2 \sqrt{n+1}
\end{array}
$$

The results are given in the tables 8.6.1-8.6.3. Comparing the columns 3 and 7 it turns out that the algorithm is considerably improved by using a variable factor of grid refinements. Moreover a comparison with the tables 5.8.1-5.8.3 learns that for $d_{i+1} / d_{i}=k_{i}$ the number of iterations of the continuous deformation algorithm is of the same order as the number of iterations of the variable dimension restart algorithm. More 
computational experience is needed to decide which algorithm is superior. We remark the following:

1. The variable dimension restart algorithm is easier to implement on the computer.

2. In each stage the restart algorithm is initiated with $I \mu=e$. This is a drawback, since the deformation algorithm continues with the current system of linear equations. However in the acceleration technique of Todd [1978b] the system of linear equations corresponding to the completely labelled simplex is used to initiate the system in the next stage. This technique should be used when the restart algorithm is compared with the deformation algorithm.

3. The examples are very nice. In particular, the deformation algorithm never returns to a previous level. Both algorithms should be tested on more complicated problems.

Table 8.6.1. Pure exchange economy, $n=4, M=\left(\operatorname{mesh} G_{m}\right) / 2 \sqrt{n+1}$, $N$ is the cumulative number of iterations, $E=\max \left|\left(g_{i}-a_{i}\right) / a_{i}\right|$.

$$
i \in I_{n+1}
$$

\begin{tabular}{|c|c|c|c|c|c|c|c|}
\hline m & M & $\mathrm{N}$ & $E$ & $\mathrm{~m}$ & M & $\mathrm{N}$ & $\mathrm{E}$ \\
\hline 1 & .051 & 7 & 1.02 & 1 & .051 & 7 & 1.02 \\
\hline 3 & .013 & 20 & .05 & 2 & .026 & 13 & .29 \\
\hline 5 & .003 & 33 & $810^{-3}$ & 3 & .013 & 20 & .05 \\
\hline 7 & $810^{-4}$ & 43 & $210^{-4}$ & 4 & .006 & 26 & .03 \\
\hline 9 & $210^{-4}$ & 55 & $2.510^{-5}$ & 5 & $2^{-} 10^{-3}$ & 31 & $310^{-3}$ \\
\hline 11 & $510^{-5}$ & 69 & $210^{-6}$ & 6 & $10^{-3}$ & 38 & $10^{-3}$ \\
\hline 13 & $1.210^{-5}$ & 81 & $310^{-8}$ & 7 & $810^{-5}$ & 45 & $410^{-6}$ \\
\hline 15 & $310^{-6}$ & 91 & $510^{-9}$ & 8 & $410^{-5}$ & 52 & $210^{-6}$ \\
\hline 17 & $810^{-7}$ & 102 & $410^{-10}$ & 9 & $610^{-8}$ & 59 & $<10^{-10}$ \\
\hline
\end{tabular}


Table 8.6.2. Pure exchange economy, $n=7, M=\left(\right.$ mesh $\left.G_{m}\right) / 2 \sqrt{n+1}, N$ is the cumulative number of iterations, $E=\max \left|\left(g_{i}-a_{i}\right) / a_{i}\right| \cdot$ $i \in I_{n+1}$

\begin{tabular}{|c|c|c|c|c|c|c|c|}
\hline \multicolumn{4}{|c|}{$d_{i+1} / d_{i}=2$} & \multicolumn{4}{|c|}{$d_{i+1} / d_{i}=k_{i}$} \\
\hline $\mathrm{m}$ & $M$ & $\mathrm{~N}$ & $E$ & $\mathrm{~m}$ & $M$ & $\mathrm{~N}$ & $E$ \\
\hline 1 & .032 & 11 & 1.32 & 1 & .032 & 11 & 1.32 \\
\hline 3 & .008 & 36 & .05 & 2 & .016 & 27 & .23 \\
\hline 5 & .002 & 59 & $510^{-3}$ & 3 & .008 & 36 & .05 \\
\hline 7 & $510^{-4}$ & 83 & $210^{-4}$ & 4 & $3.810^{-3}$ & 46 & .02 \\
\hline 9 & $1.210^{-4}$ & 102 & $10^{-5}$ & 5 & $5.610^{-4}$ & 62 & $210^{-4}$ \\
\hline 11 & $310^{-5}$ & 118 & $10^{-6}$ & 6 & $2.810^{-4}$ & 70 & $510^{-5}$ \\
\hline 13 & $810^{-6}$ & 142 & $810^{-8}$ & 7 & $3.210^{-6}$ & 80 & $210^{-8}$ \\
\hline 15 & $210^{-6}$ & 166 & $410^{-9}$ & 8 & $1.610^{-6}$ & 93 & $410^{-9}$ \\
\hline
\end{tabular}

Table 8.6.3. Pure exchange economy, $n=9, M=\left(\right.$ mesh $\left.G_{m}\right) / 2 \sqrt{n+1}, N$ is the cumulative number of iterations, $E=\max _{i \in I}\left|\left(g_{i}-a_{i}\right) / a_{i}\right|$.

\begin{tabular}{|c|l|l|l|l|l|l|l|}
\hline $\mathrm{m}$ & $\mathrm{M}$ & $\mathrm{N}$ & $\mathrm{E}$ & \multicolumn{4}{|c|}{$\mathrm{d}_{i+1} / \mathrm{d}_{i}=\mathrm{k}_{i}$} \\
\hline 1 & .025 & 12 & .30 & 1 & .025 & 12 & .30 \\
3 & .006 & 43 & .03 & 2 & .013 & 32 & .08 \\
5 & $1.510^{-3}$ & 83 & $210^{-3}$ & 3 & .006 & 43 & .03 \\
7 & $410^{-4}$ & 112 & $10^{-4}$ & 4 & .002 & 67 & $310^{-3}$ \\
9 & $10^{-4}$ & 142 & $610^{-6}$ & 5 & $710^{-4}$ & 78 & $310^{-4}$ \\
11 & $2.510^{-5}$ & 166 & $410^{-7}$ & 6 & $510^{-5}$ & 102 & $10^{-6}$ \\
13 & $610^{-6}$ & 193 & $310^{-8}$ & 7 & $610^{-6}$ & 120 & $310^{-8}$ \\
\hline
\end{tabular}


REFERENCES

[1] Allgower, E.L. and Georg, K. (1979), Generation of triangulations by reflections,

Utilitas Mathematica 16, pp. 123-129.

[2] Allgower, E.L. and Georg, K. (1980), Simplicial and continuation methods for approximating fixed points and solutions to systems of equations, SIAM Rev. 22, pp. 28-75.

[3] Arrow, K.J. and Hahn, F.H. (1971), General competitive analysis, Holden-Day, San Francisco.

[4] Bárány, I. (1979a), Subdivisions and triangulations in fixed point algorithms, International Research Institute for Management Science, ul. Ryleeva, Preprint, Moscow.

[5] Bárány, I. (1979b), private communication.

[6] Berge, C. (1966), Espaces topologiques, Dunod, Paris.

[7] Brouwer, L.E.J. (1912), Tber Abbildung von Mannigfaltigkeiten, Math. Ann. 71, pp. 97-115.

[8] Charnes, A., Garcia, C.B. and Lemke, C.E. (1977), Constructive proofs of theorems relating to $f(x)=y$, with applications, Math. Programming 12, pp. 328-343.

[9] Colville, A.R. (1968), A comparative study on nonlinear programming codes, IBM New York Scientific Center Report No. 320-2949.

[10] Dantzig, G.B. (1951), Maximization of a linear function of variables subject to linear inequalities, Activity analysis of production and allocation, T.C. Koopmans, ed., John Wiley, New York, pp. 339-347.

[11] Dantzig, G.B. (1963), Linear programming and extensions, Princeton University Press, Princeton.

[12] Debreu, G. (1959), Theory of value, John Wiley, New York.

[13] Drèze, J. (1975), Existence of an exchange equilibrium under price rigidities, Internat. Econom. Rev. 16, pp. 301-320.

[14] Eaves, B.C. (1971), Computing Kakutani fixed points, SIAM J. Appl. Math. 21, pp. 236-244.

[15] Eaves, B.C. (1972), Homotopies for computation of fixed points, Math. Programming 3 , pp. 1-22.

[16] Eaves, B.C. (1976), A short course in solving equations with $P L$ homotopies, SIAM-AMS Proceedings 9, pp. 73-143. 
[17] Eaves, B.C. and Saigal, R. (1972), Homotopies for computation of fixed points on unbounded regions, Math. Programming 3 , pp. 225-237.

[18] Eaves, B.C. and Scarf, H.E. (1976), The solution of systems of piecewise linear equations, Math. Oper. Res. 1, pp. 1-27.

[19] Fisher, M.L., Gould, F.J. and Tolle, J.W. (1977), A new simplicial approximation algorithm with restarts: relations between convergence and labellings, Fixed points: algorithms and applications, S. Karamardian, ed., Academic Press, New York, pp. 41-58.

[20] Freudenthal, H. (1942), Simplizialzerlegungen von beschränkter Flachheit, Ann. of Math. 43, pp. 580-582.

[21] Garcia, C.B. (1975), A global existence theorem for the equation $F \mathrm{x}=y$, Center for Mathematical Studies in Business and Economics Report 7527, University of Chicago, Chicago.

[22] Garcia, C.B. and Gould, F.J. (1976), An algorithm based on the equivalence of vector and scalar labels in simplicial approximation, Center for Mathematical Studies in Business and Economics Report 7626, University of Chicago, Chicago.

[23] Garcia, C.B. and Gould, F.J. (1978), A theorem on homotopy paths, Math. Oper. Res. 3, pp. 282-289.

[24] Garcia, C.B. and Gould, F.J. (1979), Scalar labellings for homotopy paths, Math. Programming 17, pp. 184-197.

[25] Garcia, C.B. and Zangwill, W.I. (1978), on a new approach to homotopy and degree theory, Center for Mathematical studies in Business and Economics Report 7813, University of Chicago, Chicago.

[26] Gould, F.J. and Tolle, J.W. (1974), A unified approach to complementarity in optimization, Discrete Math. $\underline{7}, \mathrm{pp}$. 225-271.

[27] Gould, F.J. and Tolle, J.W. (1975), Finite and constructive conditions for a solution to $f(x)=0$, Center for Mathematical Studies in Business and Economics Report 7515, University of Chicago, Chicago.

[28] Greenberg, J. (1977), Quasi-equilibrium in abstract economies without ordered preferences, J. Math. Econom. 4, pp. 163-166.

[29] Hansen, T. (1968), On the approximation of a competitive equilibrium, Ph.D. Dissertation, Yale University, New Haven. 
[30] Hansen, T. and Scarf, H. (1969), On the applications of a recent combinatorial algorithm, Cowles Foundation Discussion Paper 272, Yale University, New Haven.

[31] Heyden, L. van der (1979a), Refinement methods for computing fixed points using primitive sets, Ph.D. Dissertation, Yale University, New Haven.

[32] Heyden, L. van der (1979b), Restricted primitive sets in a regularly distributed list of vectors and simplicial subdivisions with arbitrary refinement factors, John F. Kennedy School of Government,'Preprint, Harvard University, Cambridge (Mass.).

[33] Jeppson, M.M. (1972), A search for the fixed points of a continuous mapping, Mathematical topics in economics theory and computation, R.H. Day and S.M. Robinson, eds., SIAM, Philadelphia, pp. 122-129.

[34] Kakutani, S. (1941), A generalization of Brouwer's fixed point theorem, Duke Math. J. 8 , pp. 457-459.

[35] Karamardian, S. (1977), ed., Fixed points: algorithms and applications, Acadenic Press, New York.

[36] Kellogg, R.B., Li, T.Y. and Yorke, J. (1976), A constructive proof of the Brouwer fixed point theorem and computational results, SIAM J. Numer. Anal. 4 , pp. 473-483.

[37] Knaster, B., Kuratowski, C. and Mazurkiewicz, S. (1929), Ein Beweis des Fixpunktsatzes für n-dimensionale Simplexe, Fund. Math. 14 , pp. 132-137.

[38] Kojima, M. (1978a), on the homotopic approach to systems of equations with separable mappings, Math. Programming stud. , pp. 170-184.

[39] Kojima, M. (1978b), A modification of Todd's triangulation $J_{3}$, Math. Programming 15 , pp. 223-227.

[40] Kojima, M. (1980), A note on "A new algorithm for computing fixed points" by Van der Laan and Talman. Numerical solution of highly nonlinear problems, w. Foster, ed., North-Holland, Amsterdam, pp. 37-42.

[41] Kuhn, H.W. (1960), Some combinatorial lemmas in topology, I.B.M. J. Res. Develop. 4, pp. 518-524.

[42] Kuhn, H.W. (1968), Simplicial approximation of fixed points. Proc. Nat. Acad. Sci. U.S.A. 61, pp. 1238-1242.

[43] Kuhn, H.W. (1969), Approximate search for fixed points, Computing methods in optimization problems 2, Academic Press, New York. 
[44] Kuhn, H.W. and Mackinnon, J.G. (1975), Sandwich method for finding fixed points, J. Optimization Theory Appl. 17, pp. 189-204.

[45] Laan, G. van der (1980), Equilibrium under rigid prices with compensation for the consumers, Internat.Econom. Rev. 21, pp. 63-73.

[46] Laan, G. van der, and Talman, A.J.J. (1978.a), On the computation of fixed points in the product space of unit simplices and an application to non cooperative $N$ person games, Interfaculteit der Actuariële Wetenschappen en Econometrie, Onderzoekverslag 35, Vrije Universiteit, Amsterdam.

[47] Laan, G. van der, and Talman, A.J.J. (1978b), A class of simplicial subdivisions for restart fixed point algorithms without an extra dimension, Interfaculteit der Actuariële Wetenschappen en Econometrie, Onderzoekverslag 36, Vrije Universiteit, Amsterdam, to appear in Math. Programming.

[48] Laan, G. van der, and Talman, A.J.J. (1979a), A restart algorithm for computing fixed points without an extra dimension, Math. Programming 17, pp. 74-84.

[49] Laan, G. van der, and Talman, A.J.J. (1979b), A restart algorithm without an artificial level for computing fixed points on unbounded regions, Functional differential equations and approximation of fixed points, H.-O. Peitgen and H.-O. Walther, eds., Lecture Notes in Mathematics 730, Springer-Verlag, Berlin, pp. 247-256.

[50] Laan, G. van der, and Talman, A.J.J. (1979C), Interpretation of the variable dimension fixed point algorithm with an artificial level, Interfaculteit der Actuariële Wetenschappen en Econometrie, Onderzoekverslag 47, Vrije Universiteit, Amsterdam.

[51] Laan, G. van der, and Talman, A.J.J.. (1980a), An improvement of fixed point algorithms by using a good triangulation, Math. Programming 18, pp. 274-285.

[52] Laan, G. van der, and Talman, A.J.J. (1980b), A new subdivision for computing fixed points with a homotopy algorithm, Math. Programming 19, pp. 78-91. 
[53] Laan, G. van der, and Talman, A.J.J. (1980c), Convergence and properties of recent variable dimension algorithms, Numerical solution of highly nonlinear problems, w.Foster, ed., North-Holland, Amsterdam, pp. 3-36.

[54] Lefschetz, S. (1949), Introduction to topology, Princeton University Press, Princeton.

[55] Lemke, C.E. (1965), Bimatrix equilibrium points and mathematical programming, Management Sci. 11, pp. 681-689.

[56] Lemke, C.E. and Grotzinger, S.J. (1976), on generalizing Shapley's index theory to labelled pseudo manifolds, Math. Programming 10, pp. 245-262.

[57] Lemke, C.E. and Howson jr., J.T. (1964), Equilibrium points of bimatrix games, SIAM J. Appl. Math. 12, pp. 413-423.

[58] Lüthi, H.J. (1975), A simplicial approximation of a solution for the nonlinear complementarity problem, Math. Programming $\underline{9}$, pp. 278-293.

[59] Lüthi, H.J. (1976), Komplementaritats- und Fixpunktalgorithmen in der mathematischen Programmierung, spieltheorie und Okonomie, Lecture Notes in Economics and Mathematical Systems 129, Springer-Verlag, Berlin.

[60] Mackinnon, J.G. (1976), The existence and computation of equilibria with increasing returns and externalities, Department of Economics Preprint 217, Queen's University, Kingston (Can.).

[61] Mackinnon, J.G. (1977), Solving economic general equilibrium models by the Sandwich method, Fixed points: algorithms and applications, S. Karamardian, ed., Academic Press, New York, pp. 367-402.

[62] Mara, P.S. (1972), Triangulations of a cube, M.S. Thesis, Colorado State University, Fort Collins. 
[63] Merrill, O.H. (1971), Applications and extensions of an algorithm that computes fixed points of certain non-empty, convex, upper semi-continuous point to set mappings, Department of Industrial Engineering, Technical Report 71-7, University of Michigan, Ann Arbor.

[64] Merrill, O.H. (1972), Applications and extensions of an algorithm that computes fixed points of certain upper semi-continuous point to set mappings, Ph. D. Dissertation, University of Michigan, Ann Arbor.

[65] Peitgen, H.-O. and Prüfer, M. (1979), The Leray-Schauder continuation method is a constructive element in the numerical study of nonlinear eigen value and bifurcation problems, Functional differential equations and approximation of fixed points, H.-O. Peitgen and H.-O. Walther, eds., Lecture Notes in Mathematics 730, Springer-Verlag, Berlin, pp. 326-409.

[66] Peitgen, H.-O. and Walther, H.-O. (1979), eds., Functional differential equations and approximation of fixed points, Lecture Notes in Mathematics 730, Springer-Verlag, Berlin.

[67] Prüfer, M. (1977), Sperner simplices and the topological fixed point index, Institut für Angewandte Mathematik, Preprint 134, Universität Bonn, Bonn.

[68] Prüfer, M. and Siegberg, H.W. (1979), On computational aspects of topological degree in $R^{n}$, Functional differential equations and approximation of fixed points, H.-O. Peitgen and H.-O. Walther, eds., Lecture Notes in Mathematics 730, SpringerVerlag, Berlin, pp. 410-433.

[69] Reif, N. (1978), The computation of Drèze equilibria, Department of Economics, Preprint, Universität Hamburg, Hamburg.

[70] Reiser, P.M. (1978a), Ein hybrides Verfahren zur Lb̈sung von nichtlinearen Komplementaritytsproblemen und seine Konvergenzeigenschaften, Dissertation, Eidgenössischen Technischen Hochschule Zürich, Zürich.

[71] Reiser, P.M. (1978b), Convergence properties of complementarity algorithms with integer labelling, Institut für operations Research, Manuskripte, Universität zürich, zürich.

[72] Richter, D.K. (1978), The computation of a general equilibrium in a public good economy, Math. Programming 14, pp. 186-207. 
[73] Rockafellar, R.T. (1970), Convex analysis, Princeton University Press, Princeton.

[74] Saari, D.G. and Saigal R. (1979), Some generic properties of paths generated by fixed point algorithms, Department of Industrial Engineering and Management Science, Preprint, Northwestern University, Evanston, to appear in the proceedings of the Symposium on Analysis and Computation of Fixed Points, Madison.

[75] Saigal, R. (1976), On paths generated by fixed point algorithms, Math. Oper. Res. 1, pp. 359-380.

[76] Saigal, R. (1977a), Investigations into the efficiency of fixed point algorithms, Fixed points: algorithms and applications, S. Karamardian, ed., Academic Press, New York, pp. 203-223.

[77] Saigal, R. (1977b), Fixed point computing methods, Encyclopedia of computer science and technology 8 , pp. 213-235.

[78] Saigal, R. (1977c), on the convergence rate of algorithms for solving equations that are based on methods of complementary pivoting, Math. Oper. Res. 2 , pp. 108-124.

[79] Saigal, R. (1979a), The fixed point approach to nonlinear programming, Math. Programming Stud. 10, pp. 142-157.

[80] Saigal, R. (1979b), on piecewise linear approximations to smooth mappings, Math. Oper. Res. 4, pp. 153-161.

[81] Saigal, R., Solow, D. and Wolsey, L.A. (1975), A comparative study of two algorithms to compute fixed points over unbounded regions, presented at the 8 th Math. Programming Symposium, Stanford.

[82] Saigal, R. and Todd, M.J. (1978), Efficient acceleration techniques for fixed point algorithms, SIAM J. Numer. Anal. 15, pp. 997-1007.

[83] Scarf, H. (1967a), The core of an $N$-person game, Econometrica 37, pp. 50-69.

[84] Scarf, H. (1967b), The approximation of fixed points of a continuous mapping, SIAM J. Appl. Math. 15, pp. 1328-1343.

[85] Scarf, H.E. (1973), with the collaboration of T. Hansen, Computation of economic equilibria, Yale University Press, New Haven. 
[86] Shamir, S. (1979), A homotopy fixed point algorithm with an arbitrary integer refinement factor, Department of Engineering-Economic Systems, Report SE-79R-2, Stanford University, Stanford.

[87] Shapley, L.S. (1974), A note on the Lemke-Howson algorithm, Math. Programming Stud. 1 , pp. 175-189.

[88] Shoven, J.B. and Whalley, J. (1973), General equilibrium with taxes: a computational procedure and an existence proof, Rev. Econ. Studies 40, pp. 475-489.

[89] Siegberg, H.W. (1980), Brouwer degree:history and numerical computation, Numerical solution of highly nonlinear problems, W. Foster, ed., North Holland, pp. 389-407.

[90] Spanier, E.H. (1966), Algebraic topology, MacGraw-Hill, New York.

[91] Sperner, E. (1928), Neuer Beweis fur die Invarianz der Dimensionszahl und des Gebietes, Abh. Math. Sem. Univ. Hamburg 6, pp. 265-272.

[92] Talman, A.J.J. (1980), Variable dimension fixed point algorithms and triangulations, Dissertation, Vrije Universiteit, Amsterdam.

[93] Todd, M.J. (1976a), The computation of fixed points and applications, Lecture Notes in Economics and Mathematical Systems 124, Springer-Verlag, Berlin.

[94] Todd, M.J. (1976b), on triangulations for computing fixed points, Math. Programming 10, pp. 322-346.

[95] Todd, M.J. (1976c), Orientation in complementary pivot algorithms, Math. Oper. Res.1, pp. 54-66.

[96] Todd, M.J. (1977a), Union Jack triangulations, Fixed points: algorithms and applications, s. Karamardian, ed., Academic Press, New York, pp. 315-336.

[97] Todd, M.J. (1977b), New fixed point algorithms for economic equilibria and constrained optimization, School of Operations Research and Industrial Engineering, Technical Report 362, Cornell University, Ithaca.

[98] Todd, M.J. (1978a), Improving the convergence of fixed point algorithms, Math. Programming Stud. 7, pp. 151-169. 
[99] TC.dd, M.J. (1978b), Fixed-point algorithms that allow restarting without an extra dimension, School of Operations Research and Industrial Engineering, Technical Report 379, Cornell University, Ithaca..

[100] Todd, M.J. (1978c), Exploiting structure in fixed-point computation, Mathematical Research Center, Technical Summary Report, University of Wisconsin, Madison.

[101] Todd, M.J. (1978d), Traversing large pieces of linearjty in algorithms that solve equations by following piecewiselinear paths, School of Operations Research and Industrial Engineering, Technical Report 390, Cornell University, Ithaca.

[102] Todd, M.J. (1978e), on the Jacobian of a function at a zero computed by a fixed point algorithm, Math. Oper. Res. $\underline{3}$, pp. 126-132.

[103] Todd, M.J. (1978f), private communication.

[104] Todd, M.J. (1980), Global and local convergence and monotonicity results for a recent variable-dimension simplicial algorithm, Numerical solution of highly nonlinear problems, w.Foster, ed., North-Holland, Amsterdam, pp. 43-69

[105] Todd, M.J. and Wright, A.H. (1979), A variable-dimension simplicial algorithm for antipodal fixed-point theorems, school of Operations Research and Industrial Engineering, Technical Report 417, Cornell University, Ithaca.

[106] Tuy, H. (1979), Pivotal methods for computing equilibrium points: Unified approach and new restart algorithm, Math. Programming 16, pp. 210-227.

[107] Von Neuman, J. (1937), Uber ein bkonomisches Gleichungssystem und eine Verallgemeinerung des Brouwerschen Fixpunktsatzes, Ergebnisse eines Mathematischen Kolloquiums $\underline{8}$, pp. 73-83.

[108] Whalley, J. (1975), Some general equilibrium analysis applied to fiscal harmonisation in the European Community, presented at the 3rd World Congress of the Econometric Society, Toronto.

[109] Whitney, H. (1957), Geometric integration theory, Princeton University Press, Princeton. 
[110] Wolsey, L.A. (1974), Convergence, simplicial paths and acceleration methods for simplicial approximation algorithms for finding a zero of a system of nonlinear equations, Center for Operations Research and Econometrics, Discussion Paper 7427, Université Catholiqụe de Louvain, Louvain-la-Neuve.

[111] Wright, A.H. (1979), The octahedral algorithm, a new simplicial fixed point algorithm, Department of Mathematics, Preprint, Western Michigan University, Kalamazoo. 


\section{OTHER TITLES IN THE SERIES MATHEMATICAL CENTRE TRACTS}

A leaflet containing an order-form and abstracts of all publications mentioned below is available at the Mathematisch Centrum, Kruislaan 413, Amsterdam 1098SJ, The Netherlands. Orders should be sent to the same address.

MCT 1 T. VAN DER WALT, Fixed and almost fixed points, 1963. ISBN 906196 0029 .

MCT 2 A.R. BLOEMENA, Sampling from a graph, 1964. ISBN 9061960037.

MCT 3 G. DE LEVE, Generalized Markovian decision processes, part I: Model and method, 1964. ISBN 9061960045 .

MCT 4 G. DE LEVE, Generalized Markovian decision processes, part II: Probabilistic background, 1964. ISBN 9061960053.

MCT 5 G. DE LEVE, H.C. TIJMS \& P.J. WEEDA, Generalized Markovian decision processes, Applications, 1970. ISBN 9061960517.

MCT 6 M.A. MAURICE, Compact ordered spaces, 1964 . ISBN 9061960061.

MCT 7 W.R. VAN ZWET, Convex transformations of random variables, 1964. ISBN $906196007 \mathrm{x}$.

MCT 8 J.A. ZONNEVELD, Automatic numerical integration, 1964. ISBN 906196 0088 .

MCT 9 P.C. BAAYEN, Universal morphisms, 1964. ISBN 9061960096.

MCT 10 E.M. DE JAGER, Applications of distributions in mathematical physics, 1964. ISBN $906196010 \mathrm{X}$.

MCT 11 A.B. PAALMAN-DE MIRANDA, Topological semigroups, 1964. ISBN 906196 0118.

MCT 12 J.A.TH.M. VAN BERCKEL, H. BRANDT CORSTIUS, R.J. MOKKEN \& A. VAN WIJNGAARDEN, Formal properties of newspaper Dutch, 1965. ISBN 9061960134 .

MCT 13 H.A. LAUWERIER, Asymptotic expansions, 1966, out of print; replaced by MCT 54 and 67 .

MCT 14 H.A. LAUWERIER, Calculus of variations in mathematical physics, 1966. ISBN 9061960207 .

MCT 15 R. DOORNBOS, Slippage tests, 1966. ISBN 9061960215.

MCT 16 J.W. DE BAKKER, Formal definition of programming Zanguages with an application to the definition of ALGOL 60, 1967. ISBN 906196 0223 .

MCT 17 R.P. VAN DE RIET, Formula manipulation in ALGOL 60, part 1, 1968. ISBN 9061960258 .

MCT 18 R.P. VAN DE RIET, Formula manipulation in ALGOL 60, part 2, 1968. ISBN $906196038 \mathrm{x}$.

MCT 19 J. VAN DER SLOT, Some properties related to compactness, 1968. ISBN 9061960266 .

MCT 20 P.J. VAN DER HOUWEN, Finite difference methods for solving partial differential equations, 1968. ISBN 9061960274 . 
MCT 21 E. WATTEL, The compactness operator in set theory and topology, 1968. ISBN 9061960282.

MCT 22 T.J. DEKKER, ALGOL 60 procedures in numerical algebra, part 1, 1968. ISBN 9061960290 .

MCT 23 T.J. DEKKER \& W. HOFFMANN, ALGOL 60 procedures in numerical algebra, part 2, 1968. ISBN 9061960304 .

MCT 24 J.W. DE BAKKER, Recursive procedures, 1971. ISBN 9061960606 .

MCT 25 E.R. PAERL, Representations of the Lorentz group and projective geometry, 1969. ISBN 9061960398.

MCT 26 EUROPEAN MEETING 1968, Selected statistical papers, part I, 1968. ISBN 9061960312 .

MCT 27 EUROPEAN MEETING 1968, Selected statistical papers, part II, 1969. ISBN 9061960401.

MCT 28 J. OOSTERHOFF, Combination of one-sided statistical tests, 1969. ISBN $906196041 \mathrm{x}$.

MCT 29 J. VERHOEFF, Error detecting decimal codes, 1969. ISBN 9061960428.

MCT 30 H. BRANDT CORSTIUS, Excercises in computational linguistics, 1970. ISBN 9061960525.

MCT 31 W. MOLENAAR, Approximations to the Poisson, binomial and hypergeometric distribution functions, 1970. ISBN 9061960533.

MCT 32 L. DE HAAN, On regular variation and its application to the weak convergence of sample extremes, 1970. ISBN 9061960541.

MCT 33 F.W. STEUTEL, Preservation of infinite divisibility under mixing and related topics, 1970. ISBN 9061960614.

MCT 34 I. JUHÁSZ, A. VERBEEK \& N.S. KROONENBERG, Cardinal functions in topology, 1971. ISBN 9061960622 .

MCT 35 M.H. VAN EMDEN, An analysis of complexity, 1971. ISBN 9061960630.

MCT 36 J. GRASMAN, On the birth of boundary Zayers, 1971. ISBN 9061960649.

MCT 37 J.W. DE BAKKER, G.A. BLAAUW, A.J.W. DUIJVESTIJN, E.W. DIJKSTRA, P.J. VAN DER HOUWEN, G.A.M. KAMSTEEG-KEMPER, F.E.J. KRUSEMAN ARETL, W.L. VAN DER POEL, J.P. SCHAAP-KRUSEMAN, M.V. WILKES \& G. ZOUTENDIJK, MC-25 Informatica Symposium, 1971. ISBN 9061960657.

MCT 38 W.A. VERLOREN VAN THEMAAT, Automatic analysis of Dutch compound words, 1971. ISBN 9061960738.

MCT 39 H. BAVINCK, Jacobi series and approximation, 1972. ISBN 9061960746.

MCT 40 H.C. TIJMS, Analysis of $(s, S)$ inventory models, 1972. ISBN 9061960754.

MCT 41 A. VERBEEK, Superextensions of topological spaces, 1972. ISBN 90 61960762.

MCT 42 W. VERVAAT, Success epochs in Bernoulli trials (with applications in number theory), 1972. ISBN 9061960770.

MCT 43 F.H. RUYMGAART, Asymptotic theory of rank tests for independence, 1973. ISBN 9061960819.

MCT 44 H. BART, Meromorphic operator valued functions, 1973. ISBN 9061960827. 
MCT 45 A.A. BALKEMA, Monotone transformations and limit lows, 1973. ISBN 9061960835 .

MCT 46 R.P. VAN DE RIET, ABC ALGOI, A portable language for formula manipulation systems, part 1: The language, 1973. ISBN 9061960843.

MCT 47 R.P. VAN DE RIET, ABC ALGOL, A portable language for formula manipulation systems, part 2: The compiler, 1973. ISBN 9061960851.

MCT 48 F.E.J. KRUSEMAN ARETZ, P.J.W. TEN HAGEN \& H.L. OUDSHOORN, An ALGOL 60 compiler in ALGOL 60, Text of the MC-compiler for the $E L-X 8,1973$. ISBN $906196086 \mathrm{x}$.

MCT 49 H. KoK, Connected orderable spaces, 1974. ISBN 9061960886.

MCT 50 A. VAN WiJNGaARDEN, B.J. MaILloux, J.E.L. PECK, C.H.A. Koster, M. SINTZOFF, C.H. LINDSEY, L.G.L.T. MEERTENS \& R.G. FISKER (Eds), Revised report on the algorithmic language ALGOL 68, 1976. ISBN 90 6196. 0894.

MCT 51 A. HORDIJK, Dynamic programming and Markov potential theory, 1974. ISBN 9061960959.

MCT 52 P.C. BAAYEN (ed.), Topological structures, 1974. ISBN 9061960967.

MCT 53 M.J. FABER, Metrizability in generalized ordered spaces, 1974. ISBN 9061960975 .

MCT 54 H.A. LAUWERIER, Asymptotic analysis, part 1, 1974. ISBN 9061960983.

MCT 55 M. HALL JR. \& J.H. VAN LINT (Eds), Combinatorics, part 1: Theory of designs, finite geometry and coding theory, 1974. ISBN 9061960991 .

MCT 56 M. HALL JR. \& J.H. VAN LINT (Eds), Combinatorics, part 2: graph theory, foundations, partitions and combinatorial geometry, 1974. ISBN 9061961009.

MCT 57 M. HALL JR. \& J.H. VAN LINT (Eds), Combinatorics, part 3: Combinatorial group theory, 1974. ISBN 9061961017.

MCT 58 W. ALBERS, Asymptotic expansions and the deficiency concept in statistics, 1975. ISBN 9061961025.

MCT 59 J.L. MIJNHEER, Somple path properties of stabie processes, 1975. ISBN 9061961076 .

MCT 60 F. GÖBEL, Queueing models involving buffers, 1975. ISBN 9061961084 .

* MCT 61 P. VAN EMDE BOAS, Abstract resource-bound classes, part 1. ISBN 9061961092 .

* MCT 62 P. VAN EMDE BOAS, Abstract resource-bound classes, part 2. ISBN 9061961106 .

MCT 63 J.W. DE BAKKER (ed.), Foundations of computer science, 1975. ISBN 9061961114 .

MCT 64 W.J. DE SCHIPPER, Symmetric closed categories, 1975. ISBN 906196 1122.

MCT 65 J. DE VRIES, Topological transformation groups 1 A categorical approach, 1975. ISBN 9061961130.

MCT 66 H.G.J. PIJLS, Locally convex algebras in spectral theory and eigenfunction expansions, 1976. ISBN 9061961149. 
* MCT 67 H.A. LAUWERIER, Asymptotic analysis, part 2. ISBN $906196119 \mathrm{x}$.

MCT 68 P.P.N. DE GROEN, Singularly perturbed differential operators of second order, 1976. ISBN 9061961203.

MCT 69 J.K. LENSTRA, Sequencing by enumerative methods, 1977. ISBN 9061961254 .

MCT 70 W.P. DE ROEVER JR., Recursive program schemes: semantics and proof theory, 1976. ISBN 9061961270 .

MCT 71 J.A.E.E. VAN NUNEN, Contracting Markov decision processes, 1976. ISBN 9061961297 .

MCT 72 J.K.M. JANSEN, Simple periodic and nonperiodic Lamé functions and their applications in the theory of conical waveguides, 1977. ISBN 9061961300 .

MCT 73 D.M.R. IEIVANT, Absoluteness of intuitionistic Zogic, 1979. ISBN $906196122 \mathrm{x}$.

MCT 74 H.J.J. TE RIELE, A theoretical and computational study of generalized aliquot sequences, 1976. ISBN 9061961319.

MCT 75 A.E. BROUWER, Treelike spaces and related connected topological spaces, 1977. ISBN 9061961327.

MCT 76 M. REM, Associons and the closure statement, 1976. ISBN 9061961351.

MCT 77 W.C.M. KALLENBERG, Asymptotic optimality of likelihood ratio tests in exponential families, 1977 ISBN 9061961343.

MCT 78 E. DE JONGE, A.C.M. VAN ROOIJ, Introduction to Riesz spaces, 1977. ISBN 9061961335.

MCT 79 M.C.A. VAN ZUIJLEN, Empirical distributions and rankstatistics, 1977. ISBN 9061961459.

MCT 80 P.W. HEMKER, A numerical study of stiff two-point boundary problems, 1977. ISBN 9061961467 .

MCT 81 K.R. APT \& J.W. DE BAKKER (Eds), Foundations of computer science II, part 1, 1976. ISBN 9061961408.

MCT 82 K.R. APT \& J.W. DE BAKKER (Eds), Foundations of computer science II, part 2, 1976. ISBN 9061961416.

MCT 83 L.S. VAN BENTEM JUTTING, Checking Landau's "Gmundagen" in the AUTOMATH system, 1979 ISBN 9061961475.

MCT 84 H.L.L. BUSARD, The translation of the elements of Euclid from the Arabic into Latin by Hermann of Carinthia (?) books vii-xii, 1977. ISBN 9061961483

MCT 85 J. VAN MILL, Supercompactness and WaZZman spaces, 1977. ISBN 9061961513.

MCT 86 S.G. VAN DER MEULEN \& M. VELDHORST, TOMPix I, 1978. ISBN 9061961521 .

* MCT 87 S.G. VAN DER MEULEN \& M. VELDHORST, TOMrix II, ISBN $906196153 \mathrm{x}$.

MCT 88 A. SCHRIJVER, Matroids and Zinking systems, 1977. ISBN 9061961548 . 
MCT 89 J.W. DE ROEVER, Complex Fourier transformation and analytic functionals with unbounded carriers, 1978. ISBN 9061961556.

* MCT 90 L.P.J. GROENEWEGEN, Characterization of optimal strategies in dynamic games, . ISBN 9061961564.

MCT 91 J.M. GEYSEL, Transcendence in fields of positive characteristic, 1979. ISBN 9061961572 .

MCT 92 P.J. WEEDA, Finite generalized Markov programing,1979. ISBN 9061961580.

MCT 93 H.C. TIJMS (ed.) \& J. WESSELS (ed.), Markor decision theory, 1977. ISBN 9061961602 .

MCT 94 A. BIJLSMA, Simultaneous approximations in transcendental number theory, 1978 . ISBN 9061961629.

MCT 95 K.M. VAN HEE, Bayesian control of Markov chains, 1978 . ISBN 9061961637.

MCT 96 P.M.B. VITÁNYI, Lindenmayer systems: structure, languages, and growth functions, 1980. ISBN 9061961645 .

* MCT 97 A. FEDERGRUEN, Markovian control problems; functional equations and algorithms, . ISBN 9061961653.

MCT 98 R. GEEL, Singular perturbations of hyperbolic type, 1978. ISBN 9061961661

MCT 99 J.K. LENSTRA, A.H.G. RINNOOY KAN \& P. VAN EMDE BOAS, Interfaces between computer science and operations research, 1978. ISBN $906196170 \mathrm{x}$.

MCT 100 P.C. BAAYEN, D. VAN DULST \& J. OOSTERHOFF (Eds), Proceedings bicentennial congress of the Wiskundig Genootschap, part 1,1979. ISBN 9061961688.

MCT 101 P.C. BAAYEN, D. VAN DULST \& J. OOSTERHOFF (Eds), Proceedings bicentennial congress of the Wiskundig Genootschop, part 2,1979. ISBN 9091961696.

MCT 102 D. VAN DULST, Reflexive and superreflexive Banach spaces, 1978. ISBN 9061961718.

MCT $103 \mathrm{~K}$. VAN HARN, Classifying infinitely divisible distributions by functional equations, 1978 . ISBN 9061961726.

MCT 104 J.M. VAN WOUWE, Go-spaces and generalizations of metrizability,1979. ISBN 9061961734.

* MCT 105 R. HELmers, Edgeworth expansions for linear combinations of order statistics, . ISBN 9061961742.

MCT 106 A. SCHRIJVER (Ed.), Packing and covering in combinatorics, 1979. ISBN 9061961807.

MCT 107 C. DEN HEIJER, The numerical solution of nonlinear operator equations by imbedding methods, 1979. ISBN 9061961750 .

MCT 108 J.W. DE BAKKER \& J. VAN LEEUWEN (Eds), Foundations of computer science III, part 1, 1979. ISBN 9061961769. 
MCT 109 J.W. DE BAKKER \& J. VAN LEEUWEN (Eds), Foundations of computer science III, part 2, 1979. ISBN 9061961777.

MCT 110 J.C. VAN VLIET, ALGOL 68 transput, part I: Historical Review and Discussion of the Implementation Model, 1979. ISBN 9061961785.

MCT 111 J.C. VAN VLIET, ALGOL 68 transput, part II: An implementation model, 1979. ISBN 9061961793.

MCT 112 H.C.P. BERBEE, Random walks with stationary increments and Renewal theory, 1979. ISBN 906196.1823.

MCT 113 T.A.B. SNIJDERS, Asymptotic optimality theory for testing problems with restricted alternatives, 1979. ISBN 9061961831 .

MCT 114 A.J.E.M. JANSSEN, Application of the Wigner distribution to harmonic analysis of generalized stochastic processes, 1979. ISBN $906196184 \mathrm{x}$.

MCT 115 P.C. BAAYEN \& J. VAN MILL (Eds), Topological Structures II, part 1, 1979. ISBN 9061961855 .

MCT 116 P.C. BAAYEN \& J. VAN MILL (Eds), Topological Structures II, part 2, 1979. ISBN 9061961866 .

MCT 117 P.J.M. KALLENBERG, Branching processes with continuous state space, 1979. ISBN 9061961882 .

MCT 118 P. GROENEBOOM, Large deviations and Asymptotic efficiencies, 1980. ISBN 9061961904 .

MCT 119 F. PETERS, Sparse matrices and substmuctures, 1980. ISBN 906196 1920 .

MCT 120 W.P.M. DE RUYTER, On the Asymptotic Analysis of Large Scale Ocean Circulation, 1980. ISBN 9061961929.

MCT $121 \mathrm{~W}_{0} \mathrm{H}$. HAEMERS, Eigenvalue techniques in design and graph theory, 1980. ISBN 9061961947.

MCT 122 J.C.P. BUS, Numerical solution of systems of nonlinear equations, 1980. ISBN 9061961955.

MCT 123 I. YUHÁsZ, Cardinal functions intopology - ten years Zater, 1980. ISBN 9061961963.

MCT 124 R.D. GILL, Censoring and Stochastic Integrals, 1980. ISBN 906196 1971.

MCT 125 R. EISING, 2-D Systems, an aZgebaric approach, 1980. ISBN 906196 $198 \mathrm{X}$.

MCT 126 G. VAN DER HOEK, Reduction methods in nonlinear programming, 1980. ISBN 9061961998 .

MCT 127 J.W. KLOP, Combinatory reduction systems, 1980. ISBN 9061962005.

MCT 128 A.A.J. TALMAN, Variable dimension fixed point algorithms and triangulations, 1980. ISBN 9061962013.

MCT 129 G. van der LAAN, Simplicial fixed point algorithms, 1980. ISBN 9061962021 .

MCT 130 P.J.W. TEN HAGEN a.o., ILP Intermediate Zanguage for pictures, 1980. ISBN 9061962048. 
MCT 131 R.J.R. BACK, Correctness preserving program refinements: Proof theory and applications, 1980. ISBN 9061962072 .

MCT 132 H.M. Mulder, The interval function of a graph, 1980.

ISBN 9061962080 . 
